\title{
WestVirginiaUniversity
}

THE RESEARCH REPOSITORY @ WVU

Graduate Theses, Dissertations, and Problem Reports

2020

\section{Terrain Aware Traverse Planning for Mars Rovers}

Gabrielle Hedrick

glc0006@mix.wvu.edu

Follow this and additional works at: https://researchrepository.wvu.edu/etd

Part of the Aerospace Engineering Commons

\author{
Recommended Citation \\ Hedrick, Gabrielle, "Terrain Aware Traverse Planning for Mars Rovers" (2020). Graduate Theses, \\ Dissertations, and Problem Reports. 7956. \\ https://researchrepository.wvu.edu/etd/7956
}

This Dissertation is protected by copyright and/or related rights. It has been brought to you by the The Research Repository @ WVU with permission from the rights-holder(s). You are free to use this Dissertation in any way that is permitted by the copyright and related rights legislation that applies to your use. For other uses you must obtain permission from the rights-holder(s) directly, unless additional rights are indicated by a Creative Commons license in the record and/ or on the work itself. This Dissertation has been accepted for inclusion in WVU Graduate Theses, Dissertations, and Problem Reports collection by an authorized administrator of The Research Repository @ WVU.

For more information, please contact researchrepository@mail.wvu.edu. 


\title{
TERRAIN AWARE TRAVERSE PLANNING FOR MARS ROVERS
}

\author{
Gabrielle Hedrick
}

Dissertation submitted to the Statler College of Engineering and Mineral Resources at West Virginia University

in partial fulfillment of the requirements

for the degree of

\author{
Doctor of Philosophy \\ in \\ Aerospace Engineering \\ Yu Gu, Ph.D., Committee Chairperson \\ Jason Gross, Ph.D. \\ Marcello Napolitano, Ph.D. \\ Powsiri Klinkhachorn, Ph.D. \\ Timothy Warner, Ph.D.
}

Mechanical and Aerospace Engineering

Morgantown, West Virginia

2020

Keywords: Mars, terrain, terramechanics, path planning, fetch rover

Copyright 2020, Gabrielle Hedrick 


\section{Abstract \\ TERRAIN AWARE TRAVERSE PLANNING FOR MARS ROVERS}

\section{by Gabrielle Hedrick}

NASA is proposing a Mars Sample Return mission, to be completed within one Martian year, that will require enhanced autonomy to perform its duties faster, safer, and more efficiently. With its main purpose being to retrieve samples possibly tens of kilometers away, it will need to drive beyond line-of-sight to get to its target more quickly than any rovers before. This research proposes a new methodology to support a sample return mission and is divided into three components: map preparation (map of traversability, i.e., ability of a terrain to sustain the traversal of a vehicle), path planning (pre-planning and replanning), and terrain analysis. The first component aims at creating a better knowledge of terrain traversability to support planning, by predicting rover slip and drive speed along the traverse using orbital data. By overlapping slope, rock abundance and terrain types at the same location, the expected drive velocity is obtained. By combining slope and thermal data, additional information about the experienced slip is derived, indicating whether it will be low (less than $30 \%$ ) or medium to high (more than 30\%). The second component involves planning the traverse for one Martian day (or sol) at a time, based on the map of expected drive speed. This research proposes to plan, offline, several paths traversable in one sol. Once online, the rover chooses the fastest option (the path cost being calculated using the distance divided by the expected velocity). During its drive, the rover monitors the terrain via analysis of its experienced wheel slip and actual speed. This information is then passed along the different pre-planned paths over a given distance (e.g., $25 \mathrm{~m}$ ) and the map of traversability is locally updated given this new knowledge. When an update occurs, the rover calculates the new time of arrival of the various paths and replans its route if necessary. When tested in a simulation study on maps of the Columbia Hills, Mars, the rover successfully updates the map given new information drawn from a modified map used as ground truth for simulation purposes and replans its traverse when needed. The third component describes a method to assess the soil in-situ in case of dangerous terrain detected during the map update, or if the monitoring is not enough to confirm the traversability predicted by the map. The rover would deploy a shear vane instrument to compute intrinsic terrain parameters, information then propagated ahead of the rover to update the map and replan if necessary. Experiments in a laboratory setting as well as in the field showed promising results, the mounted shear vane giving values close to the expected terrain parameters of the tested soils. 


\section{DEDICATION}
"Easy will not get you to Mars."
- Jake H.

I wish to dedicate this work to my son Killian, the catalyst that led me to finally graduate. It has been a long journey with many bumps (virtual and real) on the road, a journey that saw me start a Ph.D., leave for the French Air Force Reserves, come back, move halfway across the country, start over my Ph.D. in a much more suitable field for me, become a first responder and start a family. Killian's birth gave me the boost I needed to finish my degree, and after nine years, it finally happened. It has been a long and difficult journey, but as we all know, "easy" is not what will get me to space. 


\section{ACKNOWLEDGMENTS}

I wish to express my gratitude to my advisor, Dr. Yu Gu, for his guidance throughout the years, his patience, his Perseverance with me and for always keeping me in the Spirit of graduate school. I am also grateful for the Opportunity to work again on the Mars rovers with JPL. I am thankful for my committee members as well for their help on my research, and a special thank you to Dr. Warner for giving me unlimited access to his laboratory and software that I used extensively during my four years at WVU. I am grateful for Dr.Ono's contribution at JPL, his help and guidance on some aspect of my dissertation, and his welcoming into his team was also greatly appreciated. I would like to finally acknowledge the other IRL students for their collaboration on this research, and more specifically Nicholas Ohi and Dylan Covell.

I wish to acknowledge B. Statler and NASA EPSCoR Research Cooperative Agreement WV-80NSSC17M0053 for the financial support throughout my Ph.D., that allowed me to be successful in all the steps that led to graduation. Thanks to the Benjamin M. Statler fellowship and NASA funding, I was able to start a family while advancing in my studies, which has been more than beneficial in every aspect of my student life. I thank West Virginia University for its financial support regarding child care, which helped tremendously in finishing my degree.

And last but not least, I want to mention all the people who have been there for me one way or another, starting with my husband Jesse. He made sure I would not give up and he pushed me every single day, for which I am thankful. And, of course, he gave me Killian, the cutest, most incredible human being and a local celebrity already way too smart for his own good.

And everyone else who has been there during this incredibly challenging time: my fire department friends who helped getting me ready for motherhood in the midst of graduate school, and my midwife Kate; my frisbee peeps, their children and the people at Morgantown Campground who helped tremendously with Killian while finishing up this document; my EMS family, including Gary who makes Medcom calls easier and the "dream team" Anna and Bobby, who got me through FTO, somehow; Lauren at ME Morgantown for relieving the stress I was under.

There is actually one more paragraph that has a rightful place here: I want to acknowledge Kindle Unlimited $^{\circledR}$ that provided me with a way to relax amidst 2020 craziness and my son becoming a toddler; West Virginia that gave me a husband, a son and a doctorate; and Mars, who's always been there for me for the past nine years! 


\section{Contents}

List of Figures $\quad$ x

List of Tables $\quad$ xiv

1 Goal and High-level Statement 1

2 Introduction $\quad 2$

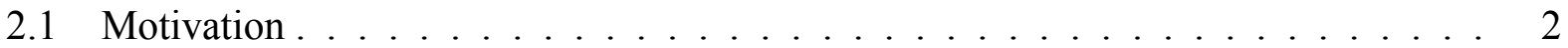

2.1 .1 Missions to Mars . . . . . . . . . . . . . . . . . . 2

2.1.2 Mars Sample Return (MSR) and the proposed fetch rover . . . . . . . . . . 2

2.1.3 More autonomy needed for MSR and future space missions . . . . . . . . 3

2.1.4 The importance of understanding the terrain for planning . . . . . . . . . 4

2.2 Terrain related background information available to the fast traverse rover . . . . 5

2.2.1 Processed orbital analysis to support traversability assessment . . . . . . 5

2.2.1.1 Cumulative Fractional Area (CFA) . . . . . . . . . . . 5

2.2.1.2 Terrain classification software . . . . . . . . . . . 6

2.2.1.3 Tool to determine traversability on Mars . . . . . . . . . . . . 9

2.2.2 Terrain parameters to support traversability assessment . . . . . . . . . 10

2.2.2.1 Terrain properties indicative of traversability . . . . . . . . . 10

2.2.2.2 Measurement of various soil parameters . . . . . . . . . . 11

3 Problem Statement $r$

3.1 Objectives of the research . . . . . . . . . . . . . . . . . . 14

3.2 Information available to support this research $\ldots \ldots \ldots \ldots \ldots$

3.3 Constraints related to Mars missions . . . . . . . . . . . . . . . . . 15

3.4 Assumptions applicable to this research . . . . . . . . . . . . . 16

3.5 Challenges of a fast traversing sample return rover . . . . . . . . . . 16

3.6 Contributions of this work . . . . . . . . . . . . . . . 17

4 Literature Review $\quad 18$

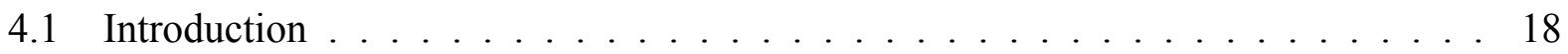

4.2 Path planning . . . . . . . . . . . . . . . . . . 18

4.2 .1 Overview and definitions . . . . . . . . . . . . . . 18

4.2.2 Sampling-based algorithms . . . . . . . . . . . . . . . . 18

4.2.2.1 Probabilistic Road Map (PRM) . . . . . . . . . . . . . . . . . 19

4.2.2.2 Rapidly exploring Random Tree (RRT) . . . . . . . . . . . . . . 19

4.2 .3 Search algorithms . . . . . . . . . . . . . . . 21 
4.2 .4 Planning with a budget constraint $\ldots \ldots \ldots \ldots \ldots$

4.2 .5 Planning under uncertainties . . . . . . . . . . . . . . . . . . 24

4.2.5.1 Markov Random Field (MRF) and belief propagation . . . . . 24

4.2.5.2 Other approaches . . . . . . . . . . . . . . 26

4.3 Terramechanics . . . . . . . . . . . . . . . . . . . . . . . . 28

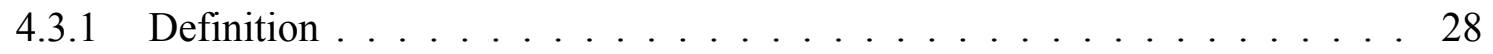

4.3 .2 Historical background . . . . . . . . . . . . . . . . 28

4.3.3 Terramechanics equations for a driven wheel . . . . . . . . . . . . . 29

4.3 .4 Rover problem . . . . . . . . . . . . . . . . . . . . . . . . 29

4.3.5 Deformable wheels . . . . . . . . . . . . . . . . . 30

4.4 Exploration rovers: terramechanics and terrain information integrated into path planning under terrain uncertainty . . . . . . . . . . . . 30

4.4.1 Terramechanics and path planning . . . . . . . . . . . . 31

4.4 .2 Path planning under terrain uncertainty . . . . . . . . . . . . . 32

4.4.3 Geotechnical properties of soils that can support planning . . . . . . 33

5 Technical Approach $\quad 35$

5.1 Introduction . . . . . . . . . . . . . . . . . 35

5.2 Orbital traversability map: slip and velocity prediction $\ldots \ldots \ldots 35$

5.2 .1 Problem statement . . . . . . . . . . . . . . . . . . . 35

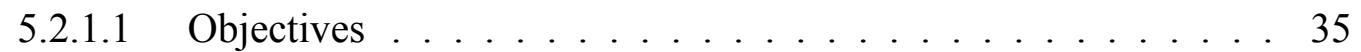

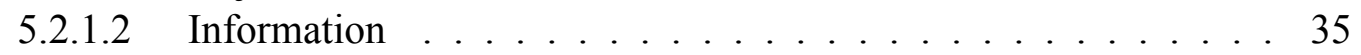

5.2 .1 .3 Constraints . . . . . . . . . . . . . . . . 36

5.2 .1 .4 Assumptions . . . . . . . . . . . . . . . 36

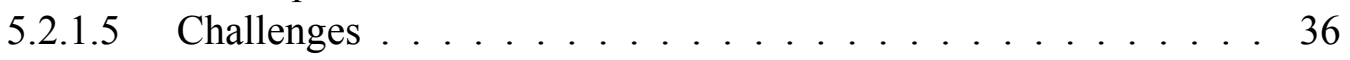

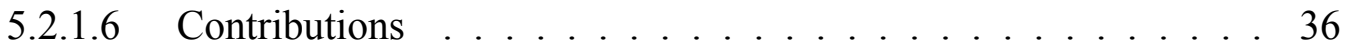

5.2 .2 Orbital data sets . . . . . . . . . . . . . . . . . . . 36

5.2.2.1 Terrain types and traversability classes . . . . . . . . . 36

5.2.2.2 Cumulative Fractional Area (CFA) . . . . . . . . . . . . . 37

5.2 .2 .3 Slope . . . . . . . . . . . . . . . . . . 37

5.2.2.4 Thermal inertia . . . . . . . . . . . . . . . . . 38

5.2 .3 Velocity prediction . . . . . . . . . . . . . . . 40

5.2.3.1 Velocity categories . . . . . . . . . . . . . . . . 40

5.2.3.2 Map of velocity categories _. . . . . . . . . . . . 40

5.2 .4 Slip prediction . . . . . . . . . . . . . . . . . . . 41

5.2.4.1 Principal Component Analysis (PCA) . . . . . . . . . . . 41

5.2.4.2 Classifier training to predict slip . . . . . . . . . . 41

5.2.4.2.1 Data points available . . . . . . . . . . 41

5.2 .4 .2 .2 Data processing . . . . . . . . . . . . 42

5.2.4.2.3 Classifier training . . . . . . . . . . . . . 43

5.3 Terrain-aware path planning $\ldots \ldots \ldots \ldots . \ldots \ldots 4$

$5.3 .1 \quad$ Problem statement . . . . . . . . . . . . . . . . . . 44 


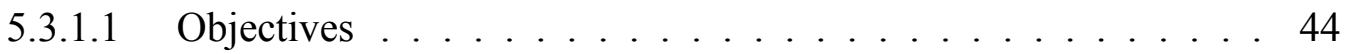

5.3 .1 .2 Information . . . . . . . . . . . . . . . 45

5.3.1.3 Constraints . . . . . . . . . . . . . . . . . 45

5.3 .1 .4 Assumptions . . . . . . . . . . . . . . . . . 45

5.3 .1 .5 Challenges . . . . . . . . . . . . . . . 46 46

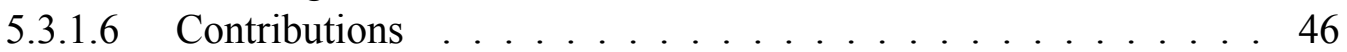

5.3 .2 Approach . . . . . . . . . . . . . . . . . 4 46

5.3.2.1 Path planning approach . . . . . . . . . . . . . 46

5.3.2.2 Terrain monitoring: noisy measurements . . . . . . . . . . 47

5.3 .3 Map and map update . . . . . . . . . . . . . . . . . . 50

5.3 .3 .1 Belief map . . . . . . . . . . . . . . . 50

5.3.3.1.1 Class confusion matrix . . . . . . . . . 50

5.3.3.1.2 Probability Mass Functions (PMFs) of velocity cate-

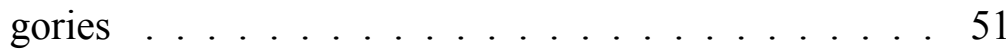

5.3.3.1.3 Belief map of velocity category . . . . . . . . . . 52

5.3.3.2 Local Map Update . . . . . . . . . . . . . . . . . . . . . 53

5.3.3.2.1 Edge potential matrices . . . . . . . . . . 53

5.3.3.2.2 Belief propagation framework . . . . . . . . . 53

5.3 .4 Replanning . . . . . . . . . . . . . . . . . . . . . . 54

5.4 Parameters and instrumentation to assess terrains $\ldots \ldots \ldots \ldots$

5.4 .1 Problem statement . . . . . . . . . . . . . . . . . . . . 54

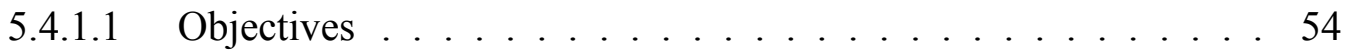

5.4 .1 .2 Information . . . . . . . . . . . . . . . 54

5.4 .1 .3 Constraints . . . . . . . . . . . . . . . . . 54

5.4 .1 .4 Assumptions . . . . . . . . . . . . . . 55

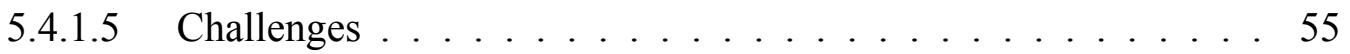

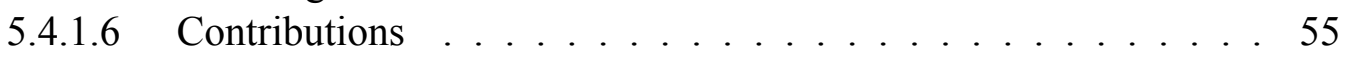

5.4 .2 Instrument to assess terrain properties . . . . . . . . . . . 55

5.4.2.1 Choice of instrumentation . . . . . . . . . . . . . 55

5.4.2.2 Hand held pocket shear vane . . . . . . . . . . . 56

5.4 .2 .3 Mounted shear vane . . . . . . . . . . . . . . . 57

5.4 .3 Instrument deployment $\ldots \ldots \ldots \ldots \ldots \ldots$

6 Results 61

6.1 Map of traversability . . . . . . . . . . . . . . . . . . . 61

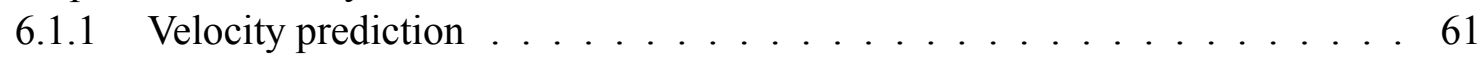

6.1 .2 Slip prediction . . . . . . . . . . . . . . . . . . 62

6.1 .2 .1 Classifier training . . . . . . . . . . . . . . 62

6.1.2.2 Validation on new data . . . . . . . . . . . . . 63

6.2 Terrain-aware path planning . . . . . . . . . . . . . . . . . . . 64

6.2 .1 Measurements and cost calculation . . . . . . . . . . . 65

6.2 .2 Trial one . . . . . . . . . . . . . . . . . 66 
6.2 .3 Trial two . . . . . . . . . . . . . . . . . . 68

6.2 .4 Trial three . . . . . . . . . . . . . . . . . . . . . . . . . . 69

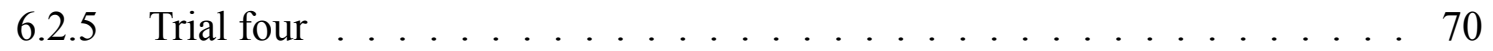

6.2 .6 Trial five . . . . . . . . . . . . . . . . . 72

6.2.7 Summary of trials and discussion $\ldots \ldots \ldots$. . . . . . . . . . 72

6.3 Instrumentation for in-situ soil analysis . . . . . . . . . . . . . . 73

6.3.1 Hand-held pocket shear vane . . . . . . . . . . . . . . . . . . . 73

6.3.1.1 Testing settings . . . . . . . . . . . . . 73

6.3.1.2 Results of tests in laboratory setting . . . . . . . . . . . . 74

6.3.1.2.1 Clay of low plasticity (USCS CL) . . . . . . . . 74

6.3.1.2.2 Silt loam, compacted (USCS ML, OL, MH, OH) . . . 75

6.3.1.2.3 Fine sand (USCS SW, SP) _ . . . . . . . . . 75

6.3.1.3 Results of tests in field setting . . . . . . . . . . . 76

6.3.1.3.1 Sand (USCS SW, SP) . . . . . . . . . . . . 76

6.3.1.3.2 Silt loam, saturated (USCS ML, OL, MH, OH) . . . 76

6.3.1.4 Interpretation of results . . . . . . . . . . . . . 76

6.3 .2 Mounted shear vane . . . . . . . . . . . . . . . . . . 77

6.3.2.1 Testing settings _ . . . . . . . . . . . . . . 77

6.3.2.2 Results of tests in laboratory setting . . . . . . . . . . . . 78

6.3.2.3 Results of tests in field setting . . . . . . . . . . . . . . . 79

6.3.2.4 Interpretation of results . . . . . . . . . . . . . 79

6.3.3 Estimate of ultimate bearing capacity for various soils . . . . . . . . . 79

6.3.3.1 Hand-held shear vane: derived Mohr circles . . . . . . . . . 80

6.3.3.2 Mounted shear vane: derived Mohr circles . . . . . . . . . . . 81

6.3 .3 .3 Overall results . . . . . . . . . . . . . . . 81

6.3 .4 Conclusion . . . . . . . . . . . . . . . . . . . . . 82

6.4 Summary of results $\ldots \ldots \ldots \ldots$

7 Discussion and Conclusion $\quad \mathbf{8 4}$

7.1 Discussion and future work . . . . . . . . . . . . . . . . . . . 84

7.1 .1 Map of traversability information . . . . . . . . . . . 84

7.1.2 Terrain-aware path planning . . . . . . . . . . . . . . 84

7.1.3 Instrument deployment in support of planning . . . . . . . . . . . 85

7.1.4 Terrain-aware traverse planning as a framework to support a sample return

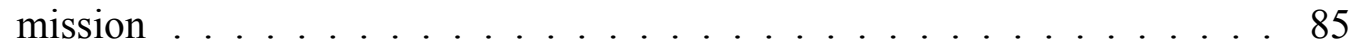

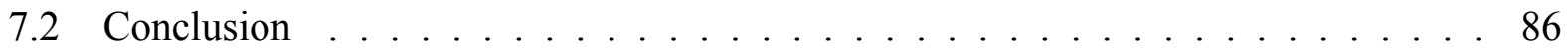

$\begin{array}{lr}\text { Bibliography } & \mathbf{8 8}\end{array}$

$\begin{array}{llr}\text { Appendix A Terramechanics Equations } & 102\end{array}$

A.1 Pressure and shear stress . . . . . . . . . . . . . . . . . . . . 103

A.2 Soil deformation under a driven wheel . . . . . . . . . . . . . . 106

A.3 Compaction resistance . . . . . . . . . . . . . . . 110 
Appendix B Probability Mass Functions of velocity categories 


\section{List of Figures}

2.1 Communication between mission control on Earth and the rovers on Mars via satellites such as Mars Reconnaissance Orbiter (MRO) and the Deep Space Network

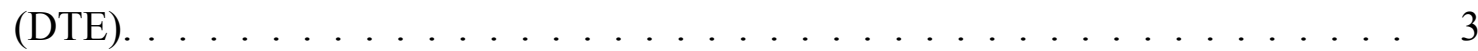

2.2 Best fit ellipse and cylinder to estimate shadow and rock sizes [Golombek et al., 2012, Golombek et al., 2008]. Photo credit:NASA . . . . . . . . . . . . . . 5

2.3 Traversability classes output from MTTT [Ono et al., 2018]. Credit: Hedrick et

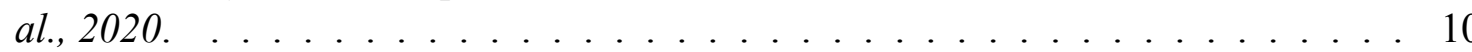

2.4 Mohr circles from principal stresses $\sigma_{1}$ and $\sigma_{3}$ leading to the Mohr-Coulomb envelope and failure point. . . . . . . . . . . . . . . . 11

2.5 Digital Cone Penetrometer by Humbolt. . . . . . . . . . . . . . . . . . . . . . . . 12

2.6 Field shear vane with extension rods and different sizes of blades. Credit: Humbolt. 12

3.1 Approach to path planning given terrain information. . . . . . . . . . . 15

4.1 Illustration of the implementation of a PRM algorithm: (a) C-space is sampled randomly, and (b) samples are checked for collision. (c) nodes are connected to their nearest neighbors and (d) edges are checked for collision. (e) the path from start to goal is found using the remaining edges. . . . . . . . . . . . . . . . 20

4.2 Illustration of RRT: (a) random sampling; (b) selection of nearest neighbor, and if in free space, addition of a new node at step-size distance from nearest neighbor with connecting edge; (c) random sampling; (d) Selection of nearest neighbor and collision detection; (e) collision detection; (f) random sampling, selection of nearest neighbor and addition of a node step-size away in the direction of random sample. . . . . . . . . . . . . . . . . . 22

4.3 (a) Directed graph and (b) equivalent factor graph. . . . . . . . . . . . 26

4.4 Message passing on a couple of nodes. . . . . . . . . . . . . 26

4.5 Curiosity wheel showing holes after traversing rough terrain with sharp rocks. Credit: NASA JPL. . . . . . . . . . . . . . . . . . . . . . . . . . . . . . . . . . . 28

4.6 Drawbar pull, thrust and resistances for a vehicle driving uphill. Credit: G.Coutrot. 30

4.7 Principle of a spectrometer as a guide to exploration: scientists create a hypothetical geology map that can be updated as the rover gains more scientific information about the terrain [Candela et al., 2017] . . . . . . . . . . . . . . . 33

5.1 Manually interpreted map of traversability classes [Hedrick et al., 2020] (comprised of several terrain types each as shown in Ono et al. (2018)) . . . . . . . . . . . . 37 
5.2 Manually interpreted map of CFA ranges: 1 - low rock abundance $(<7 \%), 2$ medium rock abundance (between $7 \%$ and $15 \%$ ) and 3 - high rock abundance ( $>$ 15\%) [Hedrick et al., 2020]. . . . . . . . . . . . . . . . . . . 38

5.3 Digital Terrain Model (DTM) of the Columbia Hills. Credit:NASA/Washington University in St. Louis. . . . . . . . . . . . . . . . . . . . . . . . . . . . . . . 39

5.4 Thermal inertia map over Gusev Crater. Values are in Thermal Inertia Unit. Credit: USGS . . . . . . . . . . . . . . . . . . . . .

5.5 Velocity categories [Hedrick et al., 2020]: A - $64.8 \mathrm{~m} / \mathrm{hr} ; \mathrm{B}-52.5 \mathrm{~m} / \mathrm{hr} ; \mathrm{C}-24.2-$ $48.5 \mathrm{~m} / \mathrm{h}$; D - 10.9-40.8 m/hr; E - $10.9 \mathrm{~m} / \mathrm{hr}$; and F - untraversable $(>15 \%$ CFA or $>25^{\circ}$ slope, not shown).

5.6 Biplot showing the contribution of each variable to the first two PCs that explain $75 \%$ of the data. TI: thermal inertia; TT: terrain types; CFA: cumulative fractional

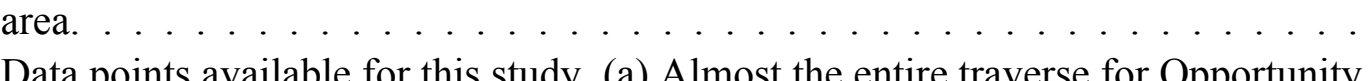

5.7 Data points available for this study. (a) Almost the entire traverse for Opportunity provides slip checks but the middle segment does not include processed DTM coverage. (b) Most of Spirit traverse is not available (no VO performed), but the entire traverse has processed DTM coverage. (c) Legend indicating the traverse with no slip checks, the slip checks that have DTM coverage and the slip checks that do not. 43

5.8 Diagram showing the one-time offline planning and the online loop comprised of update and replanning Credit: Hedrick et al., 2020 . . . . . . . . . . . . . . . 47

5.9 Slip vs. velocity with marginal distribution over category A (encompasses terrain types 1 and 2). . . . . . . . . . . . . . . . . . . . . . . . . . . . . . . 49

5.10 PDFs of (a) slip and (b) speed for each velocity category. . . . . . . . . . . . 50

5.11 Pocket shear vane with standard blades (left) and two other sizes of blades. Credit: Gilson inc. . . . . . . . . . . . . . . . . . . . . . . . . 55

5.12 Mohr-Coulomb envelope and failure point shown for a vertical load (rover weight) on a terrain. The confining pressure is negligible [Sullivan et al., 2011]. When reaching failure, $\sigma_{1}$ represents the maximum load the terrain can sustain, which is by definition the ultimate bearing capacity. . . . . . . . . . . . . . 56

5.13 Modified pocket shear vane tester including: a 3D printed cast with weight slots; calibration weights. . . . . . . . . . . . . . . . 57

5.14 Automated shear vane test prototype. Credit: D. Covell. . . . . . . . . . . . 58

5.15 Mounted shear vane device inside Fast Traverse Rover (FTR). Credit: D. Covell. . 59

6.1 (a) HiRISE image (PSP 0017771650 RED A 01) with Spirit's traverse. Spirit's traverse is shown in blue. (b) traversability classes $(1,2,3,5)$. (c) CFA map: 1, low CFA $(<7 \%)$, 2, medium CFA (between $7 \%$ and $15 \%)$ and 3 , high CFA $(>15 \%)$. (d) DTM with subset maps numbered according to each trial performed (see Table 6.1 in section 5.3). The maps are $2915 \mathrm{~m}$ by $3540 \mathrm{~m}$ and the subset maps measure $301 m$ by $401 \mathrm{~m}$. . . . . . . . . . . . . . . . . . . . . . . . .

6.2 Example map of velocity categories (subset of the Columbia Hills area shown in Fig.6.1). This example is labeled 1 on Fig.6.1d. . . . . . . . . . . . . . . 62 
6.3 Confusion matrices showing the performance of the trained model on data set B2 for (a) thermal inertia and slope as predictors and (b) slope only as predictor. . . . . 63

6.4 Confusion matrices on test data sets A and B1 for (a) model with 2 predictors and (b) model with 1 predictor. Cells in green shows the correct prediction whereas red cells shows the errors from the classifier. The overall performance is displayed in

6.5 Comparison of (a) expected velocity categories at the Columbia Hills and (b) actual velocity categories based on further analysis of HiRISE images at this specific location. The image shows that there could be a rougher terrain near the craters, reflected in the "truth" map in (b). . . . . . . . . . . . . . . . . . 65

6.6 Inital map of velocity categories A to F, with 37 paths. Credit: Hedrick et al., 2020. 66

6.7 Trial 1: actual path on (a) updated map and (b) "truth" map. One exact measurement is performed due to potential dangerous terrain ahead. One replanning event occurs in the traverse, saving about seven minutes.

6.8 Belief propagation tree passing the message of a newly detected category $\mathrm{C}$, which is more challenging than $\mathrm{A}$. The message passing leads to the rover making the decision to choose a faster path south of its original traverse, that would cross less of the more difficult terrain. . . . . . . . . . . . . . . . . . . . 68

6.9 Belief propagation tree with noisy measurement giving category A (even though, in truth, it is located on a patch of category $\mathrm{C}$ ) but detecting accurately category $\mathrm{C}$ ahead.

6.10 Trial 2: actual path (equivalent to initial path) on (a) updated map and (b) "truth" map. There is no replanning necessary, even after an exact measurement is performed due to potential dangerous terrain detected ahead. . . . . . . . . . . . .

6.11 Trial 3: actual path on (a) updated map and (b) "truth" map. One replanning event is performed to avoid more difficult terrain. . . . . . . . . . . . . 70

6.12 Trial 4: actual path on (a) updated map and (b) "truth" map . . . . . . . . . . . . . 71

6.13 Trial 5: actual path on (a) updated map and (b) "truth" map with three replanning events. The replanning leads to following a safer terrain $(\mathrm{C}$ instead of $\mathrm{D}) . \quad$. . . . . 72

6.14 Clay of low plasticity. Results give $c=85 \mathrm{kPa}$ and $\phi=33^{\circ}$. Some data points overlap with each other. Photo credit: agrclassroomstore.com. . . . . . . . . . . . 74

6.15 Compacted silt loam. Results give $c=90 \mathrm{kPa}$ and $\phi=29^{\circ}$. Some data points overlap with each other. Photo credit: agrclassroomstore.com. . . . . . . . . 75

6.16 Tests in fine grain sand material of known parameters. Photo credit: agrclassroomstore.com. . . . . . . . . . . . . . . . 76

6.17 Test of the pocket shear vane in cohesionless sand under dry conditions. Photo credit: G. Hedrick. . . . . . . . . . . . . . . . . . . . . 77

6.18 Hand-held instrument tested in Silt Loam, saturated (in-situ). Photo credit: $G$. Hedrick . . . . . . . . . . . . . . . . . . . . 77

6.19 Mounted instrument tested in Clay of low plasticity, compacted. Results give $c=$ $90 \mathrm{kPa}$ and $\phi=34^{\circ}$. Some data points overlap with each other, while others were eliminated due to being unrealistics. Photo credit: agrclassroomstore.com . . . . . 79 
6.20 Mounted instrument tested in Silt loam, saturated (in-situ). Results give $c=20 \mathrm{kPa}$ and $\phi=24^{\circ}$. Some data points overlap with each other, while others were eliminated. Photo credit: G. Hedrick. . . . . . . . . . . . . . . . . . . . . 80

6.21 Estimation of the ultimate bearing capacity for laboratory tests. Compacted clay shows $q_{u l t}=310 \mathrm{kPa}$ and compacted silt loam can hold up to $q_{u l t}=308 \mathrm{kPa} . \ldots 81$

6.22 Mohr circle at failure and Mohr-Coulomb envelope for in-situ silt loam, saturated. The result show that saturated silt loam can bear $q_{u l t}=66 \mathrm{kPa}$ at most. . . . . . 81

6.23 Estimation of the ultimate bearing capacity for laboratory and field tests. Compacted clay shows $q_{u l t}=330 \mathrm{kPa}$ and saturated silt loam can hold up to $q_{u l t}=60 \mathrm{kPa} .82$

7.1 Framework supporting more autonomy for a Mars Sample Return mission. . . . . . 87

A.1 Driven wheel with velocities and forces applied on a given point $K$ on the circumference of the wheel. $\theta$ is the angle between the dead center of the wheel and the point of study and $\theta_{e}$ is the entry angle defined by the position where a point on the wheel circumference first comes into contact with the terrain, which is related to sinkage (a high entry angle means high sinkage and vice versa) [Wong, 2008]. . . . 104

A.2 Simplified scheme of the driven wheel showing slip velocity and slip $i$. . . . . 105

A.3 Driven wheel with forces and stresses applied on a given point $K$ on the circumference. The angles between the different stresses and forces are given in the figure $[$ Muro, 2004] . . . . . . . . . . . . . . . . . . . . . . 107

A.4 Enlarged area from Fig.A.3 showing: (a) vectors $\overline{K T}$ and $\overline{K H}$, (b) angle between vectors and stresses and (c) shear and normal stresses added to the other stresses at a given point on the wheel $[$ Muro, 2004] . . . . . . . . . . . . . . 107

A.5 Wheel driving and motion on a $(X, Y)$ plane at a given point on the circumference, referred to as $G(X, Y)$. This point touches the ground at point $C, V$ is the longitudinal velocity and $R$ the radius of the wheel $[$ Muro, 2004] . . . . . . . . 108

A.6 Enlarged area from Fig.A.3 at point $K$ giving the geometry of $\tan \eta$. . . . . . . . 109

A.7 Compaction resistance $R_{c}$ shown for a driven wheel with the normal stress $\sigma_{n}$, sinkage $z_{0}$, and load $W$ for a driven wheel of diameter $D . d x$ and $d z$ are elements of contact area between wheel and soil at a studied point $K$ at a depth $z, q$ is vertical pressure, $B$ is the center of the wheel and $A$ is the vertical projection of $B$ at depth $z . V$ is the longitudinal velocity, $\Theta$ is the angle between the bottom of the wheel and point $K$ and $\theta_{e}$ is the entry angle [Bekker, 1969] . . . . . . . . . . . . . 111

A.8 Stresses and forces at point $K$ on the circumference of a driven wheel [Muro, 2004]. 112 


\section{List of Tables}

2.1 Presentation of the 17 terrain types as defined by SPOC (Rothrock, et al., 2016). All the images are credited to NASA JPL. . . . . . . . . . . . . . . . . . . . . . . 7

5.1 Principal Component Analysis (PCA) results showing PCs (Principal Components) 1 to 4 with the contribution of each variable (in \%) . . . . . . . . . . . . . . . 41

5.2 Slip probability distributions parameter, per velocity category, derived from Spirit's slip checks . . . . . . . . . . . . . . . . . . . . . . 48

5.3 Velocity categories and their characteristics for those with only one given number [Ono et al., 2018] . . . . . . . . . . . . . . . . . . . .

5.4 Confusion matrix for traversability classes ( 1 to 5 [Ono et al., 2018]) derived from Rothrock et al. (2016). Terrains included in the classes are: SR - Smooth Regolith, SO - Smooth Outcrop (class 1), RR - Rough Regolith, RO - Rough Outcrop (class 2), SRF - Sparse Ripples Firm, SRS - Sparse Ripples Sandy (class 3), DLR - Dense Linear Ripples, and PR - Polygonal Ripples (class 5). As specified before, no class 4 was detected at the Columbia Hills [Rothrock et al., 2016] . . . . . . . . . . . 51

$5.5 \mathrm{CFA} \leq 7 \%$ and $20^{\circ}<$ slope $\leq 25^{\circ} \ldots \ldots \ldots \ldots \ldots$

5.6 Prototype Hardware and Estimated Maximum Energy Consumption. Credit: D. Covell. . . . . . . . . . . . . . . . . . . 59

5.7 Parameters associated with each traversability class on soft terrains. Values are retrieved from the survey presented in Sullivan et al. (2011) . . . . . . . . . . . 60

6.1 Trials 1 to 4 (see Fig.6.1 for location). . . . . . . . . . . . . . . . . 73

6.2 Results of cohesion and angle of internal friction from testing. In green are adequate results, and in red, inadequate results. . . . . . . . . . . . . . 78

6.3 Results of cohesion and angle of internal friction from testing. . . . . . . . . 80

6.4 Estimated ultimate bearing capacity for various soils derived from the Mohr circles at the point of failure. . . . . . . . . . . . . . . . . 82

A.1 Parameters used in Appendix A. . . . . . . . . . . . . . . . . . . 102

B.1 CFA $\leq 7 \%$ and slope $\leq 10^{\circ} \ldots \ldots \ldots \ldots \ldots \ldots \ldots$

B.2 CFA $\leq 7 \%$ and $10^{\circ}<$ slope $\leq 15^{\circ} \ldots \ldots \ldots \ldots \ldots \ldots$

B.3 CFA $\leq 7 \%$ and $15^{\circ}<$ slope $\leq 20^{\circ} \ldots \ldots \ldots \ldots \ldots \ldots$

B.4 CFA $\leq 7 \%$ and $20^{\circ}<$ slope $\leq 25^{\circ} \ldots \ldots \ldots \ldots \ldots \ldots$

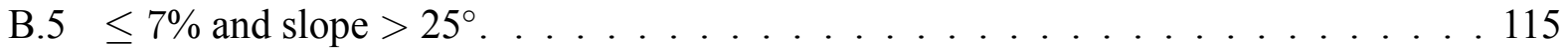

B.6 $\leq 7 \%$ and $10^{\circ}<$ slope $\leq 15^{\circ} \ldots \ldots \ldots \ldots \ldots \ldots$

B.7 $7 \%<\mathrm{CFA} \leq 15 \%$ and slope $\leq 10^{\circ} \ldots \ldots \ldots \ldots$ 
B.8 $7 \%<\mathrm{CFA} \leq 15 \%$ and $10^{\circ}<$ slope $\leq 15^{\circ} \ldots \ldots \ldots \ldots \ldots$

B.9 $7 \%<\mathrm{CFA} \leq 15 \%$ and $15^{\circ}<$ slope $\leq 20^{\circ} \ldots \ldots \ldots \ldots \ldots$

B. $107 \%<\mathrm{CFA} \leq 15 \%$ and $20^{\circ}<$ slope $\leq 25^{\circ} \ldots \ldots \ldots \ldots \ldots$

B.11 CFA $>15 \%$ and any slope. . . . . . . . . . . . . 116 


\section{LIST OF SYMBOLS/NOMENCLATURE}

ARTEMIS Adams-based Rover Terramechanics and Mobility Interaction Simulator

BRM Belief Roadmap

CFA Cumulative Areal Fraction

CRISM Compact Reconnaissance Imaging Spectrometer for Mars

CTX Context Camera

DESPOT Determinized Sparse Partially Observable Tree

DSN Deep Space Network

DTM Digital Terrain Model

EDL Entry Descent Landing

EST Expensive Search Tree

FIRM Feedback-based Information Roadmap

FTR Fast Traverse Rover

HiRISE High Resolution Imaging Science Experiment

IMU Inertial Measurement Unit

ISTVS International Society on Terrain-Vehicle

JPL Jet Propulsion Laboratory

MAV Mars Ascent Vehicle

MDP Markov Decision Process

MEPAG Mars Exploration Program Analysis Group

MER Mars Exploration Rover

MOMDP Mixed Observability Markov Decision Process

MRF Markov Random Field 
MRO Mars Reconnaissance Orbiter

MSL Mars Science Laboratory

MSR Mars Sample Return

MTTT Mars Terrain Traversability Tool

PBVI Point-Based Value Iteration

PCA Principal Component Analysis

POMCP Partially Observable Monte Carlo Planning

POMDP Partially Observable Markov Decision Process

PRM Probabilistic Roadmap

RA-L Robotics Automation Letters

RIG algorithm Rapidly exploring Information Gathering algorithm

ROI Region of Interest

RPP Randomized Potential Planner

RRBT Rapidly exploring Random Belief Tree

RRT Rapidly exploring Random Tree

SARSOP Successive Approximations of the Reachable Space under Optimal Policies

SPOC Soil Property and Object Classification

TES Thermal Emission Spectrometer

TI Thermal Inertia

TRN Terrain Relative Navigation

UGM Undirected Graphical Model

USCS United Soil Classification System

VFF Virtual Force Field

VFH Vector Field Histogram

WVUIRL West Virginia University Interactive Robotics Laboratory 


\section{CHAPTER 1 Goal and High-level Statement}

NASA has launched its next mission to Mars, which includes a rover similar to Curiosity named Perseverance. The rover is designed to collect samples with the intent of bringing them back to Earth during the following mission. There is thus a proposed Mars Sample Return mission whose sole purpose is to bring the samples back.

West Virginia University's Interactive Robotics Laboratory (WVUIRL), along with other laboratories at WVU, is proposing to develop an autonomous rover capable of going from one start point to a target and back, safely, to support efforts made at NASA towards a sample return mission. The goal is to traverse the distance between the Mars Ascent Vehicle (MAV) and the samples, which could be up to ten kilometers, rapidly (the rover does not need to stop for science unlike current and past missions), safely, efficiently and with limited human intervention.

This research primarily focuses on the terrain along the path the rover takes to get to the samples. Terrain analysis and path planning are deeply interconnected, and this work proposes a planning method that fully integrates the environment to achieve better performance. The main goal is to create a rover that has a high level of autonomy and can tackle the challenges related to a sample return mission as proposed by NASA.

There are three objectives to this research. The first objective consists of assessing the Martian terrain prior to landing. This would allow a high-level analysis of traversability (i.e., ability for a terrain to support a driving vehicle without reaching the failure point, which depends on the robot itself as well as terrain properties [Papadakis, 2013]) at a given landing site and detection of potentially dangerous areas ahead of time. The second objective consists of planning the traverse one day at a time (i.e., at local scale, up to $800 \mathrm{~m}$ as a daily driving objective) from one waypoint to another with options to replan to ensure safety. Using orbital information available at the landing site (e.g., topography) as well as an analysis of the rover's surrounding terrains during driving, the idea is to follow a path within a set time budget, while maintaining an accurate map of the environment. The third objective is about providing the rover with means to assess and identify a terrain autonomously, so as to avoid mobility problems during the traverse. If an unexpected situation and/or a danger is detected, there needs to be a method for the rover to decide what to do or where to go next. More specifically, this third part focuses on in-situ terrain monitoring as an asset to path planning.

The main contribution of this work is to support an autonomous, fast traversing rover capable of integrating its environment into its planning on a daily basis. This research develops a path planning algorithm at local scale (driving objective of a Martian day or sol) that fully takes the terrain into account prior to and during planning.

The rest of the dissertation is organized as follows: after presenting the motivation behind this research and background information related to the project, there will be a review of relevant literature. The technical approach will follow, and the integrated results will be presented, while future work will be mentioned at the end. 


\section{CHAPTER 2 Introduction}

\subsection{Motivation}

\subsubsection{Missions to Mars}

NASA's most recent guidelines are to send humans to Mars by 2033 [Chapman, 2020]. This lays out a variety of challenges ahead, such as building a habitat or supporting manned missions with limited input from Earth.

NASA's spacecrafts reached Mars decades ago with the Viking mission that arrived at the surface in 1976 [Soffen and Young, 1972]. It successfully landed a rover on Mars in 1997, called Sojourner [Matijevic, 1997] on board the Pathfinder mission. NASA has since sent the twin rovers Spirit and Opportunity that landed in January 2004 [Crisp et al., 2003], with Opportunity lasting more than a decade (it was declared lost in February 2019). In the meantime, Mars Science Laboratory, MSL, was launched to Gale Crater to explore the habitability of Mars [Grotzinger et al., 2012]. As technology improves, missions become more complex and goals are set higher. Having images back from Viking was an accomplishment in itself at the time; nowadays, with Curiosity, chemical experiments are performed on board. From a rough picture of the surface of Mars, technology has now made it possible to collect samples and analyze their composition directly from the surface of Mars. NASA has already launched its very next mission to the red planet, Perseverance, and has proposed the Mars Sample Return (MSR) rover set to bring back samples collected by the Curiosity-like rover [Witze, 2014].

\subsubsection{Mars Sample Return (MSR) and the proposed fetch rover}

The next proposed mission to Mars involves a fetch rover. Perseverance launched with onboard instruments capable of collecting samples, and the following mission, MSR, would retrieve them. The sample return proposal brings a new level of complexity to engineers: it is mostly designed to bring back samples (vs. performing science experiments in-situ), unlike any other missions to the red planet. Its current timeline is set to be within one Martian year (roughly equivalent to two Earth years) [MEPAG, 2008], ideally no more than 200 sols [Klein et al., 2014]. The landing ellipse for Mars 2020 is roughly $11 \mathrm{~km}$ by $8 \mathrm{~km}$ [Golombek et al., 2017], and assuming MSR will have the same landing area, then it might traverse potentially great distances in a short amount of time (although it is not required to stop for science, its purpose being to retrieve samples). The mission calls for more autonomy beyond the pre-planning done by humans and this can be seen at different levels: autonomy can be gained in path planning, navigation, decision making, etc... NASA has begun to work towards this goal of enhanced autonomy by implementing a Terrain Relative Navigation (TRN) software on board Perseverance to detect hazards during Entry, Descent, Landing (EDL), to identify science targets to land next to and to avoid traversing long distances [Johnson et al., 2015]. 
This research focuses on a proposed fetch rover and its autonomous capability to support NASA's effort towards a sample return mission. More specifically, the terrain and path planning components are extensively studied to propose a framework for such a mission.

\subsubsection{More autonomy needed for MSR and future space missions}

Future space missions, including MSR, will become more complex and some will go further into the solar system, where no one has gone before. There is proposed research for a journey to Uranus and Neptune [Mousis et al., 2018]. There are also proposed missions towards Saturn's moon Titan [Lorenz et al., 2018]. These missions require autonomy in robotics, as it would enable going further than line-of-sight moving, ensure safety at every step, and allow more complex designs to be put together with restricted budget and staffing [Fong et al., 2017].

To understand better how autonomy is becoming a necessary part of NASA's future missions, it is important to know how the past and current Mars missions are organized in terms of daily planning. Opportunity and Curiosity will be taken as examples to illustrate tactical operations. A typical sol (Martian day, about 40 minutes longer than on Earth) includes one downlink and one uplink through the Deep Space Network (DSN), which means that Earth communicates with Mars only a couple of times a day [Bajracharya et al., 2008]. The satellites around Mars act as a relay to get the data to and from the rover at the surface. A "Direct-to-Earth" (DTE) transmission is possible, however the amount of data transmitted is so limited that the team uses it as a backup in instances where the relays are down (satellite in safe mode, etc...). The principle of communication with the rovers is illustrated in Fig.2.1.

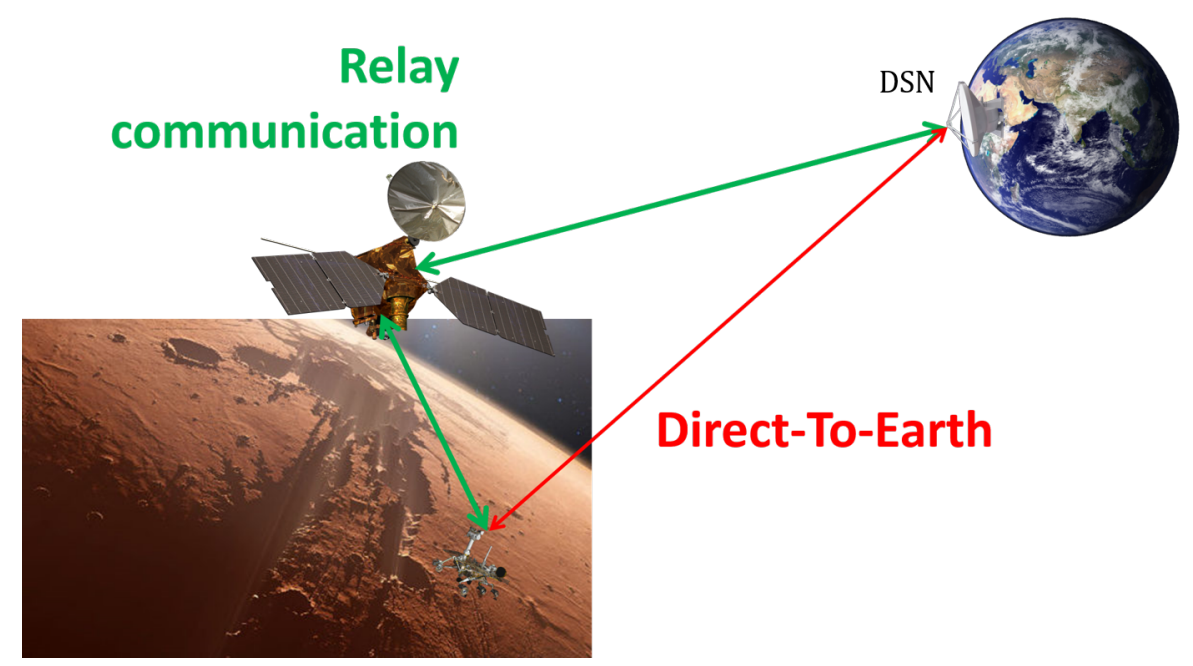

Figure 2.1: Communication between mission control on Earth and the rovers on Mars via satellites such as Mars Reconnaissance Orbiter (MRO) and the Deep Space Network (DTE).

The downlink provides the engineering team with images and other results from the previous sols that they can use to navigate. It should be noted that Curiosity differs from Opportunity in the sense that most of the time it undergoes tactical operations every day, therefore sols are planned one after the other (except on weekends). Opportunity's tactical planning, on the other hand, hap- 
pened every other day most of the time (restricted planning), and occasionally every day (nominal planning), making it even more difficult to plan, since data received were not even from the sol before. This difference impacts driving: if the rover was scheduled to drive on sol X, then the data from the drive (distance actually driven and current position) are not available to the team until downlink on sol $X+2$. Therefore, if planning occurred for sol $X+1$, the team still did not know where the rover was at the time of planning, how far it had driven, and had to go off of results from sol X-1. This meant that Opportunity could only drive at most twice in a week, whereas Curiosity can move more often because of daily tactical planning. Communication with Mars, including its frequency is thus the first limiting factor for surface missions.

Moreover, rover movement is limited to line-of-sight driving: when a drive is scheduled, the team simulates the rover's motion first, using available images, before sending the command to Mars. The maximum distance driven in one sol can go up to $200 \mathrm{~m}$, but has never been achieved - to date, the longest drive is just under $143 \mathrm{~m}$ (Curiosity on sol 665, [NYT, 2020]) - and the average distance is $40 \mathrm{~m}$ per drive. Although both Curiosity and Opportunity have the capability of driving autonomously if needed, as opposed to directed, the distances covered under autonomous navigation "Autonav" mode are even less than for directed driving. Indeed, when the team can assess the safety of the terrain via images prior to the drive, they can program the rover to "blind" drive, i.e. without checking the surroundings first. This implies that the rover remains within lineof-sight (i.e. path visible on pictures) and for MERs, it enabled speed up to $124 \mathrm{~m} / \mathrm{h}$ [Biesiadecki et al., 2007]. With Autonav, the rover takes sets of pairs of stereo images to map hazards or rough terrain, which slows down the rover given its limited computational power; MER speeds could be between $10 \mathrm{~m} / \mathrm{h}$ and $36 \mathrm{~m} / \mathrm{h}$ with Autonav enabled [Biesiadecki et al., 2007]. Therefore, to cover the same distance, Autonav will take a lot longer than a directed drive, possibly running into the energy limitation before even completing a drive. Usually drives are not solely Autonav and only a small portion is, as illustrated by sol 376 when Curiosity drove $10 \mathrm{~m}$ autonomously out of 43 $m$. MSL took almost three years to complete $10 \mathrm{~km}$, and Opportunity drove an average of $3 \mathrm{~km}$ in one Earth years [Schroeder, 2019]. For a mission such as the proposed fetch rover, that would potentially have to drive several kilometers in a few months, these modes would be inadequate. This leads to the following statement: there is a need to understand the terrain better to help future missions become more autonomous.

\subsubsection{The importance of understanding the terrain for planning}

Despite having remote sensing data for Mars, including high resolution images $(25 \mathrm{~cm} / \mathrm{px}$ for HiRISE, High Resolution Imaging Science Experiment [McEwen et al., 2007]) it is insufficient for planning purposes [Gaines et al., 2016] as it does not capture current conditions at the surface and translates into difficulty in preparing for day to day robotics operations. For example, upon arriving at the Columbia Hills, Spirit encountered highly deformable soil that had not been detected in orbital images (nor in ground data) and made traversability a challenge. [Johnson et al., 2015]. The rover underwent high sinkage (up to $10 \mathrm{~cm}$ ), and the failure of the right front wheel actuator made the traverse even worse, as Spirit was forced to drag its wheel along. It eventually got embedded and stuck in a sand-filled crater. Moreover, these soils were covered in basaltic sand, making them hidden on images, and it eventually led to the rover getting embedded [Johnson et al., 2015]. 
Contact was officially lost following this incident in 2010. Opportunity encountered high wheel sinkage situations at Endeavour Crater [Arvidson et al., 2011], and Curiosity experienced mobility difficulties with wheel damage (holes and dents) from roving on sharp rocks [Arvidson et al., 2017]. The importance of understanding terrain properties prior to driving has since been widely recognized. With MSR's timeline being within one Martian year (687 days) [MEPAG, 2008] and its main purpose being to retrieve samples up to $10 \mathrm{~km}$ away [Golombek et al., 2017], it is even more important to enable the rover to account for the terrain autonomously. The integration of soil information into the planning phase both offline and online could allow the rover to safely perform more frequent drives (potentially every sol) over longer distances than previous rovers and therefore maintain its mission timeline.

\subsection{Terrain related background information available to the fast traverse rover}

This section explores the background work performed on autonomy and traversability for future Mars missions that constitutes the foundation of this research. Path planning can be done on different levels, including: global (prepared planning from start to goal) and local (planning from one waypoint to the other along the path). The very first step of planning therefore involves understanding the terrain from orbit and obtaining as much information about traversability as possible. This includes obtaining topography, detecting obstacles such as craters and rocks (although not all can be detected from orbit) and analyzing terrain types to estimate the performance of the rover and plan the entire traverse. Both processed orbital imagery and ground assessment methods are extensively utilized in this work and are presented in the subsequent sections.

\subsubsection{Processed orbital analysis to support traversability assessment}

\subsubsection{Cumulative Fractional Area (CFA)}

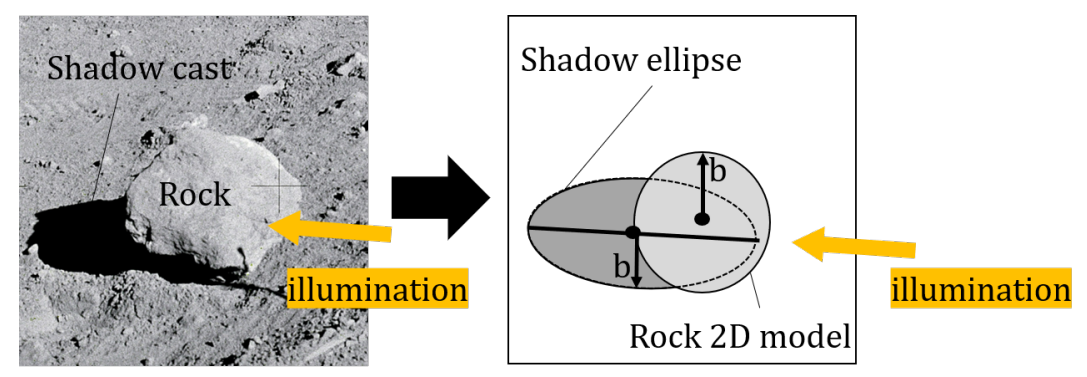

Figure 2.2: Best fit ellipse and cylinder to estimate shadow and rock sizes [Golombek et al., 2012, Golombek et al., 2008]. Photo credit:NASA.

One aspect of traversability assessment is the rock abundance along the traverse. As seen with Curiosity, rocks can be detrimental to the rover by causing damage (e.g., holes and dents) to the wheel [Arvidson et al., 2017] and/or can render the traverse difficult [Golombek and Rapp, 1997]. Studies have been made to estimate the size-frequency distribution of rocks on Mars and the Cumulative Fractional Area (CFA) covered by rocks of a given size and larger, using ground truth 
(i.e., rock sizes and diameters as seen on images) from the Viking missions. The derived model is presented in Eq.2.1 [Golombek and Rapp, 1997]:

$$
F_{k}(D)=k e^{(-q(k) D)}
$$

where $F_{k}$ is the cumulative fractional area covered by rocks of a given diameter $D$ ( $D$ in meters), $k$ is the cumulative fraction of surface covered by rocks of all sizes or the total rock coverage, and $q(k)$ is the exponential factor for Mars given by Eq.2.2 [Golombek et al., 2003]:

$$
q(k)=\left(1.79+\frac{0.152}{k}\right)
$$

The findings were later used to assess landing sites and the potentiality for hazards of a certain size and bigger via orbital imagery [Golombek et al., 2003, Golombek et al., 2017]. Overlapped images such as HiRISE (High Resolution Imaging Science Experiment) were used and rocks were detected using the shadows the rocks cast on these images. An ellipse is fit to the shadow and the rock size is estimated by approximating its shape to a cylinder with a diameter equal to the width of the ellipse, as shown in Fig.2.2. Its height is the length of the ellipse projected onto the illumination ray in combination with the Sun incidence angle [Golombek et al., 2008].

Several images covering the same regions are analyzed for rock detection, with techniques such as blind deconvolution, shadow enhancement or shadow decomposition to process the images with high accuracy and derive the rock abundance over selected areas [Golombek et al., 2003, Golombek et al., 2008, Golombek et al., 2017]. Results are fit to a size-frequency rock distribution to retrieve the Cumulative Fractional Area (CFA) or area covered by rocks of specific diameters and bigger, which gives the possibility of generating maps of rock density, for rocks of minimum sizes. When no minimum size is specified, CFA corresponds to the density of all rocks over a chosen area.

\subsubsection{Terrain classification software}

Analyzing the terrain is another important part of traversability assessment and path planning at both global and local scale. NASA JPL has developed a software capable of classifying terrains on Mars [Rothrock et al., 2016]. SPOC, the Soil Property and Object Classification, uses different types of data to classify terrains:

- HiRISE (High Resolution Imaging Science Experiment) images. These pictures are taken from orbit on board MRO. The resolution is $25 \mathrm{~cm} / p x$ (SPOC-H). This version of the software is used for Perseverance.

- Navcam images from the surface (Curiosity, Spirit and Opportunity) (SPOC-G). This version is geared towards predicting slip from the surface and is currently used for MSL. Terrains are split into 17 different categories [Rothrock et al., 2016] labeled from 0 to 16 [Rothrock et al., 2016]. Initially only 11 terrain types were identified, but this classification has been refined to account for an extra five types. This classification is used to determine traversability for a rover and are the foundation on which this work was built. The performance of the classifier as published in Rothrock et al. (2016) has been widely referenced throughout this research. 
Table 2.1: Presentation of the 17 terrain types as defined by SPOC (Rothrock, et al., 2016). All the images are credited to NASA JPL.

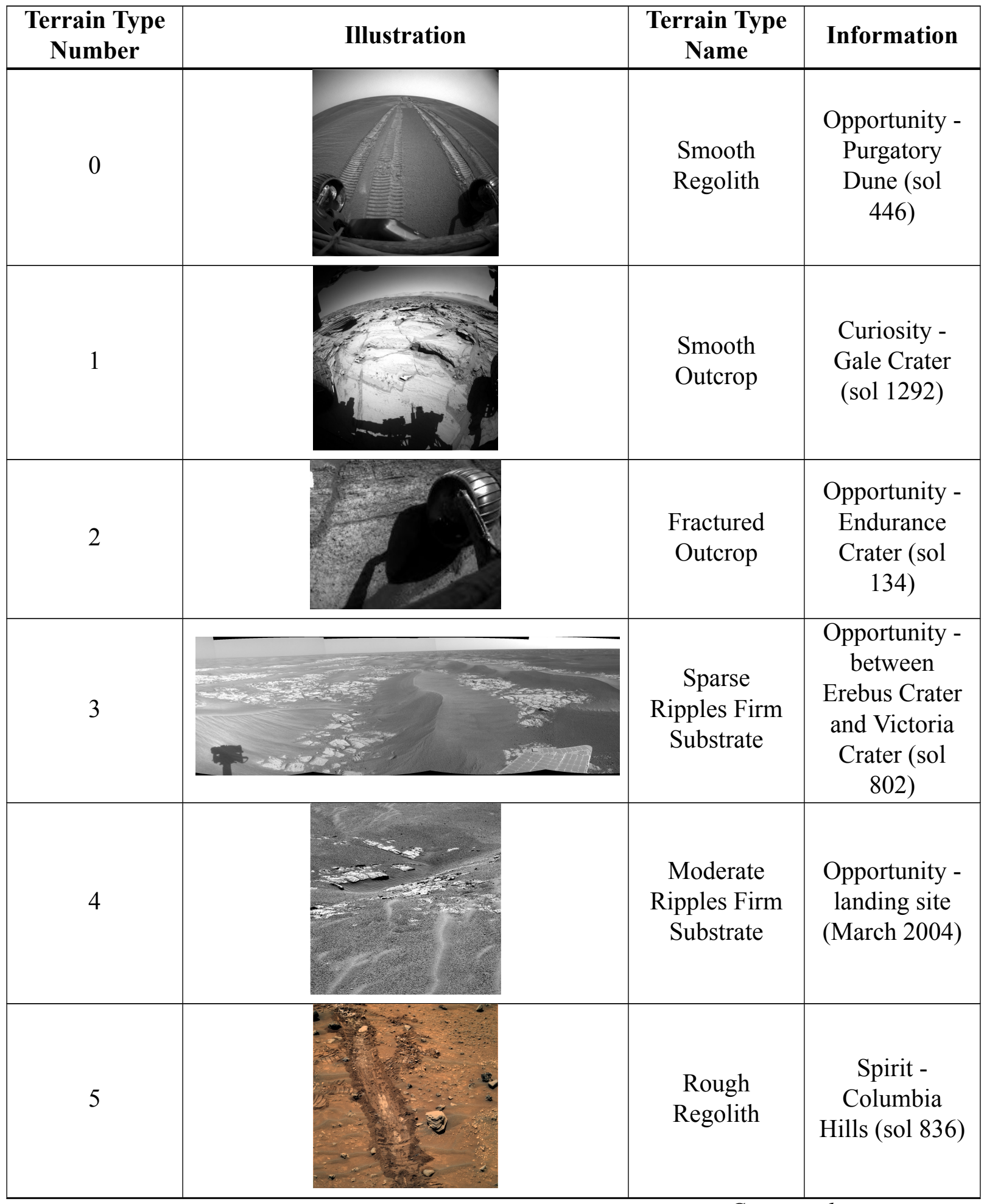

Continued on next page 
Table 2.1 - Continued from previous page

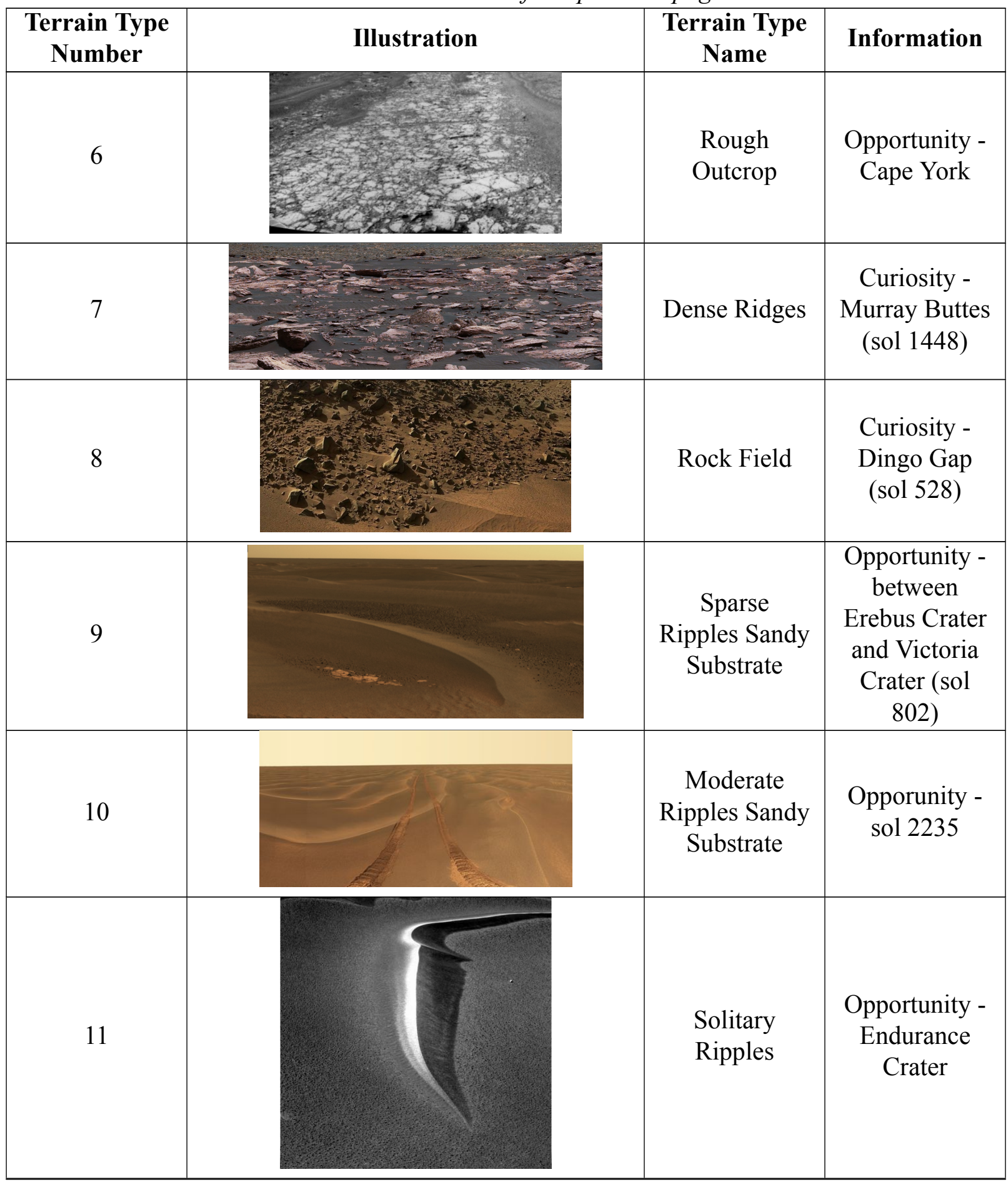

Continued on next page 
Table 2.1 - Continued from previous page

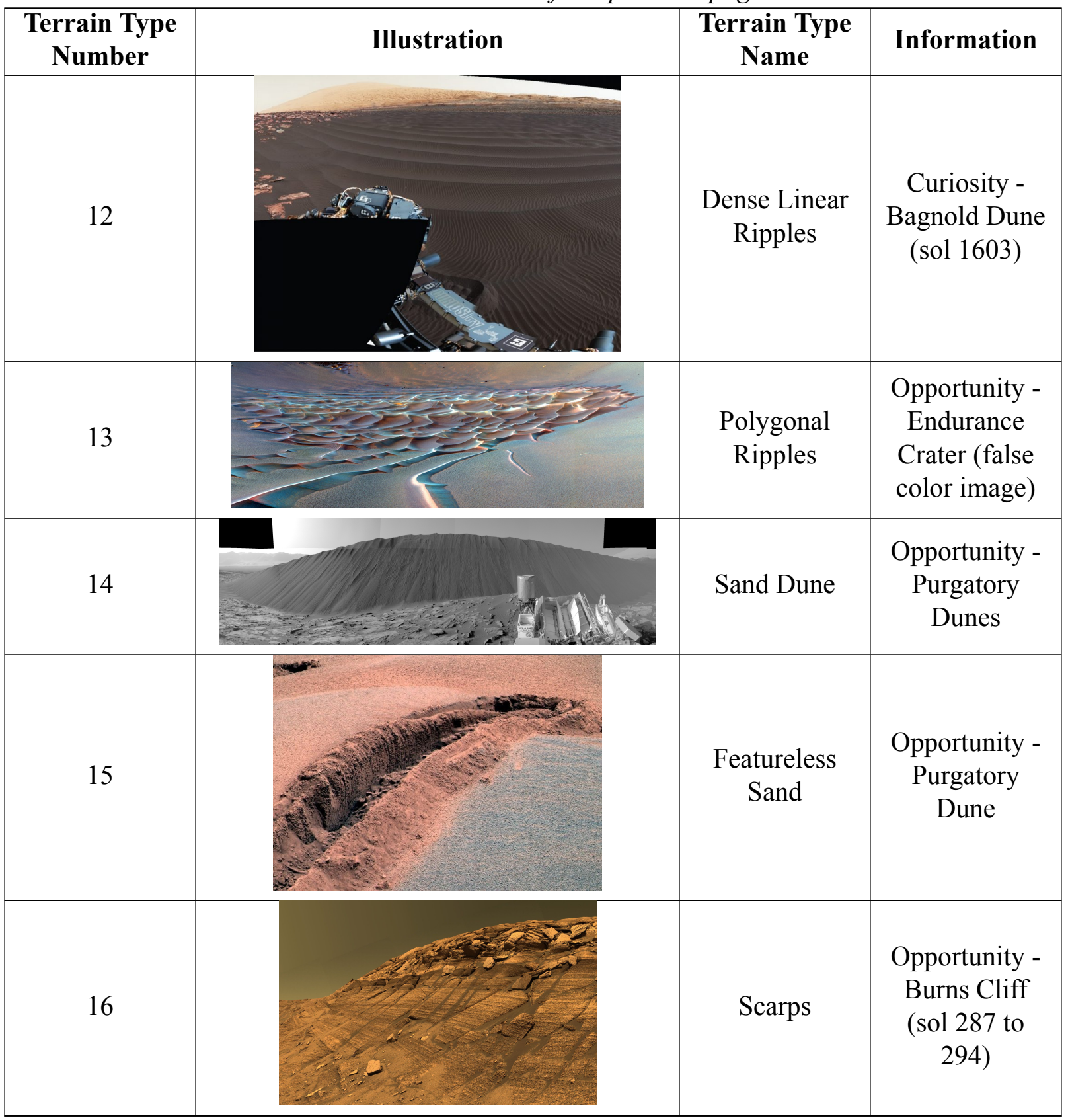

\subsubsection{Tool to determine traversability on Mars}

Once the terrain types are established, or at least an estimate of the terrain types, the fetch rover needs to know when to safely traverse. For example, terrain 16 is not traversable, but terrain 0 is easy to drive on. This is an essential part of path planning, as the algorithm should include a way to predict the traversability of each terrain before planning a path. To go even further, traversability can be scaled in terms of efficiency of driving. Two terrains can be safely traversable, but one 


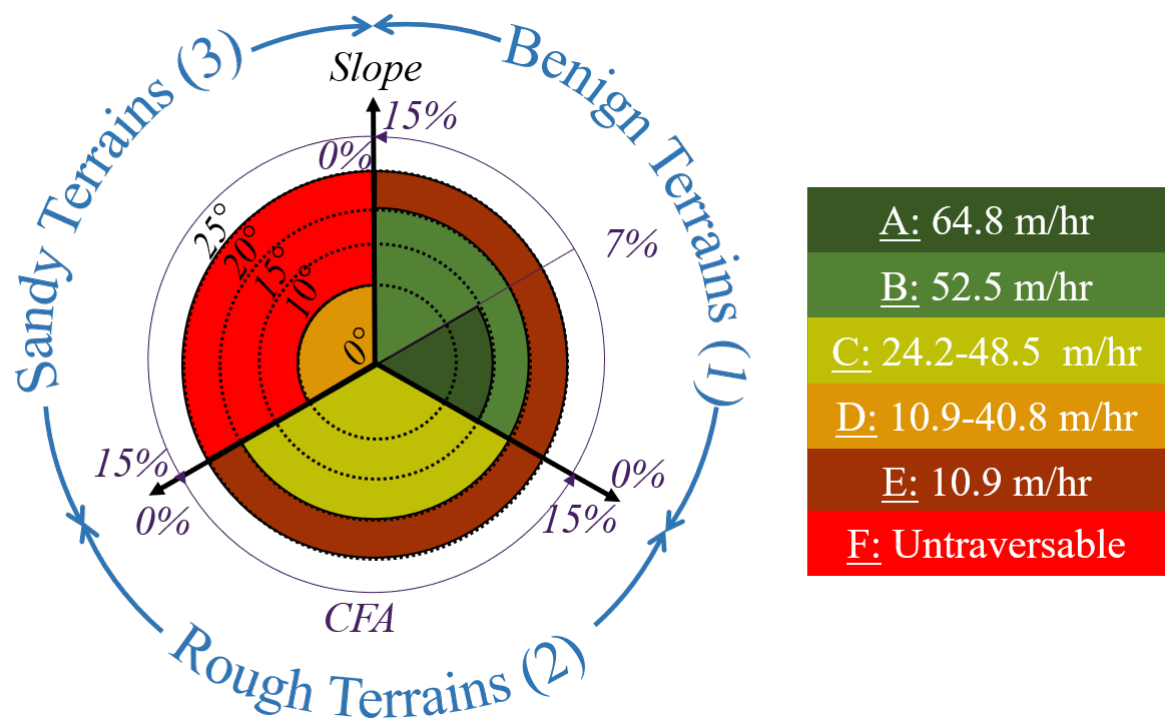

Figure 2.3: Traversability classes output from MTTT [Ono et al., 2018]. Credit: Hedrick et al., 2020.

would be easier to drive on than the other, therefore allowing the rover to drive longer distances or use less energy for a given time limit. This is the very reason why JPL has developed a software aimed at helping the engineering team assess the surface mobility of a Curiosity-like rover on Mars. It also brings in a more autonomous way to determine traversability. MTTT, the Mars Terrain Traversability Tool, uses HiRISE and CTX (Context Camera) to output traversability maps. The software generates a map of terrain types using SPOC, analyzes slope via Digital Terrain Models (DTM), calculates the CFA (Cumulative Fractional Area), takes into account a manual traversability assessment, and generates a hazard map using ROIs (Regions of Interest). The outputs are a map of traversable pixels, and a map of estimated traverse speed [Ono et al., 2018]. MTTT has been further developed to account for new terrain types implemented by SPOC and to more precisely model drive rates for each traversability class [Ono et al., 2018], as shown in Fig.2.3.

\subsubsection{Terrain parameters to support traversability assessment}

Beyond orbital analysis of the landing area to understand traversability and support global planning, there is a need to confirm the safety of the terrain from the surface. This section explores work on soil properties that can be used towards understanding the terrain locally.

\subsubsection{Terrain properties indicative of traversability}

There are three parameters that are important to traversability, as they characterize soil strength, which can directly affect driving: cohesion, angle of internal friction and ultimate bearing capacity.

Cohesion $c$ and angle of internal friction, $\phi$ are intrinsic parameters characterizing soil strength, a property crucial to a moving vehicle. Such parameters can also be used to classify planetary materials [Sullivan et al., 2011]. Cohesion is the ability of a material to hold itself together and corresponds to the cohesive strength of a terrain. The angle of internal friction gives an 


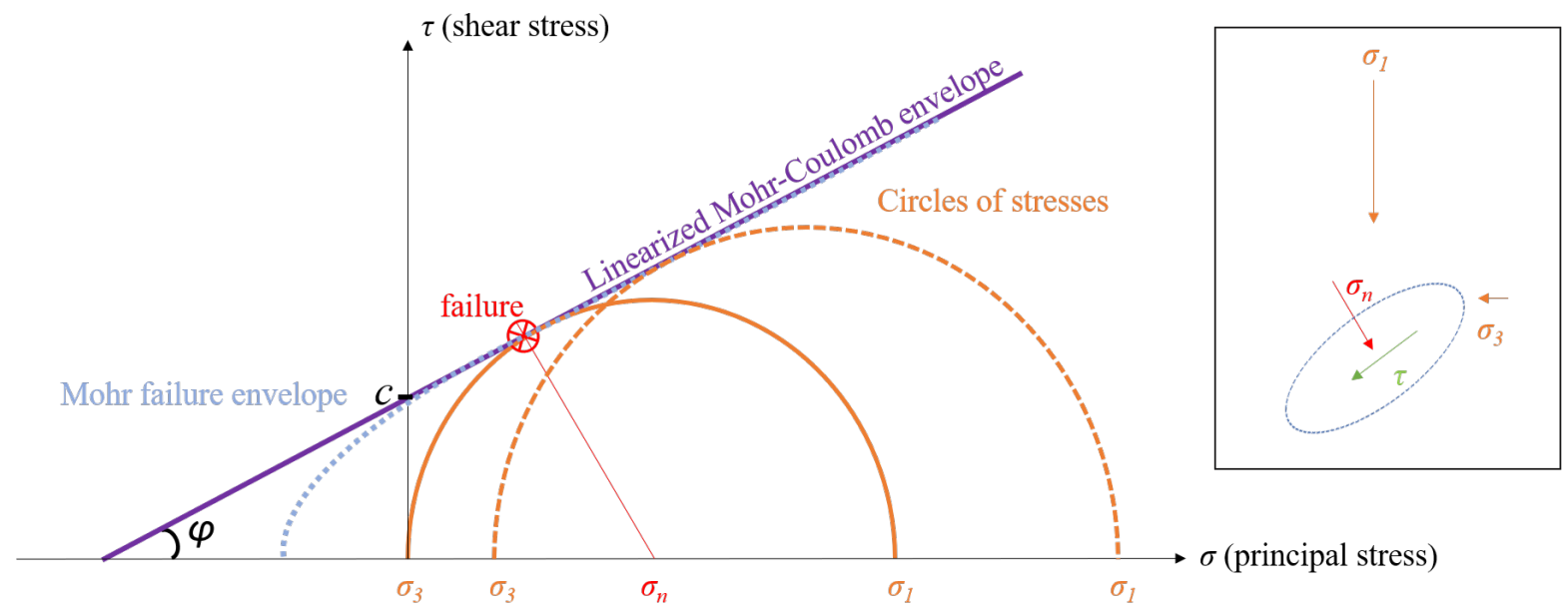

Figure 2.4: Mohr circles from principal stresses $\sigma_{1}$ and $\sigma_{3}$ leading to the Mohr-Coulomb envelope and failure point.

estimate of the friction due to the material itself. For example, a terrain with zero cohesion, such as loose rock debris, will still resist deformation due to friction, unless a stress is applied [Melosh, 2011]. Cohesion and friction are related to the normal and shear stresses via the following equation:

$$
\tau=c+\sigma_{n} \tan \phi
$$

where $\tau$ is the maximum shear stress the soil can handle, and $\sigma_{n}$ is the normal stress. Eq.2.3 represents the linear approximation of the Mohr-Coulomb failure envelope [Zoback, 2010]. The intersection of the Mohr-Coulomb failure line with a circle of stresses (given by $\sigma_{1}$, vertical stress and $\sigma_{3}$, confining pressure) determines the point of failure for this specific set of stresses $\left(\sigma_{1}, \sigma_{3}\right)$. The line perpendicular to the point of failure and intersecting the x-axis gives the normal stress $\sigma_{n}$ (Fig.2.4).

The bearing capacity can be defined as either allowable or ultimate. Both are used in civil engineering to determine the capacity of a soil to bear a structure. The allowable bearing capacity $q_{a}$ is the maximum load of stress that can be applied on a terrain that will assure safety against shear failure and does not exceed the maximum tolerable settlement. The ultimate bearing capacity $q_{u l t}$ is the maximum load that can be applied to a terrain before shear failure, and is related to $q_{a}$ by a factor of safety $F_{s}$, usually between 2.0 and 4.0 [Brown, 1992]. In the case of Mars, knowing the ultimate bearing capacity could help prevent an unwanted failure, which has happened before with the Spirit rover (its wheel broke through a crusty layer of sand).

\subsubsection{Measurement of various soil parameters}

Direct methods to obtain $c$ and $\phi$ are implemented in a laboratory, where samples of soil are subjected to vertical stresses and confining pressure [Bishop and Henkel, 1962] in what is called a triaxial test. Direct methods to derive $q_{u l t}$ include dropping a weight and measuring the depth of the impact, or loading, where a plate of steel is placed on a leveled soil and settlement is measured until it ceases.

Indirect methods include deploying an instrument in-situ, such as a cone penetrometer or a shear vane. While the actual purpose of a penetrometer is to measure the soil compaction by pushing 
a pointed rod into the soil, it can give cohesion values for different angles of internal friction, as demonstrated with the lunar missions, for which the following equation was used [Heiken et al., 1991] :

$$
q_{c}=c N_{c} \xi_{c}+g_{l} B N_{\gamma q} \xi_{\gamma q}
$$

where $q_{c}$ is the cone resistance, $c$ is the cohesion, $\rho$ is the density of the lunar soil, $g_{l}$ is the acceleration of lunar gravity, $B$ is the width of the penetrometer, $N_{c}, N_{\gamma q}$ are the bearing capacity factors

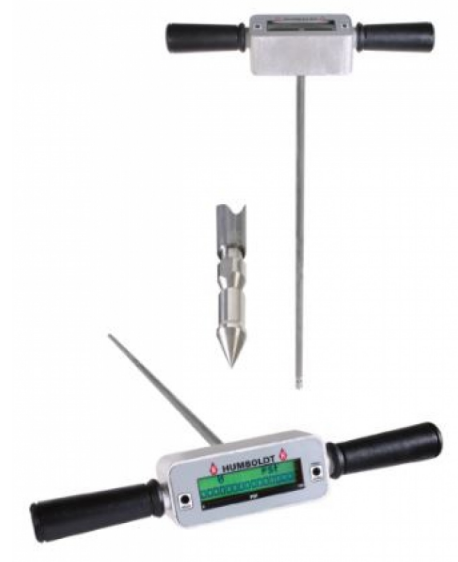

Figure 2.5: Digital Cone Penetrometer by Humbolt. (i.e., function of the angle of internal friction, cone roughness and coefficient of lateral stress), and $\xi_{c}, \xi_{\gamma q}$ are shape factors (functions of width, depth and length). The cone resistance $q_{c}$ (a.k.a. ground resistance) is retrieved from cone penetration tests by direct readings of the instrument (Fig.2.5) and is usually given in $\mathrm{kg} / \mathrm{cm}^{2}$. Values for cone roughness, coefficient of lateral stress and soil density were assumed [Mitchell et al., 1972], and curves of cohesion vs. angle of internal friction for lunar regolith were computed with these values.

The cone penetrometer can also give the bearing capacity. The relationship between cone resistance and bearing capacity has been extensively studied over the years [Eslami and Gholami, 2003] and there is a direct correlation between $q_{c}$ and the ultimate bearing capacity, established for footings as follows [Mayerhof, 1976]:

$$
q_{u l t}=\overline{q_{c}} \frac{B}{12.2}\left(1+\frac{D_{f}}{B}\right)
$$

where $q_{u l t}$ is the ultimate bearing capacity $\left(\mathrm{kg} / \mathrm{cm}^{2}\right), B$ is the width of footing $(\mathrm{cm}), \overline{q_{c}}$ is the average of $q_{c}$ values in a zone $\left(\mathrm{kg} / \mathrm{cm}^{2}\right)$, and $D_{f}$ is the depth of footing $(\mathrm{cm})$. In the case of a rover, the footing would be the area covered by the rover and its depth would correspond to the sinkage.

Another instrument selected for planetary missions to retrieve soil properties, called a cone vane, was used on the Lunakhod to obtain the bearing capacity of the regolith. The cone vane resembles a shear vane, which can provide the shear strength of a soil by application of a vertical load to a set of blades that rotate into the soil until it reaches the maximum shear strength (which corresponds to $\tau$ in Fig.2.4). There are three main types of shear vanes: the geovane, the field shear vane (Fig.2.6) and the pocket shear vane. The version that flew on board the Russian rover was a hybrid field shear vane and penetrometer, with blades mounted on
Figure 2.6: Field shear vane with extension rods and different sizes of blades. Credit: Humbolt. 
a rod displaying a pointed tip. The bearing capacity was calculated by taking the vertical load applied to penetrate the soil, divided by the area of the rod [Zacny et al., 2010]. 


\section{CHAPTER 3 Problem Statement}

As previously stated, the main purpose of this research is to develop the technology to support NASA in its effort towards the proposed MSR mission, a rover that would be able to drive to the samples left by Perseverance, currently on its way to the red planet, collect them and bring them back to the MAV (Mars Ascent Vehicle). This work focuses on local planning along the traverse, as well as the integration of terrain information into the planning phase.

The overall statement is presented as objectives, information available, constraints, assumptions, challenges and contributions.

\subsection{Objectives of the research}

The purpose of this research is to support the autonomous, terrain-aware traversal of a rover under Mars conditions for the driving objective of a sol. The detailed goals of this research include identifying expected traversability information prior to planning, finding a planning approach that balances offline and online methods to alleviate the use of the limited onboard computational resources, implementing a fast replanning method if needed, updating the traversability information along the drive and preparing for important discrepancies between what is expected and the actual environment. Overall, all these detailed purposes aim at ensuring the safety of the rover and the efficiency of the traverse.

This research is divided into three components, strongly connected to each other. The first component consists of generating a map containing traversability information for each pixel. The traverse from landing to samples is computed on this basemap. The second component is the planning of the path for one sol (about $800 \mathrm{~m}$ on average), including implementation of a terrain monitoring method to assess safety along the planned path, local update of the map and capability to replan if needed. The third component is related to the implementation of a method to obtain exact terrain information to support planning if simple monitoring techniques are not sufficient to ensure safety. This is summarized in Fig.3.1: the traversability information map is used to plan the path every sol, and a method to monitor/assess the terrain is implemented to support planning and keep the map of traversability information up to date.

\subsection{Information available to support this research}

Different types of information specific to Mars surface missions are available to the rover prior to its arrival on the planet. Jezero Crater was selected for Perseverance, and consequently, for MSR [NASA, 2018]. Usually, there is an extensive orbital coverage over candidate landing sites for detailed analysis and selection, which implies that orbital information such as HiRISE, 


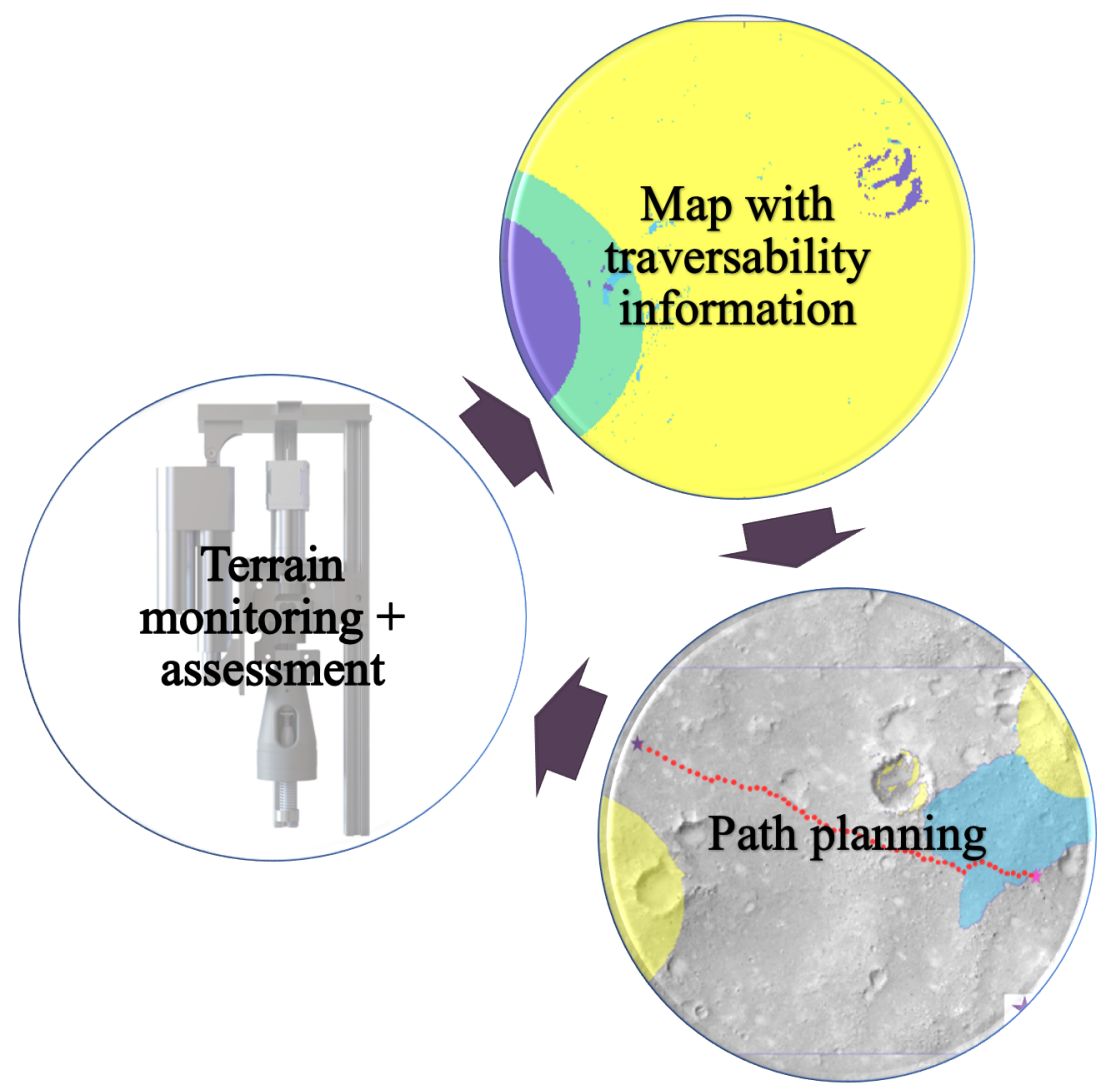

Figure 3.1: Approach to path planning given terrain information.

thermal images, topography, etc, are available at Jezero Crater. These orbital images can be utilized to generate the terrain type map as described in Rothrock et al. (2016), as well as a CFA map [Golombek and Rapp, 1997]. In the case of MSR, due to the nature of its mission (retrieve samples left by a previous rover), ground information will also be available, gathered by Perseverance. In order to conduct this study in conditions as close as possible to the actual sample return mission, it has been decided to perform simulations for this research using information from Gusev Crater, Mars, instead of Jezero Crater. As home to the rover Spirit, it presents today with similar conditions to what Jezero will present after Perseverance completes its mission. As Spirit's landing site, Gusev Crater and the Columbia Hills have orbital and ground coverage, and processed information such as terrain type and CFA maps are already available. Approaches and results can then easily be applied to Jezero in the future.

\subsection{Constraints related to Mars missions}

Several constraints that apply to missions in Mars-like environments need to be accounted for during all phases of the project, such as limited availability of data. This constraint is related to the type of data gathered by previous missions (surface and orbital) and the amount of data 
obtained from the various suites of instruments on board the spacecrafts. In addition, those data have different resolutions, which add to the constraints related to this work. Instruments sent to Mars have gathered data that range from $100 \mathrm{~m}$ (thermal inertia [Christensen et al., 2004]) to 25 $\mathrm{cm} / p x$ (HiRISE [McEwen et al., 2007]). This research is also constrained by NASA's current objective of a short timeline, leading to limited time to complete the mission [Klein et al., 2014]. Moreover, as seen with previous rovers, there are limited online computational resources [Carsten et al., 2007] that need to be taken into consideration.

In addition to environmental and mission constraints, other restrictions related to NASA and ITAR (International Traffic in Arms Regulations) regulations apply to certain data sets. Those will be identified throughout this research and solutions to account for them will be detailed.

\subsection{Assumptions applicable to this research}

Extensive work is being conducted at WVU regarding the fast traversal of a rover to support the proposed MSR, and several topics are taken as assumptions in this research since not they are not its direct focus, such as: known location (low uncertainty on localization); obstacle avoidance for obstacles not otherwise detected from orbit; and the ability to monitor its behavior such as wheel slip and actual speed.

Other assumptions are made to simplify the problems (without being subject to research at WVU). Such examples include a pre-determined path from landing to sample and the availability of daily communication with Earth. Some assumptions are made on the data sets as well: the terrains are classified by SPOC into a fixed number of types (17), which means that if it is found that one area is not of the expected type, then it has to be of one of the remaining 16. It is assumed that the performance of SPOC is the same everywhere on Mars (i.e., the uncertainties of SPOC published in Rothrock et al. (2016) over the Columbia Hills can be used at other locations), that uncertainties related to terrain classification (i.e., SPOC output) are given and applicable to this research and that it is the only data set to contain uncertainty (other orbital data sets, raw and/or processed, do not contain any errors).

\subsection{Challenges of a fast traversing sample return rover}

The map preparation involves using a variety of raw data not georeferenced, with different resolutions, and overlapping them to produce more useful information over the chosen landing. Once the map is generated, the path planning process must incorporate the information given by the map. Moreover, it should also contain an option for replanning if necessary, in real time, under budget and computational resources constraints if they are defined. For example, a time budget could be to drive $800 \mathrm{~m}$ in a single sol and would ensure that the overall timeline of the mission is respected, even though for past and current rovers this would be an unrealistic expectation [Biesiadecki et al., 2007]. Finally, gathering meaningful information about the terrain to help verify the traversability information ahead as well as update the map locally is another challenge that will be 
addressed in this study.

\subsection{Contributions of this work}

The contributions are detailed for each component of this work and can be summarized as follows: this research gives a planning framework that addresses challenges posed by Mars surface missions, specifically in the case of a sample return. It proposes a method to incorporate terrain information obtained from orbital analysis into the path planning and it gives efficient replanning steps while keeping the onboard computational cost to a minimum. It also implements processes along the traverse that confirm traversability of the terrain ahead of the rover. 


\section{CHAPTER 4 Literature Review}

\subsection{Introduction}

This Chapter focuses on reviewing the literature relevant to this project. As previously stated, this work can be divided into three components: generation of a terrain map, path planning and terrain assessment. The following areas will therefore be explored: path planning, terramechanics (interaction between a vehicle and the ground) and current research on planetary exploration.

\subsection{Path planning}

\subsubsection{Overview and definitions}

This part focuses on reviewing major path planning techniques relevant to the fetch rover problem. Robot path planning has been studied extensively for decades, and the algorithms developed are still being improved nowadays. The following definitions will be used in this section:

- The configuration space is defined as "a set of possible transformations that could be applied to the robot" [LaValle, 2006]. It is usually referred to as C-space.

- The free space, $C_{\text {free }}$, is the part of the configuration space that is free for the robot to move into and is collision free.

- The obstacle space, $C_{o b s}$, as indicated by its name, is all the configuration space occupied by obstacles.

Planning often involves a graph, which is a mathematical object of vertices and edges. A graph is defined by a pair $G=(V, E)$ of sets such that $E \subseteq[V]^{2}$ [Diestel, 2010]. $V$ are vertices or nodes, represented as dots, and $E$ are edges, connecting vertices together. A graph is a convenient way to simulate maps, where the vertices can represent $(x, y, z)$ coordinates, and edges would be the path from one vertex to another. The path planning problem thus becomes one of connecting edges to go from A to B as efficiently as possible (in other words, it aims at minimizing a cost function between $\mathrm{A}$ and $\mathrm{B})$.

\subsubsection{Sampling-based algorithms}

These types of algorithms use sampling to create a graph representation of C-space, typically when the map covers an extended area, or the robot requires modeling of certain constraints. There are many advantages to sampling-based algorithms: they can be implemented online, in real time; they do not require a representation of all the constraints and all C-space; and they adapt to different resolutions. This also implies that they are well suited for high dimensional problems.

In 1991, Barraquand and Lacombe published a leading paper proposing an RPP, Random- 
ized Potential Planner [Elbanhawi and Simic, 2014], an algorithm based on random walk used to escape minima within a potential field planner [Barraquand and Latombe, 1991]. As the name indicates, potential field planners use a potential function to model $C_{f r e e}$ and $C_{o b s}$. It works similarly to, for example, electrical potential. The idea is to create an artificial potential with goals modeled as minima and obstacles as maxima [Hwang et al., 1992]. This research led to modern samplingbased planners such as Probabilistic Roadmap (PRM, [Kavraki et al., 1996]) and Rapidly exploring Random Tree (RRT, [LaValle, 1998]), both widespread in robotics due to their easy implementation and the quality of their solutions [Elbanhawi and Simic, 2014].

The sampling process has seen different methods being published over the years, including: visibility graphs [Nilsson, 1969, Lozano-Pérez and Wesley, 1979], Delaunay triangulation [Lee and Schachter, 1980], Voronoi [Canny, 1985], or more recently, adaptive roadmap [Elbanhawi et al., 2013].

Since PRM and RRT are of significant importance, they are detailed below. They are widely used in path planning because of their ability to explore the state space rapidly and find a feasible solution that gets closer and closer to optimal with increasing computing time.

\subsubsection{Probabilistic Road Map (PRM)}

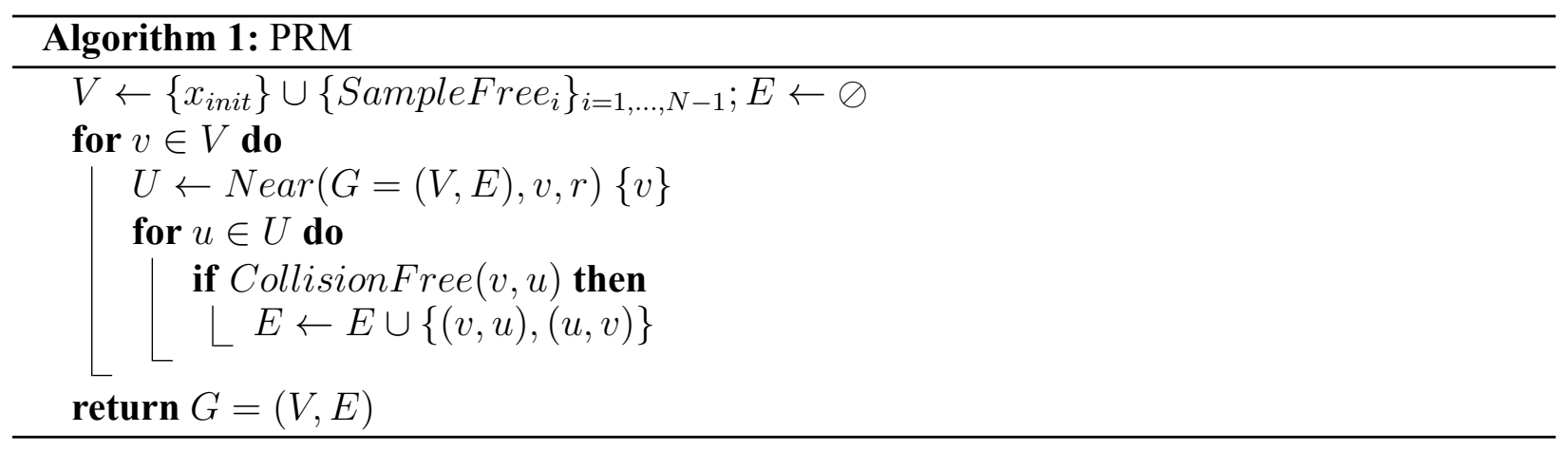

PRM [Kavraki et al., 1996] is implemented as follows: C-space is sampled randomly where there are no obstacles, creating the graph nodes, and each node is then linked by edges to its nearest neighbors, assuming such edges are in free space. It therefore creates a network of edges in $C_{\text {free }}$, all considered when searching for the path. The algorithm then links the source node to the goal node by travelling along the network of edges. Figure 4.1 illustrates the principle of the PRM code using a simple geometry. The pseudocode is given in Algorithm 1, where $V$ symbolized all the vertices $v$ of graph $G, E$ is the family of edges and $U$ is the family of new vertices $u$ added to the graph if collision free. The radius $r$ determines how far around the considered node the algorithm looks for neighboring points. This is the basic PRM, but there exist variants of this code, such as lazy PRM, which reduces the number of collision checks, therefore decreasing the running time [Bohlin and Kavraki, 2000]. This is one of many examples of PRM-based algorithms that are not detailed here.

\subsubsection{Rapidly exploring Random Tree (RRT)}

In RRT [LaValle, 1998], a tree whose branches are edges is grown from a predefined source node. A random sample is chosen within $\mathrm{C}$-space, and the algorithm then searches for the closest 

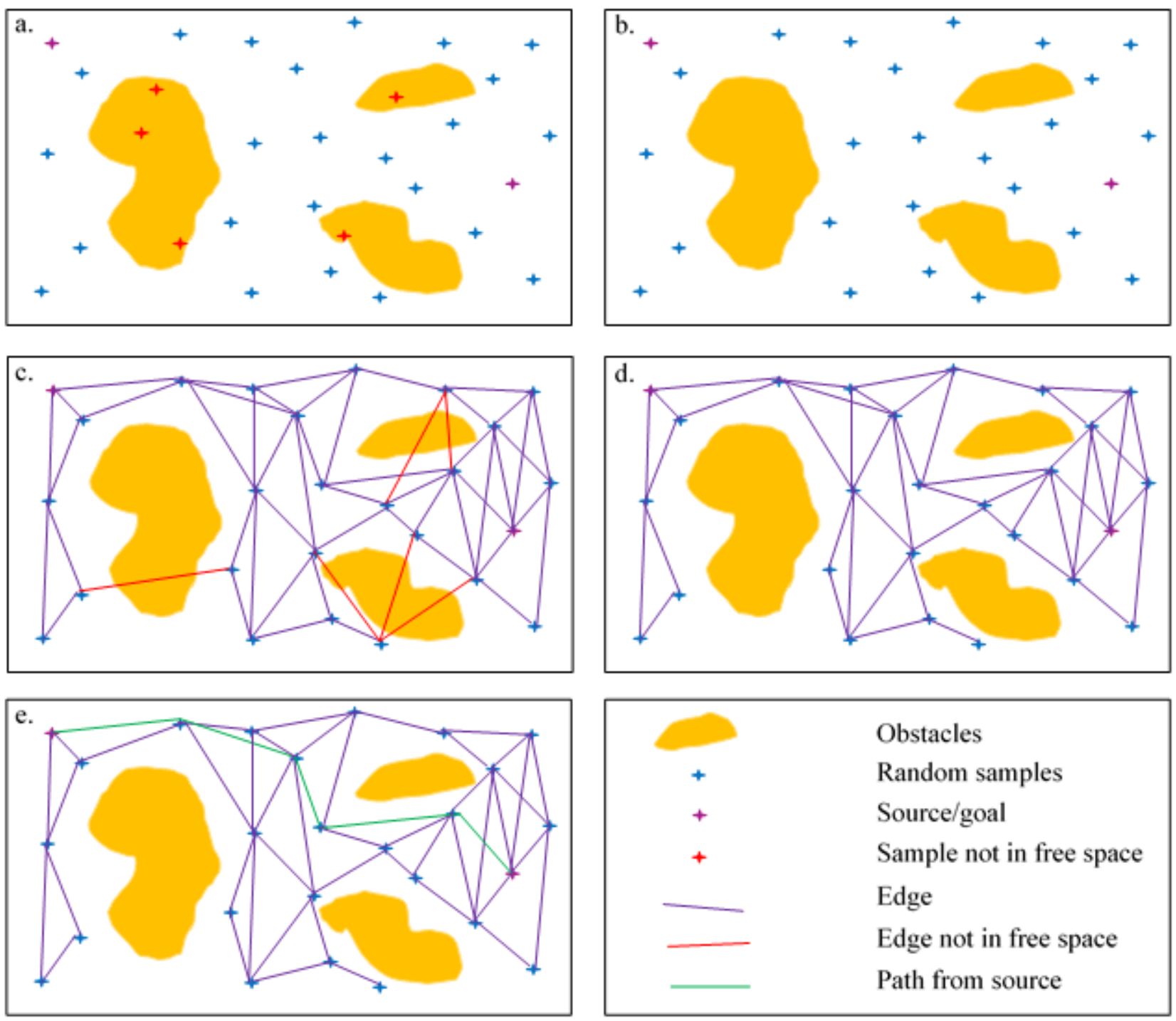

Figure 4.1: Illustration of the implementation of a PRM algorithm: (a) C-space is sampled randomly, and (b) samples are checked for collision. (c) nodes are connected to their nearest neighbors and (d) edges are checked for collision. (e) the path from start to goal is found using the remaining edges.

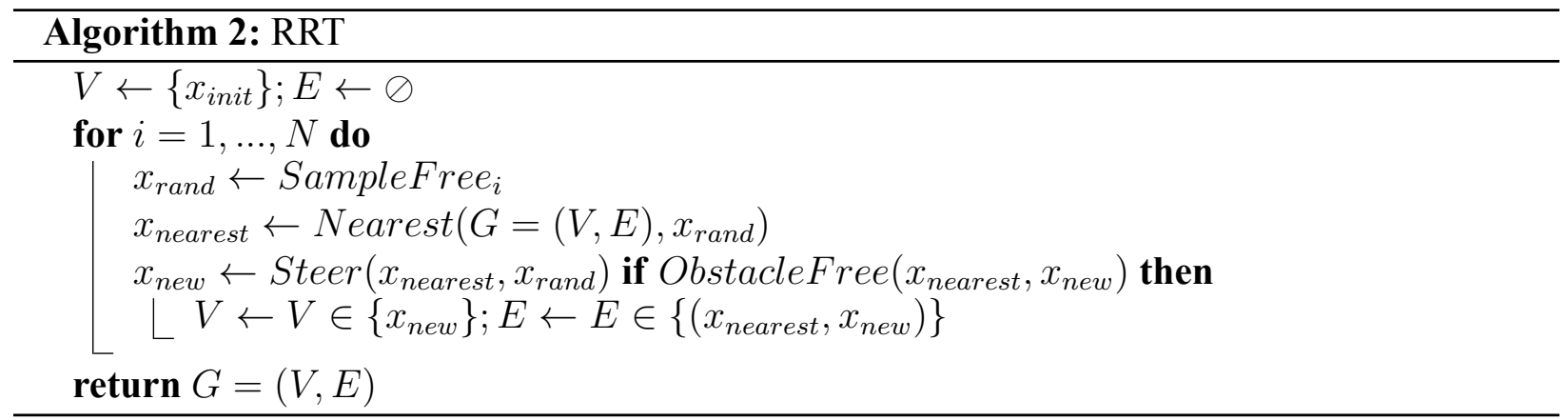


neighbor (which is, initially, the source node until the tree grows to more than one node). This implies that the paths are built at the same time the tree is being constructed, unlike the PRM where the graph is built first, and the paths are found afterwards. A branch of an RRT tree is grown from the closest neighbor in the direction of the random sample and its length is determined by a step size input. The pseudocode is given in Algorithm 2. The user also chooses how many iterations the algorithm runs; the more iterations, the denser the tree is. However, even with a few iterations the tree rapidly explores all the empty space and covers as much of it as possible. A step-by-step illustration of the RRT algorithm is given in Fig. 4.2. It is possible to grow two trees, making it a bidirectional RRT (RRT-Connect), from both the start node and the goal node [Kuffner and RRTConnect, 2000]. In this case, however, there must be a step that tries to connect the two trees as they grow. There could be even more than two trees, called in this case a forest.

In the same manner that PRM evolved, RRT has different variants, including RRT*, that chooses the best neighbor instead of the closest neighbor, with regards to cost. The pseudocode is presented in Algorithm 2 [Carpin, 2006] and is described as follows:

- It selects a random point $x_{\text {rand }}$ among the free samples in the free configuration space.

- It then computes the vertex closest to $x_{\text {rand }}$ that belongs to the tree, $x_{\text {nearest }}$.

- It computes a new vertex $x_{n e w}$ in free space that meets the following requirements: it is within a user-defined distance from $x_{\text {nearest }}$ and requires a steering angle that does not exceed the user-defined maximum steering angle.

- It computes the cost of this new node, i.e. the cost of $x_{\text {nearest }}$ added to the cost of the path from $x_{\text {nearest }}$ to $x_{\text {new }}$.

- It connects $x_{\text {nearest }}$ and $x_{n e w}$ if it is collision free.

At this point of the algortithm, RRT* differs from RRT (so far, all the steps were similar). Indeed, the next step is the rewiring of the tree. Instead of connecting to the nearest parent, it connects to the best parent, meaning that within a certain distance of $x_{n e w}$, the tree is searched for a better parent that would minimize the cost of a path going through $x_{n e w}$. The algorithm is shown in Algorithm 3. RRT* presents several advantages over RRT, primarily asymptotic optimality. Moreover, the cost of the path from the same start point to the same goal is less with RRT* than it is with RRT. The tree cost is also less in RRT*. Even if the computing time is higher, the algorithm achieves a better path (in terms of cost) with RRT*.

\subsubsection{Search algorithms}

Search algorithms can be part of the planning process and many have been developed over the years: Dijkstra, $A^{*}$ and $D^{*}$ are famous examples. Dijkstra developed an algorithm to find the shortest path between two nodes in a graph [Dijkstra et al., 1959], and by extension, it can lead to a tree of shortest paths between a source node and all other nodes (shortest path tree).

In 1968, A* was developed [Hart et al., 1968] and solved for the least-cost path in a graph, if cost is the weight of the graph edges. It is an example of a best-first search algorithm used to solve planning problems (i.e. it expands the most promising node). In the 80 s, research focused on the Mover's problem, that is, a collision-free path for a moving object in a space containing obstacles. An example paper published a solution combining a Voronoi diagram (sampling method, see below) with a best-first search algorithm [Donald, 1987]. This algorithm was further developed to include 

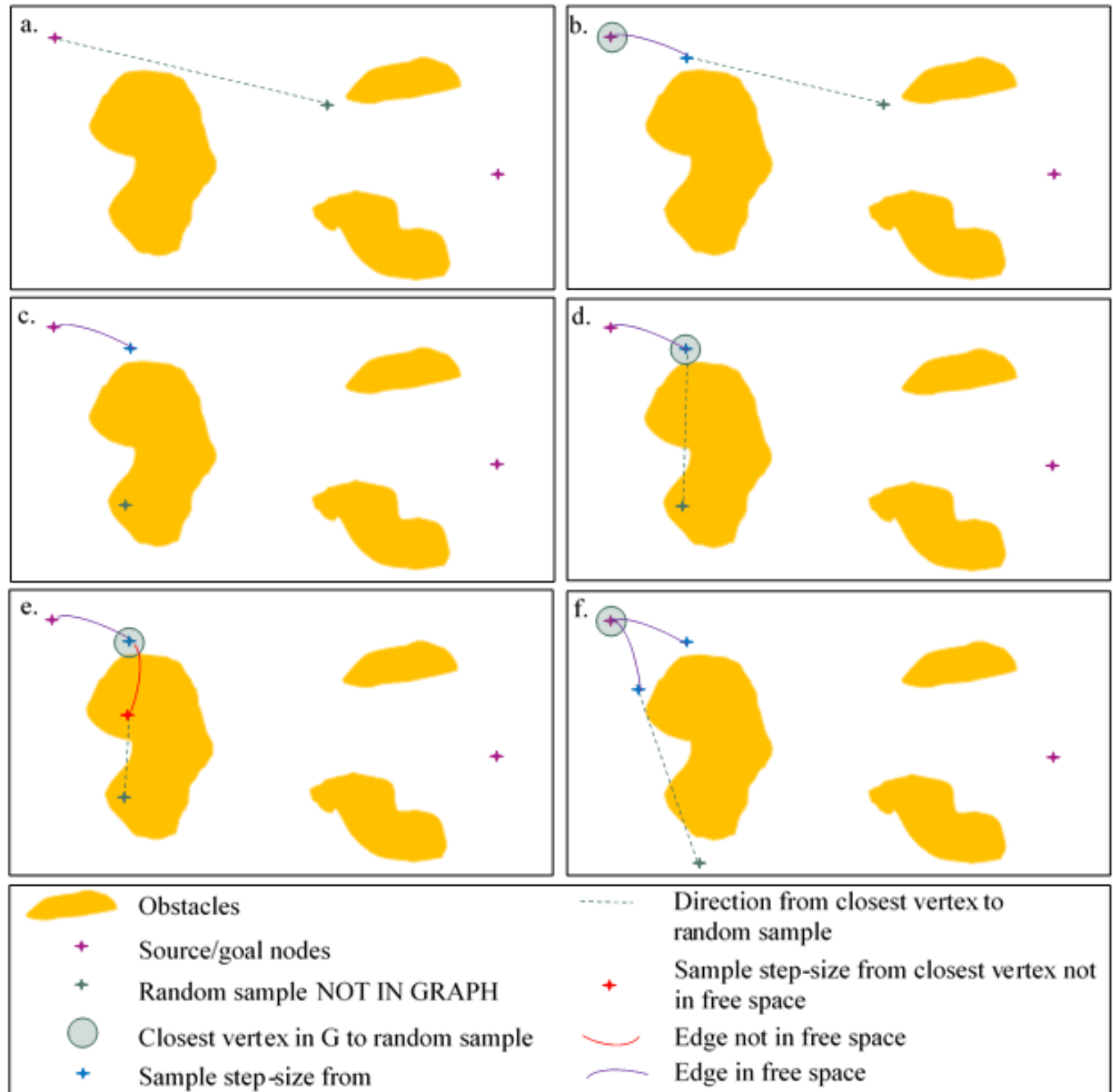
Direction from closest vertex to random sample
+ Sample step-size from closest vertex not in free space
Edge not in free space
Edge in free space

Figure 4.2: Illustration of RRT: (a) random sampling; (b) selection of nearest neighbor, and if in free space, addition of a new node at step-size distance from nearest neighbor with connecting edge; (c) random sampling; (d) Selection of nearest neighbor and collision detection; (e) collision detection; (f) random sampling, selection of nearest neighbor and addition of a node step-size away in the direction of random sample.

variants such as anytime $\mathrm{A}^{*}$, which adds a time limit to the original $\mathrm{A}^{*}$ and also computes suboptimal solutions to the path. Lifelong Planning $A^{*}$ is another alternative to $A^{*}$, which has edge cost decreasing or increasing over time [Koenig and Likhachev, 2002]. The pseudocode for $A^{*}$ is shown in Algorithm 4. D* can also be named as a famous example of a search algorithm [Stentz, 1993] and minimizes the cost of the path to the goal. It can be regarded as an extension of $\mathrm{A}^{*}$ (Focused Dynamic A*) [Stentz et al., 1995]. The cost is user-defined, meaning it could be related 


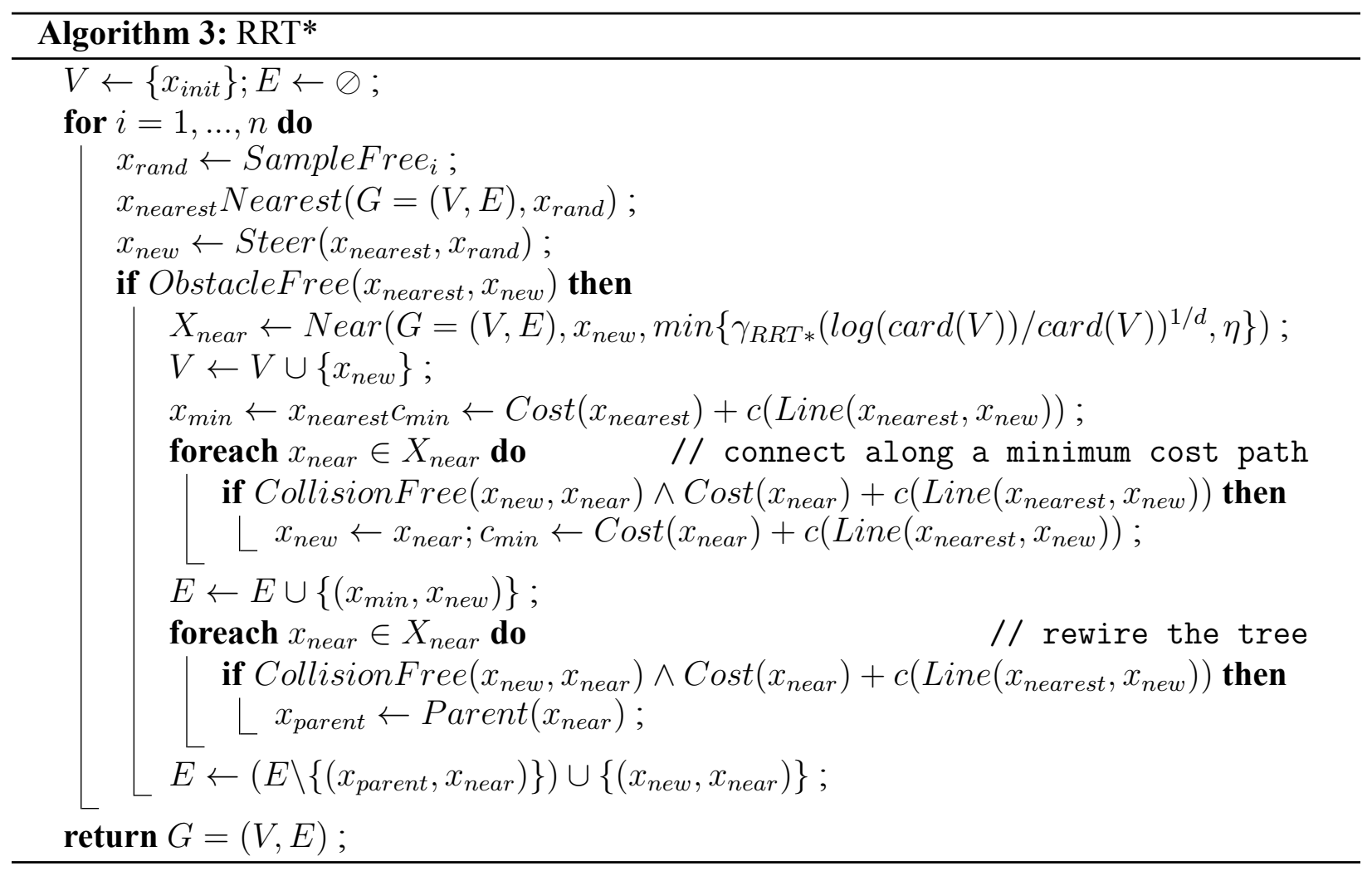

to time (fastest path), safety (most secure path), etc... as in $\mathrm{A}^{*}$, but it also allows for replanning if the configuration space happens to be different than initially assumed, which is a crucial feature of real-world application [Corke, 2011]. Extensions of the D* algorithm include D* lite and field D* among others. $\mathrm{D}^{*}$ lite computes the shortest distances to the goal of each grid cell given a certain gridmap, recalculates them when it runs into an unexpected obstacle and has to change the initial planned path. It can efficiently recompute the shortest path by considering only the cells whose distances to the goal have changed [Koenig and Likhachev, 2002]. Field D* is worth mentioning as it was implemented on board Mars Exploration Rovers (MERs) in 2006 [Carsten et al., 2007]. While the other aforementioned algorithms consider discrete state transitionn field $\mathrm{D}^{*}$, on the other hand, implements paths along continuous headings by using linear interpolation within each grid cell [Ferguson and Stentz, 2005].

\subsubsection{Planning with a budget constraint}

In recent years, the idea of budget was suggested. Certain variables defined by the user could fall under a certain limit for the algorithm to be successful, called a budget. For instance, the rover traverse needs to be performed in a time limited setting (time budget) or the rover cannot drive if it does not have enough energy (energy budget). Planners such as RRT* have been adapted to this new setup, called Rapidly Exploring Information Gathering algorithm (RIG) [Hollinger and Sukhatme, 2014]. Nodes in RIG-graph or RIG-tree represent location, cost and information all together, which makes this planner well suited for exploratory missions like Perseverance. The 


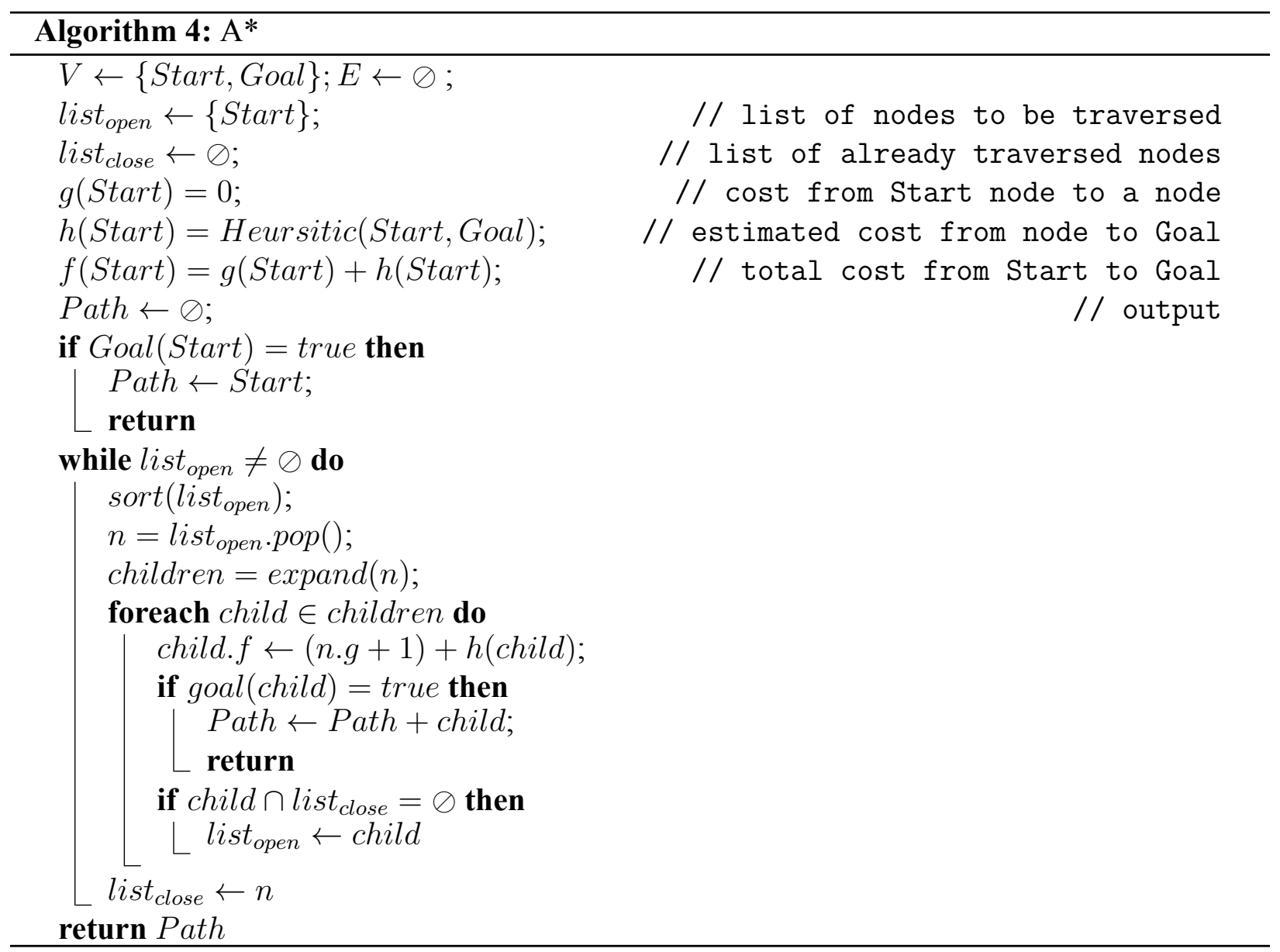

trajectories generated by the algorithm tend to maximize the information metric while being contained within a budget. Some research with budget constraints went even further by proposing an evolution of the path given new information gathered along the drive. The robot then adapts to new costs of edges and replans its path accordingly [Hitz et al., 2017].

\subsubsection{Planning under uncertainties}

Uncertainties can take many forms: missing information about the environment, actuator errors, modeling errors, etc... There are ways to account for such uncertainties during planning, using methods like the Markov Decision Process (MDP) and reinforcement learning, where a behavior is rewarded positively or negatively, depending on what is wanted of the robot.

\subsubsection{Markov Random Field (MRF) and belief propagation}

An approach to path planning worth mentioning utilizes Markov Random Field (MRF) to find the maximum-likelihood field of principal directions, using features detected by the vehicle in its surroundings [Dolgov and Thrun, 2008]. This method was developed more specifically for driving into confusing environments such as parking lots, and the argument could be made that Mars fits the description, with many options of directions to go to available to the rover. MRFs have been 
used for terrain classification applications as well, in support of generic path planning [Häselich et al., 2014].

MRF, also known as Undirected Graphical Models (UGMs), are defined as an undirected graph $\mathcal{G}=\{V, E\}$ where $V$ is the set of nodes $\nu_{i}, i \in[1 \ldots n]$ in the graph $\mathcal{G}$ and $E$ is the set of edges $\varepsilon$ in the graph $\mathcal{G}$. The neighboring nodes of vertex $i$ (i.e., nodes linked to $\nu_{i}$ by an edge) is denoted $\mathcal{N}_{i}$ (often referred to as the Markov blanket of $\nu_{i}$, since in a UGM the Markov blanket is equivalent to the neighborhood). Each node $\nu_{i}$ in the graph $\mathcal{G}$ is associated with a random variable $u_{i}$ that satisfies three properties:

- The pairwise Markov property: if there are no direct edges between node $\nu_{i}$ and $\nu_{j}$ then they are conditionally independent given the rest:

$$
u_{i} \perp u_{j} \mid V \backslash \nu_{i}, \nu_{j} \Longleftrightarrow \mathcal{G}_{i, j}=0
$$

- The local Markov property: the conditional probability at nodes $\nu_{i}$ depends on the neighboring nodes only and are conditionally independent of all the other nodes not in the neighborhood:

$$
u_{i} \mid\left\{u_{j}\right\}_{j \in V \backslash i}=p\left(u_{i} \mid\left\{u_{j}\right\}_{j \in \mathcal{N}_{i}}\right)
$$

- The global Markov property; for three sets of nodes $A, B$ and $C$ in graph $\mathcal{G}$, with set $C$ separating set $A$ from set $B$, then the nodes in $A$ and the nodes in $B$ are conditionally independent given the nodes in $C$. This can be written as:

$$
p\left(u_{A} \perp u_{B} \mid u_{C}\right)
$$

with $\mathrm{C}$ a separating subset of $\mathcal{G}$.

MRFs or UGMs can be utilized for belief propagation frameworks, where inference is used between nodes to propagate a belief of one node to others. Probabilistic inference methods have been suggested for the first time in very recent years [Toussaint, 2007], proposing to implement belief propagation on Bayesian network (from directed graphs, unlike MRFs that come from undirected graphs). One of the main methods for belief propagation is the sum-product message passing algorithm [Kschischang et al., 2001], which, as the name indicates, aims at passing a message from one node to the others along the edges. The message passed from node $\nu_{i}$ to node $\nu_{j}$ in graph $\mathcal{G}$ is the product of all messages received by $\nu_{i}$ from incoming edges, and summed over all possible configurations of node $\nu_{j}$. These $n$ configurations can be represented as an $n \mathrm{x} n$ square matrix, called edge potential matrix. The product of all incoming messages can be illustrated as a factor node in a factor graph, located between variable nodes. Variable nodes $x$ refer to nodes that are associated to random variables (e.g., $x_{i}$, variable associated with node $\nu_{i}$ ) and factor nodes $f$ refer to function nodes that are not shown in classical graphs, but are represented in their factorized counterparts (Fig.4.3). Those factor nodes translate the operation of local functions between variable nodes (in the case of sum-product message passing, the factor node represents the product of local functions, summed over all configurations of the variable). An illustration of a message passing process is shown in Fig.4.4. The equations for the belief propagation are detailed in Eq.4.4, 4.5 and 4.6 [Kschischang et al., 2001], using the nomenclature of Fig.4.4:

$$
\phi\left(x_{i}\right)=\sum_{\{f\}} \mu_{f \rightarrow x_{i}}\left(x_{i}\right)
$$




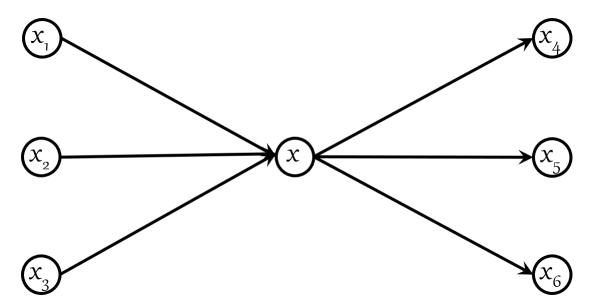

(a) Directed graph.

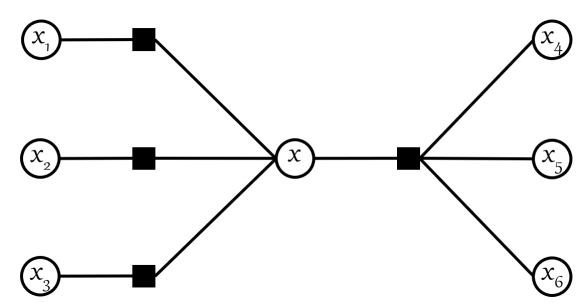

(b) Equivalent factor graph.

Figure 4.3: (a) Directed graph and (b) equivalent factor graph.

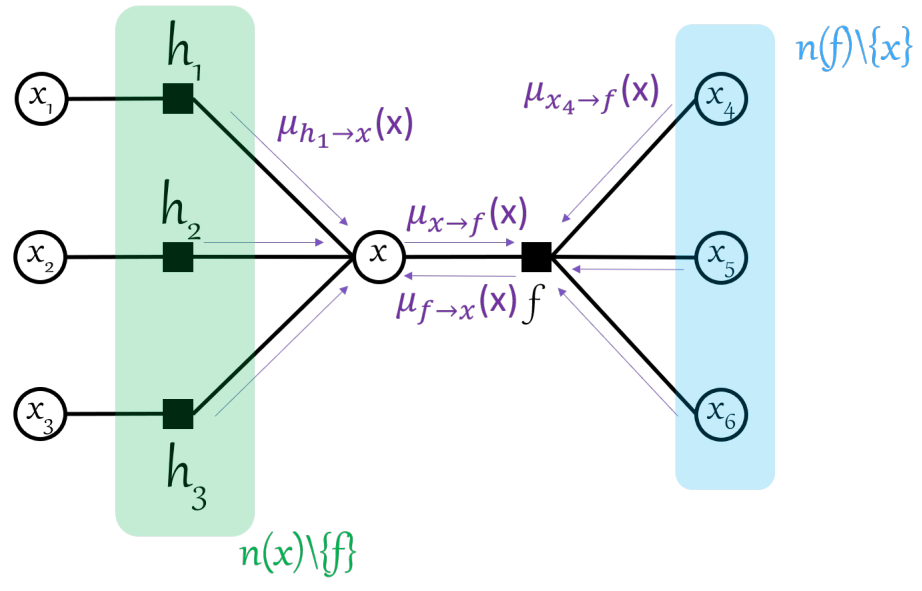

Figure 4.4: Message passing on a couple of nodes.

$$
\begin{gathered}
\mu_{f \rightarrow x_{i}}\left(x_{i}\right)=\sum_{\{x\} \backslash x_{i}}\left(f(n(f)) \prod_{x_{j} \in n(f) \backslash x_{i}} \mu_{x_{j} \rightarrow f}\left(x_{j}\right)\right) \\
\mu_{x_{i} \rightarrow f}\left(x_{i}\right)=\prod_{h \in n\left(x_{i}\right) \backslash f} \mu_{h \rightarrow x_{i}}\left(x_{i}\right)
\end{gathered}
$$

where $\mu_{f \rightarrow x}(x)$ is the message passed from a factor node $f$ to the variable node $x$ and $\mu_{x \rightarrow f}(x)$ is the message passed from a variable node $x$ to a factor node $f$.

\subsubsection{Other approaches}

Inspired by the PRM algorithm, researchers have developed an algorithm called Belief Roadmap (BRM). It uses the same principle as PRM but with a belief graph, meaning it samples belief poses, i.e. estimated poses of the robot. The search part of this algorithm computes a path that optimizes the probability of being at the goal point by incorporating the resulting covariance at the next node [Prentice and Roy, 2009]. PRM also inspired FIRM, the Feedback-based Information Roadmap, that accounts for motion and sensing uncertainties [Agha-Mohammadi et al., 2014]. Using basemap methods directly in belief space does not guarantee nodes are reachable and creates a dependability of edge costs to one another. By utilizing feedback controllers to construct the belief 
graph, FIRM goes around these difficulties and offers a generalized PRM. Another "adaptation" of a common algorithm is RRBT (Rapidly exploring Random Belief Tree), which uses principles of RRT* applied to belief space [Bry and Roy, 2011]. The nodes and edges propagate beliefs and the overall tree tends to minimize uncertainties while avoiding obstacles.

Another approach are Markov Decision Processes. They are an important part of decision making with uncertainties and have been around since at least the fifties [Bellman, 1957]. The process is based on actions and states: at each time step the robot is in a certain state and is moved to the next state by an action. The state transition to the next step depends on the previous step, however the process satisfies the Markov property (i.e., the conditional probability distribution over futures states depends on the current state only, regardless of past events). Moving to a new state generates a reward (or a cost), and such reward function defines the behavior of the model overall [Russell and Santos Jr, 2019]. MDP can be generalized to belief states instead of configuration space, in this case called Partially Observable Markov Decision Process (POMPD). POMDPs are usually applied when the world cannot be observed completely, relying on belief instead of actual states. Belief space is the space of believed states, i.e. the probability distribution over states [Bonet and Geffner, 2000]. It can also be understood as state uncertainty and takes into account the history over previous states. POMDP comes with two curses: the curse of dimensionality and the curse of history. The first comes from the fact that a belief is a probability distribution over all possible states. The second comes from the belief capturing information from a history of actions and observations, therefore growing exponentially over time. Decision making under uncertainties is extremely important to model everyday problems in a realistic way, and methods such as POMDP are well suited to solve these. The advantage of POMDPs is their ability to handle a multitude of uncertainties of different kinds [Aberdeen, 2003] and they can be used for a lot of real problems, such as: machine maintenance, deep-space navigation, machine vision, interplanetary rovers, or even business application such as marketing and network troubleshooting [Cassandra, 1998]. POMDPs cannot, usually, be solved exactly [Kurniawati and Yadav, 2016] and research has thus focused on finding approximate solutions to face this challenge [Aberdeen, 2003]. There are offline approaches that compute the policy over the entire belief space (but they can take a significant amount of time to find a solution [Ross et al., 2008]), and online methods that compute the policy only at time of execution [Paquet et al., 2006, Ross et al., 2008], thus requiring real-time heavy computation. Some authors have suggested a mix of online and offline methods, to take advantage of both methods, [Paquet et al., 2006, Ross et al., 2008]. Path planning is a very common application of POMDPs, as incorporating uncertainties into the planning phase allows for more realistic scenarios for mobile robots. However, among the three main types of uncertainty (environment, sensor and motion), most POMDPs only consider sensor and motion, not environment [Kurniawati et al., 2012]. This type of uncertainty is usually directly treated within the planning method as a modified planning algorithm, such as an extended PRM [Missiuro and Roy, 2006] or RRT [Kewlani et al., 2009]. 


\subsection{Terramechanics}

\subsubsection{Definition}

Another way to assess traversability that can be done in real time is through the study of terramechanics, which is the interaction of a vehicle with a terrain. More specifically, it is the study of how a vehicle responds to driving on a given surface [Muro, 2004]. Several forces and stresses play a role in terramechanics, including pressure of the wheels onto the terrain and normal and shear stresses created by the wheel when driving. These determine a response from the soil such as a deformation and a resistance to sinkage (compaction resistance). It has a variety of applications, ranging from agriculture to military operations and planetary rovers.

\subsubsection{Historical background}

Early work on the mechanics of off-road vehicles can be dated as far back as 1913 in Germany [Bekker and Bekker, 1962]. This field of study has become especially important since World War II [Wong, 2008]. If it was not an independent discipline at the time, it became its own field following the work of Dr. M.G. Bekker, who set the path to modern terramechanics in the 60s [Wong et al., 1989]. Application to planetary surface missions was mentioned as early as 1962 [Bekker and Bekker, 1962] and has been an expanding field for the past two decades. Lunar rovers were used to study traversability of the regolith and identify soil properties [Carrier, 2006].

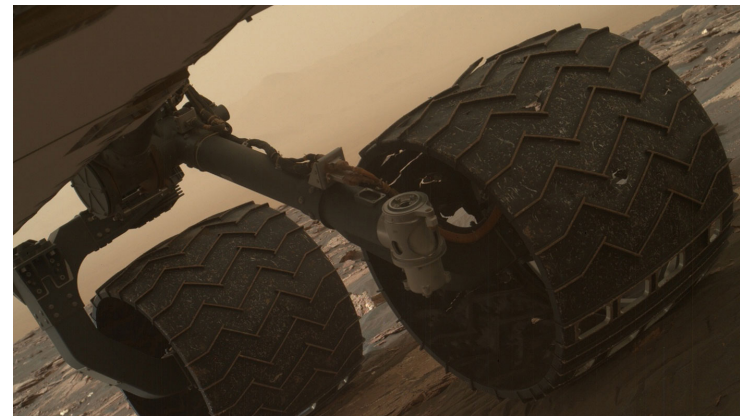

Figure 4.5: Curiosity wheel showing holes after traversing rough terrain with sharp rocks. Credit: NASA JPL.
With the launch of several surface missions back to the Moon and especially to Mars, terramechanics has been a growing focus. After the loss of the rover Spirit at Gusev Crater in 2010, it has become even more important to understand terrain properties and how vehicles interact with the terrain they are driven on. Spirit traversed compacted terrain at the beginning while on Gusev plains, however, upon arriving at the Columbia Hills, it encountered highly deformable sulfate rich soil that made traversability a challenge [Johnson et al., 2015]. Moreover, these soils were covered in basaltic sand, making them hidden on images. The rover underwent high sinkage (up to $10 \mathrm{~cm}$ ), and the failure of the right front wheel actuator made the traverse even worse as Spirit was forced to drag its wheel along. It eventually got embedded in a sand filled crater when the left side of the rover tilted into the crater and the wheel got stuck in sand [Johnson et al., 2015]. Failure to extricate Spirit led to the end of its mission in 2010. Opportunity encountered high wheel sinkage situations at Endeavour Crater when it came across the Purgatory ripple field [Arvidson et al., 2011]. Finally, Curiosity also experienced mobility difficulties with wheel damage (holes and dents) from roving on sharp rocks [Arvidson et al., 2017]. To better understand the mobility and difficulties experienced by the rover, the team turned to terramechanics. Understanding the terrain and its interaction with the wheels may have prevented any damage, and there is a real need 
to understand terramechanics to prevent mobility issues [Arvidson, 2014].

\subsubsection{Terramechanics equations for a driven wheel}

The foundations of terramechanics rely on equations translating the interaction between a driven wheel and the ground. They were derived for a single rigid wheel on a rigid terrain, and the research in the field is based on this set of equations. The derivation is shown in Appendix A (inspired from a previous master's thesis completed at Washington University in St.Louis). Overall, these equations are meant to understand the behavior of a terrain under the pressure of a driving wheel. The main force behind the driving traction is the shear stress in the longitudinal direction $\tau$, a function of the normal stress $\sigma_{n}$ [Agarwal et al., 2019]. These stresses are influenced by the type of wheel and the type of soil. Throughout the history of the field, authors have been working towards better understanding every aspect of the interaction between the wheel and the underlying terrain, and all the changes the soil undergoes when a vehicle drives on it. It is important to understand the pressure created, the shear stresses within the terrain, the displacement of the soil, the resultant sinkage, and how the wheel responds to the terrain as well, through slip and skid. Most of the equations are compiled in Wong's Theory of Ground Vehicles (2008) and is highly inspired by the work by Bekker in 1960 and 1969, in his Introduction to Terrain-Vehicle Systems.

\subsubsection{Rover problem}

Let's now consider a vehicle with multiple wheels; it must develop enough thrust to compensate all the resistances it encounters, especially up slope [Wong, 2008]. The drawbar pull (net force) $F_{d}$ is defined as the difference between the thrust $F$ and the sum of all resistance forces acting on the rover $\Sigma R_{R}$ [Wong, 2008] as shown in Fig. 4.6 (Eq.4.7 and 4.8). The approximation is made that it is in a steady-state operating condition over a drive (rover commanded to drive at constant velocity). In this case, the drawbar pull should approach zero, which means the thrust developed balances the total resistance:

$$
\begin{gathered}
F_{d}=F-\sum R_{R} \\
\sum R_{R}=R_{a}+R_{\nu}+R_{c} \pm w_{t} \sin \theta_{s}
\end{gathered}
$$

$R_{a}$ is the aerodynamic resistance (usually not a significant factor that affects the performance of the rover since it is driving slowly, no more than $35 \mathrm{~m} / \mathrm{hr}$ ), $R$ is the motion resistance, $R_{c}$ is the compaction resistance, $w_{t}$ is the weight of the vehicle (constant, $180 \mathrm{~kg}^{*} 3.75 \mathrm{~m} / \mathrm{s}^{2}=669.6 \mathrm{~N}$ ) and $\theta_{s}$ is the slope angle. When the rover drives up slope the negative sign is used, and when it goes down slope the positive sign is used. The motion resistance is the obstacle resistance that increases when the rover encounters an obstacle. Thus, the main factors that are taken into consideration in the drawbar pull as the vehicle drives are the thrust, the slope angle, the presence of obstacles, and the compaction resistance, i.e., sinkage. The amount of shearing between the vehicle and the terrain will determine slip or skid, thus the thrust, and how the vehicle will respond during its drives. Therefore, the soil parameters, which control the shear stress, are crucial. Note that here rover slip and skid are considered (also called 3D slip and 3D skid), as opposed to wheel slip and skid as 


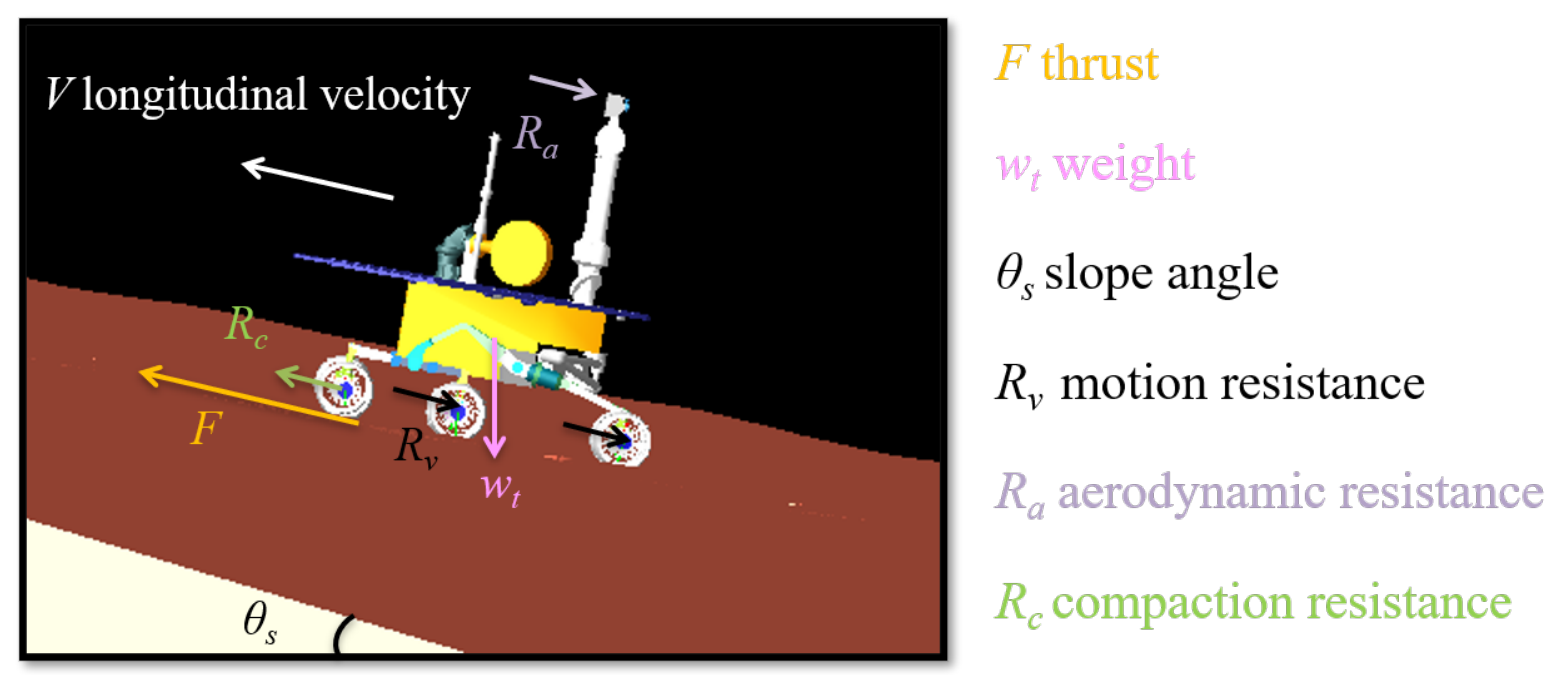

Figure 4.6: Drawbar pull, thrust and resistances for a vehicle driving uphill. Credit: G.Coutrot.

derived in the basic equations of terramechanics. $3 \mathrm{D}$ slip $i_{3 D}$ is defined as follows:

$$
i_{3 D}=100\left(1-\frac{d_{a}}{d_{c}}\right)
$$

where $i_{3 D}$ is slip (when positive), $d_{a}$ is actual distance driven and $d_{c}$ is commanded distance. When $i_{3 D}$ is negative, it actually represents skid $i_{s, 3 D}$.

\subsubsection{Deformable wheels}

\subsection{Exploration rovers: terramechanics and terrain information integrated into path plan- ning under terrain uncertainty}

As said previously, planetary applications of terrain information into planning have been mentioned as early as 1962, and 30 years ago, papers were already publishing research regarding autonomous navigation for a planetary rover with intent to go to Mars [Miller et al., 1989].

Planetary rovers present a different challenge. First, they require a certain autonomy that varies from mission to mission, because they operate far from the human handler, and the command cannot be executed right after being sent to the robot. For example, it takes 8 to 42 minutes to reach Mars. Furthermore, the commands have to go through the DSN for both uplink and downlink, and each happens only once a day [Bajracharya et al., 2008]. One thing is certain, the more advanced the missions get, the more autonomy is needed to complete them. Secondly, terrains on planetary bodies are unknown, therefore driving on them presents some uncertainty. How will the rover behave? Is it safe to drive along the initial planned path or is it necessary to replan? Thirdly, there are computational limits on board a rover even with the advancement of technology. Currently, the Mars rovers rely primarily on images taken on the surface and are limited by how far the camera can see. Scientists and engineers on Earth are able to analyze the images and process the path of the rover for the next few sols. Increased autonomy has been a focus for a long time to allow the rover to take over certain tasks, such as terrain and path assessment. 


\subsubsection{Terramechanics and path planning}

With recent missions to Mars, there has been an increasing interest into terramechanics to better understand Mars terrains, as well as improve the safety of driving. Indeed, terramechanics as applied to planetary vehicles has two outcomes: one, understanding how the rover behaves on a certain terrain improves the safety of driving; and two, the behavior the rover exhibits can give information about the terrain itself, specifically soil properties, through the Reece moduli and Mohr-Coulomb equation (Eq.2.3). As seen in the derivation (Appendix A), terrain parameters and rover variables such as wheel diameter are related by many equations in terramechanics. Therefore, scientists have started using terramechanics as a mean to gain knowledge into planetary surfaces [Arvidson et al., 2014].

Before introducing terramechanics, the terrain component was accounted for during path planning using sensors only [Gat et al., 1990]. However, most sensors do not give an accurate indication of the terrain type, nor do they tell how the rover could behave on it. Terrain topography and vehicle dynamics began to be analyzed as part of the planning process, going beyond simple obstacle avoidance [Shiller and Chen, 1990].

Not until the late $90 \mathrm{~s}$ did terramechanics, as applied to rovers, make an appearance. The modeling of off-road vehicles and their interaction with the environment began to be considered for planetary rovers. A few mentioned planetary exploration as an application of planning for off-road vehicles, but it was not the focus of the papers. Some research was then done specifically with the intent of applying terramechanics to Mars missions, using a physics-based model involving both the rover and the environment [Farritor et al., 1998]. Slip, soil deformations, soil compaction and wheel elastic deformation were integrated into a model used in path planning [Amar and Bidaud, 1997]. Mars was in the spotlight after the Pathfinder mission successfully landed in July 1997 and NASA was planning for other missions to be sent to the red planet, making autonomy a crucial topic (which it still is, considering the increasing complexity of the proposed missions).

Work mostly focused on planning in rough terrain, with some considering the uncertainty of planning [Iagnemma et al., 1999]: the algorithm starts with a regular A* search and an estimate of the terrain roughness to map a path. Then, a physical model of the rover rigorously analyzes the path to determine its feasibility. If necessary, A* replans the path. The algorithm takes into account terrain uncertainty, wheel/ground interaction uncertainty, model uncertainty and path following uncertainty. Numerous papers were published regarding the integration of terramechanics into path planning, following the same scheme: the algorithm plans a first path, then the rover evaluates the terrain based on wheel/ground interaction, and finally the path is recomputed if necessary. This is well illustrated in Ishigami et al. (2007), with an algorithm that uses wheel slip as a method of terrain evaluation. A traversability index has been proposed, combining slope, terrain roughness (irregularity), hardness (e.g. compacted, loose, etc...), and continuity (e.g. cliffs, valleys, etc) [Howard et al., 2001]. Others have suggested to integrate a full dynamic model of the robot into the path planning, comprised of two sub-models: 1) a vehicle sub-model to get a mobility profile, and 2) a terramechanics sub-model to obtain interaction forces on deformable soils. Such model is used to calculate a dynamic mobility index to assess feasible paths to get to a target but could be computationally expensive [Ishigami et al., 2011]. More research was done towards planetary exploration in subsequent years to improve on autonomy. 
Monitoring terrain characteristics has clearly been identified as an important task to be performed to improve effectiveness of a mission. Wheel slip, torque, sinkage and drawbar pull are among the parameters suggested to sense the terrain [Iagnemma et al., 2003]. The rationale behind considering wheel slippage among others is due to regolith covering planetary bodies such as the Moon and Mars. Wheels will slip on such surfaces, as has been proven by Spirit and Opportunity [Arvidson et al., 2011], and it can be measured in multiple ways, such as using a simple IMU (Inertial Measurement Unit) or proprioceptive sensors [Kilic et al., 2019]. One suggested method uses onboard cameras to classify terrains remotely and predict slip. The terrains are divided into three categories: traversable, not traversable, and uncertain, and $\mathrm{D}^{*}$ is used to compute the optimal path. This system has been specifically intended for planetary rovers after seeing the difficulties encountered on Mars [Helmick et al., 2008]. However, the major drawback of most slip prediction methods is that they are performed online, requiring significant computational resources, and are often limited to line-of-sight estimations. To remedy the issue, a software has been developed capable of modeling drives on a user-defined terrain, called ARTEMIS (Adams -based Rover Terramechanics and Mobility Interaction Simulator) that simulates traverses for Opportunity and Curiosity [Zhou et al., 2014]. The software has been validated with the single wheel experiment at MIT [Iagnemma et al., 2004, Senatore et al., 2014], as well as test in the JPL Mars Yard and field experiments in the Mojave Desert, and is currently used to simulate drives for the Curiosity rover [Zhou et al., 2017]. However, similarly to most slip prediction methods, this is also constrained to limited distances on ground assessed via ground images, since orbital imagery is usually not enough to characterize a terrain, as it does not capture current conditions at the surface [Gaines et al., 2016]. To address this issue and in an effort to render missions more autonomous, NASA is conducting research on terrain classification and traversability map with SPOC [Rothrock et al., 2016] and MTTT [Ono et al., 2016], respectively. Both were used to assess driving at the Perseverance landing site candidates and could become essential to studying terramechanics for planetary applications. The main differences between ARTEMIS and MTTT is the modeling of the rover itself in ARTEMIS and the larger scale of MTTT.

\subsubsection{Path planning under terrain uncertainty}

Path planning in real environment presents three main types of uncertainty: motion, sensing, and environment [Kurniawati et al., 2012]. Most of the time, environmental uncertainty is treated within the planning method as a modified planning algorithm, such as an extended PRM [Missiuro and Roy, 2006]. This approach suggests changing the sampling method such that it is biased towards region of higher certainty, leading to better paths for a small number of nodes. However, in the case of equal terrain uncertainty across the map, this method might not provide good results. Moreover, the authors specify that it cannot be applied to higher dimensions (i.e., 3D). Another approach that also integrates terrain uncertainty into path planning is a modified RRT [Kewlani et al., 2009], which uses a mobility-based heuristic to compute paths. More specifically, it computes a new heuristic based on the probability of rollover, and therefore takes into account slope uncertainty. However, this method requires online computation, which can be limited on board a planetary rover.

Research has been done in path planning for planetary rovers to increase their autonomy, for 
example by gathering valuable scientific information autonomously and using it to navigate [Girdhar and Dudek, 2016]. Even though it is a path planning method, it is geared towards scientific exploration more than towards planning a path from A to B. This concept closely resembles a map update, where the rover gathers information about its environment online to update its knowledge and plan its traverse accordingly. This has been demonstrated to work for local planning in an unknown, off-road environment [Fankhauser et al., 2018], and for planning in hazardous environments containing radioactive materials [Mascarich et al., 2019]. However, the mapping and planning phases are both performed online, which might not be suitable for a planetary rover with limited onboard resources and time to perform computations. Another recent article also proposed

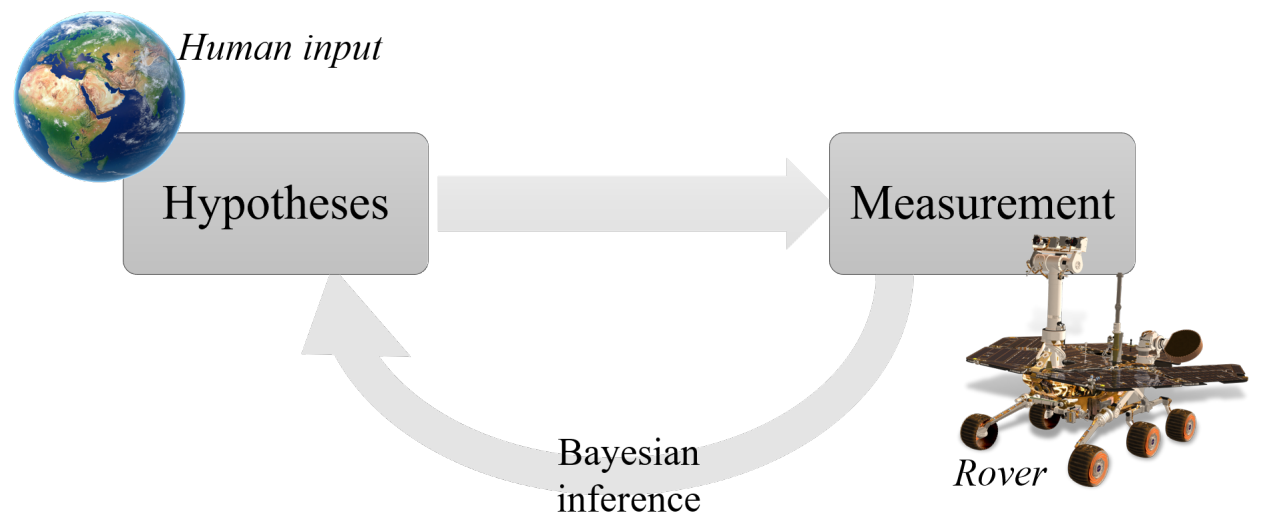

Figure 4.7: Principle of a spectrometer as a guide to exploration: scientists create a hypothetical geology map that can be updated as the rover gains more scientific information about the terrain [Candela et al., 2017].

to integrate the environment directly into the path planning, where the initial map is a belief map of geological units assessed by scientists (hypothesis). The rover is equipped with a spectrometer, and updates its route as it is gaining information about the terrain (proof) through Bayesian inference [Candela et al., 2017]. The principle is illustrated in Fig. 4.7. This paper was inspired by previous work on incorporating Bayesian processes to make planetary rovers more independent in their scientific exploration [Arora et al., 2016]. However, this method might not be easy to implement for a sample return rover, as the systematic use of a spectrometer would be time consuming (about three hours each time [Gellert et al., 2009]) and could be computationally demanding, especially for a mission whose main purpose is not to conduct science experiments in real time. Using a different instrument could potentially alleviate this difficulty, provided the hardware is easy to implement and deploy (e.g., cone penetrometer or shear vane [Rahmatian and Metzger, 2010], detailed in the next subsection).

\subsubsection{Geotechnical properties of soils that can support planning}

Approaches to terrain uncertainty presented in previous sections were focused on integrating geology into planning. As mentioned before, the use of instruments to investigate the environment has been explored and this section details such research performed on geotechnical ex- 
periments for planetary missions, mostly applied to the Moon. Geotechnics as a means to assess traversability on other worlds was closely studied in the 70s, with the Lunakhod rovers and the Apollo missions. Direct methods to measure geotechnical properties are impractical to implement on other planets (see section 2.2.2); therefore, indirect methods were considered. Experiments were sent to the Moon on manned and unmanned missions to retrieve properties of the regolith [Zacny et al., 2010]. Indeed, the Apollo astronauts were equipped with a cone penetrometer to measure bulk density and angle of internal friction [Costes et al., 1971]. A cone vane penetrometer (a cone penetrometer with shear vanes) was carried on the Lunakhod rover to take measurements of the bearing capacity of the Lunar soil [Costes et al., 1971]. On Mars, the rover wheels were used to derive the intrinsic parameters $c$ and $\phi$ to better understand the soil [Sullivan et al., 2011]. For future missions, similar instruments to what was flown to the Moon by the Soviet Union or the United States have been proposed, with improved capabilities, to support more extensive geotechnical studies on other planets [Chhaniyara et al., 2012]. Such examples include a percussive dynamic cone penetrometer [Zacny et al., 2010], a low velocity penetrometer [Seweryn et al., 2014] or a Geovane [Rahmatian and Metzger, 2010]. 


\section{CHAPTER 5 Technical Approach}

\subsection{Introduction}

As stated in Chapter 3, this research can be divided into three parts: orbital analysis (global map), path planning (pre-planning and replanning), and terrain assessment (in-situ analysis). This Chapter will detail the three components, each one based on the previous. The first step is to obtain the map of traversability information that will be used for path planning. The second step is the path planning map at local scale (up to $800 \mathrm{~m}$ ) along the traverse. Computing the path involves choosing a planer with options for replanning in case the initial path is no longer the fastest and safest one, so that the rover completes its traverse efficiently and safely. This step also includes a map update algorithm to keep the most up-to-date information about the environment given by the rover as it drives and a method to monitor the terrain to verify the information given by the map provided to the rover before driving. And finally, the third step addresses in-situ terrain analysis to support planning. To avoid further mobility problems that could potentially lead to aborting the mission, the rover should be equipped with onboard capabilities to assess complicated situations and make the appropriate decision regarding its path. This step should be considered an emergency case that should be avoided, but is necessary to guarantee safety of the rover when everything else (orbital analysis, terrain monitoring and map update) is not sufficient to safely assess the traverse. All three steps are detailed in this Chapter and results are presented in Chapter 6.

\subsection{Orbital traversability map: slip and velocity prediction}

The orbital slip prediction was done in collaboration with Dr. Ono at NASA JPL.

\subsubsection{Problem statement}

\subsubsection{Objectives}

The goal is to get traversability information (slip and velocity) at the landing site that could support ground assessment of the terrain. More specifically, the objective is to obtain an estimation of expected slip and a map of expected drive velocity over any given area using only orbital data.

\subsubsection{Information}

Information available for this research are orbital data and ground information. In particular, coverage at previous landing sites (e.g., Gusev Crater or Meridiani Planum) gives topographic information about the area, as well as thermal (e.g., thermal inertia). Terrain types and traversability classes from MTTT are also available (see subsection 2.2.1.3), and rock abundance has been analyzed at previous landing sites. Similarly, slip checks performed by rovers are available for areas visited by previous surface missions. 


\subsubsection{Constraints}

The constraints on this research are mostly on the data: for example, the available data have varying resolutions, sometimes as low as $100 \mathrm{~m} /$ pixels $(p x)$ (e.g., thermal inertia [Fergason et al., 2006b]). Moreover, not all areas on Mars have orbital and ground (e.g., slip checks) coverage, limiting the analysis to rover landing sites. And, even when available, the coverage might not be complete, as is the case with the DTM or terrain type map over Opportunity's traverse.

\subsubsection{Assumptions}

Several assumptions can be listed, including: the fact that the data needed for slip and drive speed predictions are available; that SPOC performance (i.e., the uncertainty related to terrain type prediction) as described in Rothrock et al. (2016) for Gusev Crater is the same at other locations on Mars; and that only the terrain types data contain uncertainty, while the other data sets do not.

\subsubsection{Challenges}

The main challenge is to use data with different resolutions and overlap the sets over studied areas. Another challenge is to propose a method that relies solely on orbital analysis, in order to apply this work to more areas and better support the mission.

\subsubsection{Contributions}

While this section focuses on preparing a map of the landing site containing both velocity and slip information, the contributions come from the slip prediction method. It has indeed been fully developed at WVUIRL with the collaboration of JPL, whereas the process to obtain expected drive speed is the product of NASA.

The contributions of the research on slip prediction are the following:

- To propose a method that allows for direct prediction of slip using orbital data only.

- To provide a means of predicting slip that can support ground terrain assessment.

- To give traversability information over extended distances, as long as the orbital coverage is available, which means that a path can be analyzed with greater certainty prior to the mission landing.

\subsubsection{Orbital data sets}

\subsubsection{Terrain types and traversability classes}

Terrain types have been presented in subsection 2.2.1.2. They are based on HiRISE images that have a resolution of $25 \mathrm{~cm} / p x$, which implies that the terrain type maps share the same resolution. Terrain types can be grouped into five traversability classes, as explained in subsection 2.2.1.3.

To obtain a prediction of rover velocity along the traverse, a map of terrain is needed. To simplify the process, given that only terrain classes are required for velocity prediction according to the MTTT output shown in Fig.2.3, the five classes were mapped without taking individual types into account. The traversability class map was derived from the DTM, and compared against HiRISE images: the untraversable class was matched with craters, and sandy terrains were matched with ejecta blankets and dunes or ripples when they could be detected. Rough terrain was built using grey threshold values from the DTM and the rest of the map was set to benign terrain by default. Therefore, classes 1 and 2 (benign and rough) do not correspond to actual geological units, unlike 


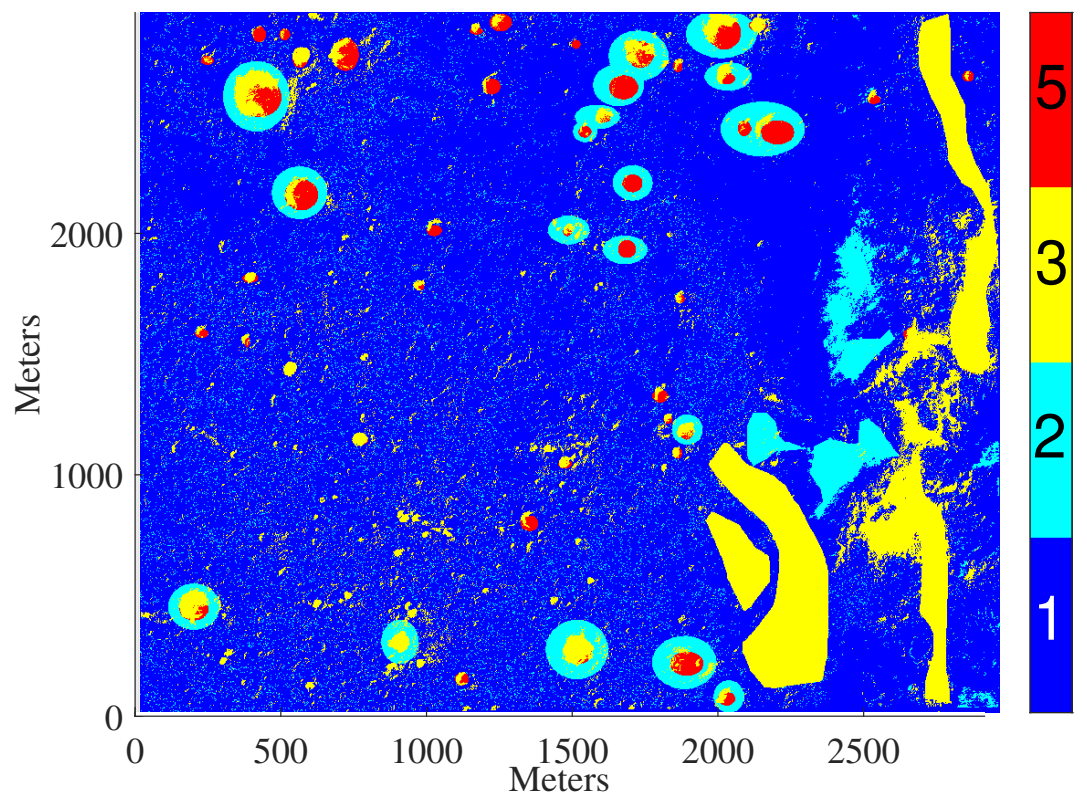

Figure 5.1: Manually interpreted map of traversability classes [Hedrick et al., 2020] (comprised of several terrain types each as shown in Ono et al. (2018)).

classes 3 and 5. The end product has a resolution of $1 \mathrm{~m} / \mathrm{px}$, similar to that of the basemap DTM and an example over Gusev Crater is shown in Fig.5.1. Due to class 1 and 2 being built with grey scale values, they presented with a lot of noise in the southwest part of the map where the values are close to the cutoff. While geological data would show extended area of one or the other, this manually interpreted map shows alternating pixels of one with the other. The north and east parts of the map are less affected by the issue, with values further from the cutoff.

\subsubsection{Cumulative Fractional Area (CFA)}

CFA maps have a resolution of $1 \mathrm{~m} / \mathrm{px}$. Similar to what is presented in the previous paragraph, a map of rock abundance is necessary to the analysis of speed prediction.

To build the rock abundance map, CFA was divided into three ranges (low or $1,<7 \%$, medium or 2 , between $7 \%$ and $15 \%$, and high or $3,>15 \%$ ), which is what is needed for velocity prediction as shown in Fig.2.3. The manually interpreted CFA map was built based on visible craters, assuming that rocks are more abundant around them (high CFA, corresponding to the ejecta blanket), a little less inside them (medium CFA) and a lot less abundant everywhere else (low CFA) [Collins et al., 2012]. The DTM was once more used to build this CFA map, as craters are easily visible in topographic information. The end product has therefore a resolution of $1 \mathrm{~m} / \mathrm{p}$, similar to that of the original data set and DTM. An example over Gusev Crater is shown in Fig.5.2.

\subsubsection{Slope}

Slope also plays a significant role in traversability. The Mars Exploration Rovers (MERs) avoided slope greater than $30^{\circ}$ due to potential sliding on steeper slope [Biesiadecki et al., 2006]. Testing has been conducted to understand traversability on different slopes for Spirit and Opportu- 


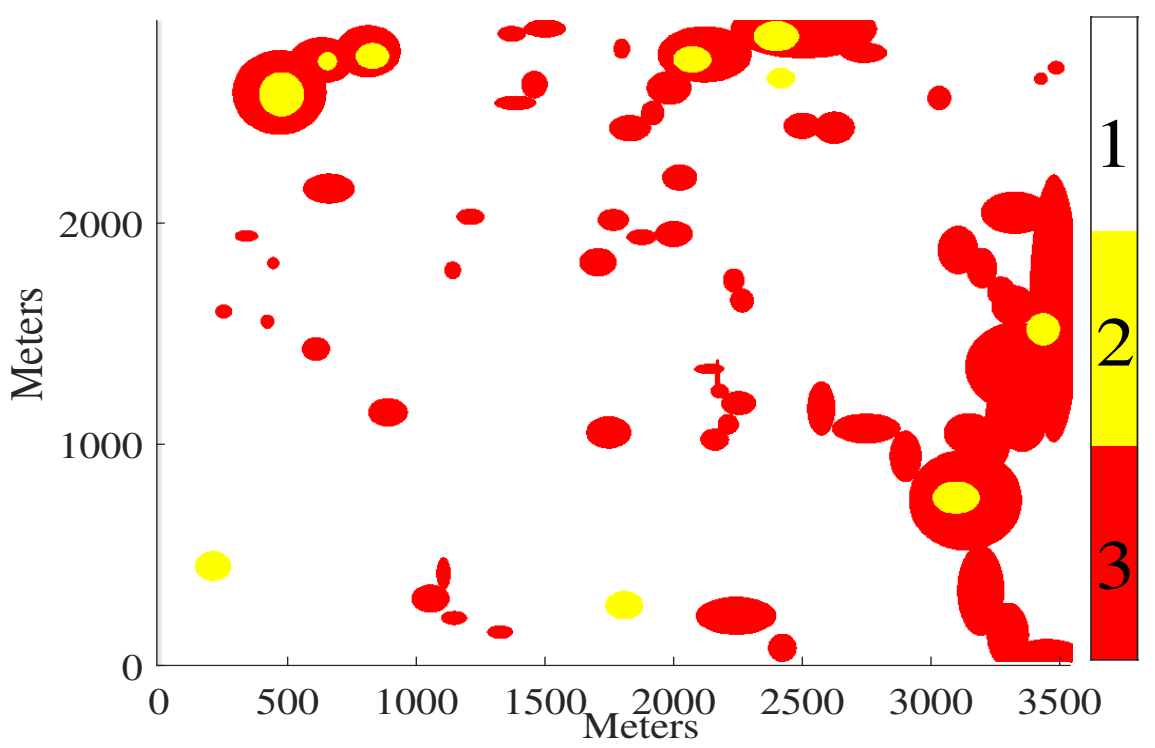

Figure 5.2: Manually interpreted map of CFA ranges: 1 - low rock abundance $(<7 \%), 2$ - medium rock abundance (between $7 \%$ and $15 \%)$ and 3 - high rock abundance $(>15 \%)$ [Hedrick et al., 2020].

nity [Lindemann and Voorhees, 2005] and Curiosity [Heverly et al., 2013]. Slope is derived from Digital Terrain Models (DTMs) over selected areas, which have a resolution of $1 \mathrm{~m} / \mathrm{px}$. It is obtained from the gradient of the height from the DTM times the heading angle in the rover's direction as shown in Eq.(5.1).

$$
S=\left(\begin{array}{l}
\frac{\partial h}{\partial x} \\
\frac{\partial h}{\partial y}
\end{array}\right) \cdot\left(\begin{array}{l}
\cos \theta \\
\sin \theta
\end{array}\right)
$$

Where $h$ is the height given by the DTM, $(x, y)$ are the coordinates of the location for which slope is calculated (pixel of 1 square meter), and $\theta$ is the heading angle, given by Eq.(5.2)

$$
\theta=\operatorname{atan} \frac{x}{y}
$$

The slope is calculated at the $(x, y)$ location by deriving the gradient for the average height over 9 pixels (the pixel $(x, y)$ and the 8 surrounding pixels).

It should be noted that for the velocity study, as shown in Fig.2.3, only five ranges of slope are needed: $\left\langle 10^{\circ}\right.$, between $10^{\circ}$ and $15^{\circ}$, between $15^{\circ}$ and $10^{\circ}$, between $20^{\circ}$ and $25^{\circ}$, and $>25^{\circ}$ [Ono et al., 2018]. The topography of Gusev Crater and the Columbia Hills is shown as an example in Fig.5.3.

\subsubsection{Thermal inertia}

Thermal inertia is derived from the Mars Odyssey Thermal Emission Imaging System (THEMIS) nighttime temperatures [Fergason et al., 2006b]. Thermal inertia depends on several factors including particle size, degree of induration, rock abundance, and exposure of bedrock at the subsurface (within a few centimeters of the surface). It translates the ability of a material to store heat during 


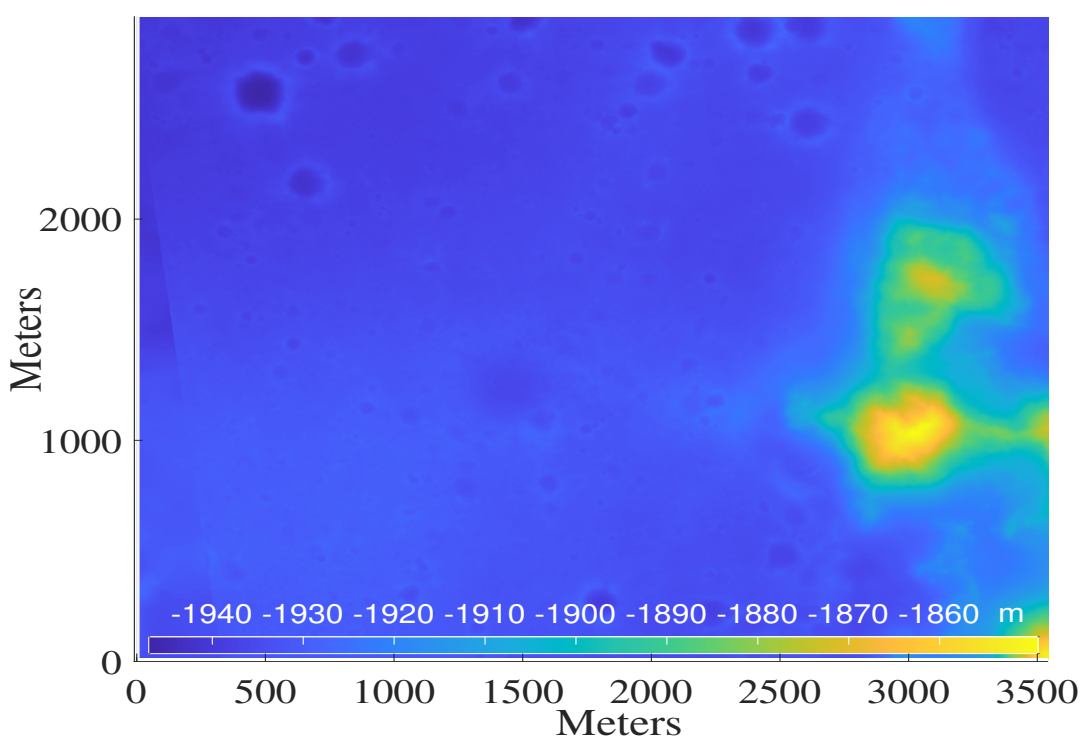

Figure 5.3: Digital Terrain Model (DTM) of the Columbia Hills. Credit:NASA/Washington University in St. Louis.

the day and release it at night [Putzig et al., 2005]. The mathematical definition is as follow:

$$
I \equiv \sqrt{k \rho c}
$$

Where $I$ is the thermal inertia in Thermal Inertia Units $\left(T I U\right.$, with $1 T I U=1 \mathrm{Jm}^{2} \mathrm{~K}^{-1} \mathrm{~s}^{-1 / 2}$. [Putzig

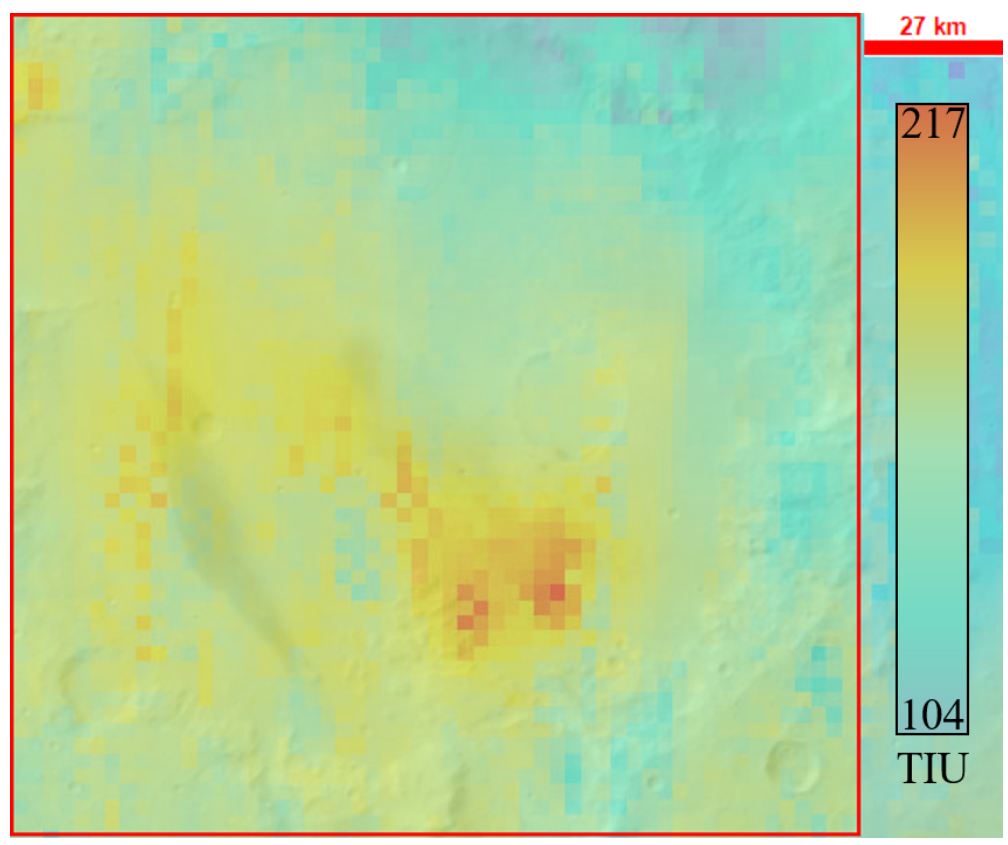

Figure 5.4: Thermal inertia map over Gusev Crater. Values are in Thermal Inertia Unit. Credit: USGS. et al., 2005]), $k$ is the bulk thermal conductivity, $\rho$ is the bulk density and $c$ is the specific heat 
of the surface layer. Usually, the surface layer considered is up to a few centimeters below the surface [Putzig et al., 2005], called skin depth. Thermal inertia can bring a lot of information crucial to terrain classification. In general, high thermal inertia translates into indurated material such as bedrock, which implies that the rover would have less difficulty driving on such terrain. Low thermal inertia is associated with loose material such as sand, which is more challenging to traverse [Cunningham, 2017]. Fig.5.4 shows an example of thermal inertia data over the Columbia Hill, Mars. Thermal inertia maps have a resolution of $100 \mathrm{~m} / \mathrm{px}$.

\subsubsection{Velocity prediction}

\subsubsection{Velocity categories}

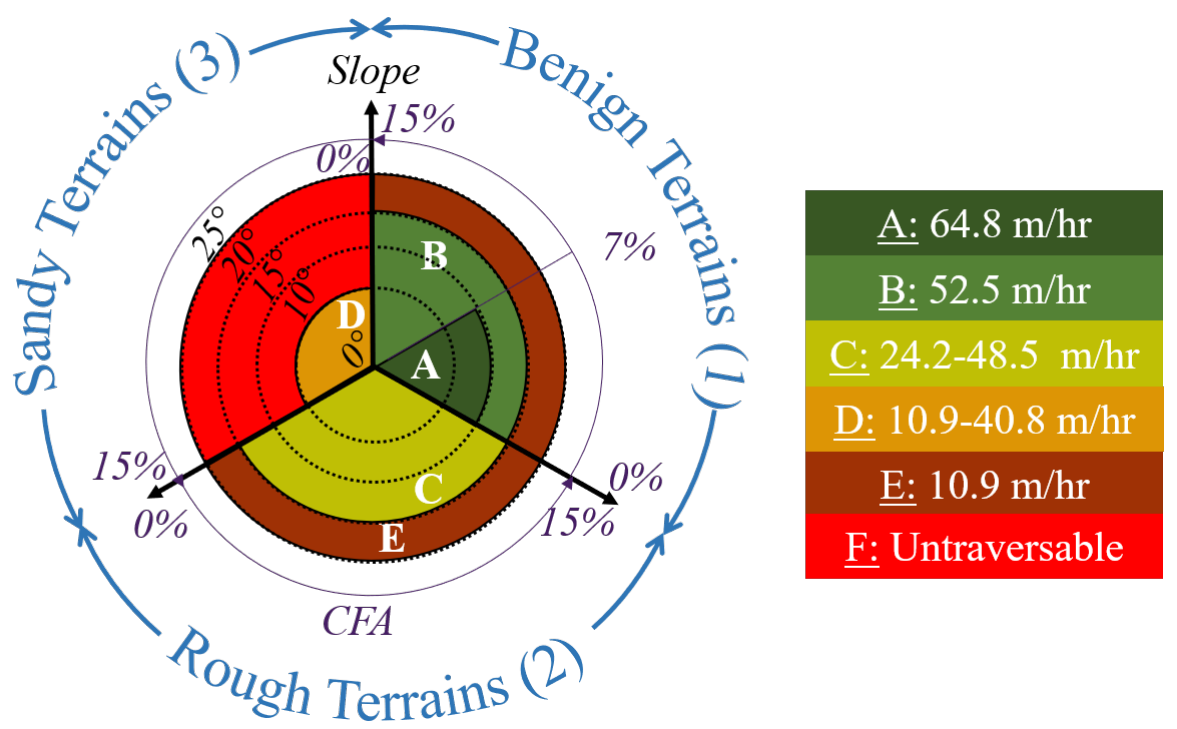

Figure 5.5: Velocity categories [Hedrick et al., 2020]: A - $64.8 \mathrm{~m} / \mathrm{hr}$; B - 52.5m/hr; C - 24.2-48.5m/h; D - 10.9-40.8 $\mathrm{m} / \mathrm{hr}$; E - $10.9 \mathrm{~m} / \mathrm{hr}$; and F - untraversable ( $>15 \% \mathrm{CFA}$ or $>25^{\circ}$ slope, not shown).

According to Fig.2.3, it is possible to predict the rover speed by overlapping three geological data sets: traversability classes from Ono et al. (2018), slope, and CFA [Golombek and Rapp, 1997]. As mentioned before, terrains can be categorized into five traversability classes: benign, rough, sand, no-Autonav and untraversable [Ono et al., 2018]. However, at Gusev Crater, the no-Autonav class (class 4 ) is absent, simplifying the problem to only 4 classes [Rothrock et al., 2016]. Within each class, for given values of slope and CFA, an expected speed of the rover is calculated [Ono et al., 2018]. Therefore, by overlapping the CFA, DTM and traversability class maps over each $1 \times 1 \mathrm{~m}^{2}$ cell, it is possible to estimate an expected speed of the vehicle across each meter of the map. Figure 5.5 shows the categories of speed given traversability class, CFA and slope, labeled A to F.

\subsubsection{Map of velocity categories}

This map is built over an area potentially covering the equivalent of the journey from landing to samples (Spirit's traverse was taken as a reference in Fig.6.1a. To build the map, CFA, traversability classes and slope information are needed, but only the DTM is public information. New maps were therefore visually interpreted and labeled using HiRISE images and DTM over the 
chosen area, as described in subsection 5.2.2. Even if additional uncertainty could be present in the traversability class map due to the fact that the map had to be manually labeled, it is assumed that the data set provides a reasonable approximation of the Mars conditions for evaluating the proposed planning algorithms, and that the uncertainty from the classifier used in Rothrock et al. (2016) can be applied to this work.

\subsubsection{Slip prediction}

This analysis was performed using actual data sets via a limited release by JPL.

To predict slip, both Endeavour Crater and Gusev Crater were considered as potential candidates given the availability of ground truth at these locations (i.e., slip checks). However, for Endeavour Crater, terrain types and CFA were not available, and only public data such as slope and thermal inertia had been obtained. The first step is to run a Principal Component Analysis (PCA) to identify the most useful data sets for slip prediction.

\subsubsection{Principal Component Analysis (PCA)}

Table 5.1: Principal Component Analysis (PCA) results showing PCs (Principal Components) 1 to 4 with the contribution of each variable (in \%).

\begin{tabular}{|c||c|c|c|c|}
\hline & PC1 & PC2 & PC3 & PC4 \\
\hline \hline Variance explained & $\mathbf{4 6 . 5 1 \%}$ & $\mathbf{2 8 . 2 1 \%}$ & $\mathbf{1 4 . 8 3 \%}$ & $\mathbf{1 0 . 4 6 \%}$ \\
\hline \hline Thermal inertia & $6.250 \%$ & $65.62 \%$ & $14.78 \%$ & $13.35 \%$ \\
\hline Slope & $19.82 \%$ & $30.70 \%$ & $44.60 \%$ & $48.80 \%$ \\
\hline Terrain types & $34.94 \%$ & $0.9800 \%$ & $40.55 \%$ & $23.53 \%$ \\
\hline CFA & $38.99 \%$ & $2.710 \%$ & $0.07450 \%$ & $58.23 \%$ \\
\hline
\end{tabular}

A Principal Component Analysis (PCA) on all four variables (thermal inertia, slope, terrain types and CFA), centered and standardized, is performed. It revealed that the first three principal components (PCs) contribute to $89.54 \%$ of the data, almost $90 \%$, and the first two alone contributes to $74.71 \%$ of the data, almost $75 \%$. The biplot in Fig.5.6 shows that if terrain types and CFA contribute the most to PC1, slope and thermal inertia contribute the most to PC2 and most importantly, to the total explained variance by dimension one and two, at $53.70 \%$ and $39.23 \%$ respectively (Fig.5.6). Thermal inertia and slope are thus retained as the main contributing variables and the needed predictors for slip analysis.

\subsubsection{Classifier training to predict slip \\ 5.2.4.2.1 Data points available}

The data are taken from both the Opportunity and Spirit rovers, at Meridiani Planum and Gusev Crater, respectively. Slip was recorded only when Visual Odometry (VO) was enabled (referred to as "slip checks") [Maimone et al., 2007]. However, VO was not always an option since it did not allow for fast speed [Biesiadecki et al., 2007], and consequently, few data points were available for this study, as shown in Fig. 5.7. 2073 data points were collected for Spirit (MER A, Fig.5.7b) and 3250 for Opportunity (MER B, Fig.5.7a). Even though more slip checks were performed at Meridiani, processed DTM coverage is not available everywhere which limits the 


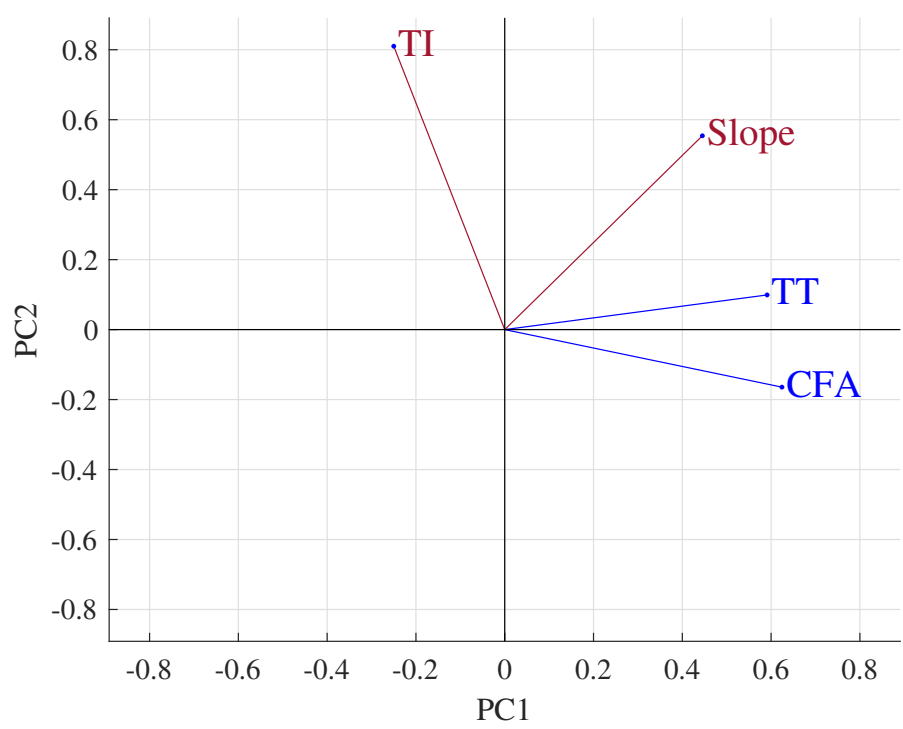

Figure 5.6: Biplot showing the contribution of each variable to the first two PCs that explain $75 \%$ of the data. TI: thermal inertia; TT: terrain types; CFA: cumulative fractional area.

number of points available for use in this research.

\subsection{Data processing}

Slope has been identified for a long time as a factor generating slip experienced by rovers [Bouguelia et al., 2017], but the role of thermal inertia was not as clearly defined until recently [Cunningham, 2017]. It is related to terrain properties as explained in section 5.2.2 and is important for predicting slip, as demonstrated by the PCA analysis in section 5.2.4.1.

The slope and thermal data are combined to train a classifier to predict ranges of slip: lower than $30 \%$ or higher than $30 \%$. This threshold was taken from a similar classification used in Bougelia et al. (2017) with classes medium slip $(30-60 \%)$ and high slip $(>60 \%)$ combined into one category. While their work focuses on tracking ranges of slip from ground data and rover behavior, this research aims at producing a classifier that will allow slip prediction exclusively from orbit. In order to obtain the value for slope and thermal inertia at the location of the slip check, the raw data are imported into Matlab ${ }^{\circledR}$ to be georeferenced. The longitude and latitude for each cell are computed by referencing the center of the cell to Mars coordinates (Mars Equidistant Cylindrical projection [Rosiek et al., 2003]). The next step consists of locating the slip checks, as the data provided are referenced with a time stamp (spacecraft clock or sclk) for each VO measurement. To derive coordinates from the time stamp, the rover position is used, since it is already associated with a sclk. It is first converted from site frame, a local system of coordinates [Pyrzak et al., 2006], to longitude and latitude. Then a shape-preserving piecewise cubic interpolation method is applied to obtain longitudes and latitudes at which slip checks were performed. Finally, once the locations of each slip check are computed, the values of thermal inertia at these locations are obtained by simply matching their locations to those of the slip checks. The same matching is performed on the DTM, with the additional step of averaging the height at these locations with the eight surrounding 


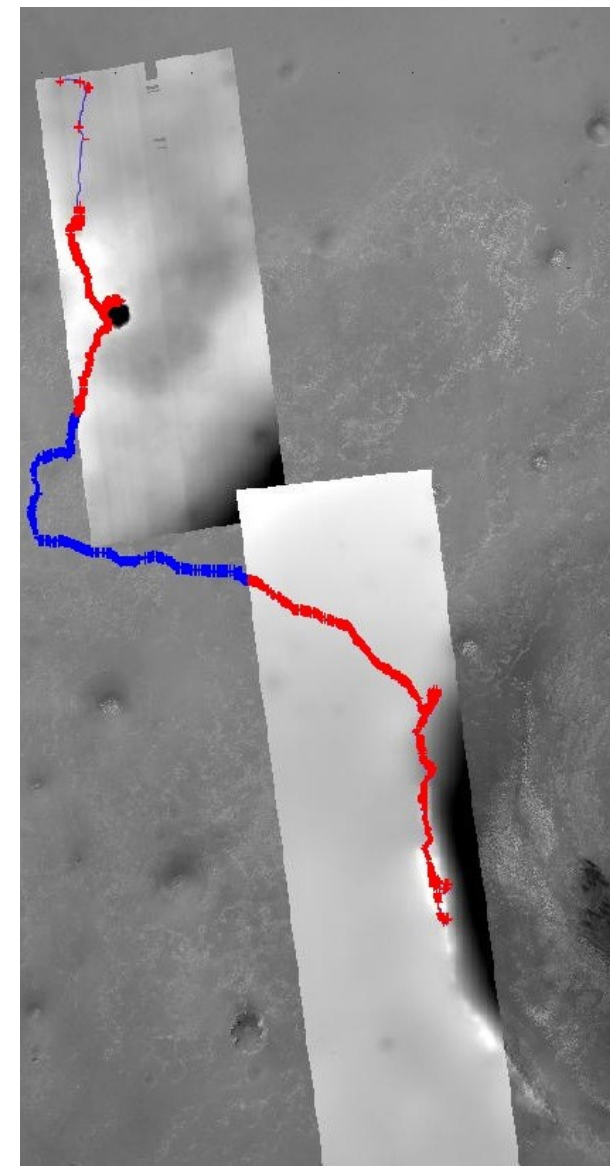

(a) MERB traverse (total length: $45.16 \mathrm{~km}$ ) and slip checks.

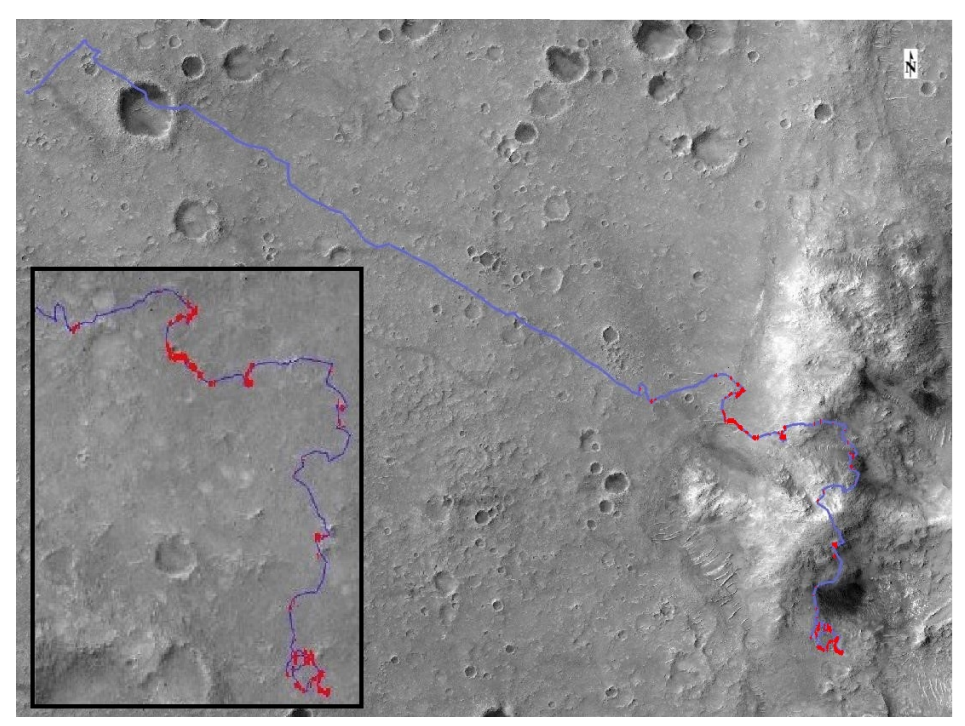

(b) MERA traverse (total length: $7.751 \mathrm{~km}$ ) and slip checks (the part where checks were performed is zoomed in).

Figure 5.7: Data points available for this study. (a) Almost the entire traverse for Opportunity provides slip checks but the middle segment does not include processed DTM coverage. (b) Most of Spirit traverse is not available (no VO performed), but the entire traverse has processed DTM coverage. (c) Legend indicating the traverse with no slip checks, the slip checks that have DTM coverage and the slip checks that do not.

cells to derive the gradient in the rover direction (see section 5.2.2). It has indeed been shown that averaging the values of the DTM over several cells can lead to better estimate of the slope [Warren et al., 2004]. The output of this procedure is a matrix whose lines correspond to one location of slip check and its associated values of thermal inertia and slope.

\subsection{Classifier training}

Once the matrix of slope, thermal inertia and slip values at measurement locations is obtained, the data are prepared for training a classifier. It involves converting the slip data to two categories, that is, "low" and "high", which correspond to $0-30 \%$ and above $30 \%$, respectively. Bouguelia et al. (2017) chooses to divide slip into three categories, however, for the scope of this work it was decided to focus on values less or above $30 \%$. Slope and thermal inertia are also categorized into different classes. Slope categories are taken from Ono et al. (2018), where the ranges considered are $0-5^{\circ}, 5-10^{\circ}, 10-15^{\circ}, 15-20^{\circ}, 20-15^{\circ}$ and above $25^{\circ}$. However, slope 
above $15^{\circ}$ are already considered complex terrain [Biesiadecki et al., 2007], therefore only three categories of slope are considered: $0-10^{\circ}, 10-15^{\circ}$ and above $15^{\circ}$. Each category is then split to account for the direction of the slope, that is, up or down. Thermal inertia is also divided into categories, with high thermal inertia above 200 TIU and low thermal inertia below 200 TIU.

Multiple classification methods are considered, including: trees, naive Bayes or nearest neighbors. The Spirit data are used for training the classifier (referred to as the A set) and the data from Opportunity are used for validating the trained classifier (referred to as the B set). However, it should be noted that among the two categories, both for the training data and the testing data, the first category is over-represented. For all data sets combined, there is a total of 5323 slip check points with the required orbital coverage, with 4491 belonging to class "low" (84.4\%) and only 832 belonging to class "high" (15.6\%). Among the A set (from Spirit's traverse), 1600 are category "low" (77.2\%) and 473 are "high" (22.8\%). Among the B set (from Opportunity's traverse), 2891 are "low" (89\%) and 354 are "high" (11\%). This led to an imbalanced class problem for training the classifier that needed to be taken into account. There are ways to handle the class imbalance so that the model does not get biased towards the most represented category, including tuning the misclassification cost matrix or using boosting and/or sampling methods [Weiss, 2004] such as AdaBoost (Adaptive Boosting) [Liu et al., 2008] or RUS (Random Under Sampling). Boosting methods consist of creating one strong classifier, referred to as an ensemble classifier, by combining several weak ones [Opitz and Maclin, 1999] to improve performance [Rokach, 2010]. Sampling methods consists of sampling the overrepresented class to match the number of samples in the underrepresented one. Authors have also proposed to combine both sampling and boosting methods to improve even further the performance of a classifier when handling imbalanced data, called RUSboost [Seiffert et al., 2008]. When compared to different algorithms, RUSboost was proved to perform better than sampling or boosting methods alone [Seiffert et al., 2009]. Certain classifiers are better suited for such algorithms, such as decision trees, because of the high speed at which they perform [Gashler et al., 2008].

\subsection{Terrain-aware path planning}

After preparing a map containing traversability information, the next step is to plan one sol at a time by addressing path planning on segments of the traverse. Once again, the Columbia Hills are used as an example due to extensive data available at the site.

\subsubsection{Problem statement}

\subsubsection{Objectives}

The goal of this work is to develop a local path planning and real-time map update algorithm to support the autonomous traverse of a sample return rover on partially unknown terrains for the driving objective of a sol on Mars. This planning process would be repeated each sol until the traverse from the landing site to the sample locations is completed. This algorithm should take into consideration the lack of terrain information for Mars, and produce a fast and computationally efficient online replanning method to address any path issues that could arise based on terrain 
information gained along the path. The cost of a path is defined as the time of traversal from the start point to the goal for a given sol, and an optimal path is one that minimizes the traverse time.

\subsubsection{Information}

The information available to this research in the context of MSR are slip checks performed by Spirit at Gusev crater and the maps of traversability presented in section 5.2, containing information on expected velocity and slip. Definitions of the available data used to build the map are reminded below:

- Terrain types: direct output of SPOC [Rothrock et al., 2016]. At the Columbia Hills, the following are found: SR - Smooth Regolith and SO - Smooth Outcrop, RR - Rough Regolith and RO - Rough Outcrop, SRF - Sparse Ripples Firm and SRS - Sparse Ripples Sandy, DLR - Dense Linear Ripples and PR - Polygonal Ripples.

- Traversability class: each class of traversability is comprised of two terrain types (see Table 5.4). The classes are: benign (1), rough (2), sandy (3), no-Autonav (4) and untraversable (5). As a reminder, at Gusev Crater, class 4 has not been found [Rothrock et al., 2016].

- Cumulative Fractional Area (CFA): fraction of area covered by rocks [Golombek and Rapp, 1997].

- Velocity categories: lettered A to F, they represent the expected rover velocity given the slope, CFA and traversability class as they are presented in Ono et al. (2018), as shown in Fig.5.5.

\subsubsection{Constraints}

Several constraints mentioned in the overall statement apply to the path planning specifically, including a time budget (limited time each sol to perform a drive), limited computational resources for real time operations and a set daily goal from which the rover cannot deviate.

\subsubsection{Assumptions}

The main assumptions for the planning process are: the path from landing to samples is already computed and this research focuses on segments of it; obstacle avoidance is performed for small obstacles not detectable from orbit and directly in the path; the rover has reasonably accurate knowledge of its position at all times; only the traversability class data set contains uncertainty inherited from SPOC; and the rover can continuously drive for up to eight hours per sol. Even though a solar powered rover such as MERs was designed to drive at most four hours by design and in practice even less [Biesiadecki et al., 2007], better performance is expected in the future [Bajracharya et al., 2008]. Moroever, it is assumed that the computational resources are at least equivalent to those of Mars 2020 with a Virtex-5QVs Field Programmable Gate Array (FPGA) [Johnson et al., 2017] and RAD750 Single Board Computer (SBC) [Rabideau and Benowitz, 2017]. Scheduler algorithms of complexity up to $O\left(T N_{a}^{3}\right.$ ) (T number of timelines and $N_{a}$ number of activities) have been developed for such computers [Rabideau and Benowitz, 2017] and it is assumed that any algorithms of equal or lower complexity can therefore be handled by similar computational resources. Moreover, the MSR rover will not need to save energy to analyze scientific targets that would take away time otherwise allotted to driving. Finally, the assumption is made that the rover can monitor its wheel slip and actual speed, and can gather in-situ terrain information (see section 5.4). 


\subsubsection{Challenges}

A few challenges can be identified, such as the replanning approach in real time with limited computationl resources on board and limited time to finish the traverse. Another challenge is to convert terrain monitoring into terrain information that can be used in planning. Finally, determining if the path ahead is safe to traverse is the most challenging task the rover will have to face.

\subsubsection{Contributions}

The main contribution of this work is a novel terrain-aware planning framework that supports the MSR mission concept by enabling a rover to drive safely over significant distances (beyond line-of-sight). It includes:

- A planning algorithm for Mars rovers that can incorporate orbital terrain information, such as class, presence of rocks, and slope, to allow the rover to autonomously assess its environment prior to driving.

- An algorithm that converts rover parameters (slip, speed) into terrain information to lower the uncertainty about the terrain.

- A planning process that gives the vehicle options to reroute if a more efficient path is found without requiring heavy real-time computation, resulting in faster decision making and replanning.

\subsubsection{Approach}

The path planning process is split into an offline path planning phase and an online map update and replanning loop. This approach was chosen to alleviate, as much as possible, the use of computational resources onboard. The offline planning creates paths for the rover to use during its traverse, whereas the online part is dedicated to measurements of the terrain and map updates, given new terrain information, with possible replanning if needed (i.e., terrain has changed significantly). The overall process is presented in Fig.5.8 and detailed in the next subsections.

\subsubsection{Path planning approach}

To reduce the computational cost online, several paths are computed offline prior to begin the traverse of a sol, to give the rover options to reroute in real time if necessary. This approach enables the rover to quickly replan its traverse without involving heavy computations, should the terrain be different than expected. Rerouting could be triggered by any significant change detected by the rover as it drives and it is assumed that various methods such as slip monitoring or instrument deployment can detect terrain variations. The start and goal locations are chosen so that the rover would not take more than eight hours to drive from one to the other (Fig.6.6). To plan the paths, a graph is first built using a Probabilistic Road Map (PRM [Kavraki et al., 1996]) and an A* algorithm [Stentz, 1993] then computes paths from start to goal. The cost of the traverse is the sum of the cost of the edges along the path given by Eq.5.4.

$$
e_{c}=\frac{l_{e}}{V_{e}}
$$

Where $e_{c}$ is the edge cost (in hours), $l_{e}$ is the length of an edge in meters and $V_{e}$ is the maximum velocity encountered by the rover along the edge (in $m / h r$ ). This ensures that the heuristic chosen 


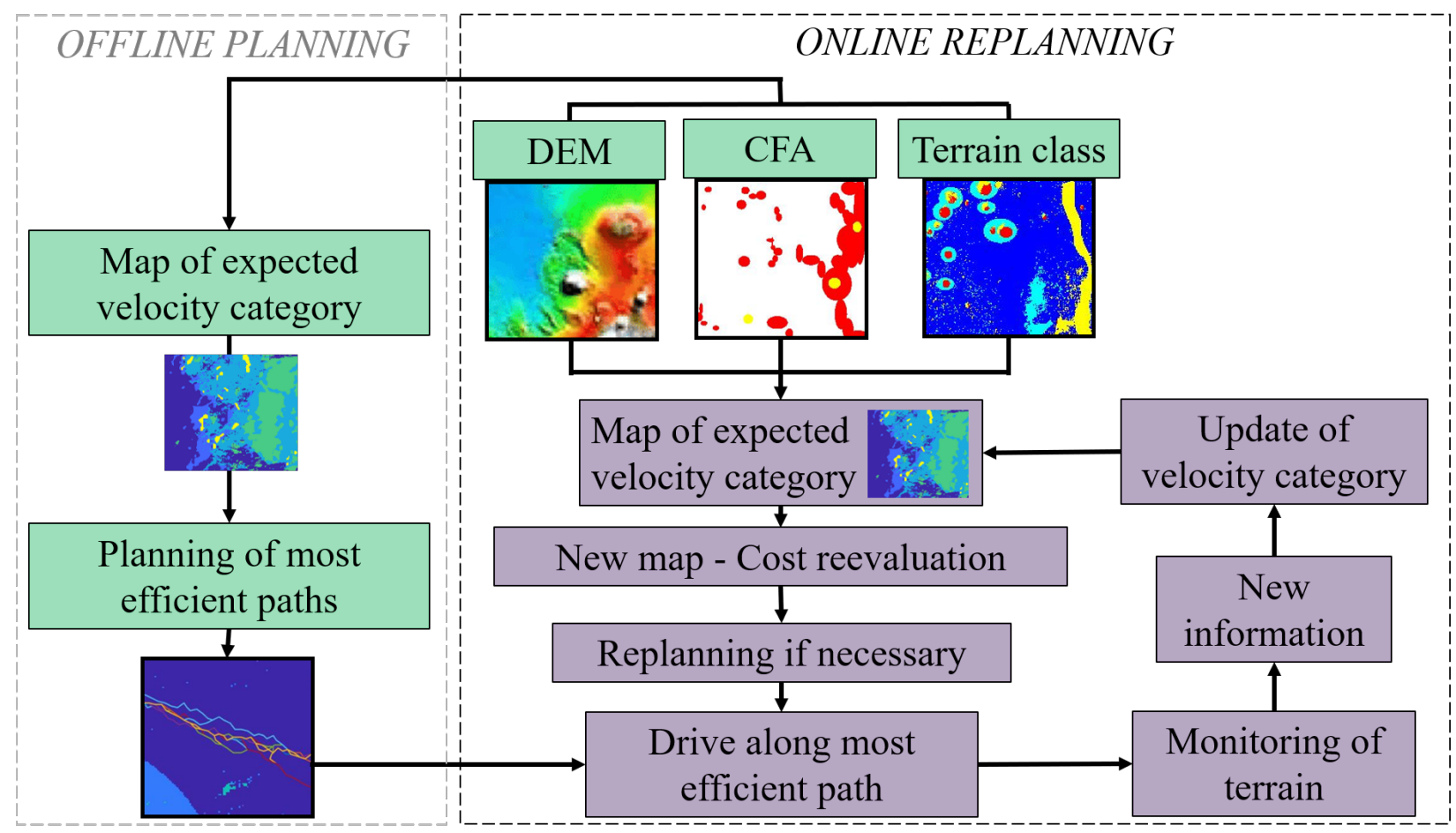

Figure 5.8: Diagram showing the one-time offline planning and the online loop comprised of update and replanning Credit: Hedrick et al., 2020.

is admissible, which means that it is never overestimated. Each cell corresponds to a pixel on the orbital images, with a resolution of $1 \mathrm{~m} / \mathrm{px}$. The velocity over a pixel is obtained from Rothrock et al. (2016) by overlapping the slope, traversability class, and the rock abundance for each cell (see subsection 5.2.3.1). The slope is derived from the DTM using Horn's method in the direction of driving (simplified to "up" or "down") as shown in Ono et al. (2018). The traversability class and CFA values are obtained from the maps described in subsection 5.2.3.2. To compute several paths to the goal, Yen's k-shortest paths algorithm [Yen, 1970] is implemented on the node following the spur node of the latest computed path. The algorithm was modified to incorporate the following: maximum cost of each path (i.e., time budget); and minimum distance from each node on previous paths to avoid computing paths too close to each other. The number of paths with this no-go corridor around it can be changed, and the width of the corridor cannot be longer than twice the maximum length of an edge. With this method, the maximum number of options generated is the number of edges of the second-to-last computed path, and it allows for both diversity and overlapping of the paths.

\subsubsection{Terrain monitoring: noisy measurements}

The terrain monitoring on the nodes along the path can be referred to as "noisy measurement", which is a measurement of conditions corresponding to a specific Probability Mass Function (PMF) of velocity categories $C$ given wheel slip and drive speed. It is assumed that the rover has access to its wheel slip $S$ and actual speed $V$ while driving, both of which are then converted into 
terrain information with the method described below.

The PMF of velocity category given slip and speed, $p\left(C_{i} \mid S_{i} \cap V_{i}\right)$, can be expressed using Bayes rule, where $S_{i}$ is the measured slip at cell $i$ and $V_{i}$ is the velocity observed at cell $i$. Bayes rule gives:

$$
p\left(C_{i} \mid S_{i} \cap V_{i}\right)=\frac{p\left(S_{i} \cap V_{i} \mid C_{i}\right) * p\left(C_{i}\right)}{p\left(S_{i} \cap V_{i}\right)}
$$

where $p\left(S_{i} \cap V_{i}\right)$ is derived from $p\left(S_{i}\right)$ and $p\left(V_{i}\right)$ and $p\left(S_{i} \cap V_{i} \mid C_{i}\right)$ is derived from $p\left(S_{i} \mid C i\right)$ and $p\left(V_{i} \mid C_{i}\right)$.

$p\left(S_{i}\right)$ and $p\left(S_{i} \mid C_{i}\right)$ are obtained from Spirit's slip checks at Gusev Crater used in section 5.2. Given the availability of a velocity category map at the Columbia Hills, slip distributions over each velocity category are studied. The distributions presented in this section were analyzed using the manually interpreted map described in subsection 5.2.3. The PDFs for slip are derived by fitting a curve to the data for each categories, except for E, not represented at Gusev. Slip seems to follow a log-normal distribution for which parameters are presented in Table 5.2. The exception to the rule is category $\mathrm{F}$ which shows two maxima (see Fig.5.10a), including one in the very high slip region (to be expected).

Table 5.2: Slip probability distributions parameter, per velocity category, derived from Spirit's slip checks

\begin{tabular}{|c|c|c|c|}
\hline $\begin{array}{c}\text { Velocity } \\
\text { Category }\end{array}$ & Mean (\%) & $\begin{array}{c}\text { Standard } \\
\text { Deviation }\end{array}$ & PDF \\
\hline \hline A & 2.7812 & 0.92170 & Log-normal \\
\hline B & 1.5054 & 0.77050 & Log-normal \\
\hline C & 2.0309 & 0.67210 & Log-normal \\
\hline D & 1.4897 & 0.55190 & Log-normal \\
\hline
\end{tabular}

The variation of speed within each category has not been reported in Ono et al. (2018). To obtain the PDFs of each category and understand how the velocity changes from A to $\mathrm{F}$ and varies within each category, the following process is applied:

- If the category is described by a range, a Gaussian distribution is assumed. The mean is taken and the range is considered to cover a total of six standard deviations (since $97 \%$ of the data lie within three standard deviations of the mean).

- If the category is described by a single number, however, assumptions on the distribution as well as maximum and minimum velocities are made. The number given in Ono et al. (2018) is taken for the mean, and maximum values are obtained from different drive modes' maximum attainable velocity as described in Biesiadecki et al. (2007). It is assumed that each drive mode corresponds to one specific category, such as blind drive being possible only on category A terrain.

The assumption for the best fit distribution is assumed to be Gaussian for category A to C and $\log$ normal for category F. The reason for choosing log-normal distribution for lower speed category is to ensure that most data points will fall under a very low range of velocity in accordance with 
difficulties created by the terrain class, the slope and/or the rock abundance. Given the conditions required for the rover to drive within category $\mathrm{F}$, it is unlikely that the rover will achieve greater speeds. A log-normal distribution also ensures that the data points remain in the positive range. It is to be noted once again that in this work, there is no category E at Gusev Crater. The following table summarizes the numbers used to build the velocity PDFs for categories with only one given value: The illustration of a distribution of velocity and slip for each traversability class is shown in

Table 5.3: Velocity categories and their characteristics for those with only one given number [Ono et al., 2018]

\begin{tabular}{|c|c|c|c|c|}
\hline Category & Mean & $\begin{array}{c}\text { Maximum [Biesiadecki } \\
\text { et al., 2007] }\end{array}$ & $\begin{array}{c}\text { Standard } \\
\text { Deviation }\end{array}$ & PDF \\
\hline \hline A & $64.8 \mathrm{~m} / \mathrm{hr}$ & $124 \mathrm{~m} / \mathrm{hr}$ & $\frac{124-64.8}{3}$ & Normal \\
\hline B & $54.5 \mathrm{~m} / \mathrm{hr}$ & $96.0 \mathrm{~m} / \mathrm{hr}$ & $\frac{96-54.5}{3}$ & Normal \\
\hline F & $0.00 \mathrm{~m} / \mathrm{hr}$ & $10.0 \mathrm{~m} / \mathrm{hr}$ & $\frac{10}{3}$ & Log-normal \\
\hline
\end{tabular}

Fig.5.9 with category A given as an example.
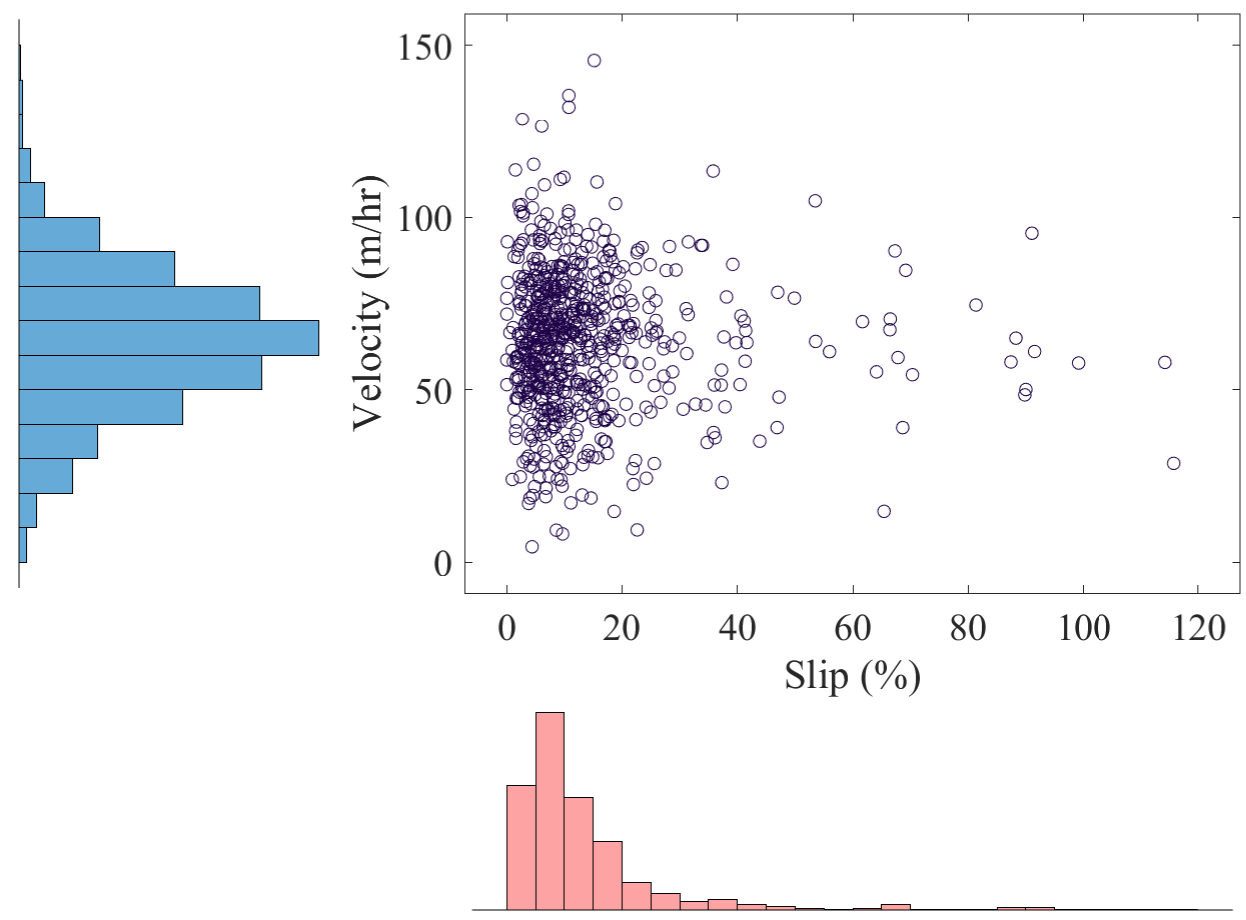

Figure 5.9: Slip vs. velocity with marginal distribution over category A (encompasses terrain types 1 and 2).

The distributions are plotted and shown in Fig.5.10. 


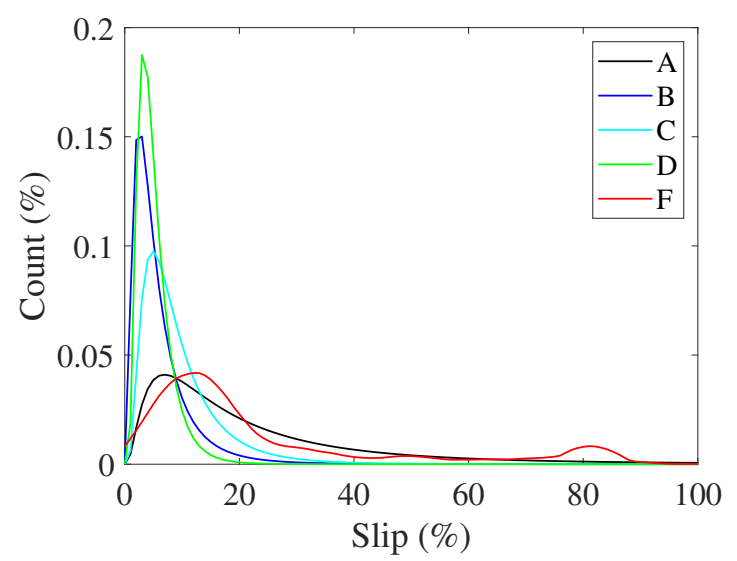

(a) PDF of slip over each category (A to F).

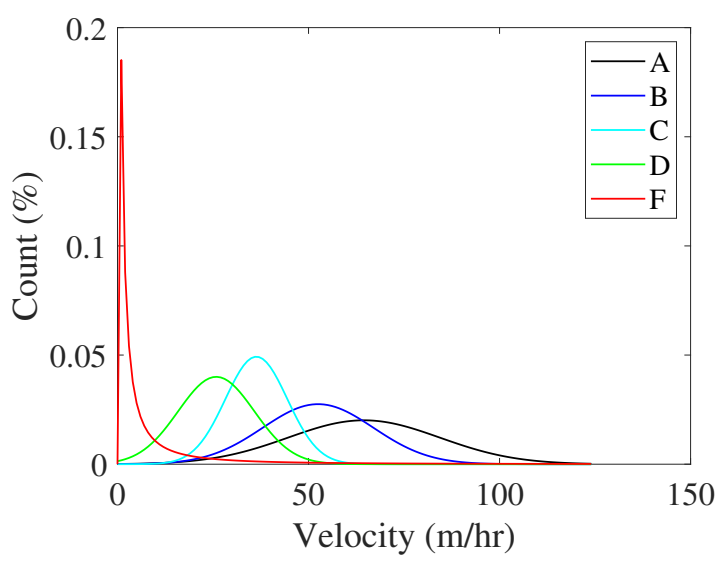

(b) PDF of velocity over each category (A to F).

Figure 5.10: PDFs of (a) slip and (b) speed for each velocity category.

Once the PDFs for velocity and slip are established over the entire map, as well as the PDFs for each category, the joint probabilities $p\left(S_{i} \cap V_{i}\right)$ and $p\left(S_{i} \cap V_{i} \mid C_{w_{i}}\right)$ can be computed using a partition in 2D-bin counts with specific bin edges in both dimensions. A matrix is obtained, with the lines corresponding to the slip dimension and the columns corresponding to the velocity dimension. The cells are populated given the probability of slip and velocity to fall within the specific bin given by the line number and column number, respectively.

Assuming that the rover can monitor its own wheel slip and actual speed, at each measurement $i$ it will convert this information into a PMF of category by simply calculating the probability that the measurement $(S, V)_{i}$ falls into one of the categories, given the combined values of slip and speed as stated in Eq.5.5. For simulation purposes, these "measurements" are computed from an altered map referred to as the "truth" map $(1 \mathrm{~m} / \mathrm{px})$. The alterations were made so that the map remains as close as possible to real situations: for example, class 1 has $10.5 \%$ chance to be mistaken for class 2, but class 6 can hardly be misclassified, with a prediction accuracy of $99.8 \%$ [Rothrock et al., 2016] (Table 5.4).

\subsubsection{Map and map update}

The map known to the rover prior to planning is a belief map, specifying the probability distribution over the expected velocity categories of each of the grid cells. This belief map is built from the map of velocity obtained in subsection 5.2.3 by initializing with the PMF of the velocity of each pixel. The derivation of the PMFs is presented in the next subsections.

\subsubsection{Belief map}

\subsection{Class confusion matrix}

The uncertainty contained in the traversability class map are derived from the SPOC uncertainty presented in Rothrock et al. (2016). The class confusion matrix (Table 5.4), i.e., the matrix showing the performance of the terrain classifier, has been adapted to traversability classes instead of terrain types as publised in Rothrock et al. (2016) by implementing the following steps: 
- The proportion of each class present at the Columbia Hills was computed by simply adding the proportions of each terrain belonging to the class:

$$
p_{c_{i}}=\sum_{j} p_{t_{j} \subset c_{i}}
$$

where $p_{c_{i}}$ is the proportion of class $i$ and $p_{t_{j} \subset c_{i}}$ is the proportion of terrain type $j$ included in class $i$.

- Each terrain proportion within a class was calculated using the following formula:

$$
p_{t_{i} \subset c_{j}}=\frac{p_{T_{i}}}{p_{c_{j}}}
$$

where $p_{t_{i}} \subset c_{j}$ is the proportion of terrain type $i$ in class $j, p_{T_{i}}$ is the proportion of terrain type $i$ at the Columbia Hills as observed by Rothrock et al. (2016) and $p_{c_{j}}$ is the proportion of class $j$ at the Columbia Hills as calculated previously.

- Each value within the confusion matrix was calculated based on the proportion of each terrain within each class and the probability given by the confusion matrix for that terrain in Rothrock et al. (2016):

$$
P\left(c_{j}\right)=\sum_{i} p_{t_{i} \subset c_{j}} P\left(t_{i}\right)
$$

where $P\left(c_{j}\right)$ is the performance of the model for class $j$ (computed as explained above), $p_{t_{i}} \subset c_{j}$ is the proportion of terrain type $i$ in class $j$ and $P\left(t_{i}\right)$ is the performance of the model for terrain type $i$ (from Rothrock et al. (2016)).

Table 5.4: Confusion matrix for traversability classes (1 to 5 [Ono et al., 2018]) derived from Rothrock et al. (2016). Terrains included in the classes are: SR - Smooth Regolith, SO - Smooth Outcrop (class 1), RR - Rough Regolith, RO - Rough Outcrop (class 2), SRF - Sparse Ripples Firm, SRS - Sparse Ripples Sandy (class 3), DLR - Dense Linear Ripples, and PR - Polygonal Ripples (class 5). As specified before, no class 4 was detected at the Columbia Hills [Rothrock et al., 2016].

\begin{tabular}{|l|c|c|c|c|c|}
\cline { 3 - 6 } \multicolumn{2}{c|}{} & \multicolumn{4}{c|}{ Prediction } \\
\cline { 3 - 6 } \multicolumn{2}{c|}{} & SR+SO class 1 & RR+RO class 2 & SRF+SRS class 3 & DLR+PR class 5 \\
\hline \multirow{3}{*}{$\begin{array}{l}\text { Ground } \\
\text { Truth }\end{array}$} & SR+SO & $84.0 \%$ & $7.50 \%$ & $0.200 \%$ & $0 \%$ \\
\cline { 2 - 6 } & RR+RO & $10.5 \%$ & $81.3 \%$ & $1.20 \%$ & $0.200 \%$ \\
\cline { 2 - 6 } & SRF+SRS & $5.20 \%$ & $10.6 \%$ & $98.4 \%$ & $0 \%$ \\
\cline { 2 - 6 } & DLR+PR & $0.300 \%$ & $0.600 \%$ & $0.200 \%$ & $99.8 \%$ \\
\hline
\end{tabular}

Given this confusion matrix for each traversability class, the PMFs for the velocity categories can be derived under the assumption that only the traversability class set contains uncertainty.

\subsection{Probability Mass Functions (PMFs) of velocity categories}

For each category (see Fig.5.5), it is first determined what classes of terrain are included, what the proportions of each class within the category are, and finally, the probability that the prediction matches the ground truth is calculated. Each column of the matrices corresponds to the belief PMF of velocity categories for each combination of CFA and slope and each row is the "true" 
PMF of categories (i.e., the "measurement"). To illustrate the derivation, an example is taken for CFA less than $7 \%$ and slope between $20^{\circ}$ and $25^{\circ}$. This combination of CFA and slope gives only two possible categories of velocity: E $(10.9 \mathrm{~m} / \mathrm{hr}$ ) and $\mathrm{F}$ (untraversable). E includes class 1 and 2. Moreover, within these ranges of CFA and slope, category F can only be class 3 or 5. To calculate the probability that category $\mathrm{E}$ is mistaken for $\mathrm{F}$, the following steps are followed:

- The relative proportions of class 1 and class 2 within $\mathrm{E}$ are obtained, called $p_{c_{1} \subset E}$ and $p_{c_{2} \subset E}$, respectively:

$$
p_{c_{i} \subset C_{j}}=\frac{\sum_{k} p_{t_{k} \subset c_{i}}}{\sum_{i} p_{c_{i}}}
$$

where $p_{c_{i} \subset C_{j}}$ is the proportion of class $c_{i}$ within category $C_{j}, p_{t_{k} \subset c_{i}}$ is the proportion of terrain type $k$ within class $c_{i}$ and $p_{c_{i}}$ is the proportion of class $i$ at the site of interest, calculated from Eq.5.6.

- The relative proportions of class 3 and class 5 within $\mathrm{F}$ is calculated, called $p_{c_{3} \subset F}$ and $p_{c_{5} \subset F}$, respectively.

- The numbers in Table 5.4 corresponding to the performance of the model for each predicted class ( 1 and 2) compared against each true class ( 3 and 5) is taken, called $P\left(c_{1} \mid c_{3}\right)$ (probability of the model to give class 1 knowing that it is in fact 3), $P\left(c_{1} \mid c_{5}\right), P\left(c_{2} \mid c_{3}\right)$ and $P\left(c_{2} \mid c_{5}\right)$.

The final equation is the following:

$$
\begin{array}{r}
P(E \mid F)=p_{c_{1} \subset E}\left(p_{c_{3} \subset F} * P\left(c_{1} \mid c_{3}\right)+p_{c_{5} \subset F} * P\left(c_{1} \mid c_{5}\right)\right)+ \\
p_{c_{2} \subset E}\left(p_{c_{3} \subset F} * P\left(c_{2} \mid c_{3}\right)+p_{c_{5} \subset F} * P\left(c_{2} \mid c_{5}\right)\right)
\end{array}
$$

Which gives, in this case:

$0.54(0.436 * 5.2 \%+0.564 * 0.3 \%)+0.46(0.436 * 10.6 \%+0.564 * 0.6 \%)=8.31 \%$.

Table 5.5: $\mathrm{CFA} \leq 7 \%$ and $20^{\circ}<$ slope $\leq 25^{\circ}$.

\begin{tabular}{|l|c|c|c|}
\cline { 3 - 4 } \multicolumn{2}{c|}{} & \multicolumn{2}{c|}{ Prediction } \\
\cline { 3 - 4 } \multicolumn{2}{c|}{} & $\mathrm{E}$ & $\mathrm{F}$ \\
\hline \multirow{2}{*}{ Truth } & $\mathrm{E}$ & $82.8 \%$ & $.340 \%$ \\
\cline { 2 - 4 } & $\mathrm{F}$ & $8.31 \%$ & $99.2 \%$ \\
\hline
\end{tabular}

This number is entered in Table 5.5. The other cells are calculated using a similar process (rows and columns of zeros are not shown for clarity purposes). All of the matrices corresponding to different values of slope and CFA are presented in Appendix B

\subsection{Belief map of velocity category}

Given the matrices of PMFs, the map of velocity category from subsection 5.2.3 is initialized by taking the column of each matrix (believed PMFs) corresponding to the category of each pixel of the map given CFA and slope. For example, if a pixel has a CFA less than 7\%, slope under $10^{\circ}$ and the initial map obtained in subsection 5.2.3 shows category A for that pixel, then its value on the belief map will be the corresponding PMF of [0.840, 0, 0.105, 0.0520, 0, 0.000300]. 


\subsubsection{Local Map Update}

The map update allows the rover to keep a local up-to-date velocity map at $1 \mathrm{~m} / \mathrm{px}$ using constant monitoring of the terrain while driving (see section 5.3.2.2). The belief map known to the rover contains uncertainty due to the terrain classifier errors [Rothrock et al., 2016]. This work has been done in collaboration with Nicholas Ohi at WVUIRL.

\subsection{Edge potential matrices}

The edge potential matrices, representing the conditional relations between velocity categories in adjacent nodes on the underlying PRM graph, are built after the PMF matrices derived in subsection 5.3.3.1.2 and presented in Appendix B. When used as edge potentials in the map update framework, the columns of the matrices of PMFs are normalized, so each sums to $100 \%$.

\subsection{Belief propagation framework}

Each cell $s$ in the map has an associated belief state of the velocity category of that cell $\phi(s)$. The initial belief state of a cell is derived from the PMF of velocity categories, by tuning the probabilities towards the most likely categories as seen from ground images assumed to be available to MSR from Perseverance. Here, for simulation purposes, the truth map is used to set the initial conditions.

The dimensionality of the problem is reduced by estimating the belief at the nodes of the PRM graph used for path planning in the local area around the location where the measurement was taken. The graph used for belief propagation is found as a minimum spanning tree of the subgraph of the PRM graph (with a time complexity of $O(|E| \log |\nu|$ ), where $|E|$ is the number of edges in the graph and $|\nu|$ the number of nodes), consisting of the subset of nodes less than or equal to a graph distance of $N$ away from the measured node. This minimum spanning tree of the local subgraph around the measurement is referred to as the belief propagation tree, the nodes of this graph are the set $\{x\}$, and the belief state of a particular node $x_{i}$ is $\phi\left(x_{i}\right)$, representing a Markov Random Field (MRF). The belief of the rest of the cells in the local area of the map is then updated by linearly interpolating between nodes in the belief propagation tree.

The edge potential functions $\psi\left(x_{i}, x_{j}\right)$ represent conditional relations between neighboring nodes in the graph and describe how the state of $x_{j}$ should be updated, based on the state of $x_{i}$. A measurement taken at a particular node $z_{i}$ directly results in a new belief state for that node $\phi\left(x_{i} \mid z_{i}\right)$. The objective of the map update is to propagate this new information to the rest of the graph and infer the marginal PMFs of each node. In other words, the algorithm updates the belief states of all other nodes, given the measurement, $\phi\left(x_{j} \mid z_{i}\right), \forall j \neq i$. The minimum spanning tree of the local subgraph is found, so that exact inference can be performed using the sum-product message passing algorithm [Kschischang et al., 2001] described in section 4.2.5.1. The belief propagation is implemented with the UGM Matlab ${ }^{\circledR}$ Toolbox [Schmidt, 2012]: the belief propagation tree (i.e., MRF) is first factorized, and given the measured node (message to be passed), the marginal distributions of each leaf are then computed using equations 4.4, 4.5 and 4.6, leading to a complexity of $O\left(|\nu|^{2}\right)$ ( $\nu$ vertices of the tree, in this work $|\nu|<100$ ). If a tree leaf overlaps with a previously measured node, the measurements overwrite any information passed on to the leaf, under the assumption that the measurements, even noisy, are more accurate than the updated information resulting from the message passing. Those nodes (currently and previously measured) are referred to as clamped nodes in the belief propagation algorithm. 


\subsubsection{Replanning}

Replanning is used to optimize the remaining time to the goal. After each map update, if the most likely velocity category along the path the rover is driving on is different than the believed velocity, the costs of the pre-planned paths are recalculated using Eq.5.4 with the new velocities. If there is an available path among the proposed options with a lower cost than the current path the rover is following, and if there is a connection (i.e., a segment of a pre-computed path from the rover's location to the potential new path) this option is selected to continue the traverse. The complexity of the replanning algorithm is simply $O(n \log |\nu|)$ in time and $O(\log \mid \nu) \mid$ in space, where $\nu$ are the vertices on the paths.

\subsection{Parameters and instrumentation to assess terrains}

As previously mentioned, there is a need for increased autonomous driving distance due to the potential size of the landing ellipse and the plan for a duration of only 100 to 200 sols for the MSR mission [Klein et al., 2014]. While this research proposes methods to increase autonomy, such as a map of expected mobility performance and a path planning that fully takes into account the environment, there might still be need for a more accurate terrain assessment when all the other methods have failed to correctly capture the situation. If the terrain monitoring referred to as noisy measurements is not enough to confirm that the terrain ahead is safe, or if there is a confusion between terrains, including one that could present mobility difficulties, the rover needs to have access to a different method to assess the terrain and support planning.

\subsubsection{Problem statement}

\subsubsection{Objectives}

The objective is to equip the rover with the capability of gathering in-situ terrain information in a timely manner (i.e., a few minutes) to support planning, at an accuracy equal to or greater than a human operator. It would allow the vehicle to make decisions regarding its traverse when slip and speed monitoring is not sufficient to confirm a safe path, and/or when a dangerous terrain is detected in the vicinity of the rover. This new knowledge would be used to update the map with lower uncertainty using the belief propagation framework described in section 5.3.3.

\subsubsection{Information}

The information available are the rover wheel slip and actual drive speed (i.e., noisy measurements). For simulation purposes, the noisy measurements are implemented by simply taking the most likely values for slip and speed for each category the rover will traverse according to the truth map. A map of expected velocity and expected slip, locally updated after noisy measurements, is also available (see section 5.2).

\subsubsection{Constraints}

The main constraint is to choose an instrument that meets the following requirements: it can be adapted to operate remotely, it can be easily deployed and the output can be processed fast, to support planning in a timely manner. MSR's primary mission is not to explore, but to retrieve 
samples left by Perseverance, thus it needs to be fast and efficient. The method of choice to gather in-situ information needs to reflect the mission's goal.

\subsubsection{Assumptions}

An important assumption in assessing the results of experimentation is that everything applicable to experiments on Earth is also applicable to experiments on Mars. An instrument on Earth and its manipulation on a given terrain will result in similar conclusions when operated on Mars on a similar soil. For example, a cone penetration test in sand is interpreted in the same manner on both planets. Results are thus assumed to be valid when carried out on Mars. In addition, it is assumed that a terrain is "dangerous" when falling within categories D to F. While it might not present an actual danger to the rover (e.g., moderate ripples with firm substrate), the risk of encountering mobility difficulties is higher on such terrains than on traversability classes one and two at slopes lower than $20^{\circ}$ (i.e., velocity categories A to C).

\subsubsection{Challenges}

The main challenge is to have experiments widely applied on Earth adapted to a rover. Any tool chosen needs to be mounted on the rover, and any experiments must be performed remotely, regardless of terrain conditions (i.e., flat, tilted, etc...).

\subsubsection{Contributions}

The main contributions of this work are the following:

- To provide an easy option to gather intrinsic soil parameters that can be used to identify a terrain when needed.

- To give a way of identifying a terrain with little to no human intervention.

- To obtain results in a timely manner (i.e., a few minutes) to allow a fast traversing rover to quickly make a decision regarding its path given the terrain characteristics.

\subsubsection{Instrument to assess terrain properties}

\subsubsection{Choice of instrumentation}

The two instruments considered to support in-situ terrain assessment are the cone penetrometer (see Fig.2.5) that can give access to cohesion, angle of internal friction and ultimate bearing capacity, and the shear vane (see Fig.2.6), which can indicate the shear strength and bearing capacity of a soil.

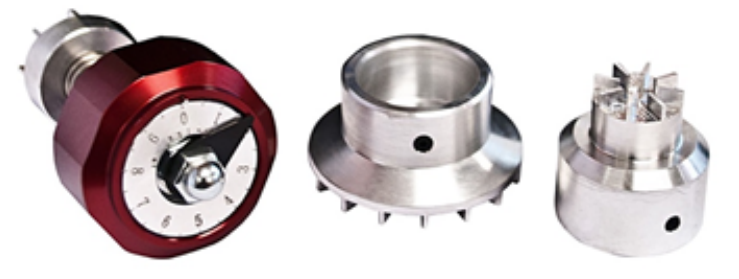

Figure 5.11: Pocket shear vane with standard blades (left) and two other sizes of blades. Credit: Gilson inc.
While the geovane and field shear vane are lengthy instruments, similar to the cone penetrometer, the pocket shear vane is a light weight, compact tool, ideal for remote operations on planetary rovers (Fig.5.11). It also gives outputs that can be easily processed, as they do not require additional parameters to be estimated (e.g., bearing capacity factors needed for Eq.2.4 for calculating $c$ and $\phi$ with the cone penetrometer). The advantage of the penetrometer, however, is to give cohesion and angle of internal friction, which play an essential role in 
identifying a terrain that the bearing capacity cannot fulfill.

Rahmatian and Metzger (2010) have suggested modifying the pocket shear vane to obtain several normal stresses and their corresponding shear stresses, which would lead to a MohrCoulomb linearized envelope, thus giving the cohesion and angle of internal friction of a soil (Fig.2.4). The failure point being of interest, along with its corresponding Mohr-Coulomb criterion, the linearization is an acceptable approximation for this research [Labuz and Zang, 2012]. Therefore, the pocket shear vane is selected to support path planning and terrain assessment as a base instrument for building a modified version that could give the desired $c$ and $\phi$. In general, the pocket shear vane seems to better meet the requirements of a fast deployment and an easy analysis of the results compared to other possible instruments (e.g., a spectrometer, that can take up to three hours just for deployment [Gellert et al., 2009], or even a cone penetrometer). Moreover, for a rover on Martian soil, assumptions can be made that the confining pressure $\sigma_{3}$ is negligible and the vertical stress $\sigma_{1}$ is simply the load of the vehicle [Sullivan et al., 2011], as illustrated in Fig.5.12. With these assumptions, the Mohr circle at failure (i.e., the circle whose tangent is the Mohr-Coulomb envelope) gives the maximum shear stress $\tau_{\max }$ (or wheel rotation at constant velocity [Sullivan et al., 2011]) and the maximum load (or weight) the soil can bear, which is by definition the ultimate bearing capacity $q_{u l t}$ (Fig.5.12).

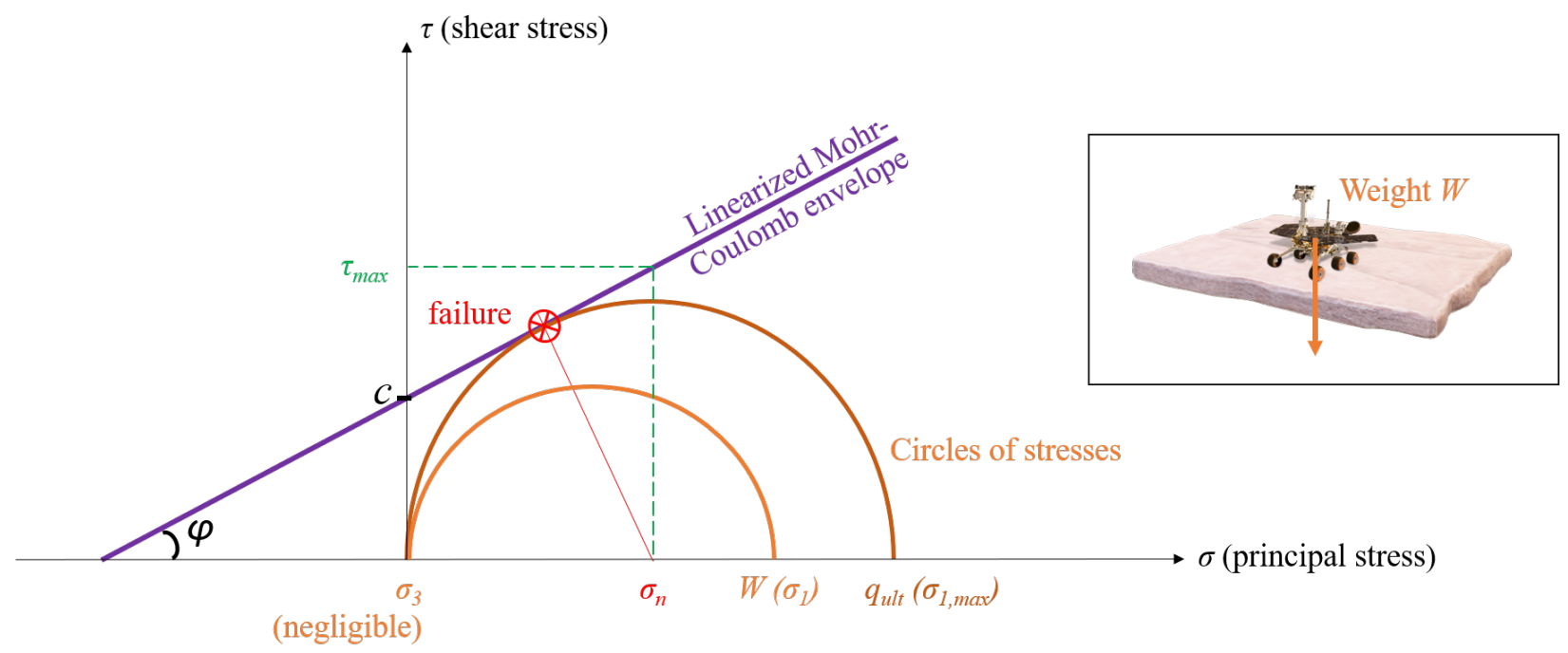

Figure 5.12: Mohr-Coulomb envelope and failure point shown for a vertical load (rover weight) on a terrain. The confining pressure is negligible [Sullivan et al., 2011]. When reaching failure, $\sigma_{1}$ represents the maximum load the terrain can sustain, which is by definition the ultimate bearing capacity.

\subsubsection{Hand held pocket shear vane}

The pocket shear vane gives only the value for shear stress (i.e., $\tau$ ), while the vertical stress is applied by a human operator. As suggested in Rahmatian and Metzger (2010), the pocket shear vane can be modified to bear a controlled amount of weight (and therefore, a controlled normal stress can be applied). The modification is shown in Fig.5.13 and consists of a 3D printed cast with slots tailored to calibration weights. The maximum total weight available is $0.55 \mathrm{~kg}$ and the minimum is $0.17 \mathrm{~kg}$. By applying different weights to obtain several measurements at the same 


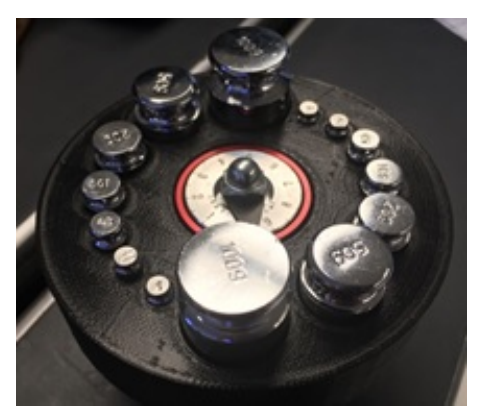

Figure 5.13: Modified pocket shear vane tester including: a 3D printed cast with weight slots; calibration weights.

location, a range of normal stresses and their associated shear stresses can be plotted. The MohrCoulomb envelope can be obtained from these measurements and the resulting slope and y-axis intersection would give $\phi$ and $c$, respectively.

\subsubsection{Mounted shear vane}

To make the pocket shear vane remotely operable, the human actions must be replicated by electronic motions and the stresses obtained digitally. The process is detailed in the subsequent paragraphs and has been performed in collaboration with Dylan Covell at WVUIRL.

As seen in Fig.5.14, the instrument is integrated into an actuated payload equipped with a potentiometer and a pair of shear load cells oriented perpendicular to each other. This prototype was designed to minimize undesired skew and payload footprint, while remaining simple to manufacture. The T-slot guide rails provide an adaptable means of connecting this sensor to the IRL's autonomous Fast Traversing Rover (FTR) for Mars sample collection while allowing the instrument to be tested independently. Moreover, the T-slot linear bearings contain no moving parts, an ideal trait for the dusty Martian environment. The linear servos are used over a stepper motor and lead screw to protect from dust and debris.

Once the payload frame is resting on the ground, a linear servo presses down on the shear vane and the resulting normal force is read via the load cell oriented perpendicular to the ground. The output voltage of the load cell is amplified before reaching the arduino, which is then output as an equivalent weight by the HX711 library. The rated repeatability of the load cells is $+/-10 \mathrm{~g}$ or $0.05 \%$ of the maximum range of $20 \mathrm{~kg}$. This weight value is converted to a normal stress using Eq.5.11:

$$
\sigma_{n}=\frac{W g}{S}
$$

Where $\sigma_{n}$ is the normal stress, $W$ is the weight of the instrument, $g$ is the gravity $\left(9.81 \mathrm{~m} / \mathrm{s}^{2}\right.$ for Earth) and $S$ is the surface covered by the blades. $S$ is specified in the instrument's instruction manual and is equal to $0.000491 \mathrm{~m}^{2}$. The measurement from the shear vane is given in $\mathrm{kg} / \mathrm{cm}^{2}$ and converted to $\mathrm{kg} / \mathrm{m}^{2}$. The shear stress is obtained from the measurement simply by multiplying by $g$.

The resulting shear stress can then be obtained via two methods: the potentiometer output, that models the position of the shear vane dial; and the output of the load cell, aligned with the torsional neutral axis, is amplified before the arduino reads the voltage. The HX711 library takes this 
voltage and outputs an equivalent weight, which is then converted to a shear stress using Eq.5.12:

$$
\tau=\frac{W * d * r}{J}
$$

Where $\tau$ is the shear stress $(P a), W$ is the measured weight $(N), d=0.02 m$ is the distance between the effective weight $W$ and the neutral axis, $r=0.00635 \mathrm{~m}$ is the distance between the center of the load cell and the stressed surface, and $J=1.8221 \times 10^{-9} \mathrm{~m}^{4}$ is the polar moment of inertia. Therefore, Eq.5.12 can simply be rewritten as:

$$
\tau=6.97 \times 10^{4} \mathrm{~W}
$$

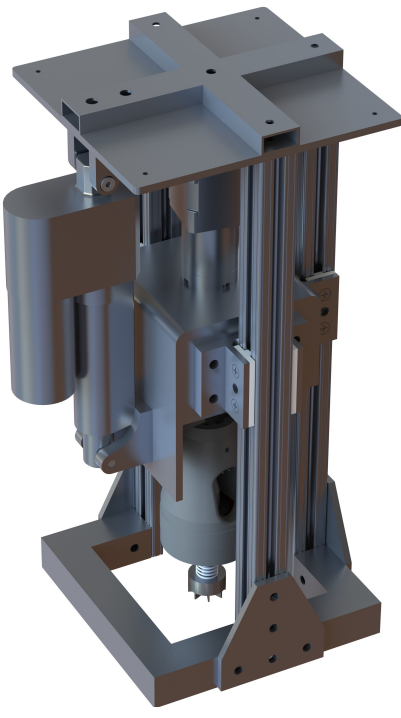

(a) Refined payload prototype

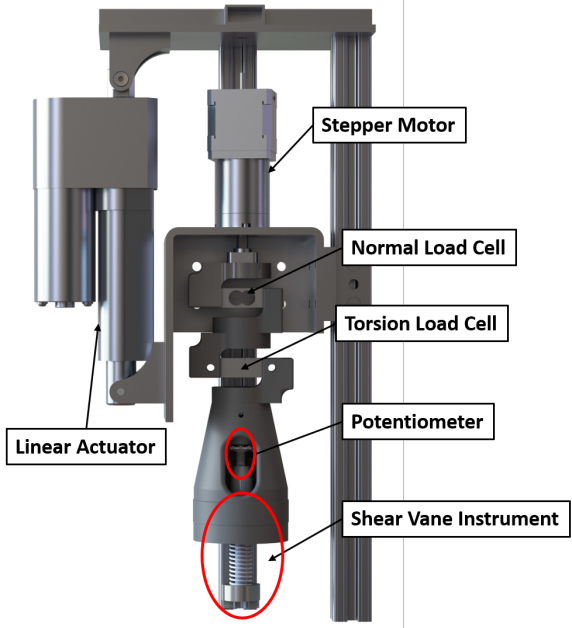

(b) Main components in refined payload.

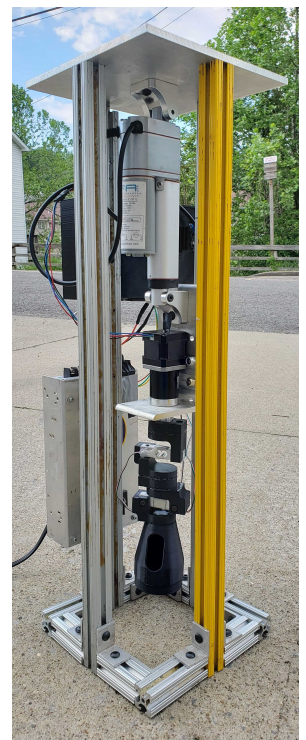

(c) Current physical prototype.

Figure 5.14: Automated shear vane test prototype. Credit: D. Covell.

This prototype is composed of the electronic hardware found in Table 5.6 and controlled via MATLAB $^{\circledR}$ through an Arduino Uno. The Arduino library for the load cell amplifier [Giacoboni, 2020] greatly enables a seamless integration of the load cells into MATLAB ${ }^{\circledR}$. The first prototype of the refined payload shown inside the center rover compartment (Fig.5.15) is currently estimated to weigh $7.3 \mathrm{~kg}$ and take up a $39.4 \mathrm{~cm}$ tall $\mathrm{x} 16.8 \mathrm{~cm}$ wide $\mathrm{x} 22.9 \mathrm{~cm}$ deep box.

\subsubsection{Instrument deployment}

The instrument is to be deployed if monitoring slip and speed is not sufficient to assess the terrain. There are two main cases in which such an exact measurement would be necessary:

- A dangerous terrain is detected ahead: anything that falls under category D to F (see Assumption subsection 5.4.1.4) with a probability greater than 75\%. This type of exact measurement trigger will be referred to as "danger". 
Table 5.6: Prototype Hardware and Estimated Maximum Energy Consumption. Credit: D. Covell.

\begin{tabular}{||c|c|c||}
\hline Component & $\begin{array}{c}\text { Maximum Power } \\
\text { Consumption }(W)\end{array}$ & Company \\
\hline Arduino Uno R3 & 2.5 & Arduino \\
\hline $\begin{array}{c}\text { CTS 282T33L502A26C2 } \\
\text { Potentiometer }\end{array}$ & 0.005 & Digi-Key \\
\hline FA-PO-150-12-2 Linear Actuator & 60 & Firgelli Automation \\
\hline High Current DC Motor Driver & 0.08 & Firgelli Automation \\
\hline 667oz-in NEMA-17 Stepper Motor & 20.4 & Phidgets \\
\hline CZL635 20 $\mathrm{kg}$ Load Cell & 0.025 & Phidgets \\
\hline DRV8825 Stepper Motor Driver & 0.005 & Pololu \\
\hline HX711 Load Cell Amplifier & 0.008 & Sparkfun \\
\hline
\end{tabular}

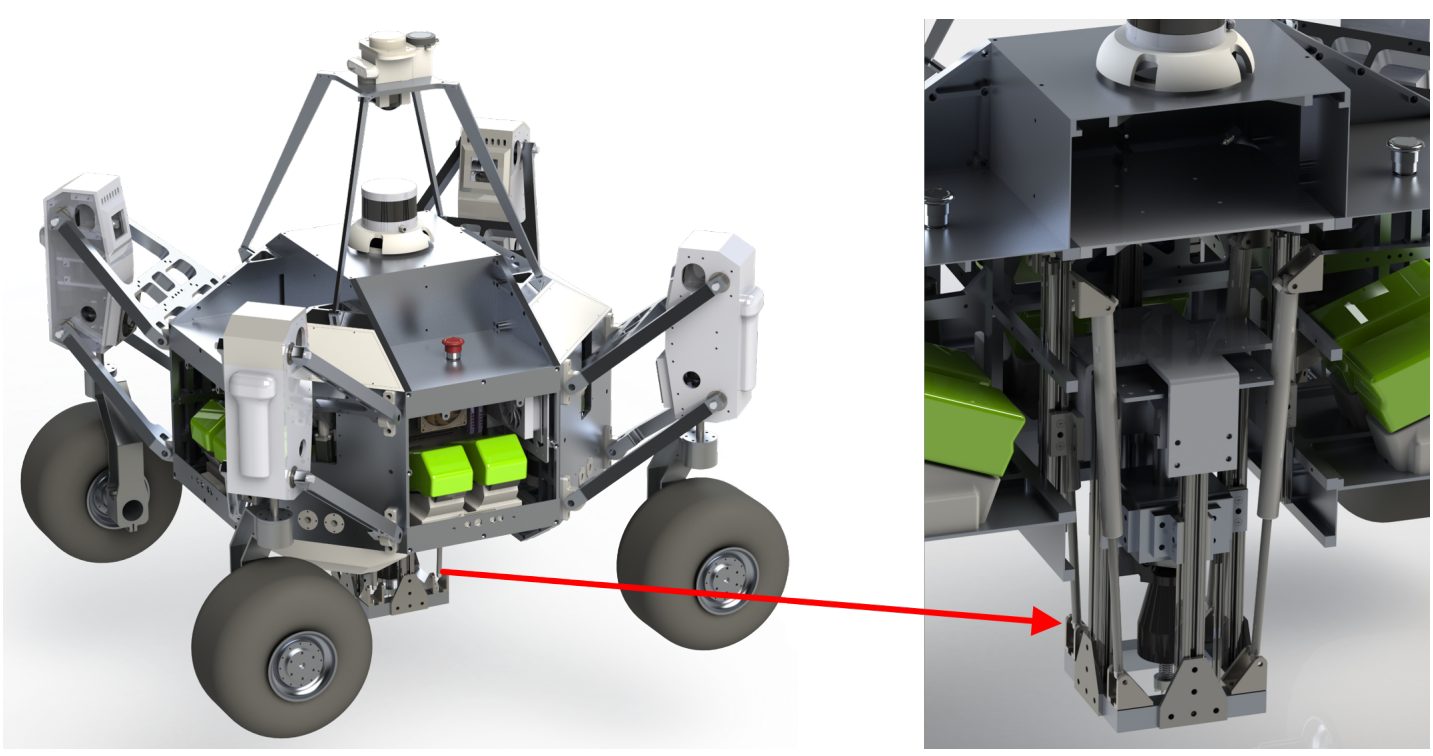

Figure 5.15: Mounted shear vane device inside Fast Traverse Rover (FTR). Credit: D. Covell.

- The noisy measurement is not sufficient to differentiate between two or more categories, at least one of them is dangerous and/or one of them would trigger a replanning event should it be the actual category the rover is about to traverse. The confusion happens when the probabilities of two or more terrains is between $25 \%$ and $75 \%$. This type of exact measurement trigger will be referred to as "confusion", but is often related to a potential dangerous terrain ahead as well (meaning, category D or $\mathrm{F}$ has a probability greater than $25 \%$ but lower than $75 \%)$.

Once the exact measurement is performed, the results are analyzed to retrieve $c$ and $\phi$, from which the traversability class is identified. Once known, it is paired with CFA and slope to obtain the corresponding velocity category. For Mars, the range of expected values for intrinsic parameters corresponding to each traversability class is presented in Table 5.7. They are estimated after the 
Table 5.7: Parameters associated with each traversability class on soft terrains. Values are retrieved from the survey presented in Sullivan et al. (2011).

\begin{tabular}{|c|c|c|c|c|c|}
\hline Traversability class & 1 & 2 & 3 & 4 & 5 \\
\hline \hline Cohesion $(\mathrm{kPa})$ & $3 \pm 3$ & $4 \pm 2$ & 5.5 & 0 & 0 \\
\hline \hline Angle of internal friction $\left(^{\circ}\right)$ & $34 \pm 3$ & $37 \pm 1$ & 30 & $30-41$ & $30-41$ \\
\hline
\end{tabular}

study performed in Sullivan et al. (2011) that lists various Martian terrain properties derived from terramechanics experiments. 


\section{CHAPTER 6 Results}

This chapter explores the results of slip and speed prediction as presented in the previous chapter, as well as the path planning algorithm with integrated terrain monitoring and map update. It also presents testing results of the pocket shear vane, hand-held and mounted.

\subsection{Map of traversability}

\subsubsection{Velocity prediction}

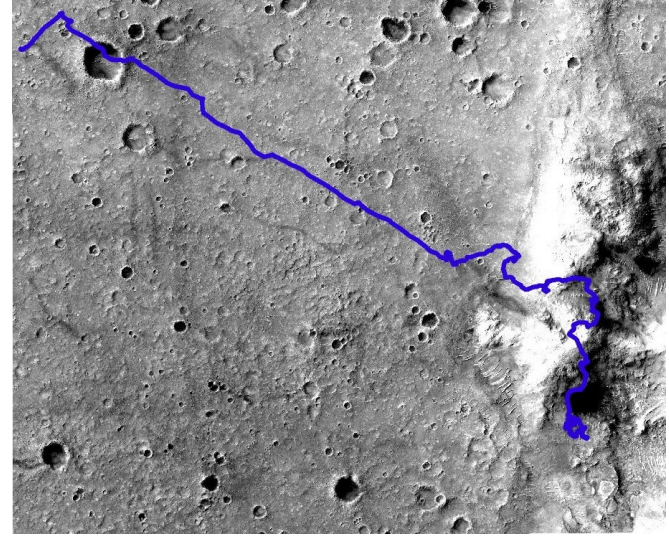

(a) Spirit's traverse on HiRISE image.

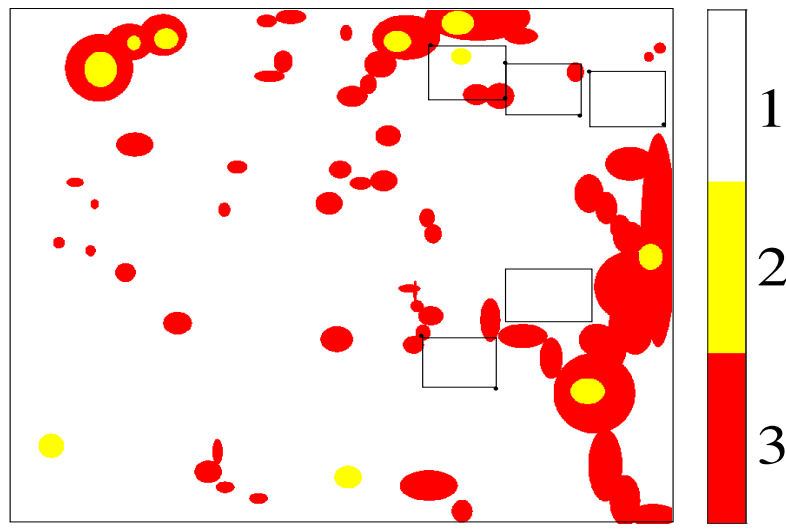

(c) CFA (1-3) map

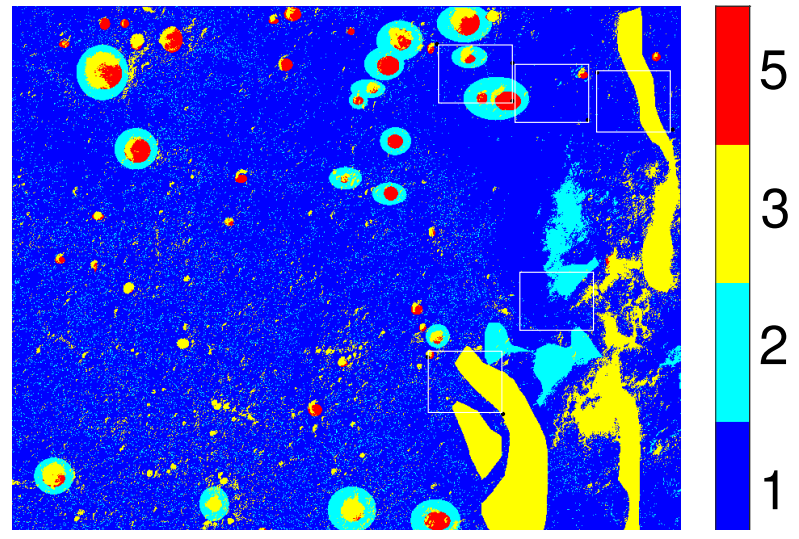

(b) Traversability class (1-5) map.

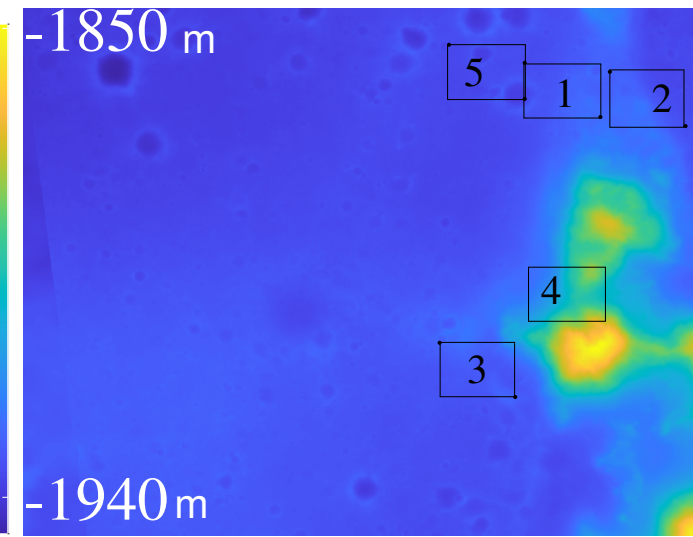

(d) DTM and trial numbers.

Figure 6.1: (a) HiRISE image (PSP 0017771650 RED A 01) with Spirit's traverse. Spirit's traverse is shown in blue. (b) traversability classes $(1,2,3,5)$. (c) CFA map: 1, low CFA $(<7 \%)$, 2, medium CFA (between $7 \%$ and $15 \%)$ and 3 , high CFA $(>15 \%)$. (d) DTM with subset maps numbered according to each trial performed (see Table 6.1 in section 5.3). The maps are $2915 \mathrm{~m}$ by $3540 \mathrm{~m}$ and the subset maps measure $301 \mathrm{~m}$ by $401 \mathrm{~m}$. 
For this section, the manually interpreted maps shown in section 5.2.2 are used. The method implemented to generate them led to each data set having the same resolution of $1 \mathrm{~m} / \mathrm{p} x$ and being already georeferenced, since they were built from the same basemap. Therefore, each pixel on the velocity gridmap was easily computed with values of slope, CFA and traversability class of the corresponding pixels. Each map is shown in Fig.6.1 and compared with the HiRISE image of the chosen area (Gusev Crater), with Fig.6.1a having a resolution of $25 \mathrm{~cm} / p x$ and Fig.6.1b, 6.1c and $6.1 \mathrm{~d}$ having a resolution of $1 \mathrm{~m} / \mathrm{px}$. The squares correspond to the subset maps used to test the path planning approach presented in section 5.3. An example of the final product is shown in Fig.6.2 and corresponds to the square labeled 1.

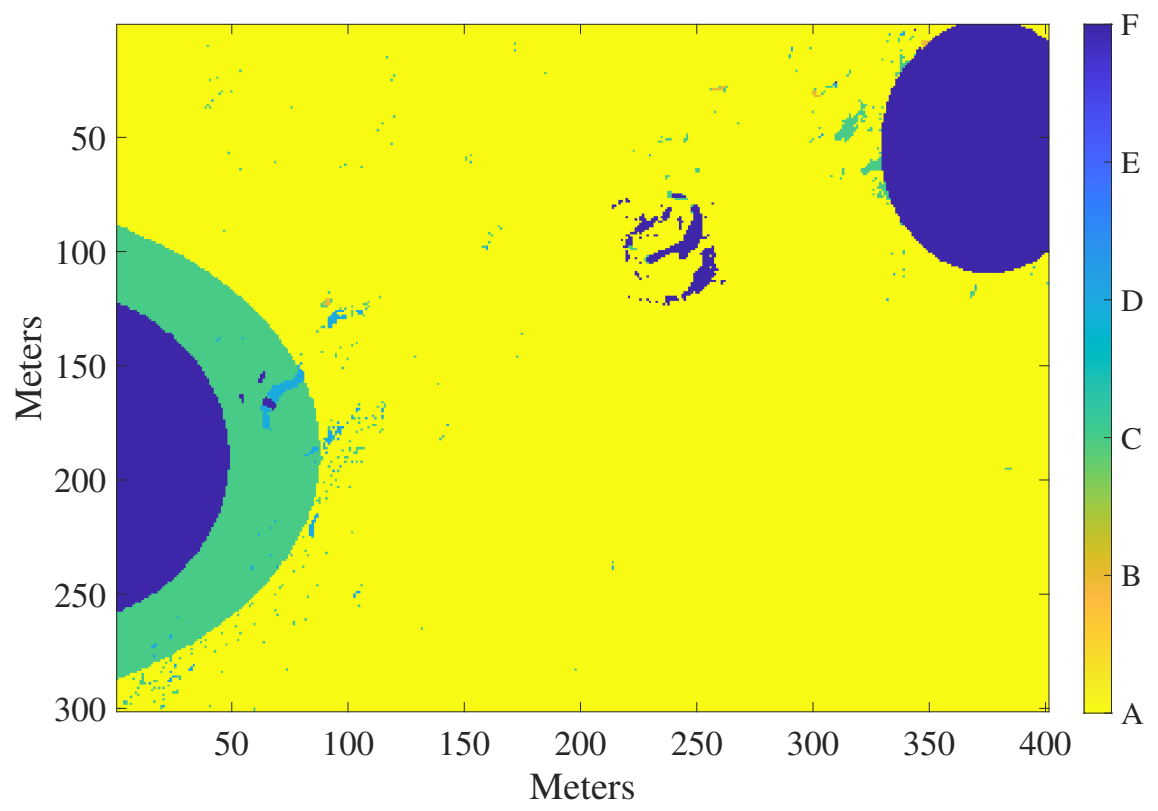

Figure 6.2: Example map of velocity categories (subset of the Columbia Hills area shown in Fig.6.1). This example is labeled 1 on Fig.6.1d.

\subsubsection{Slip prediction}

\subsubsection{Classifier training}

As a result of the PCA analysis in section 5.2.4.1, the classifier is trained on the A set (i.e., Spirit's data) with the variables contributing the most to the explained variance, that is, thermal inertia and slope. The best results are obtained using a decision tree classifier [Olshen et al., 1984] with a RUSboost algorithm [Seiffert et al., 2008] and a penalty cost of 5 for "low" misclassified as "high" and 1.5 for "high" misclassified as "low". The classifier algorithm is first chosen based on overall performance and has the following characteristics: maximum number of split is 50 and the number of learners is set to 30 . The same model is then trained without thermal inertia as a predictor. The performance of the classifier is presented in Fig.6.3. The overall performance is $68.7 \%$ for the classifier with two predictors and drops to $49.6 \%$ when using only slope as a predictor. Low slip 


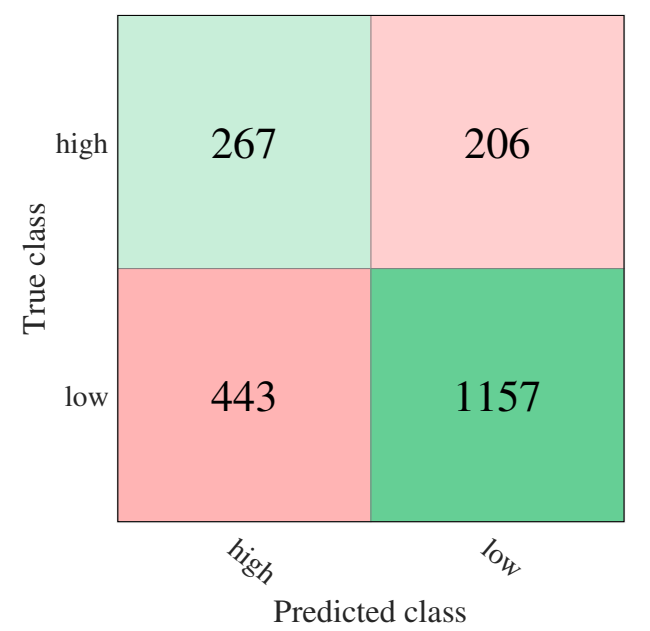

(a) Number of observation (2 predictors)

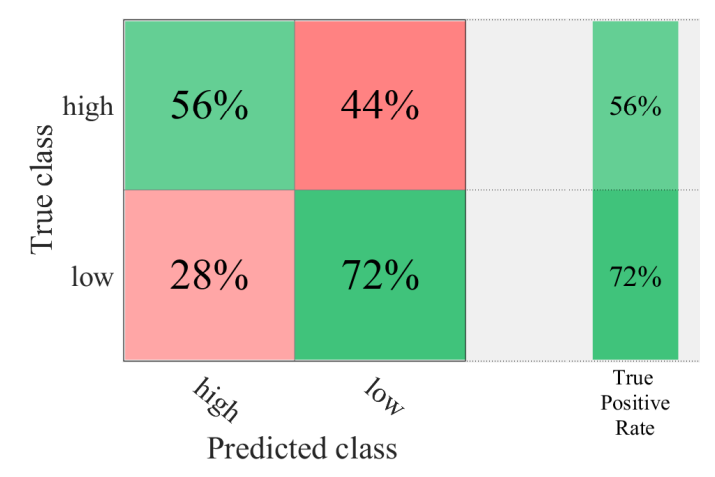

(c) True positive rate (2 predictors).

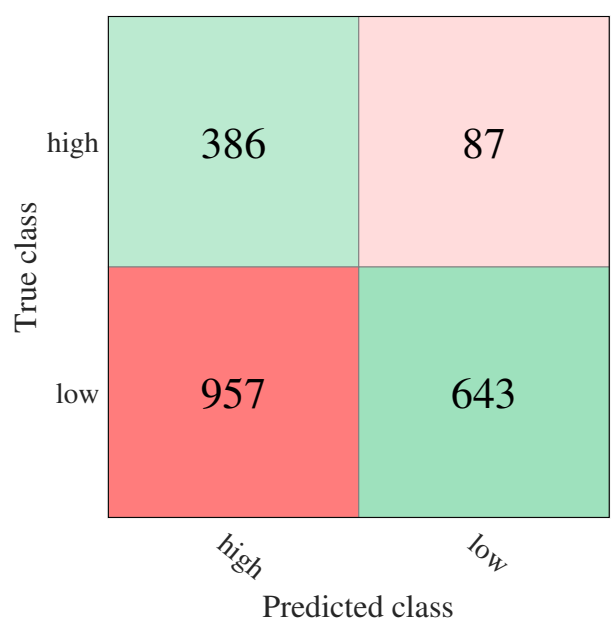

(b) Number of observations (1 predictor)

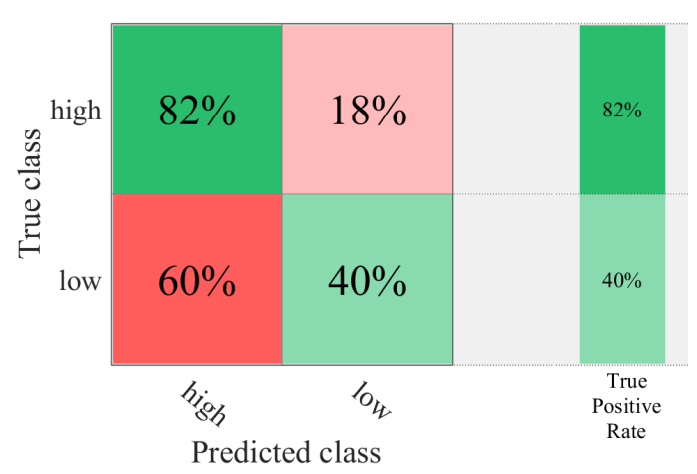

(d) True positive rate (1 predictor).

Figure 6.3: Confusion matrices showing the performance of the trained model on data set B2 for (a) thermal inertia and slope as predictors and (b) slope only as predictor.

prediction increases from $40 \%$ to $72 \%$ when adding thermal inertia; however, high slip prediction shows better results when the algorithm is trained with one predictor only. Overall, the conclusion tends to confirm the PCA analysis that thermal data are an important component of slip prediction analysis.

\subsubsection{Validation on new data}

All data from the B set are used to test the trained classifier. The results are presented in Fig.6.4, where the algorithm using thermal inertia and slope as predictors (Fig.6.5a) and the model taking only slope as predictor (Fig.6.5b) are tested.

The results show that the overall performance of the model is good (72.0\%) and decreases significantly (down to $56.3 \%$ ) when using only slope as a predictor. This confirms that thermal inertia is an important value to consider when predicting slip. The model is capable of predicting low slip and high slip pretty well, scoring $71.9 \%$ and $72.0 \%$ of the data, respectively. When thermal inertia is dropped and slope only is used, the model goes barely above average for low slip data, 


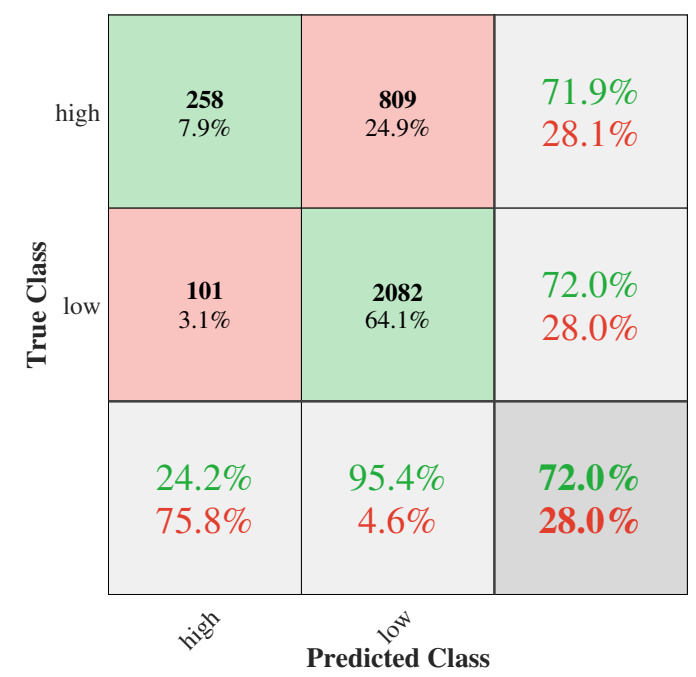

(a) Confusion matrix for test data using 2 predictors (thermal inertia and slope)

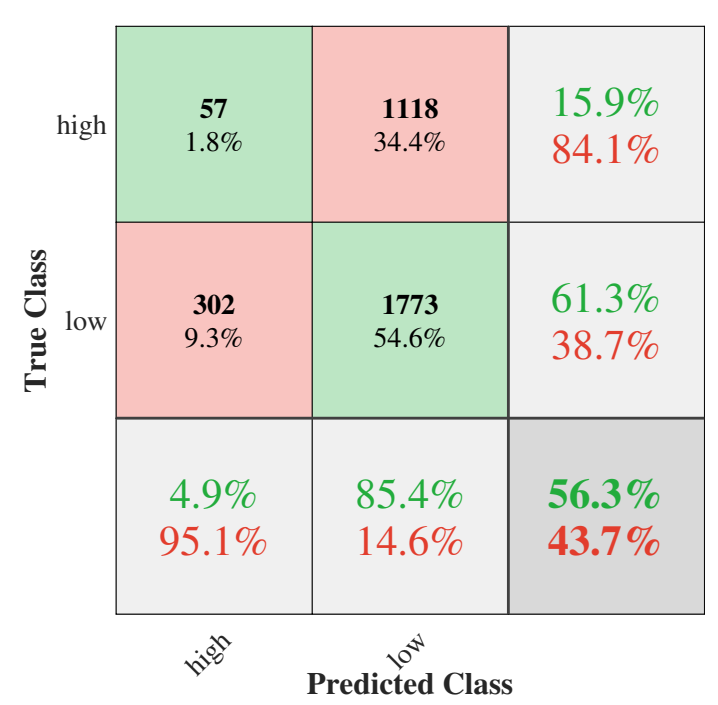

(b) Confusion matrix for test data using 1 predictor (slope)

Figure 6.4: Confusion matrices on test data sets A and B1 for (a) model with 2 predictors and (b) model with 1 predictor. Cells in green shows the correct prediction whereas red cells shows the errors from the classifier. The overall performance is displayed in the bottom right cell.

predicting only $61.3 \%$, and does not even correctly categorize more than a quarter of the high slip data, scoring only $15.9 \%$. The model trained with only slope as predictor therefore performs poorly and these results reinforce previous conclusions: thermal inertia is a valuable asset to add to the prediction of mobility performance.

\subsection{Terrain-aware path planning}

To test the performance of the path planning algorithm, small portions of the end product from the maps in Fig.6.1 are selected, chosen so that they cover the distance traversable in one sol (i.e., taking no more than eight hours), and are $301 \mathrm{~m}$ x $401 \mathrm{~m}$. Five simulations are performed on five different maps (see locations on Fig.6.1), to demonstrate that the algorithm give valid results independently of the location. These simulations have been run on the manually interpreted maps, which are very noisy on the south and west of the chosen area. More specifically, because of the grey value cutoff for classes 1 and 2, the lower left part of the map displayed unrealistic alternation of terrain on neighboring pixels (see section 5.2.3). Therefore, tests are run on the north and east parts of the map, where the noise was negligible. The start and goal locations are generated randomly with the constraints that the time it takes the rover to go from one to the other is no more than eight hours. Noisy measurements are performed at every nodes, and the tree depth was set to four nodes for all trials. As previously mentioned in section 5.4.3, when an exact measurement is performed, it is for one of the two following reasons: dangerous terrain ahead ("danger") or confusion between terrain ("confusion"). 


\subsubsection{Measurements and cost calculation}

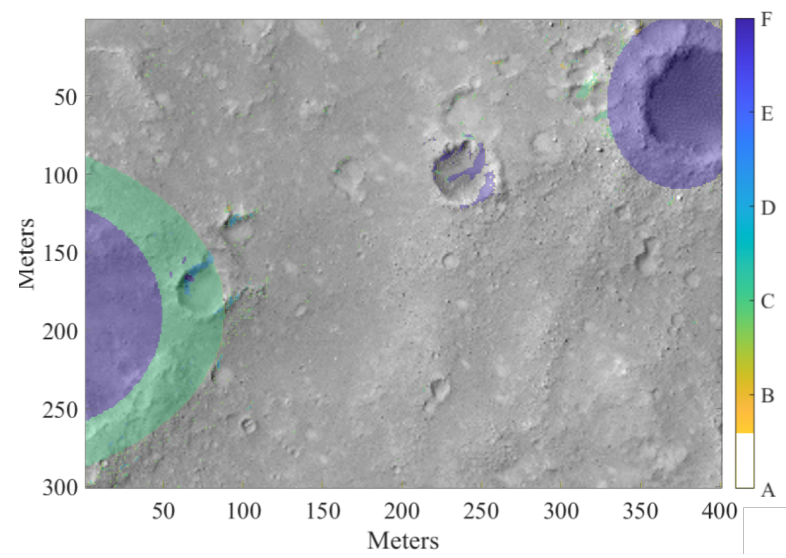

(a) Map of expected velocity categories.

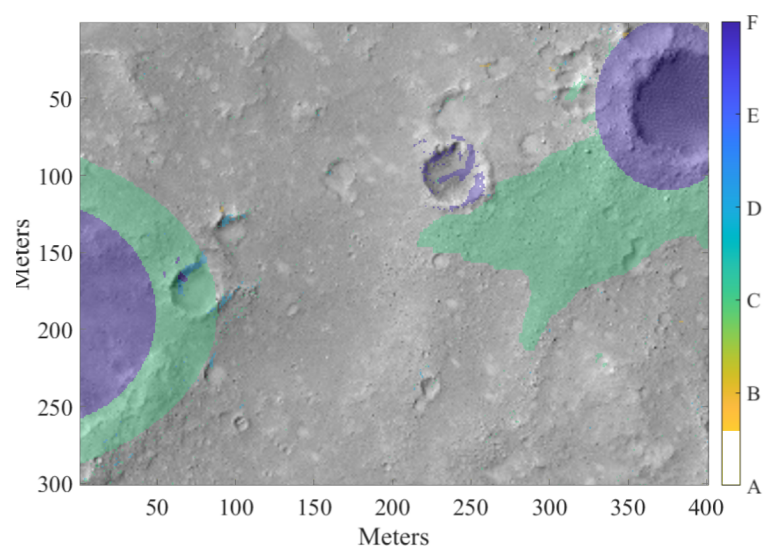

(b) Map of actual velocity categories.

Figure 6.5: Comparison of (a) expected velocity categories at the Columbia Hills and (b) actual velocity categories based on further analysis of HiRISE images at this specific location. The image shows that there could be a rougher terrain near the craters, reflected in the "truth" map in (b).

For simulations purposes, measurements are sampled from the "truth" map as mentioned in subsection 5.3.3, using the following methods:

- For noisy measurements, values for slip and speed are sampled from the distributions presented in subsection 5.3.2.2 according to the categories along the path shown on the "truth" map. For example, if the path is crossing category $\mathrm{C}$, which is initially believed to be A, then the slip and speed the rover experienced are sampled from $C$, even though the initial map shows category A. To smooth the transition from one value to another, the ranges from which slip and speed are sampled are made more narrow than what the actual PDFs would normally give. Moreover, the slip prediction classifier is run to determine whether slip will be more or less than $30 \%$. The resulting numbers are then analyzed individually to ensure a smooth transition from one to the other. This means that if the rover goes at a speed of $30 \mathrm{~km} / \mathrm{hr}$, then the previous speeds as well as the following need to be reasonably close to this value. Indeed, it is unlikely that the rover will go from $5 \mathrm{~m} / \mathrm{hr}$ to $30 \mathrm{~m} / \mathrm{hr}$ or 60 $\mathrm{m} / \mathrm{hr}$ (and vice versa) in a couple of meters. It is more likely that the rover will accelerate or decelerate gradually, over 15 or $20 \mathrm{~m}$. Abrupt changes led, in fact, to impossible results from the simulations. Moreover, the slip prediction classifier was run over each map used in the trials and slip was sampled according to the results, which showed that it was expected to be mostly less than $30 \%$ on all of the trial maps.

- For exact measurements, categories are directly sampled from the "truth" map, i.e., if the rover is crossing $\mathrm{C}$, then the exact measurement will return category $\mathrm{C}$ with $100 \%$ accuracy. Details on how the exact measurement can analyze a terrain to output the correct velocity category with low certainty is detailed in section 5.4 and results are shown in section 6.3.

An example of a "truth" map is presented in Fig.6.5 over the subset map number 1 (see Fig.6.1 for location). 
The costs calculated in the planning process correspond to the time it takes the rover to reach the goal, given its actual velocity from the noisy measurements generated, which have been derived from the "truth" map as mentioned above. When replanning occurs due to a change of category, the cost of the initial path, had the terrain not changed, is calculated in a similar manner, with the assumption of what velocity the rover would have experienced without a change of terrain and according to the geology given by the "truth" map. This cost is then compared to the cost of the new traverse that includes replanning (if applicable) and change of terrain. In other words, "noisy measurements" have been generated along each path, regardless of whether the rover traverses it, and used to compare costs of different paths and/or their combinations. The costs given do not take into account the time it takes to perform an exact measurement.

For all the trials, monitoring (a.k.a., noisy measurement) is performed at every node and the maximum length of a branch of the belief propagation tree is $N=4$ nodes (also known as tree depth).

\subsubsection{Trial one}

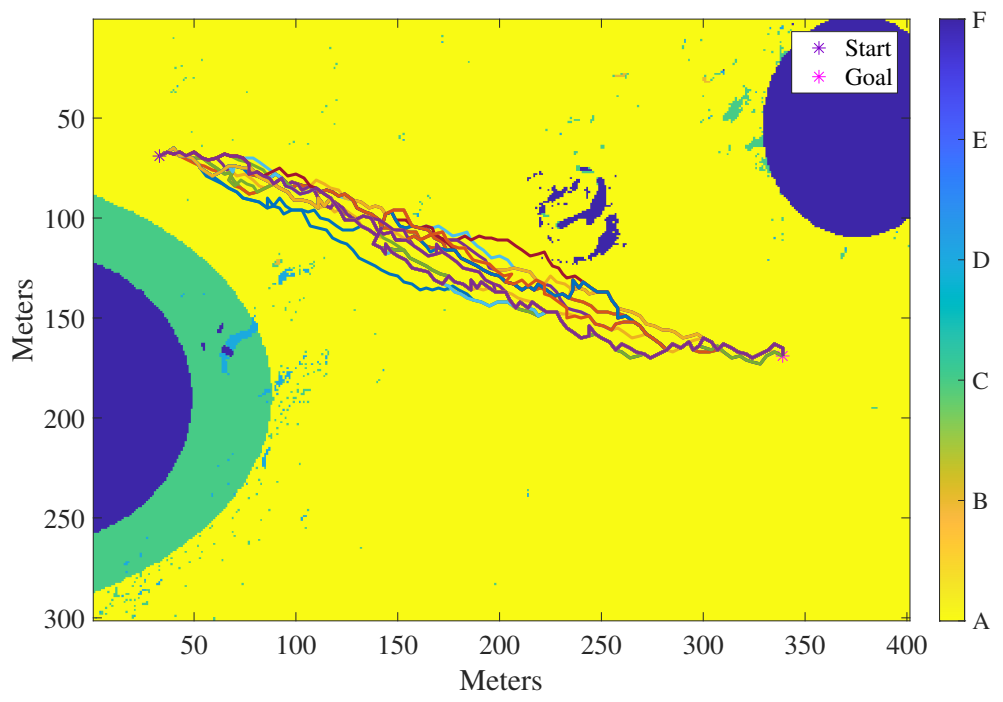

Figure 6.6: Inital map of velocity categories A to F, with 37 paths. Credit: Hedrick et al., 2020.

In this example, there are 37 paths computed within the allotted budget (costs are between 5.5 and 6.5 hours) with a path length between $354.5 \mathrm{~m}$ and $430.2 \mathrm{~m}$. The slip prediction classifier indicates that the rover should experience less than $30 \%$ slip everywhere in the area. Initially, all paths are traversing category A.

During the traverse, noisy measurements are performed and it appears that one of them led to detecting a dangerous terrain ahead, which in turn led to performing an exact measurement. It was determined, through propagation of this new information acquired, that there was no danger ahead. Later, following an actual change of terrain detected (leading to a change of velocity category from A to $\mathrm{C}$ ), the rover finds that the initial path chosen is no longer the fastest. By propagating this new information ahead, as seen with the belief propagation tree in Fig.6.8, it gains sufficient knowledge 


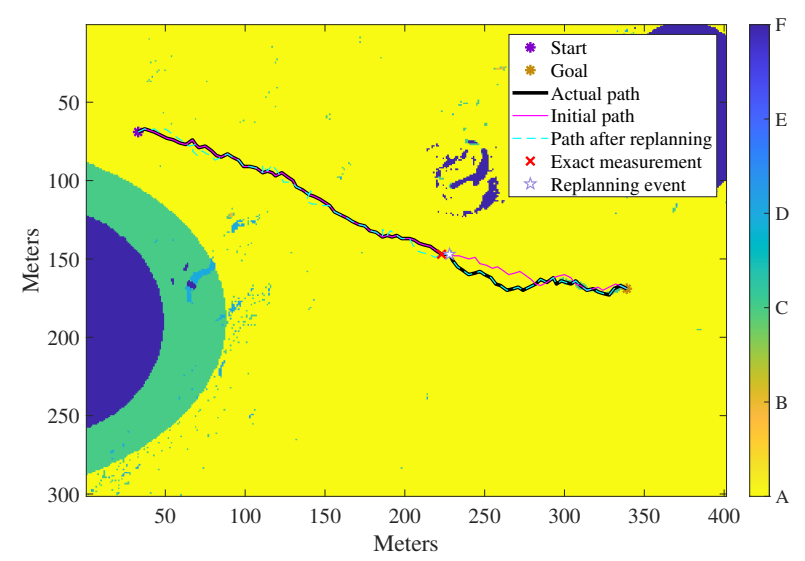

(a) Actual path and its components shown on the locally updated map.

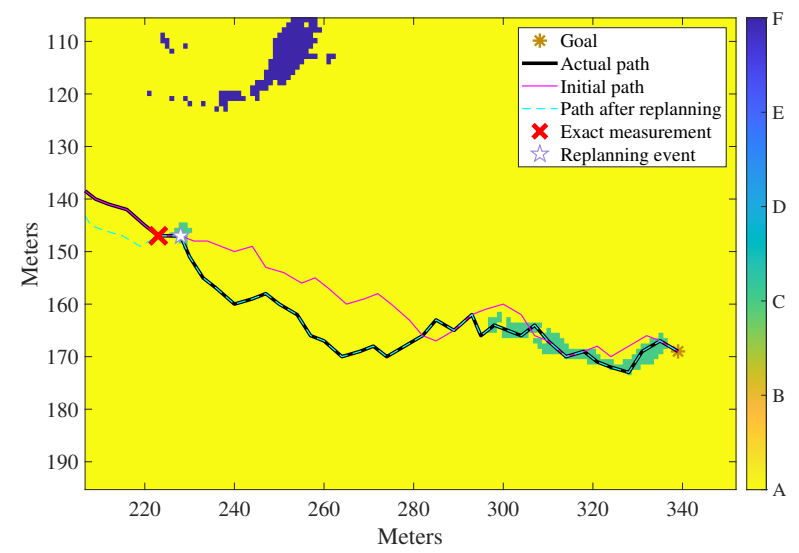

(c) Actual path and its components shown on updated map with a focus on the exact measurement, replanning and local update.

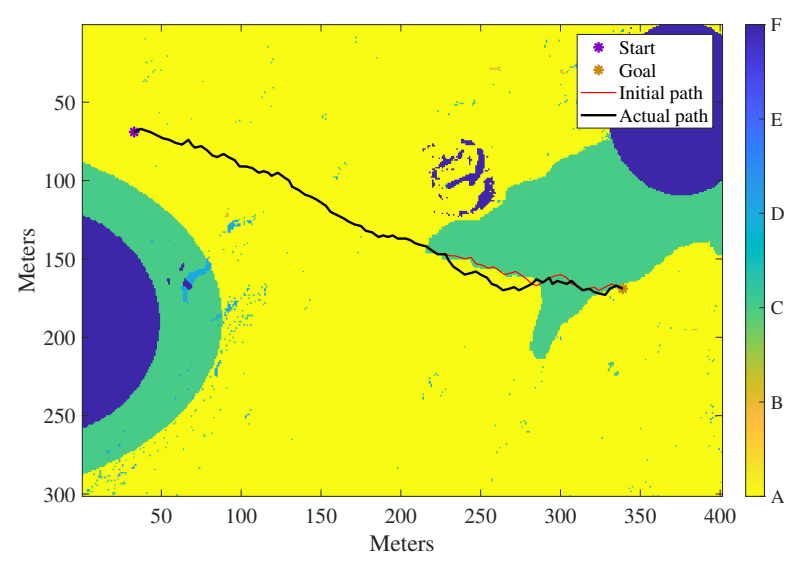

(b) Actual path on "truth" map from which measurements are sampled.

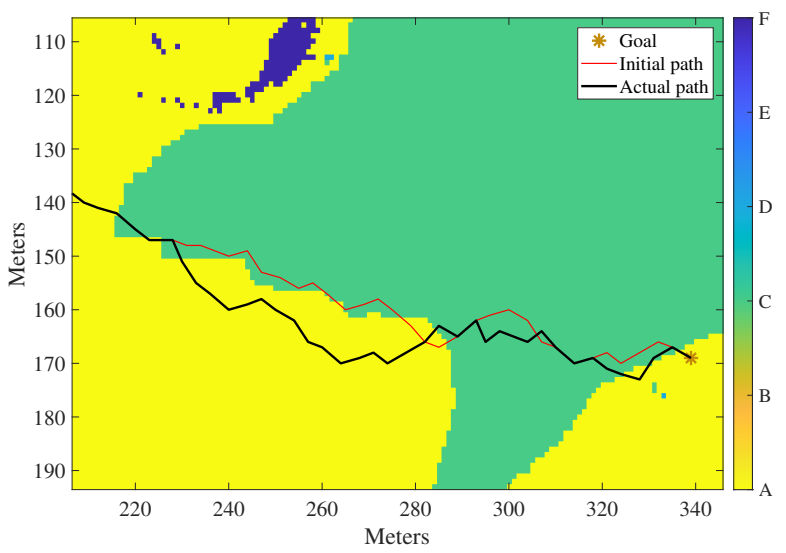

(d) Actual path shown on the "truth" map from which the measurements are sampled, showing the accuracy of the update on a local scale.

Figure 6.7: Trial 1: actual path on (a) updated map and (b) "truth" map. One exact measurement is performed due to potential dangerous terrain ahead. One replanning event occurs in the traverse, saving about seven minutes.

to make a decision regarding its traverse and chooses a faster and safer path. A different option is indeed found that minimizes the remaining time to the goal by avoiding the terrain in category $\mathrm{C}$ for as long as possible (Fig.6.7), thus gaining about seven minutes. A close-up view of the exact measurement followed by a replanning event is shown in Fig.6.7c.

This example shows that the diversity of the paths is needed to offer less costly alternatives from the current route, and that having overlapping segments is necessary to allow for quick deviations from the original path. The rover gets to its goal in five hours and 39 minutes in the simulation, and would have taken five hours and 46 minutes along the initial route. It also demonstrates that noisy measurements can be enough to make replanning decisions when a more difficult terrain is found along the initial route. Even if the time saved from replanning is not significant (only about seven minutes), choosing to cross a lower category (A instead of $\mathrm{C}$ ) means that the rover decides on a safer path as well as (slightly) faster. Indeed, by definition, category $\mathrm{C}$ carries more risk than category A, due to potentially rougher terrains being traversed. 


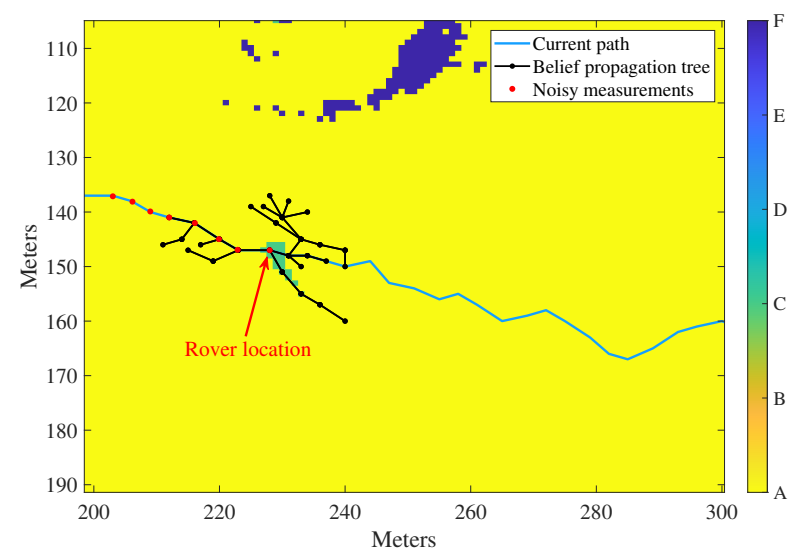

(a) Belief propagation tree detecting a more difficult terrain ahead, leading to a replanning event.

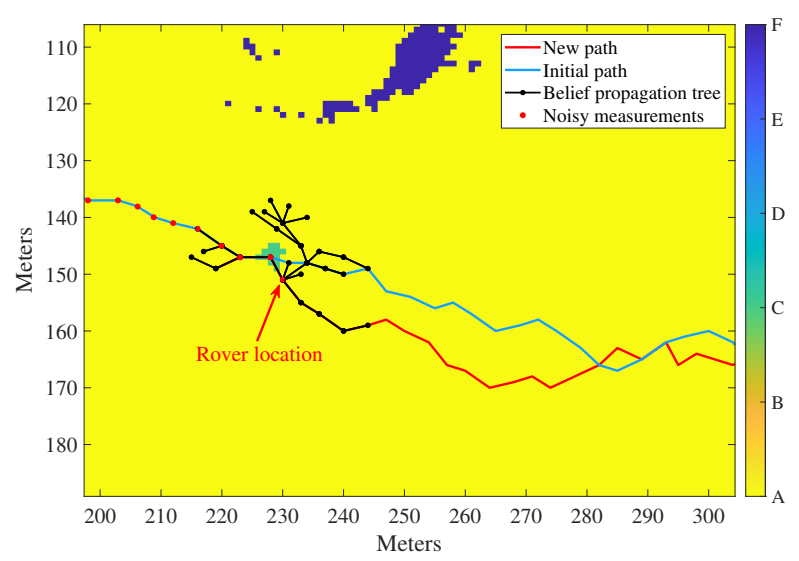

(b) Belief propagation tree updating the map locally, after a replanning event.

Figure 6.8: Belief propagation tree passing the message of a newly detected category $\mathrm{C}$, which is more challenging than A. The message passing leads to the rover making the decision to choose a faster path south of its original traverse, that would cross less of the more difficult terrain.

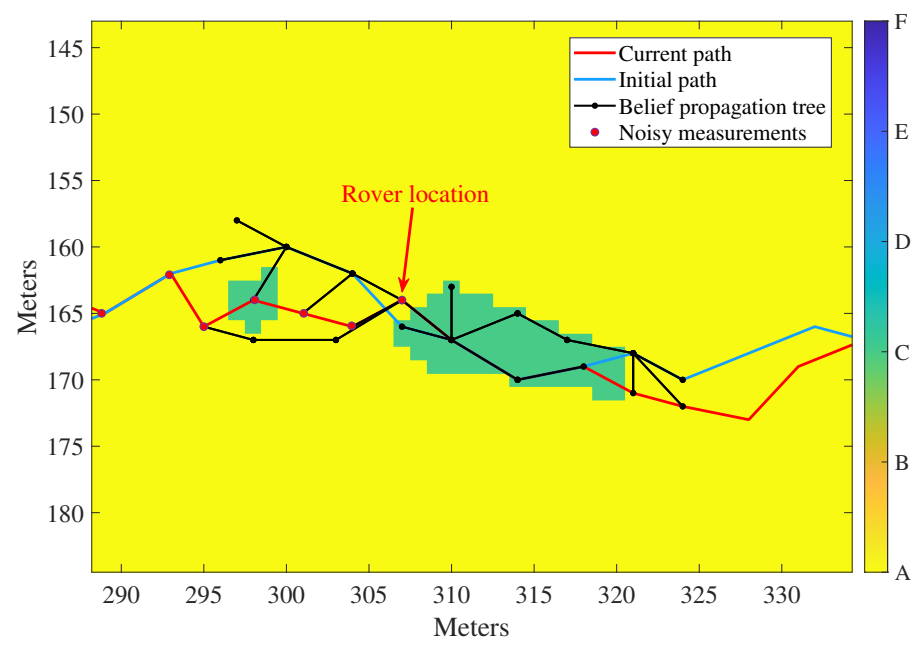

Figure 6.9: Belief propagation tree with noisy measurement giving category A (even though, in truth, it is located on a patch of category $\mathrm{C}$ ) but detecting accurately category $\mathrm{C}$ ahead.

\subsubsection{Trial two}

There are 70 options, with length varying from $311.5 \mathrm{~m}$ to $334.0 \mathrm{~m}$. The results are presented in Fig.6.10. The slip is predicted to be less than 30\% along all the options, with the exception of the tip of each one (about 5 nodes) that shows slip higher than $30 \%$.

The rover continues along its initial path until it reaches its goal. Based on noisy measurements only, it detects that the map is inaccurate and that it is traversing category $\mathrm{C}$ instead of the expected D (see close-up view on Fig.6.10c). 


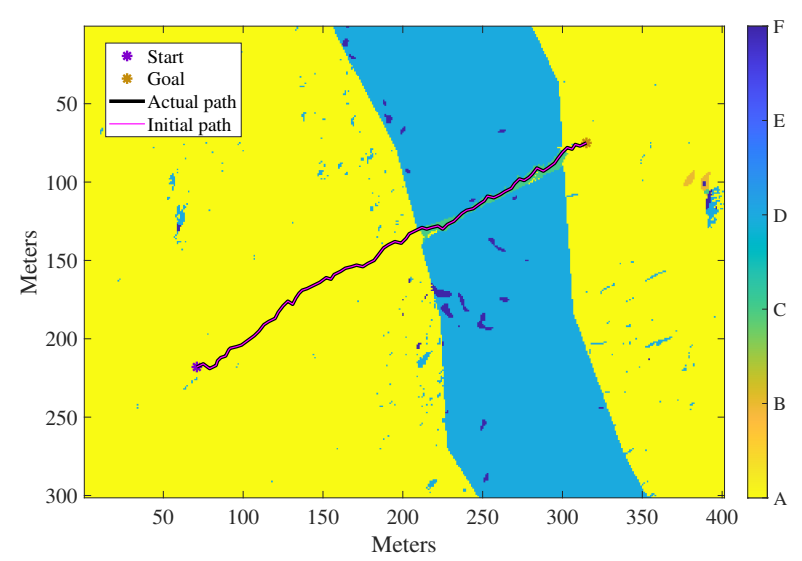

(a) Actual path (equivalent to initial path) shown on the updated map.

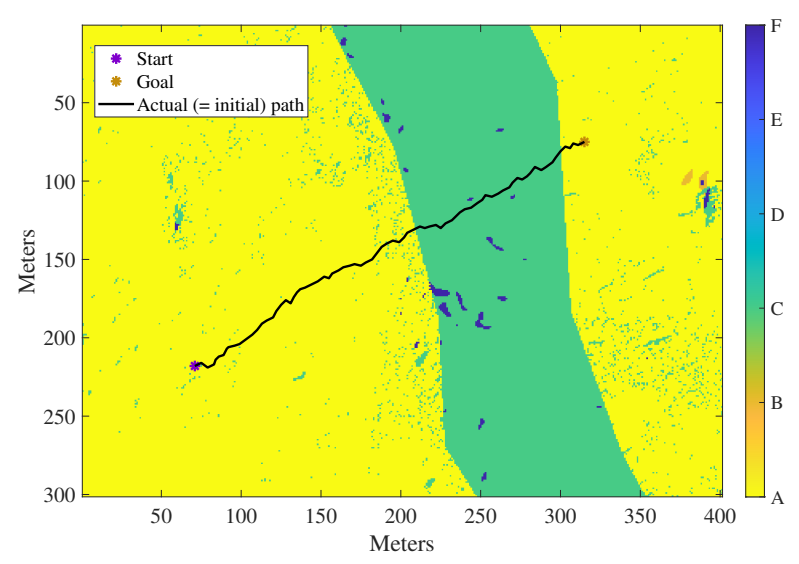

(b) Actual path on "truth" map from which measurements are sampled.

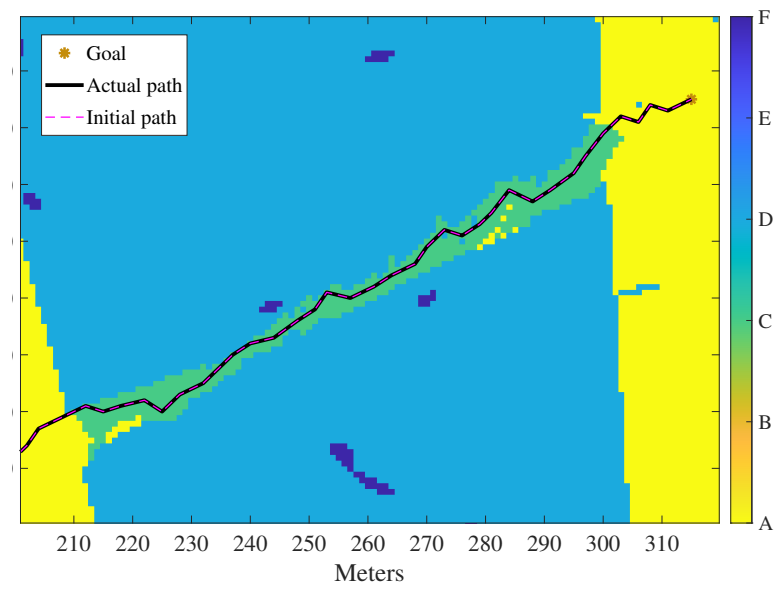

(c) Zoom on the area updated by belief propagation after measurements.

Figure 6.10: Trial 2: actual path (equivalent to initial path) on (a) updated map and (b) "truth" map. There is no replanning necessary, even after an exact measurement is performed due to potential dangerous terrain detected ahead.

\subsubsection{Trial three}

This trial has the shortest completion time of all, and features 26 paths, covering distances between $123 \mathrm{~m}$ and $138 \mathrm{~m}$, each covering areas that should trigger less than $30 \%$ slip according to the slip prediction classifier. One replanning event happens to avoid more difficult terrain. The traverse and its components are shown in Fig.6.11, with a close-up view of the area where the replanning event took place. The rover completes its traverse in two hours four minutes, instead of two hours and seven minutes, had it followed the initial route. If the time saved from replanning is not significant (only about three minutes), choosing to cross a lower category (A instead of C) means that the rover chooses a safer path, as well as a (slightly) faster one. As in trial one (see subsection 6.2.2), even if the time saved is not significant (three and a half minutes), the replanning goes beyond saving time only, by choosing safer categories to traverse. 


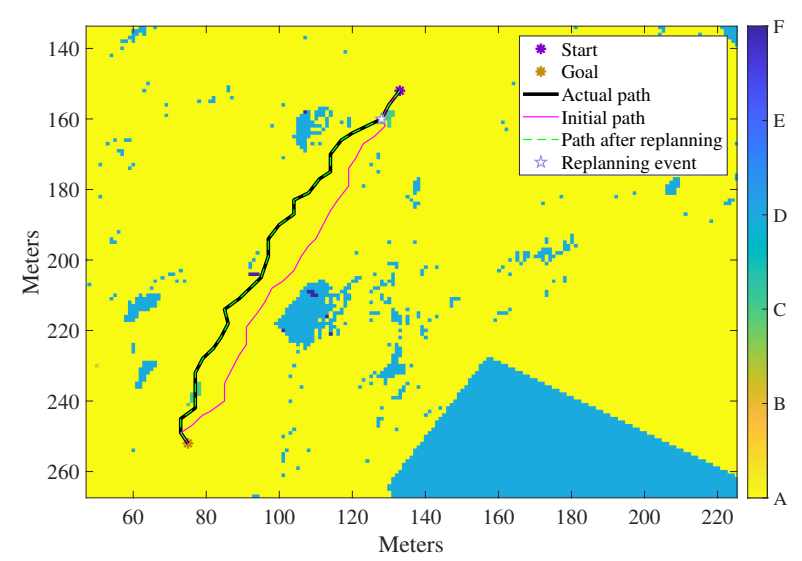

(a) Actual path and its components shown on the updated map.

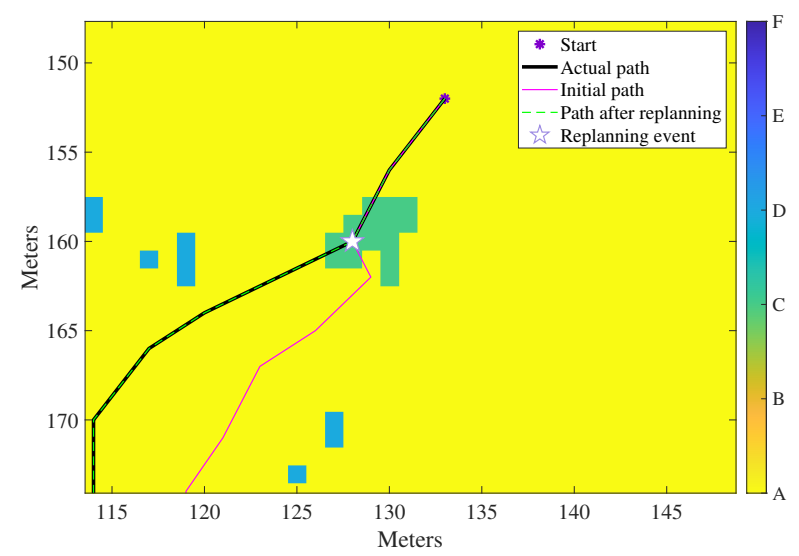

(c) Close view of the actual path and its components shown on the updated map.

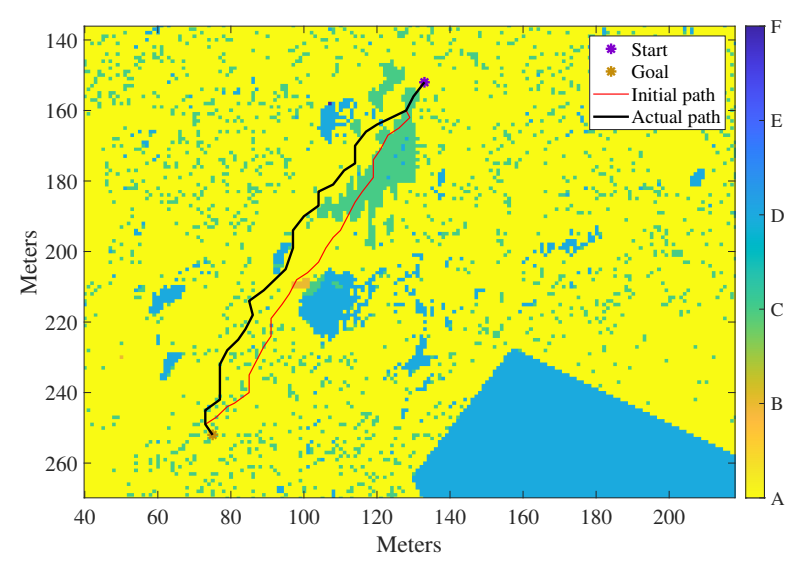

(b) Actual path on "truth" map from which measurements are sampled.

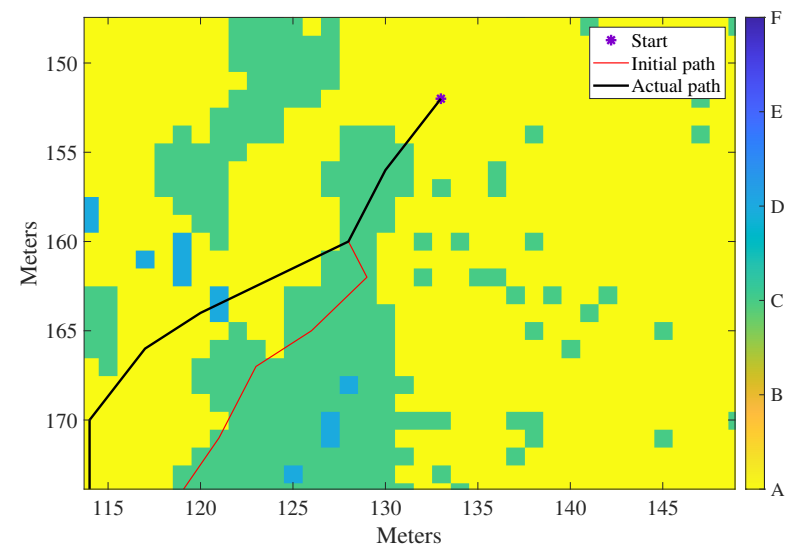

(d) Close view of the actual path on the "truth" map, showing the challenges of its initial route.

Figure 6.11: Trial 3: actual path on (a) updated map and (b) "truth" map. One replanning event is performed to avoid more difficult terrain.

\subsubsection{Trial four}

In this example, there are 36 paths available, covering distances between $420 \mathrm{~m}$ and $428 \mathrm{~m}$, with slip predicted to be less than $30 \%$ all along, according to the classifier. One exact measurement was performed due to a confusion of terrains (B and D) detected, and the outcome determined that the terrain was simply category B (see Fig.6.12). Fig.6.12e shows that the area is not correctly captured by the local update, even with the exact measurement identifying category B. The "truth" category is correctly shown on the pixel the measurement is taken, but the algorithm fails to adequately update the end leaves of the belief propagation tree. However, it updates the map to show a more difficult area than it actually is, which is a better alternative and means that the rover is actually crossing safer terrain than expected. Similarly to what is explained in subsection 6.2.2 about the possible confusion between $\mathrm{A}$ and $\mathrm{C}$, given how close $\mathrm{B}$ and $\mathrm{C}$ are, the confusion in the update is not unreasonable, nor is it detrimental to the rover and the planning process. 


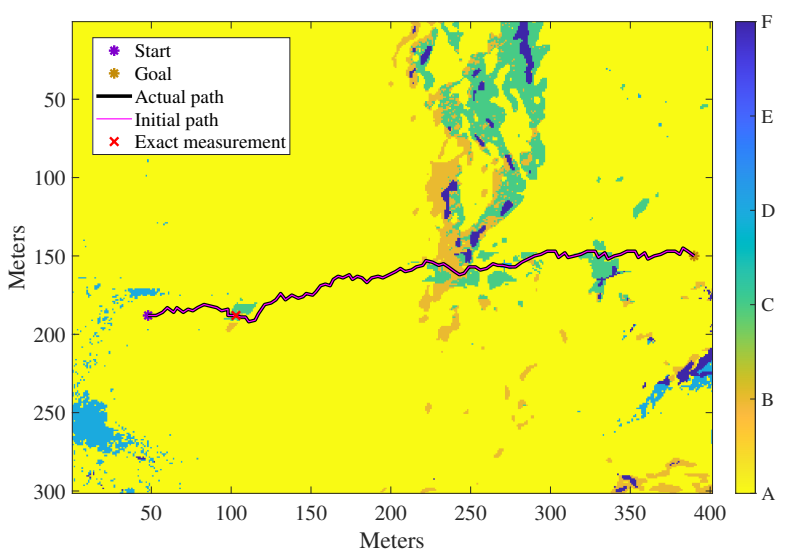

(a) Actual (initial) path shown on the updated map.

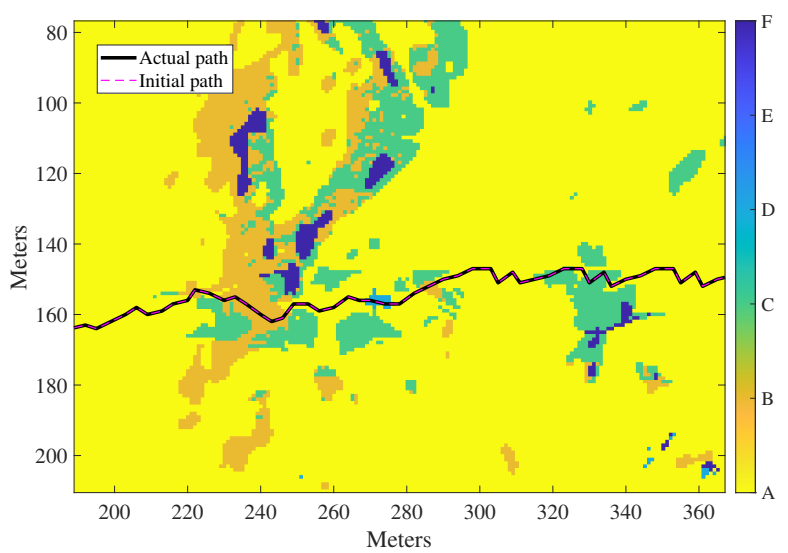

(c) Close view of the path on locally updated map.

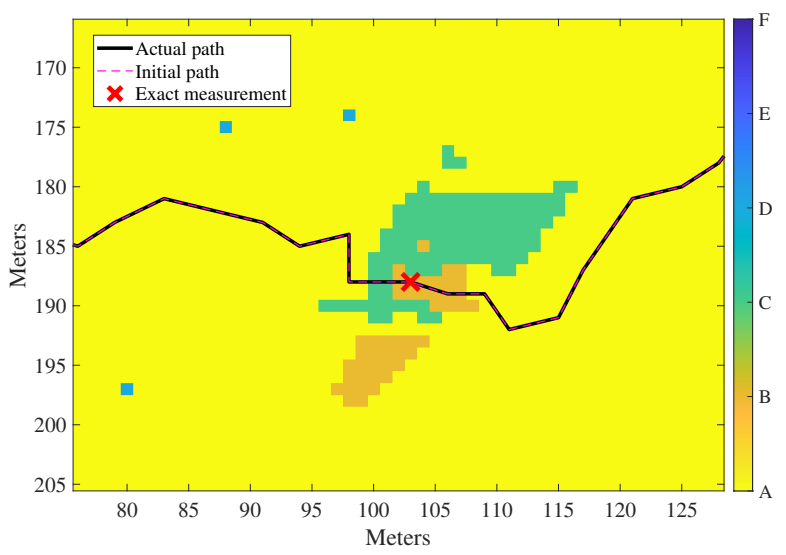

(e) Exact measurement site (due to confusion).

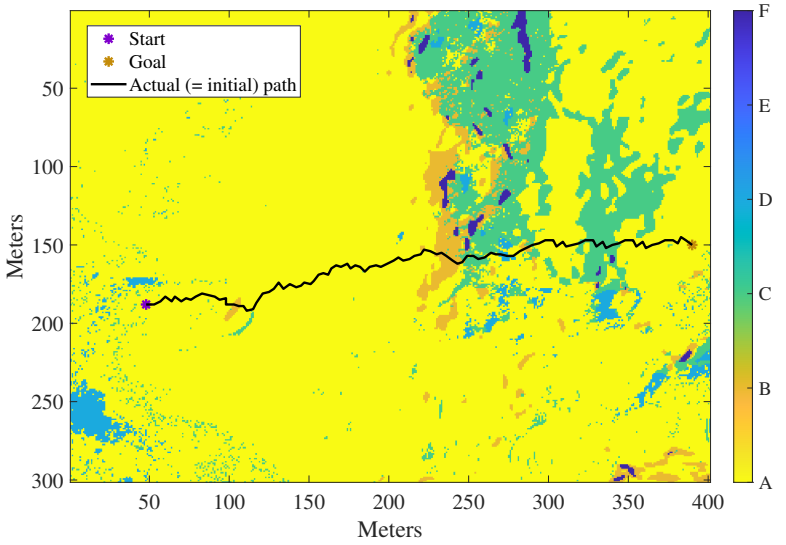

(b) Actual path (initial path) shown on "truth" map.

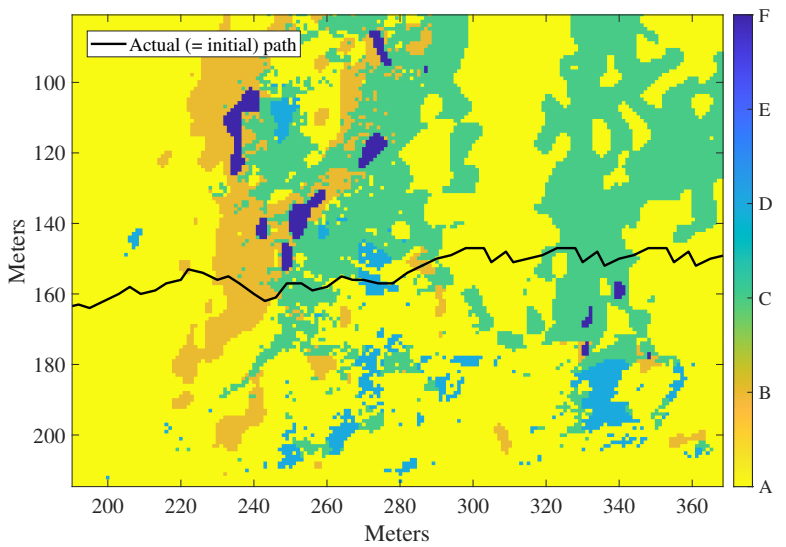

(d) Close view of the actual path on "truth" map.

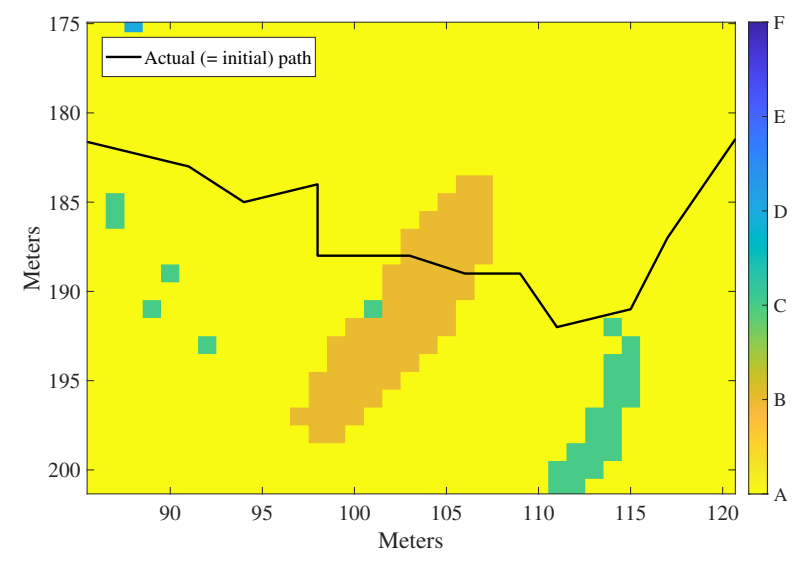

(f) “Truth" category at the exact measurement site.

Figure 6.12: Trial 4: actual path on (a) updated map and (b) "truth" map . 


\subsubsection{Trial five}

This trial shows 65 paths covering distances between $269 \mathrm{~m}$ and $297 \mathrm{~m}$ and the classifier predicts less than $30 \%$ slip everywhere. The situation shown in this trial is more challenging that previous ones, because the rover is near a crater. Initially, the crater is thought to be surrounded by category C, where the "truth" map actually shows category D. Upon detecting that the rover is in fact on a more dangerous terrain, it replans its traverse as many times as necessary to go through areas that are less challenging, as shown in Fig.6.13. It shows on the "truth" map that the rover eventually follows the safer terrain for as long as possible (Fig.6.13d), and is able to do so by jumping from one pre-planned option to another (Fig.6.13c). The rover gains about two and a half minutes and more goes intly,mportathrough areas that present less of a danger to the rover.

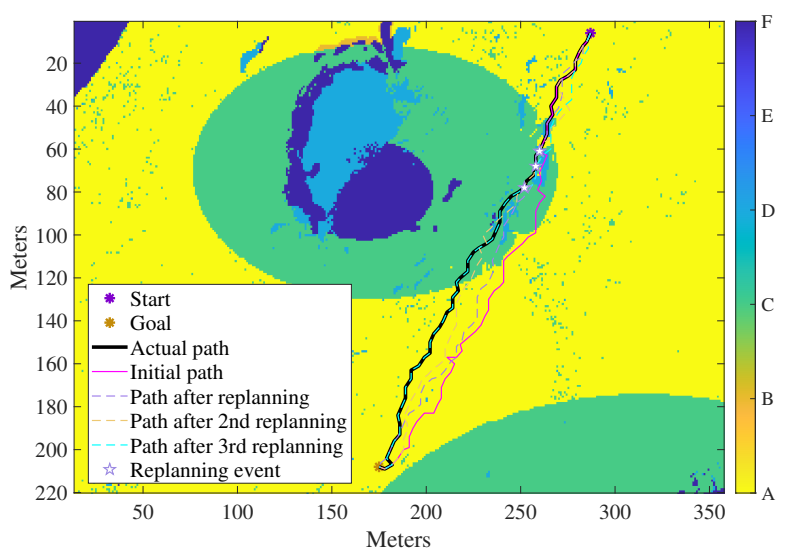

(a) Actual path and its components shown on the updated map.

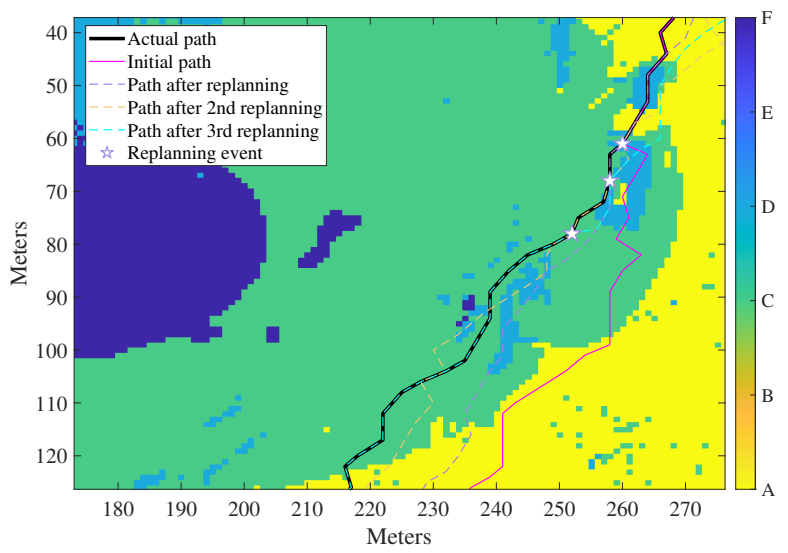

(c) Close view of the replanning nodes.

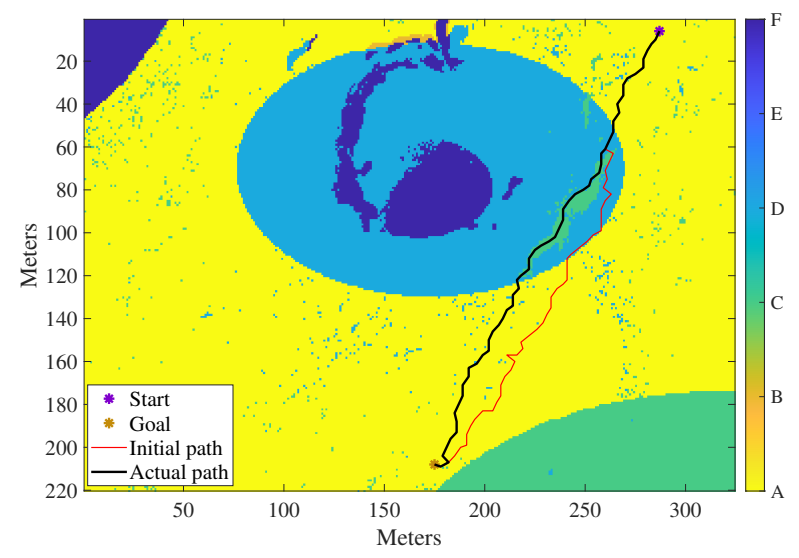

(b) Actual path on "truth" map from which measurements are sampled.

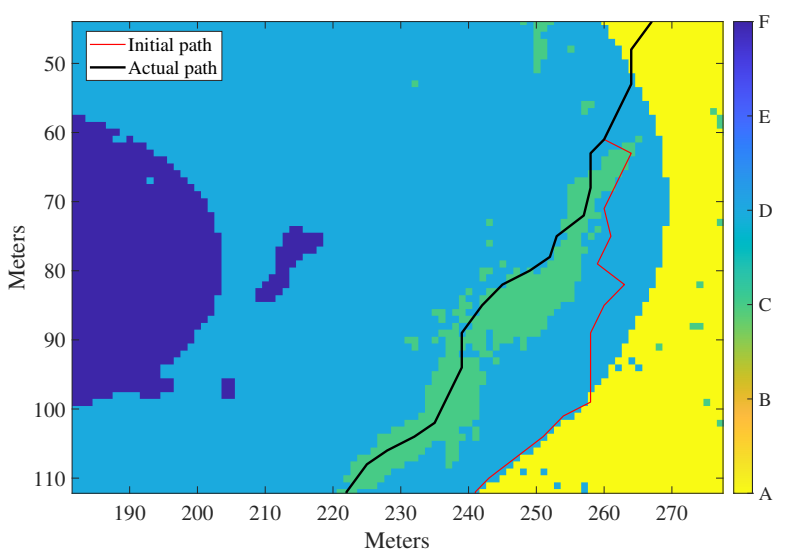

(d) Close view of the actual path on the "truth" map.

Figure 6.13: Trial 5: actual path on (a) updated map and (b) "truth" map with three replanning events. The replanning leads to following a safer terrain $(C$ instead of $D)$.

\subsubsection{Summary of trials and discussion}

The trials in Table 6.1 show that replanning is not always needed, nor are exact measurements (noisy measurements are, most of the time, sufficient for the rover). If the remaining time 
to the goal after an update is still the shortest along the current path, the rover maintains its course. They also demonstrate that replanning acts as a safety feature, by avoiding more dangerous terrain when possible and limiting the risks the rover could encounter. Moreover, these trials show that exact measurements are very seldomly acquired, which is an advantage considering the time and energy cost of deploying an instrument, as simple as it could be. The map update is not always accurate when further from the measured nodes (i.e., end of a tree branch), but it tends to update the cells to a more dangerous terrain than it actually is. There are several examples of this in trial 4 (see Fig.6.12) and trial 5 (see Fig.6.13). When outside the areas covered by the belief trees, the rover simply keeps the beliefs without updating them.

However, these results need to be put in the context of simulation. They were obtained by randomly sampling slip and speed along the path according to the true category from the "truth" map (see section 6.2.1), and real experienced slip combined with actual speed might lead to slightly different results, such as even less exact measurements or more replanning events.

Table 6.1: Trials 1 to 4 (see Fig.6.1 for location).

\begin{tabular}{|l||l|l|l|l|l|}
\hline Trials, distance start-goal & $1(344 \mathrm{~m})$ & $2(283 \mathrm{~m})$ & $3(116 \mathrm{~m})$ & $4(344 \mathrm{~m})$ & $5(231 \mathrm{~m})$ \\
\hline \hline Categories along path & A,C & A,C & A,C,D & A,B,C,D & A,D \\
\hline $\begin{array}{l}\text { Cost initial path (esti- } \\
\text { mated) }\end{array}$ & $5.55 \mathrm{hrs}$ & $7.39 \mathrm{hrs}$ & $1.96 \mathrm{hrs}$ & $4.45 \mathrm{hrs}$ & $5.11 \mathrm{hrs}$ \\
\hline Replanning events & 1 & 0 & 1 & 0 & 3 \\
\hline Actual cost of initial path & $5.77 \mathrm{hrs}$ & $5.74 \mathrm{hrs}$ & $2.12 \mathrm{hrs}$ & $7.25 \mathrm{hrs}$ & $5.56 \mathrm{hrs}$ \\
\hline Cost final path & $5.65 \mathrm{hrs}$ & $5.74 \mathrm{hrs}$ & $2.06 \mathrm{hrs}$ & $7.25 \mathrm{hrs}$ & $5.52 \mathrm{hrs}$ \\
\hline Time saved & $6.6 \mathrm{~min}$ & $\mathrm{~N} / \mathrm{A}$ & $3.6 \mathrm{~min}$ & $\mathrm{~N} / \mathrm{A}$ & $2.4 \mathrm{~min}$ \\
\hline Exact measurement & 1 & 0 & 0 & 1 & 0 \\
\hline $\begin{array}{l}\text { Reason for exact measure- } \\
\text { ment }\end{array}$ & Danger & N/A & N/A & $\begin{array}{l}\text { Confusion } \\
\text { (B,D) }\end{array}$ & N/A \\
\hline
\end{tabular}

\subsection{Instrumentation for in-situ soil analysis}

The modified pocket shear vane was tested in known soils (per United Soil Classification System, or USCS), in the laboratory and in the field, to verify that the modifications would lead to adequate results from which cohesion, angle of internal friction and bearing capacity could be retrieved.

\subsubsection{Hand-held pocket shear vane}

\subsubsection{Testing settings}

The hand-held modified pocket shear vane is tested in samples with known parameters (referred to as controlled samples), as well as in the field, in visually identified soils (referred to as in-situ). The controlled samples are the following (the official USCS label is specified for each one): 
- Clay of low plasticity (USCS CL)

- Silt loam, compacted (USCS ML, OL, MH, OH)

- Fine sand (USCS SW, SP)

The in-situ soils are the following:

- Silt loam, saturated (USCS ML, OL, MH, OH)

- Sand (USCS SW, SP)

Several tests are run for each load for each of the controlled samples (five per sample), whereas only one test per load is performed in-situ, to stay as close as possible to conditions on Mars (i.e., limited capability to perform tests). A Mohr-Coulomb type curve is then fit to the results before being linearized to compute the intrinsic parameters. The mass range to obtain different loads goes from $0.176 \mathrm{~g}$ to $0.546 \mathrm{~g}$. The curve is a power equation of the form:

$$
\tau=a \sigma_{n}^{b}+c
$$

Where $\tau$ is the shear stress, $\sigma_{n}$ is the normal stress, and $a, b, c$ are constants defining the curve. The normal stress is obtained from the range of weights available, using Eq.5.11.

\subsubsection{Results of tests in laboratory setting 6.3.1.2.1 Clay of low plasticity (USCS CL)}

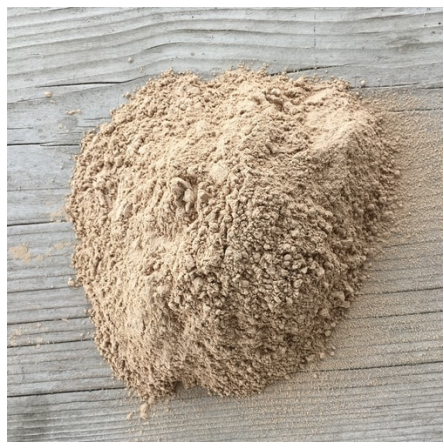

(a) Dry clay of low plasticity. Expected $c=86 \mathrm{kPa}$ and $27^{\circ} \leq \phi \leq$ $35^{\circ}$.

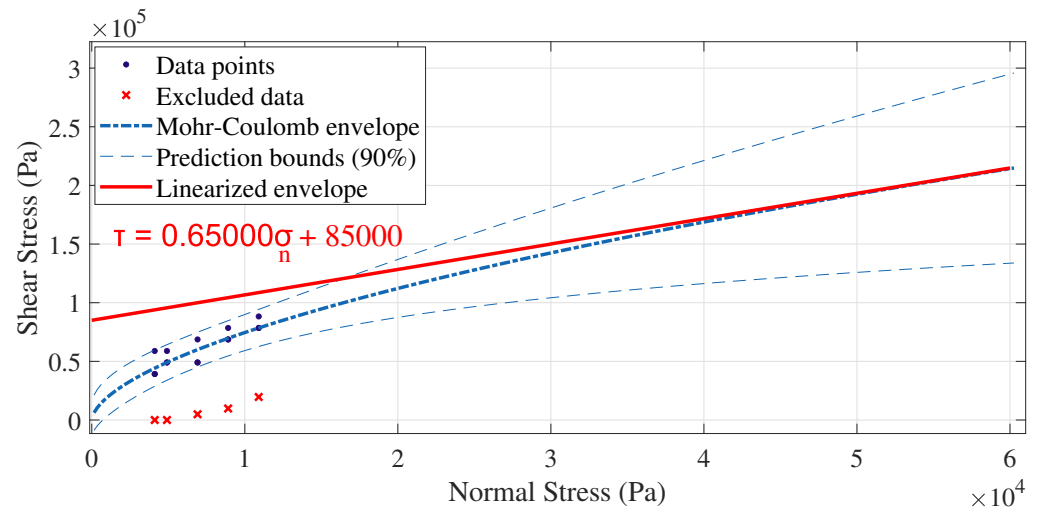

(b) Mohr-Coulomb envelope and linearization giving $c=85 \mathrm{kPa}$ and $\phi=33^{\circ}$.

Figure 6.14: Clay of low plasticity. Results give $c=85 \mathrm{kPa}$ and $\phi=33^{\circ}$. Some data points overlap with each other. Photo credit: agrclassroomstore.com.

Dry clay is of low plasticity, with an expected cohesion of $86 \mathrm{kPa}$ when compacted, and an angle of internal friction between $27^{\circ}$ and $35^{\circ}$. The tests results for compacted dry clay are presented in Fig.6.14. One set of tests has been eliminated due to inadequate testing, showing results significantly lower than the rest of the points.

The tests gave a cohesion $c=85 \mathrm{kPa}$ and an angle of internal friction $\phi=\operatorname{atan}(0.6500)=$ $33.02^{\circ}$. The Mohr-Coulomb curve is given by Eq.6.1 with the following coefficient (with $95 \%$ confidence bound) $a=327.8(-312.6,968.2), b=0.5892(0.3713,0.8072)$ and $c=0$. The goodness of fit for dry clay is characterize by $R^{2}=0.7469$. 


\subsection{Silt loam, compacted (USCS ML, OL, MH, OH)}

Next, compacted silt loam is tested. The sample is placed in a container and manually compacted by applying a load to the sample before the tests were conducted. Compacted silt loam has a cohesion of 60 to $90 \mathrm{kPa}$ and an angle of internal friction between $25^{\circ}$ and $32^{\circ}$. The results are presented in Fig.6.15. The compacted silt loam led to the following results: $c=90 \mathrm{kPa}$ and

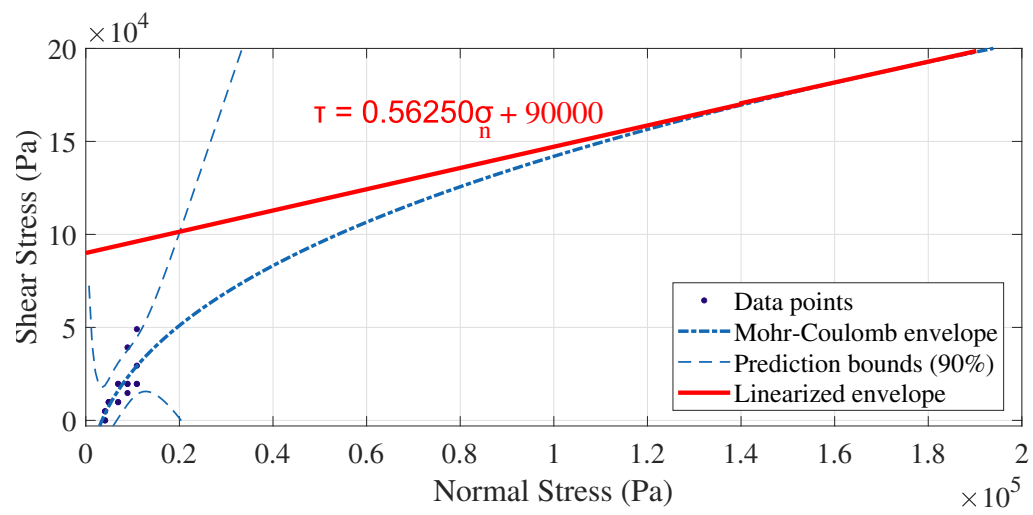

(a) Mohr-Coulomb envelope and linear approximation giving $c=90 \mathrm{kPa}$ and $\phi=29^{\circ}$.

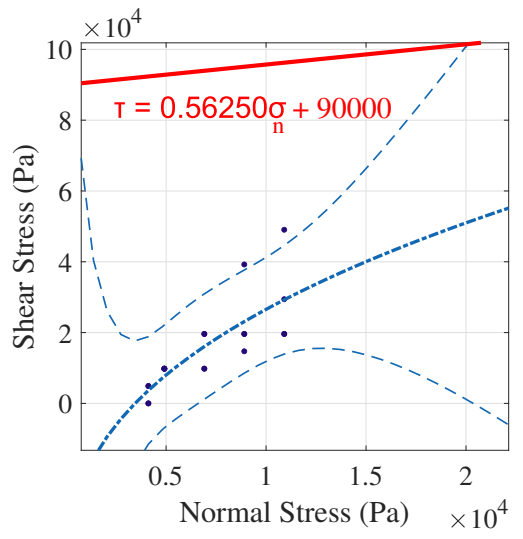

(b) Zoom on the part of the curve with data points.

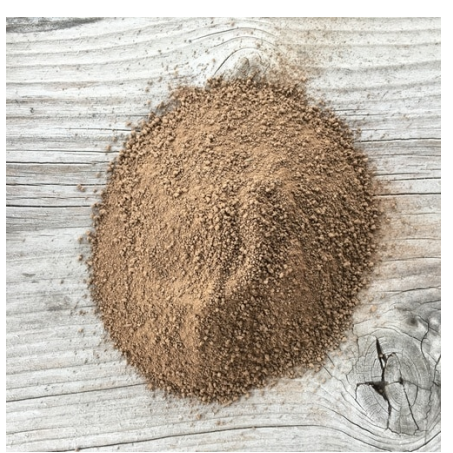

(c) Silt Loam. Expected $c=60-90 k P a$ and $25^{\circ} \leq \phi \leq$ $32^{\circ}$.

Figure 6.15: Compacted silt loam. Results give $c=90 k P a$ and $\phi=29^{\circ}$. Some data points overlap with each other. Photo credit: agrclassroomstore.com.

$\phi=\operatorname{atan}(0.5625)=29.36^{\circ}$. The Mohr-Coulomb curve is given by Eq.6.1 with the following coefficient (with 95\% confidence bound) $a=1960(-6.63 e 04,7.022 e 04), b=0.3983(-2.671,3.468)$ and $c=-5.027 e 04(-5.559 e 05,4.554 e 05)$. The goodness of fit for compacted silt loam is characterize by $R^{2}=0.6234$.

\subsection{Fine sand (USCS SW, SP)}

The third sample material tested is well graded, fine grain, cohesionless sand with an expected angle of internal friction ranging between $36^{\circ}$ and $41^{\circ}$.

The results show $c=-0.9278 k P a$ and $\phi=\operatorname{atan}(0.4639)=24.88^{\circ}$ for fine, well graded sand. The Mohr-Coulomb curve is given by Eq.6.1 with the following coefficient (with 


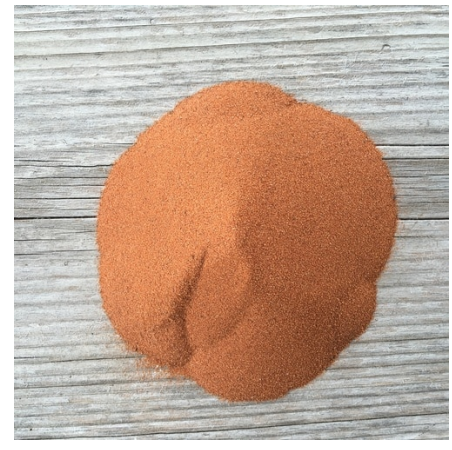

(a) Fine grain, well graded sand. Expected $c=0 k P a$ and $36^{\circ} \leq \phi \leq 41^{\circ}$

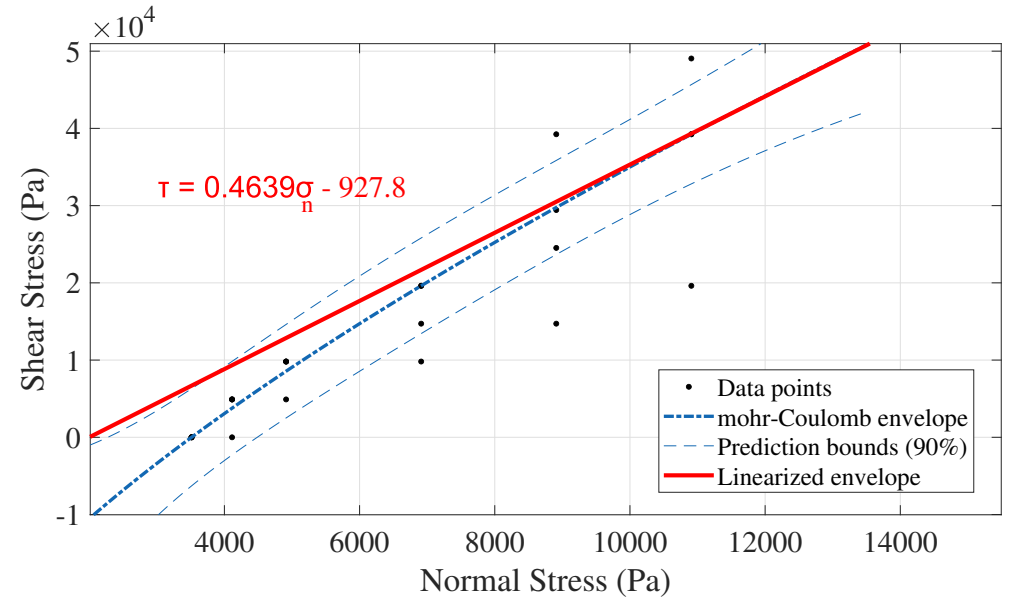

(b) Mohr-Coulomb envelope and linear approximation giving $c=-1 \mathrm{kPa}$ and $\phi=25^{\circ}$

Figure 6.16: Tests in fine grain sand material of known parameters. Photo credit: agrclassroomstore.com.

95\% confidence bound) $a=102.7(-639.8,845.2), b=0.7039(-0.003083,1.411)$ and $c=$ $-3.217 e 04(-8.106 e 04,1.671 e 04)$. The goodness of fit for fine, well graded sand is characterize by $R^{2}=0.9463$.

\subsubsection{Results of tests in field setting \\ 6.3.1.3.1 Sand (USCS SW, SP)}

A sand pit under dry conditions is chosen, where the cohesion is expected to be $0 k P a$ (cohesionless) and the angle of internal friction is usually between $37^{\circ}$ and $38^{\circ}$ for such material. The results are presented in Fig.6.17b, where the resulting Mohr-Coulomb envelope is shown.

From the plotted data in Fig. 6.17, the retrieved cohesion is $c=10.5 \mathrm{kPa}$ and the angle of internal friction is $\phi=\operatorname{atan}(0.0800)=4.57^{\circ}$. The Mohr-Coulomb curve is given by 6.1 with the following coefficient (with 95\% confidence bound) $a=-2.615 e 11,(-5.793 e 12,5.27 e 12$ ), $b=-2.095(-4.723,0.5321)$ and $c=1.217 e 04(7598,1.675 e 04)$. The goodness of fit of the MohrCoulomb envelope is characterized by $R^{2}=0.9652$

\subsection{Silt loam, saturated (USCS ML, OL, MH, OH)}

Another experiment is conducted in the field, in saturated silt loam. The results are presented in Fig. 6.18.

The retrieved cohesion from Fig. 6.18 is $c=20 k P a$ and friction angle is $\phi=\operatorname{atan}(0.5333)=$ $28.07^{\circ}$. The Mohr-Coulomb curve is given by Eq.6.1 with the following coefficient (with $95 \%$ confidence bound) $a=-2.544 e 10(-2.006 e 12,1.955 e 12), b=-1.885(-11.6,7.829)$ and $c=$ $2.108 e 04(1.007 e 04,3.209 e+04)$. The goodness of fit of the Mohr-Coulomb envelope for this test is characterized by $R^{2}=0.6821$.

\subsubsection{Interpretation of results}

The results are summarized in Table 6.2b. Overall, the tests give cohesions and angles of internal friction within the expected range for the material. The exceptions to the rule are the sand 


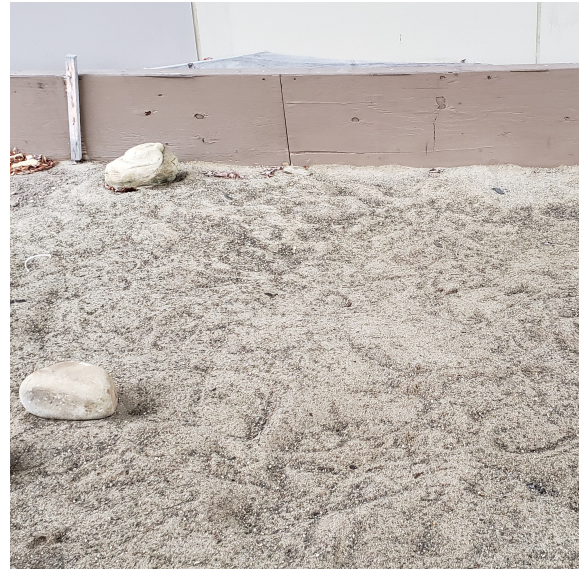

(a) Sand pit in which tests were performed. $c=0$ and $\phi=37^{\circ}-38^{\circ}$.

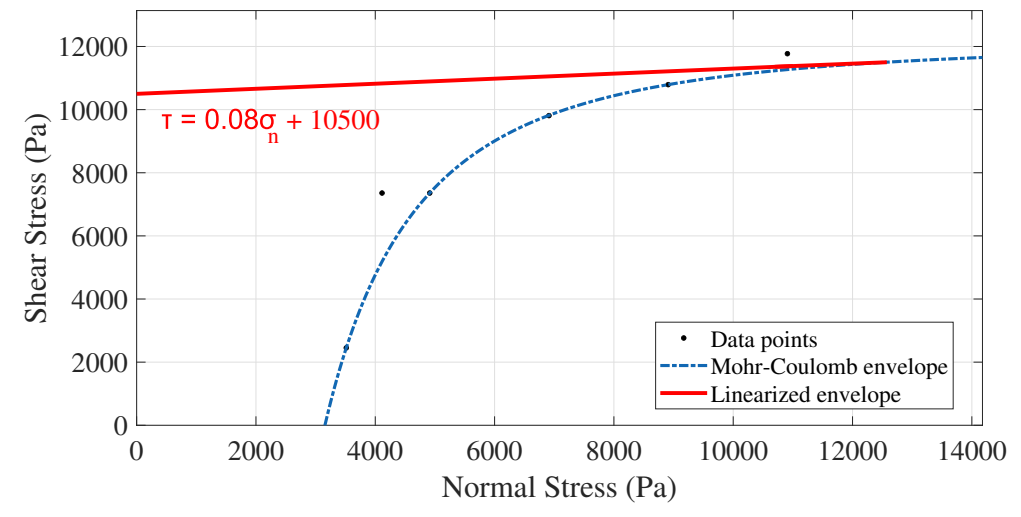

(b) Mohr-Coulomb envelope and linear approximation giving $\mathrm{c}=10.5 \mathrm{kPa}$ and $\phi=$ $4.6^{\circ}$

Figure 6.17: Test of the pocket shear vane in cohesionless sand under dry conditions. Photo credit: G. Hedrick.

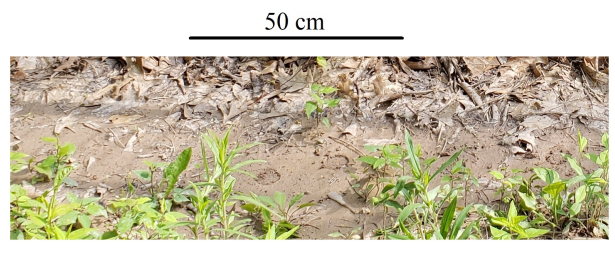

(a) Cheat Lake site, expected $c=10 k P a-20 k P a$ and $\phi=25^{\circ}-32^{\circ}$.

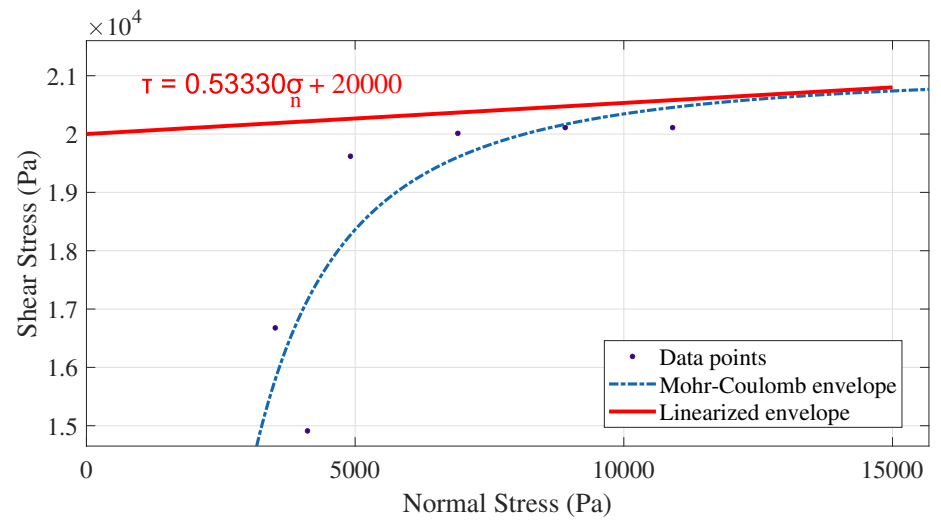

(b) Mohr-Coulomb envelopes and linear approximation giving $c=20 \mathrm{kPa}$ and $\phi=$ $28^{\circ}$.

Figure 6.18: Hand-held instrument tested in Silt Loam, saturated (in-situ). Photo credit: G. Hedrick.

samples, that give a negative cohesion in one case and an unrealistic angle of internal friction in the other. This shows the limitation of the proposed method to cohesive soils only, known to be present on Mars [Sullivan et al., 2011].

\subsubsection{Mounted shear vane}

\subsubsection{Testing settings}

Given the results of the hand-held pocket shear vane, that it generally does not operate correctly in cohesionless soil, the controlled sample retained for testing the mounted instrument is the clay of low plasticity (USCS CL). Several tests are run for each load (five per load), and 
Table 6.2: Results of cohesion and angle of internal friction from testing. In green are adequate results, and in red, inadequate results.

(a) Results of cohesion measurements compared against expected values.

\begin{tabular}{||c|c||c|c||}
\hline Description & USCS & Expected (kPa) & Result (kPa) \\
\hline \hline \multicolumn{3}{|c||}{ Controlled samples } \\
\hline Clay of low plasticity, compacted & CL & 86 & 85 \\
\hline Silt loam, compacted & ML, OL, MH, OH & $60-90$ & 90 \\
\hline Sand, fine grain, well graded & SW, SP & 0 & -1 \\
\hline \hline \multicolumn{4}{|c|}{ Field testing } \\
\hline Sand & SP & 0 & 10.5 \\
\hline Silt loam, saturated & ML, OL, MH, OH & $10-20$ & 20 \\
\hline
\end{tabular}

(b) Results of angle of internal friction measurements compared against expected values.

\begin{tabular}{||c|c||c|c||}
\hline Description & USCS & Expected $\left(^{\circ}\right)$ & Result $\left(^{\circ}\right)$ \\
\hline \hline \multicolumn{3}{|c||}{ Controlled samples } \\
\hline Clay of low plasticity, compacted & CL & $27-35$ & 33 \\
\hline Silt loam, compacted & ML, OL, MH, OH & $25-32$ & 29 \\
\hline Sand, fine grain, well graded & SW, SP & $36-41$ & 25 \\
\hline \hline \multicolumn{4}{|c||}{ Field testing } \\
\hline Sand & SW, SP & $37-38$ & 4.9 \\
\hline Silt loam, saturated & ML, OL, MH, OH & $25-32$ & 28 \\
\hline
\end{tabular}

a Mohr-Coulomb type curve (see 6.1) is fit to the results before being linearized to compute the intrinsic parameters. The loads are obtained for masses ranging from $100 \mathrm{~g}$ to $500 \mathrm{~g}$.

The instrument is then tested in the saturated silt loam (USCS ML, OL, MH, OH) with only three sets of data per load, to stay as close as possible to the conditions in which it would operate on Mars. The normal stress is obtained from the range of weights available, using Eq.5.11. Due to the unpredictability of field testing, results that are known to be unrealistic are omitted from the data. For example, when the instrument is not properly deployed (such as tilted, not touching the ground evenly, etc...) the resulting values are excluded. Moreover, the potentiometer consistently output impossible results and therefore, it was quickly discarded from the analysis. All results presented in this section are thus derived from the load cell outputs.

\subsubsection{Results of tests in laboratory setting}

The retrieved cohesion is $c=90 k P a$ and angle of internal friction is $\phi=\operatorname{atan}(0.6667)=$ $33.69^{\circ}$. Unlike the tests with the hand-held instruments, the data for the mounted shear vane are more widespread, leading to a goodness of fit characterized by $R^{2}=0.3466$. The variables' values for Eq.6.1 are the following (with a 95\% confidence bound): $a=2.338 e+05(-1.601 e+$ $07,1.648 e+07), b=0.06755(-2.917,3.052)$ and $c=-3.632 e+05(-1.866 e+07,1.794 e+$ 07). The estimated maximum power consumption is $83.056 \mathrm{~W}$, and the total power consumption of the payload per data collection should not exceed $2.5 k J$. These values do not include the 


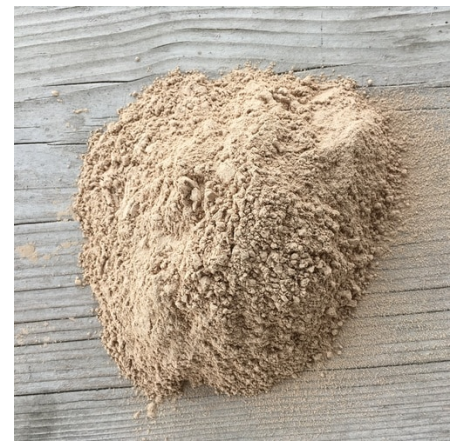

(a) Dry clay of low plasticity. Expected $c=86 \mathrm{kPa}$ and $27^{\circ} \leq \phi \leq$ $35^{\circ}$.

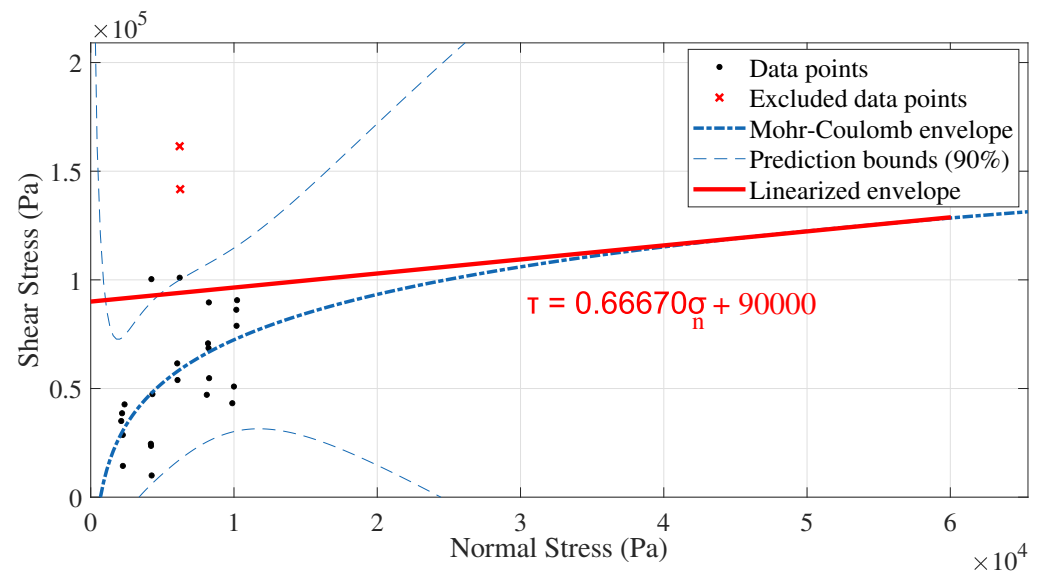

(b) Mohr-Coulomb envelope and linearization giving $c=90 \mathrm{kPa}$ and $\phi=34^{\circ}$.

Figure 6.19: Mounted instrument tested in Clay of low plasticity, compacted. Results give $c=90 k P a$ and $\phi=$ $34^{\circ}$. Some data points overlap with each other, while others were eliminated due to being unrealistics. Photo credit: agrclassroomstore.com.

resources expended to transition the rover from a driving stance to a squat in order to conduct the experiment, or the minute movement needed to position the mounted shear vane over fresh soil for a new measurement.

\subsubsection{Results of tests in field setting}

The retrieved cohesion is $c=20 k P a$ and angle of internal friction is $\phi=\operatorname{atan}(0.4333)=$ $23.43^{\circ}$. Unlike the tests with the hand-held instruments, the data for the mounted shear vane are more widespread, and a lot of points had to be eliminated because the position of the instrument led to false readings. The goodness of fit for the curve is characterized by $R^{2}=0.8938$. The variables' values for Eq.6.1 are the following (with a 95\% confidence bound): $a=2.338 e+05(-1.601 e+$ $07,1.648 e+07), b=0.06755(-2.917,3.052)$ and $c=-3.632 e+05(-1.866 e+07,1.794 e+07)$.

\subsubsection{Interpretation of results}

The mounted shear vane appears to be sensitive to its tilt and gives false results when operating at an angle. Therefore, many data points are ignored because of inadequate leveling of the payload. The overall results are presented in Table 6.3. The values are not as accurate as they are with the hand-held instrument, due to the mounted shear vane being sensitive to testing conditions. However, it remains within 5\% of the expected value, which is acceptable (e.g., cohesion of $90 k P a$ instead of $86 \mathrm{kPa}$ ).

\subsubsection{Estimate of ultimate bearing capacity for various soils}

In section 5.4.2.1 it was shown that an estimate of the ultimate bearing capacity $q_{u l t}$ can be derived from plotting the Mohr circle at the point of failure. The results for each tested soil are presented below. 


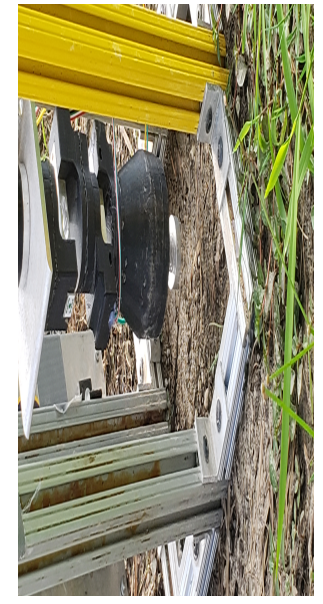

(a) Silt loam, saturated. Expected $c=10-20 k P a$ and $25^{\circ} \leq \phi \leq$ $32^{\circ}$.

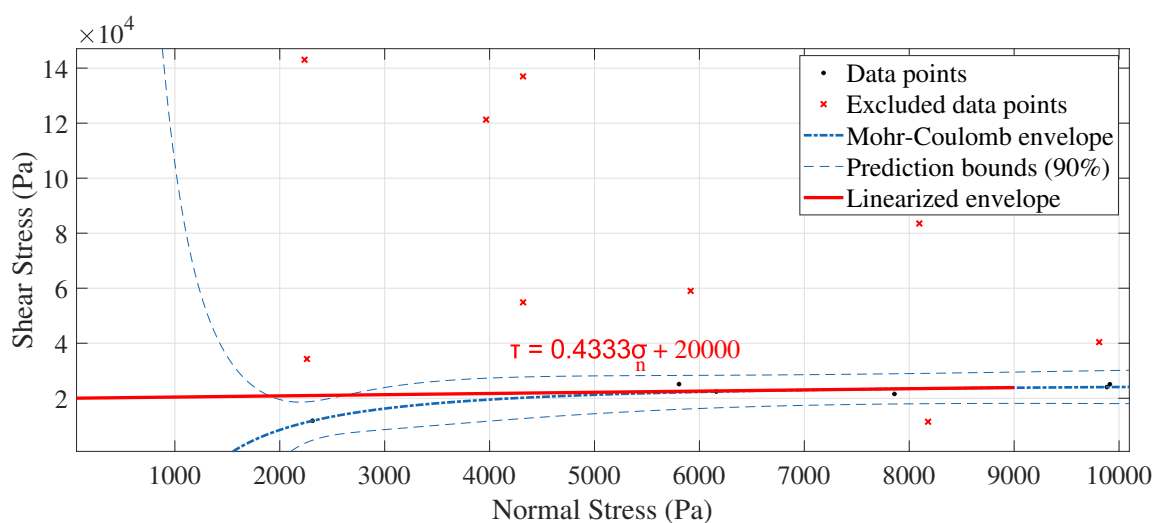

(b) Mohr-Coulomb envelope and linearization giving $c=20 \mathrm{kPa}$ and $\phi=23.5^{\circ}$.

Figure 6.20: Mounted instrument tested in Silt loam, saturated (in-situ). Results give $c=20 k P a$ and $\phi=24^{\circ}$. Some data points overlap with each other, while others were eliminated. Photo credit: G. Hedrick.

Table 6.3: Results of cohesion and angle of internal friction from testing.

(a) Results of cohesion measurements compared against expected values.

\begin{tabular}{||c|c||c|c||}
\hline Description & USCS & Expected (kPa) & Result (kPa) \\
\hline \hline \multicolumn{3}{|c||}{ Controlled samples } \\
\hline Clay of low plasticity, compacted & CL & 86 & 90 \\
\hline \hline \multicolumn{4}{|c|}{ Field testing } \\
\hline Silt loam, saturated & ML, OL, MH, OH & $10-20$ & 20 \\
\hline
\end{tabular}

(b) Results of angle of internal friction measurements compared against expected values.

\begin{tabular}{||c|c||c|c||}
\hline Description & USCS & Expected $\left({ }^{\circ}\right)$ & Result $\left(^{\circ}\right)$ \\
\hline \hline \multicolumn{4}{|c||}{ Controlled samples } \\
\hline Clay of low plasticity, compacted & CL & $27-35$ & 34 \\
\hline \hline \multicolumn{4}{|c||}{ Field testing } \\
\hline Silt loam, saturated & ML, OL, MH, OH & $25-32$ & 23.5 \\
\hline
\end{tabular}

\subsubsection{Hand-held shear vane: derived Mohr circles}

For both clay and silt loam, the Mohr circles at the point of failure are plotted and the results are shown in Fig.6.21. The same analysis can be performed with in-situ data as shown in Fig.6.22. The compacted clay and silt loam show $q_{u l t}$ of $310 \mathrm{kPa}$ and $308 \mathrm{kPa}$, whereas in-situ saturated silt loam can only bear about $66 \mathrm{kPa}$ maximum. 


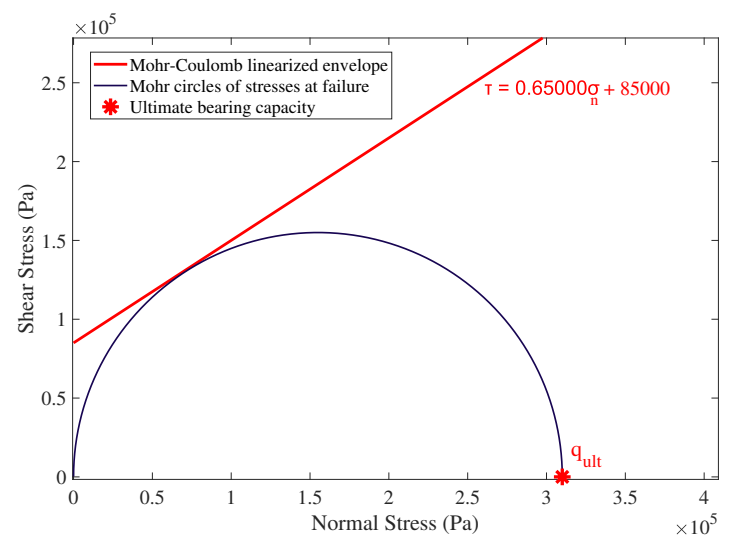

(a) Mohr circle at failure and Mohr-Coulomb envelope for clay of low plasticity, compacted.

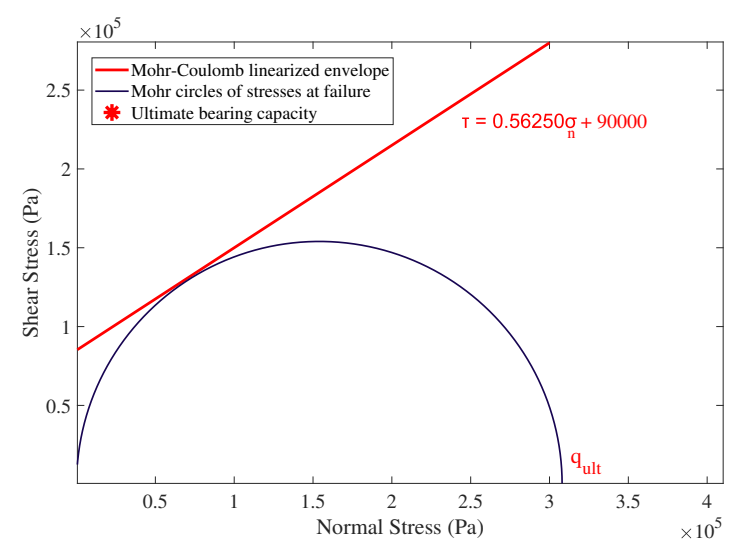

(b) Mohr circle at failure and Mohr-Coulomb envelope for silt loam, compacted.

Figure 6.21: Estimation of the ultimate bearing capacity for laboratory tests. Compacted clay shows $q_{u l t}=310 \mathrm{kPa}$ and compacted silt loam can hold up to $q_{u l t}=308 \mathrm{kPa}$.

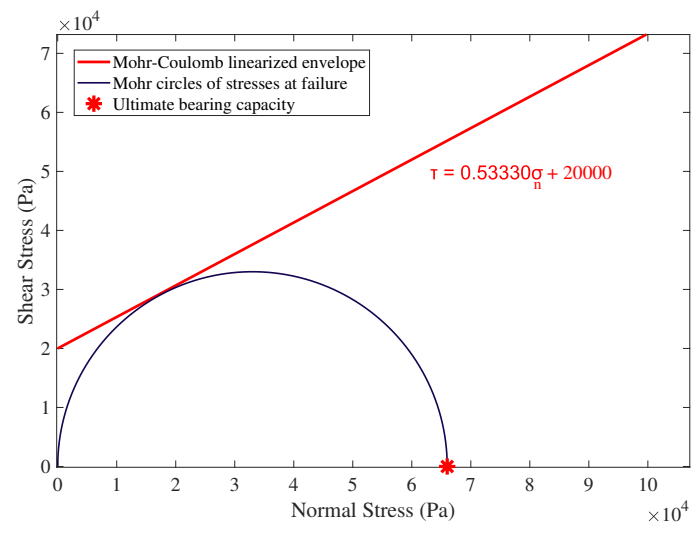

Figure 6.22: Mohr circle at failure and Mohr-Coulomb envelope for in-situ silt loam, saturated. The result show that saturated silt loam can bear $q_{u l t}=66 \mathrm{kPa}$ at most.

\subsubsection{Mounted shear vane: derived Mohr circles}

The same analysis than previously presented for the hand-held device is performed for the mounted shear vane, with the plots shown in Fig.6.23. The same soils analyzed with curves obtained from the mounted instruments give similar results to what was derived from the Mohr circles of the hand-held shear vane: the compacted clay shows $q_{u l t}=330 \mathrm{kPa}$ and the saturated silt loam tested in-situ can hold up to $60 \mathrm{kPa}$.

\subsubsection{Overall results}

The results are summarized in Table 6.4 and compared with values widely used in civil engineering for the study of foundations [NYC, 2020]. While the allowable bearing capacity $q_{a}$ is the value used for construction, the ultimate bearing capacity is easily found by converting $q_{a}$ to 


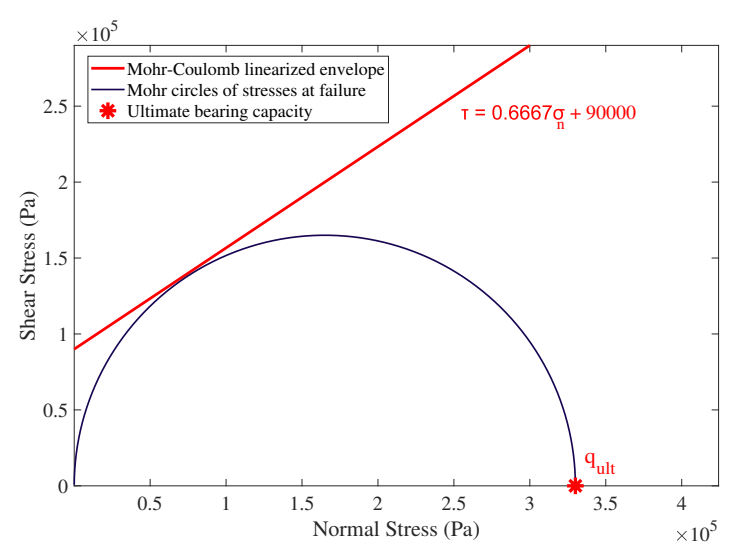

(a) Mohr circle at failure and Mohr-Coulomb envelope for clay of low plasticity, compacted.

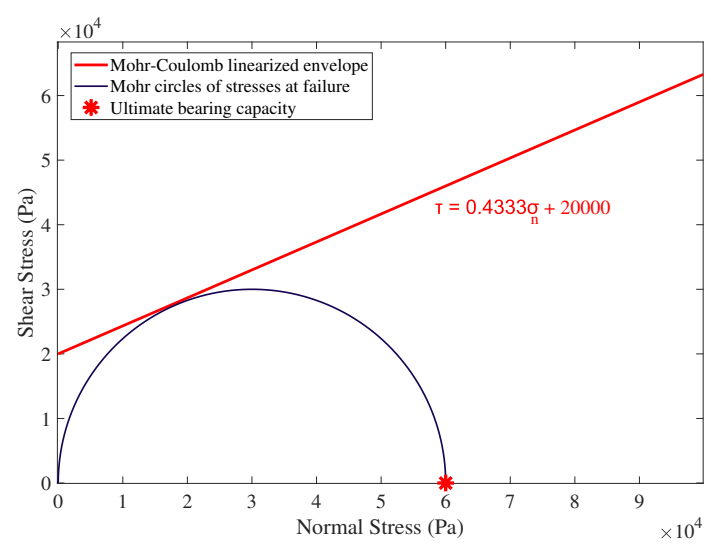

(b) Mohr circle at failure and Mohr-Coulomb envelope for in-situ silt loam, saturated.

Figure 6.23: Estimation of the ultimate bearing capacity for laboratory and field tests. Compacted clay shows $q_{u l t}=$ $330 \mathrm{kPa}$ and saturated silt loam can hold up to $q_{u l t}=60 \mathrm{kPa}$.

$q_{u l t}$ according to the following formula [Das, 2007]:

$$
q_{a}=\frac{q_{u l t}}{F_{s}}
$$

with $F_{s}$ the factor of safety, usually ranging from 2 to 5 , although typically set to 3 [Terzaghi and Peck, 1948]. The values for the ultimate bearing capacity derived for all tested soils fall within the expected range for such terrains, indicating that the method can be used for rover missions to obtain an estimate of $q_{u l t}$ on Martian terrains.

Table 6.4: Estimated ultimate bearing capacity for various soils derived from the Mohr circles at the point of failure.

\begin{tabular}{||l|l||c|c|c||}
\hline Description & USCS & Maximum $q_{a}$ & Minimum $q_{u l t}\left(F_{s}=2\right)$ & Result (kPa) \\
\hline \hline \multicolumn{5}{|c||}{ Controlled samples } \\
\hline $\begin{array}{l}\text { Clay of low plasticity, } \\
\text { compacted }\end{array}$ & $\mathrm{CL}$ & $192-479$ & 384 & $310-330$ \\
\hline Silt loam, compacted & $\begin{array}{l}\text { ML, OL, } \\
\text { MH, OH }\end{array}$ & $144-287$ & 288 & 308 \\
\hline \hline \multicolumn{5}{|c||}{ Field testing } \\
\hline Silt loam, saturated & $\begin{array}{l}\text { ML, OL, } \\
\text { MH, OH }\end{array}$ & $<37.5$ & $<75$ & $60-66$ \\
\hline
\end{tabular}

\subsubsection{Conclusion}

As seen with the hand-held experiments, the modified pocket shear vane leads to useful results of cohesion and internal friction that can be used to compute parameters of cohesive soils. From such results, an estimate of the ultimate bearing capacity of each soil is also successfully 
derived. When mounted, the instrument performs adequately as well, and is capable of giving intrinsic parameters needed to identify a terrain, as well as an estimate of its ultimate bearing capacity that can indicate when soil failure will occur under the rover's wheels. The pocket shear vane's ease of use, its light weight, compact form and versatile function makes it a great candidate for planetary surface missions.

The transition to remote operation shows that many improvements can be made to the mounted instrument. One way to improve its operation involves selecting components that are better suited for such a design. For instance, the current linear servo struggles to precisely apply axial load, especially at weights higher than $3 \mathrm{~kg}$. A lead screw and stepper motor would be able to apply force more precisely than the potentiometer-based linear servo. Also due to lack of linear servo precision in the current prototype, it was found that the device performs better under light loads (for masses between 100 - $500 \mathrm{~g}$ ). In the future, utilizing more sensitive load cells that have a maximum weight closer to the light loads targetted in the experiment will greatly increase the accuracy of individual measurements. It should also be noted that the prototype shown in this dissertation is a proof of concept, and the device performance will be improved in the next version by utilizing higher quality components that will increase stiffness and precision of the overall mechanism.

In addition to improving on the current design to obtain more accurate results, future work includes testing under a wider range of conditions, as well as developing an algorithm to automatically interpret the results and compute the cohesion and angle of internal friction on board. Additionally, this instrument will be mounted on a physical robot currently under construction at WVUIRL, and further testing will be conducted with the shear vane fully integrated. This research will also lead to studying other types of instruments, such as a cone penetrometer, to be able to predict soil parameters in cohesionless terrain.

\subsection{Summary of results}

This Chapter demonstrates that the proposed approach to increase autonomy by useful results. From orbit, traversability information such as expected velocity and slip range can be retrieved. From the surface, a fast and efficient terrain monitoring via terramechanics supports planning and map update effectively. Finally, a shear vane to further assist the rover if needed shows that the traversability can be analyzed at all times, even when monitoring does not provide conclusive results. 


\section{CHAPTER 7 Discussion and Conclusion}

This research implements a novel approach to planetary rover traverse planning by fully integrating terrain information into the planning phase. At different levels, it supports increased autonomy of future Mars rovers, specifically for applications to a sample return mission. This work proposes to gather various terrain information from orbit to support planning and further assist the rover from the surface with a fast and efficient terrain monitoring method. This research also presents a complete process to reduce uncertainty in identifying a terrain, should the main method be insufficient to give the rover proper knowledge of its surrounding. This framework aligns well with requirements for a sample return mission, as it provides a method to fast traverse partially unknown terrains.

\subsection{Discussion and future work}

\subsubsection{Map of traversability information}

Orbital data can be processed to derive a map containing traversability information, more specifically expected rover speed and wheel slip. The combination of data sets has been shown to effectively predict rover velocity, and the classifier trained with thermal inertia and topography has demonstrated its ability to give a range of expected slip with an acceptable accuracy relying only on orbital imagery. This information can then be used to plan a traverse more effectively, without the need for ground data, and limit the potential encounters of mobility difficulties (for example, areas showing more than $30 \%$ slip could be avoided). Such a map can help enhance rover autonomy, needed for a sample return mission.

While the expected velocity map can be derived at a scale as up as $1 \mathrm{~m} / \mathrm{px}$, the prediction of slip is a lot more affected by data resolution. Future work includes studying the possibility of interpolating thermal inertia to higher resolution using other data sets such as HiRISE or CRISM (Compact Reconnaissance Imaging Spectrometer for Mars), the latter allowing for derivation of spectra over selected areas at a resolution between $12 \mathrm{~m} / \mathrm{px}$ and $18 \mathrm{~m} / \mathrm{px}$ [Fox et al., 2016]. This interpolation can be supported by ground data provided by the mini-TES instrument (mini-Thermal Emission Spectrometer) on board the twin rovers Spirit and Opportunity [Fergason et al., 2006a].

\subsubsection{Terrain-aware path planning}

The local path planning method successfully integrates terrain information, at the rover location and ahead of the vehicle, and implements a fast replanning process by having many path options before starting the drive each sol. Terrain information is obtained via monitoring of rover slip and actual drive speed, then propagated along the different paths that are at the rover's disposal at all times. This information is compared against the prior knowledge of the rover and if there are 
discrepancies, the map of velocity is updated locally. The terrain monitoring affects the local area and effectively supports planning by allowing for fast and efficient replanning.

If the algorithm captures correctly the actual terrain in the vicinity of the rover, it should be noted that it relies on new ground information given prior to driving. The initialization of the map reflects potential undetected terrain ahead that can be confirmed or refuted by the belief propagation framework. For simulation purposes, high resolution orbital images were used for the initialization (e.g., HiRISE), but for MSR, the ground data from Perseverance can be utilized instead. Future work regarding the path planning method includes: further development of the algorithm to overcome the need for a secondary assessment using ground images; research on integration of lower-level motion planning algorithms and online risk assessment; and decision making under unexpected situations. These results will also be tested on a physical robot in a Mars analog environment, at which point the "truth" map would be replaced by actual monitoring of the terrain.

\subsubsection{Instrument deployment in support of planning}

The instrument chosen to give in-situ information with reduced uncertainty about the terrain to be fed into the planning process in support of noisy measurements successfully fulfills its requirements by outputting valid values of intrinsic parameters and bearing capacities for a given soil. Moreover, the instrument can be deployed easily and give results in a few minutes, which is in line with the objectives of a sample return mission.

This prototype is in the early stages of development. Many potential improvements have already been identified in section 6.3.4 that target the material (e.g., higher quality components) or choice of parts (e.g., more sensitive load cells). More work will also be done to implement an automatic result interpretation procedure. The next step is to improve the design to better adapt to field testing, mainly, to overcome the sensitivity to tilt that could become a liability when deployed in a Mars-like environment. Moreover, this instrument will eventually be mounted on the rover developed at WVUIRL (therefore not relying on linear guide rails for use) and tested in Marslike conditions. This involves integrating the deployment of the shear vane to the terrain-aware planning algorithm presented in section 5.3, where it would be used when an exact measurement is triggered.

\subsubsection{Terrain-aware traverse planning as a framework to support a sample return mis- sion}

While this work has been presented as three separate sections, the proposed framework is an integration of all three parts into overall terrain-aware traverse planning, with an orbital analysis of the area used to plan the path and terrain assessment methods to correct any discrepancies found in the aforementioned analysis. This new approach to planning a rover mission on the surface of Mars shows promising results, as shown in Chapter 6. Future research has been identified throughout this work that would strengthen the proposed approach, which could eventually lead to a novel process to tackle tactical operations for MSR. 


\subsection{Conclusion}

At different levels, this research offers a framework that complies with the requirements of a Mars Sample Return mission, such as faster traverse and increased autonomy. The proposed approach analyzes the landing area from orbit to surface in a series of steps aimed at providing a safer and more autonomous way to plan and complete a traverse: first, the site is studied from orbit to gather traversability information about the terrain. This translates into a map of expected wheel slip and rover speed that can fully support planning. Next, this research suggests an effective method to plan the traverse for the driving objective of a sol using the aforementioned map. An easy replanning process that saves computational resources is implemented by computing offline several options, prior to driving, that the rover can choose from in real-time, should it be required to deviate from its initial route. To promote rover safety and ensure the adequacy of the information about the environment given by the map, a simple and fast method to gather terrain information is proposed, with the rover analyzing its wheel slip and actual speed and converting them into useful data that can confirm the a priori information. This research demonstrates that the rover can successfully update its map of expected velocity on a local scale if it discovers discrepancies with a priori values. This work also implements a feature that complements terrain monitoring with an easy to use, compact, light-weigh instrument that gives terrain information with certainty in a timely manner, therefore supporting effective planning.

This is summarized in Fig.7.1 and shows that all parts of this work are strongly connected to each other. The path planning relies on the analysis of orbital data, while its execution depends on terrain assessment through monitoring and in-situ analysis. Figure 7.1 shows that the map, constructed using raw and processed orbital data, is the foundation for planning the drive for a sol. During the traverse, the rover gathers terrain information to confirm traversability, and when it does not match the aforementioned map, an update is performed around the rover location. This locally updated version is then used in planning the following sol. The loop continues until the rover reaches the samples and completes its mission. As it retains information about prior changes, it acquires new and more adequate knowledge about its environment and renders the following planning phases less challenging. Thus, the overall framework proposed in this research supports a more autonomous rover, suitable for Mars Sample Return. 


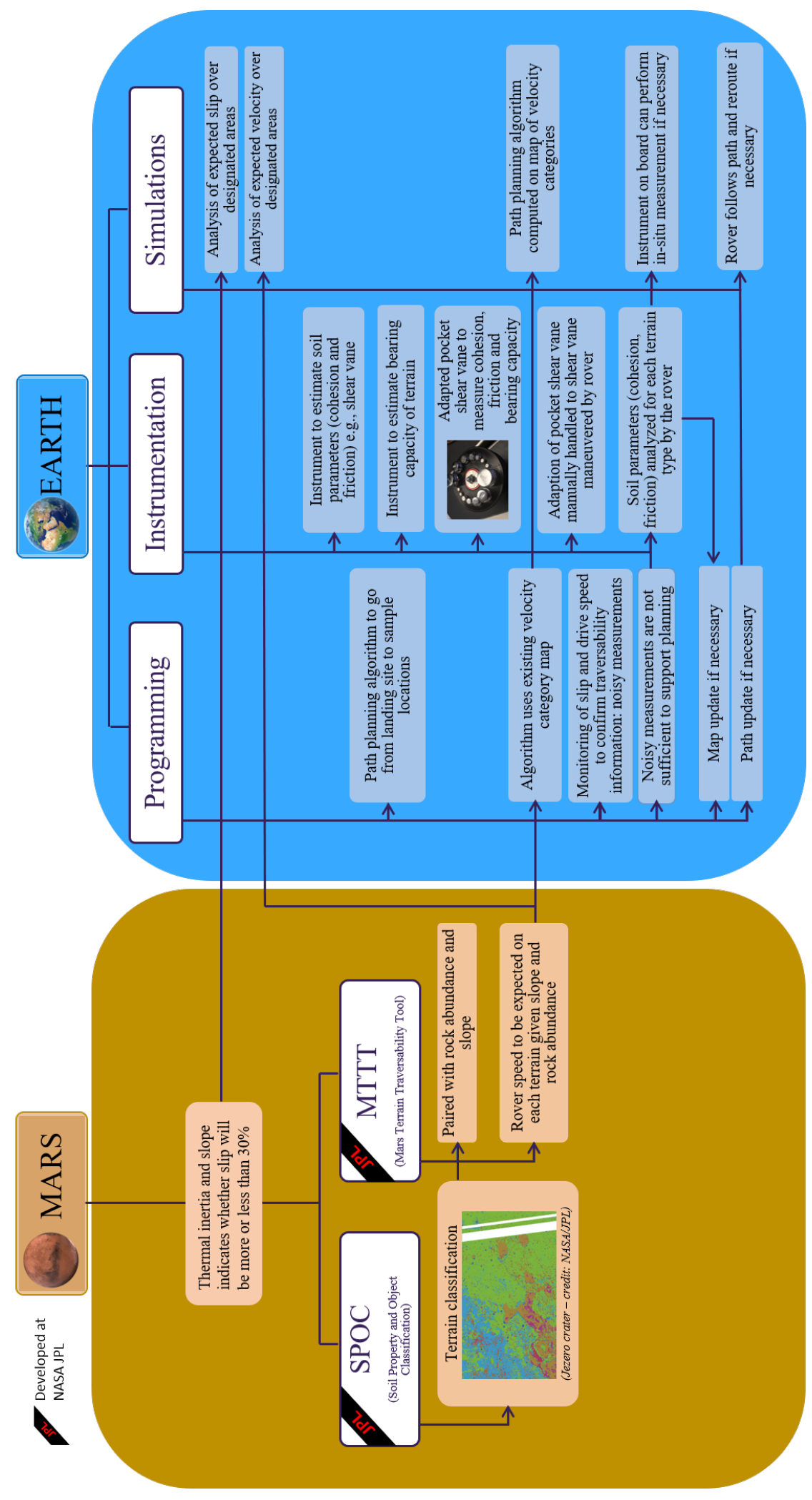

Figure 7.1: Framework supporting more autonomy for a Mars Sample Return mission. 


\section{Bibliography}

[Aberdeen, 2003] Aberdeen, D. (2003). A (revised) survey of approximate methods for solving partially observable markov decision processes. Technical report, Technical report, National ICT Australia.

[Agarwal et al., 2019] Agarwal, S., Senatore, C., Zhang, T., Kingsbury, M., Iagnemma, K., Goldman, D. I., and Kamrin, K. (2019). Modeling of the interaction of rigid wheels with dry granular media. Journal of Terramechanics, 85:1-14.

[Agha-Mohammadi et al., 2014] Agha-Mohammadi, A.-A., Chakravorty, S., and Amato, N. M. (2014). Firm: Sampling-based feedback motion-planning under motion uncertainty and imperfect measurements. The International Journal of Robotics Research, 33(2):268-304.

[Amar and Bidaud, 1997] Amar, F. B. and Bidaud, P. (1997). Dynamic analysis of off-road vehicles. In Experimental Robotics IV, pages 363-371. Springer.

[Arora et al., 2016] Arora, A., Fitch, R., and Sukkarieh, S. (2016). Extending scientific information gathering by modeling geological knowledge in a bayesian framework.

[Arvidson et al., 2014] Arvidson, R., Bellutta, P., Calef, F., Fraeman, A., Garvin, J. B., Gasnault, O., Grant, J. A., Grotzinger, J., Hamilton, V., Heverly, M., et al. (2014). Terrain physical properties derived from orbital data and the first 360 sols of mars science laboratory curiosity rover observations in gale crater. Journal of Geophysical Research: Planets, 119(6):1322-1344.

[Arvidson et al., 2017] Arvidson, R., DeGrosse Jr, P., Grotzinger, J., Heverly, M., Shechet, J., Moreland, S., Newby, M., Stein, N., Steffy, A., Zhou, F., et al. (2017). Relating geologic units and mobility system kinematics contributing to curiosity wheel damage at gale crater, mars. Journal of Terramechanics, 73:73-93.

[Arvidson, 2014] Arvidson, R. E. (2014). Roving on mars with opportunity and curiosity: terramechanics and terrain properties. In Earth and Space 2014, pages 165-173.

[Arvidson et al., 2011] Arvidson, R. E., Ashley, J. W., Bell, J., Chojnacki, M., Cohen, J., Economou, T., Farrand, W. H., Fergason, R., Fleischer, I., Geissler, P., et al. (2011). Opportunity mars rover mission: Overview and selected results from purgatory ripple to traverses to endeavour crater. Journal of Geophysical Research: Planets, 116(E7).

[Bajracharya et al., 2008] Bajracharya, M., Maimone, M. W., and Helmick, D. (2008). Autonomy for mars rovers: Past, present, and future. Computer, 41(12):44-50. 
[Barraquand and Latombe, 1991] Barraquand, J. and Latombe, J.-C. (1991). Robot motion planning: A distributed representation approach. The International Journal of Robotics Research, 10(6):628-649.

[Bekker, 1960] Bekker, M. (1960). Off-the-road locomotion. University of Michigan Press, Ann Arbor, pages 27-29.

[Bekker, 1969] Bekker, M. G. (1969). Introduction to terrain-vehicle systems. part i: The terrain. part ii: The vehicle. Technical report, MICHIGAN UNIV ANN ARBOR.

[Bekker and Bekker, 1962] Bekker, M. G. and Bekker, M. (1962). Mechanics of off-the-road locomotion. Proceedings of the Institution of Mechanical Engineers: Automobile Division, 16(1):25-44.

[Bellman, 1957] Bellman, R. (1957). A markovian decision process. Journal of mathematics and mechanics, pages 679-684.

[Biesiadecki et al., 2006] Biesiadecki, J. J., Baumgartner, E. T., Bonitz, R. G., Cooper, B., Hartman, F. R., Leger, P. C., Maimone, M. W., Maxwell, S. A., Trebi-Ollennu, A., Tunstel, E. W., et al. (2006). Mars exploration rover surface operations: driving opportunity at meridiani planum. IEEE robotics \& automation magazine, 13(2):63-71.

[Biesiadecki et al., 2007] Biesiadecki, J. J., Leger, P. C., and Maimone, M. W. (2007). Tradeoffs between directed and autonomous driving on the mars exploration rovers. The International Journal of Robotics Research, 26(1):91-104.

[Bishop and Henkel, 1962] Bishop, A. W. and Henkel, D. J. (1962). The measurement of soil properties in the triaxial test.

[Bohlin and Kavraki, 2000] Bohlin, R. and Kavraki, L. E. (2000). Path planning using lazy prm. In Proceedings 2000 ICRA. Millennium Conference. IEEE International Conference on Robotics and Automation. Symposia Proceedings (Cat. No. 00CH37065), volume 1, pages 521-528. IEEE.

[Bonet and Geffner, 2000] Bonet, B. and Geffner, H. (2000). Planning with incomplete information as heuristic search in belief space. In Proceedings of the Fifth International Conference on Artificial Intelligence Planning Systems, pages 52-61. AAAI Press.

[Bouguelia et al., 2017] Bouguelia, M.-R., Gonzalez, R., Iagnemma, K., and Byttner, S. (2017). Unsupervised classification of slip events for planetary exploration rovers. Journal of Terramechanics, 73:95-106.

[Brown, 1992] Brown, D. (1992). Engineering and design bearing capacity of soil,(engineer manual) department of the army. US Army Corps of Engineers, Washington, DC, pages 20314-1000. 
[Bry and Roy, 2011] Bry, A. and Roy, N. (2011). Rapidly-exploring random belief trees for motion planning under uncertainty. In 2011 IEEE international conference on robotics and automation, pages 723-730. IEEE.

[Candela et al., 2017] Candela, A., Thompson, D., Dobrea, E. N., and Wettergreen, D. (2017). Planetary robotic exploration driven by science hypotheses for geologic mapping. In 2017 IEEE/RSJ International Conference on Intelligent Robots and Systems (IROS), pages 38113818. IEEE.

[Canny, 1985] Canny, J. (1985). A voronoi method for the piano-movers problem. In Proceedings. 1985 IEEE International Conference on Robotics and Automation, volume 2, pages 530-535. IEEE.

[Carpin, 2006] Carpin, S. (2006). Randomized motion planning: a tutorial. International Journal of Robotics and Automation, 21(3):184-196.

[Carrier, 2006] Carrier, W. (2006). Lunar soil simulation and trafficability parameters. Lunar Geotechnical Institute, Tech. Rep.

[Carsten et al., 2007] Carsten, J., Rankin, A., Ferguson, D., and Stentz, A. (2007). Global path planning on board the mars exploration rovers. In 2007 IEEE Aerospace Conference, pages 1-11. IEEE.

[Cassandra, 1998] Cassandra, A. R. (1998). A survey of pomdp applications. In Working notes of AAAI 1998 fall symposium on planning with partially observable Markov decision processes, volume 1724.

[Chapman, 2020] Chapman, B. (2020). Congressional committee resources on space policy during the 115th congress (2017-2018): Providing context and insight into us government space policy. Space Policy, page 101359.

[Chhaniyara et al., 2012] Chhaniyara, S., Brunskill, C., Yeomans, B., Matthews, M., Saaj, C., Ransom, S., and Richter, L. (2012). Terrain trafficability analysis and soil mechanical property identification for planetary rovers: A survey. Journal of Terramechanics, 49(2):115-128.

[Christensen et al., 2004] Christensen, P. R., Jakosky, B. M., Kieffer, H. H., Malin, M. C., McSween, H. Y., Nealson, K., Mehall, G. L., Silverman, S. H., Ferry, S., Caplinger, M., et al. (2004). The thermal emission imaging system (themis) for the mars 2001 odyssey mission. Space Science Reviews, 110(1-2):85-130.

[Collins et al., 2012] Collins, G. S., Melosh, H. J., and Osinski, G. R. (2012). The impact-cratering process. Elements, 8(1):25-30.

[Corke, 2011] Corke, P. (2011). Robotics. Vision and Control. 
[Costes et al., 1971] Costes, N., Cohron, G., and Moss, D. (1971). Cone penetration resistance test-an approach to evaluating in-place strength and packing characteristics of lunar soils. In Lunar and Planetary Science Conference Proceedings, volume 2, page 1973.

[Crisp et al., 2003] Crisp, J. A., Adler, M., Matijevic, J. R., Squyres, S. W., Arvidson, R. E., and Kass, D. M. (2003). Mars exploration rover mission. Journal of Geophysical Research: Planets, 108(E12).

[Cunningham, 2017] Cunningham, C. (2017). Improving Prediction of Traversability for Planetary Rovers Using Thermal Imaging. PhD thesis, Carnegie Mellon University.

[Das, 2007] Das, B. M. (2007). Principles of Foundation Engineering 6th Edition. Thomson.

[Diestel, 2010] Diestel, R. (2010). Graph theory. (Fifth Edition).

[Dijkstra et al., 1959] Dijkstra, E. W. et al. (1959). A note on two problems in connection with graphs. Numerische mathematik, 1(1):269-271.

[Dolgov and Thrun, 2008] Dolgov, D. and Thrun, S. (2008). Detection of principle directions in unknown environments for autonomous navigation. In Robotics: Science and Systems.

[Donald, 1987] Donald, B. R. (1987). A search algorithm for motion planning with six degrees of freedom. Artificial Intelligence, 31(3):295-353.

[Elbanhawi and Simic, 2014] Elbanhawi, M. and Simic, M. (2014). Sampling-based robot motion planning: A review. IEEE access, 2:56-77.

[Elbanhawi et al., 2013] Elbanhawi, M., Simic, M., and Jazar, R. (2013). Autonomous robots path planning: An adaptive roadmap approach. In Applied Mechanics and Materials, volume 373, pages 246-254. Trans Tech Publ.

[Eslami and Gholami, 2003] Eslami, A. A. and Gholami, A. M. (2003). Bearing capacity analysis of shallow foundations from cpt data.

[Fankhauser et al., 2018] Fankhauser, P., Bloesch, M., and Hutter, M. (2018). Probabilistic terrain mapping for mobile robots with uncertain localization. IEEE Robotics and Automation Letters, 3(4):3019-3026.

[Farritor et al., 1998] Farritor, S., Hacot, H., and Dubowsky, S. (1998). Physics-based planning for planetary exploration. In Proceedings. 1998 IEEE International Conference on Robotics and Automation (Cat. No. 98CH36146), volume 1, pages 278-283. IEEE.

[Fergason et al., 2006a] Fergason, R. L., Christensen, P. R., Bell III, J. F., Golombek, M. P., Herkenhoff, K. E., and Kieffer, H. H. (2006a). Physical properties of the mars exploration rover landing sites as inferred from mini-tes-derived thermal inertia. Journal of Geophysical Research: Planets, 111(E2). 
[Fergason et al., 2006b] Fergason, R. L., Christensen, P. R., and Kieffer, H. H. (2006b). Highresolution thermal inertia derived from the thermal emission imaging system (themis): Thermal model and applications. Journal of Geophysical Research: Planets, 111(E12).

[Ferguson and Stentz, 2005] Ferguson, D. and Stentz, A. (2005). The field d* algorithm for improved path planning and replanning in uniform and non-uniform cost environments. Robotics Institute, Carnegie Mellon University, Pittsburgh, PA, Tech. Rep. CMU-RI-TR-05-19.

[Fong et al., 2017] Fong, T., Seablom, M., Furlong, M., and Tan, F. (2017). Workshop on autonomy for future smd missions.

[Fox et al., 2016] Fox, V., Arvidson, R., Guinness, E., McLennan, S., Catalano, J., Murchie, S., and Powell, K. (2016). Smectite deposits in marathon valley, endeavour crater, mars, identified using crism hyperspectral reflectance data. Geophysical Research Letters, 43(10):4885-4892.

[Gaines et al., 2016] Gaines, D., Anderson, R., Doran, G., Huffman, W., Justice, H., Mackey, R., Rabideau, G., Vasavada, A., Verma, V., Estlin, T., et al. (2016). Productivity challenges for mars rover operations.

[Gashler et al., 2008] Gashler, M., Giraud-Carrier, C., and Martinez, T. (2008). Decision tree ensemble: Small heterogeneous is better than large homogeneous. In 2008 Seventh International Conference on Machine Learning and Applications, pages 900-905. IEEE.

[Gat et al., 1990] Gat, E., Slack, M. G., Miller, D. P., and Firby, R. J. (1990). Path planning and execution monitoring for a planetary rover. In Proceedings., IEEE International Conference on Robotics and Automation, pages 20-25. IEEE.

[Gellert et al., 2009] Gellert, R., Campbell, J., King, P., Leshin, L., Lugmair, G., Spray, J., Squyres, S., and Yen, A. (2009). The alpha-particle-X-ray-spectrometer (apsx) for the mars science laboratory (msl) rover mission. In The 40th Lunar and Planetary Science Conference, LPI Contribution, volume 1468, page 2364.

[Giacoboni, 2020] Giacoboni, N. (2020). Basic custom arduino library for hx711. https://www . mathworks.com/matlabcentral/fileexchange/ 66641-basic-custom-arduino-library-for-hx711.

[Girdhar and Dudek, 2016] Girdhar, Y. and Dudek, G. (2016). Modeling curiosity in a mobile robot for long-term autonomous exploration and monitoring. Autonomous Robots, 40(7):12671278.

[Golombek et al., 2012] Golombek, M., Huertas, A., Kipp, D., and Calef, F. (2012). Detection and characterization of rocks and rock size-frequency distributions at the final four mars science laboratory landing sites. International Journal of Mars Science and Exploration, 7:1-22. 
[Golombek et al., 2008] Golombek, M., Huertas, A., Marlow, J., McGrane, B., Klein, C., Martinez, M., Arvidson, R., Heet, T., Barry, L., Seelos, K., et al. (2008). Size-frequency distributions of rocks on the northern plains of mars with special reference to phoenix landing surfaces. Journal of Geophysical Research: Planets, 113(E3).

[Golombek et al., 2017] Golombek, M., Otero, R., Heverly, M., Ono, M., Williford, K., Rothrock, B., Milkovich, S., Almeida, E., Calef, F., Williams, N., et al. (2017). Characterization of mars rover 2020 prospective landing sites leading up to the second downselection. In Lunar and Planetary Science Conference, volume 48.

[Golombek and Rapp, 1997] Golombek, M. and Rapp, D. (1997). Size-frequency distributions of rocks on mars and earth analog sites: Implications for future landed missions. Journal of Geophysical Research: Planets, 102(E2):4117-4129.

[Golombek et al., 2003] Golombek, M. P., Haldemann, A., Forsberg-Taylor, N., Dimaggio, E. N., Schroeder, R., Jakosky, B., Mellon, M., and Matijevic, J. (2003). Rock size-frequency distributions on mars and implications for mars exploration rover landing safety and operations. Journal of Geophysical Research: Planets, 108(E12).

[Grotzinger et al., 2012] Grotzinger, J. P., Crisp, J., Vasavada, A. R., Anderson, R. C., Baker, C. J., Barry, R., Blake, D. F., Conrad, P., Edgett, K. S., Ferdowski, B., et al. (2012). Mars science laboratory mission and science investigation. Space science reviews, 170(1-4):5-56.

[Hart et al., 1968] Hart, P. E., Nilsson, N. J., and Raphael, B. (1968). A formal basis for the heuristic determination of minimum cost paths. IEEE transactions on Systems Science and Cybernetics, 4(2):100-107.

[Häselich et al., 2014] Häselich, M., Jöbgen, B., Neuhaus, F., Lang, D., and Paulus, D. (2014). Markov random field terrain classification of large-scale 3d maps. In 2014 IEEE International Conference on Robotics and Biomimetics (ROBIO 2014), pages 1970-1975. IEEE.

[Hedrick et al., 2020] Hedrick, G., Ohi, N., and Gu, Y. (2020). Terrain-aware path planning and map update for mars sample return mission. IEEE Robotics and Automation Letters, 5(4):51815188.

[Heiken et al., 1991] Heiken, G. H., Vaniman, D. T., and French, B. M. (1991). Physical properties of the lunar soil. In Lunar sourcebook-A user's guide to the Moon, pages 476-594.

[Helmick et al., 2008] Helmick, D., Angelova, A., Matthies, L., Brooks, C., Halatci, I., Dubowsky, S., and Iagnemma, K. (2008). Experimental results from a terrain adaptive navigation system for planetary rovers. In the Ninth International Symposium on Artificial Intelligence, Robotics and Automation in Space, i-SAIRAS.

[Heverly et al., 2013] Heverly, M., Matthews, J., Lin, J., Fuller, D., Maimone, M., Biesiadecki, J., and Leichty, J. (2013). Traverse performance characterization for the mars science laboratory rover. Journal of Field Robotics, 30(6):835-846. 
[Hitz et al., 2017] Hitz, G., Galceran, E., Garneau, M.-È., Pomerleau, F., and Siegwart, R. (2017). Adaptive continuous-space informative path planning for online environmental monitoring. Journal of Field Robotics, 34(8):1427-1449.

[Hollinger and Sukhatme, 2014] Hollinger, G. A. and Sukhatme, G. S. (2014). Sampling-based robotic information gathering algorithms. The International Journal of Robotics Research, 33(9):1271-1287.

[Howard et al., 2001] Howard, A., Seraji, H., and Tunstel, E. (2001). A rule-based fuzzy traversability index for mobile robot navigation. In Proceedings 2001 ICRA. IEEE International Conference on Robotics and Automation (Cat. No. 01CH37164), volume 3, pages 3067-3071. IEEE.

[Hwang et al., 1992] Hwang, Y. K., Ahuja, N., et al. (1992). A potential field approach to path planning. IEEE Transactions on Robotics and Automation, 8(1):23-32.

[Iagnemma et al., 1999] Iagnemma, K., Genot, F., and Dubowsky, S. (1999). Rapid physics-based rough-terrain rover planning with sensor and control uncertainty. In Proceedings 1999 IEEE International Conference on Robotics and Automation (Cat. No. 99CH36288C), volume 3, pages 2286-2291. IEEE.

[Iagnemma et al., 2003] Iagnemma, K., Kang, S., Brooks, C., and Dubowsky, S. (2003). Multisensor terrain estimation for planetary rovers. In Proceedings of the 8th international symposium on artificial intelligence, robotics, and automation in space. IEEE Press, New York.

[Iagnemma et al., 2004] Iagnemma, K., Kang, S., Shibly, H., and Dubowsky, S. (2004). Online terrain parameter estimation for wheeled mobile robots with application to planetary rovers. IEEE transactions on robotics, 20(5):921-927.

[Ishigami et al., 2011] Ishigami, G., Nagatani, K., and Yoshida, K. (2011). Path planning and evaluation for planetary rovers based on dynamic mobility index. In 2011 IEEE/RSJ International Conference on Intelligent Robots and Systems, pages 601-606. IEEE.

[Johnson et al., 2017] Johnson, A., Aaron, S., Chang, J., Cheng, Y., Montgomery, J., Mohan, S., Schroeder, S., Tweddle, B., Trawny, N., and Zheng, J. (2017). The lander vision system for mars 2020 entry descent and landing.

[Johnson et al., 2015] Johnson, J. B., Kulchitsky, A. V., Duvoy, P., Iagnemma, K., Senatore, C., Arvidson, R. E., and Moore, J. (2015). Discrete element method simulations of mars exploration rover wheel performance. Journal of Terramechanics, 62:31-40.

[Kavraki et al., 1996] Kavraki, L. E., Svestka, P., Latombe, J.-C., and Overmars, M. H. (1996). Probabilistic roadmaps for path planning in high-dimensional configuration spaces. IEEE transactions on Robotics and Automation, 12(4):566-580. 
[Kewlani et al., 2009] Kewlani, G., Ishigami, G., and Iagnemma, K. (2009). Stochastic mobilitybased path planning in uncertain environments. In 2009 IEEE/RSJ International Conference on Intelligent Robots and Systems, pages 1183-1189. IEEE.

[Kilic et al., 2019] Kilic, C., Gross, J. N., Ohi, N., Watson, R., Strader, J., Swiger, T., Harper, S., and $\mathrm{Gu}, \mathrm{Y}$. (2019). Improved planetary rover inertial navigation and wheel odometry performance through periodic use of zero-type constraints. arXiv preprint arXiv:1906.08849.

[Klein et al., 2014] Klein, E., Nilsen, E., Nicholas, A., Whetsel, C., Parrish, J., Mattingly, R., and May, L. (2014). The mobile mav concept for mars sample return. In 2014 IEEE Aerospace Conference, pages 1-9. IEEE.

[Koenig and Likhachev, 2002] Koenig, S. and Likhachev, M. (2002). D ${ }^{\wedge *}$ lite. Aaai/iaai, 15.

[Kschischang et al., 2001] Kschischang, F. R., Frey, B. J., and Loeliger, H.-A. (2001). Factor graphs and the sum-product algorithm. IEEE Transactions on information theory, 47(2):498519.

[Kuffner and RRT-Connect, 2000] Kuffner, J. and RRT-Connect, S. L. (2000). An efficient approach to single-query path planning. In IEEE international conference on robotics and automation, San Francisco, pages 473-479. IEEE.

[Kurniawati et al., 2012] Kurniawati, H., Bandyopadhyay, T., and Patrikalakis, N. M. (2012). Global motion planning under uncertain motion, sensing, and environment map. Autonomous Robots, 33(3):255-272.

[Kurniawati and Yadav, 2016] Kurniawati, H. and Yadav, V. (2016). An online pomdp solver for uncertainty planning in dynamic environment. In Robotics Research, pages 611-629. Springer.

[Labuz and Zang, 2012] Labuz, J. F. and Zang, A. (2012). Mohr-coulomb failure criterion. Rock mechanics and rock engineering, 45(6):975-979.

[LaValle, 1998] LaValle, S. M. (1998). Rapidly-exploring random trees: A new tool for path planning.

[LaValle, 2006] LaValle, S. M. (2006). Planning algorithms. Cambridge university press.

[Lee and Schachter, 1980] Lee, D.-T. and Schachter, B. J. (1980). Two algorithms for constructing a delaunay triangulation. International Journal of Computer \& Information Sciences, 9(3):219242.

[Lindemann and Voorhees, 2005] Lindemann, R. A. and Voorhees, C. J. (2005). Mars exploration rover mobility assembly design, test and performance. In 2005 IEEE International Conference on Systems, Man and Cybernetics, volume 1, pages 450-455. IEEE. 
[Liu et al., 2008] Liu, X.-Y., Wu, J., and Zhou, Z.-H. (2008). Exploratory undersampling for classimbalance learning. IEEE Transactions on Systems, Man, and Cybernetics, Part B (Cybernetics), 39(2):539-550.

[Lorenz et al., 2018] Lorenz, R. D., Turtle, E. P., Barnes, J. W., Trainer, M. G., Adams, D. S., Hibbard, K. E., Sheldon, C. Z., Zacny, K., Peplowski, P. N., Lawrence, D. J., et al. (2018). Dragonfly: a rotorcraft lander concept for scientific exploration at titan. Johns Hopkins APL Technical Digest, 34:374-387.

[Lozano-Pérez and Wesley, 1979] Lozano-Pérez, T. and Wesley, M. A. (1979). An algorithm for planning collision-free paths among polyhedral obstacles. Communications of the ACM, 22(10):560-570.

[Maimone et al., 2007] Maimone, M., Cheng, Y., and Matthies, L. (2007). Two years of visual odometry on the mars exploration rovers. Journal of Field Robotics, 24(3):169-186.

[Mascarich et al., 2019] Mascarich, F., Papachristos, C., Wilson, T., and Alexis, K. (2019). Distributed radiation field estimation and informative path planning for nuclear environment characterization. In 2019 International Conference on Robotics and Automation (ICRA), pages 23182324. IEEE.

[Matijevic, 1997] Matijevic, J. (1997). Sojourner the mars pathfinder microrover flight experiment.

[Mayerhof, 1976] Mayerhof, G. (1976). Bearing capacity and settlement of pile foundations. Journal of Geotechnical and Geoenvironmental Engineering, 102(ASCE\# 11962).

[McEwen et al., 2007] McEwen, A. S., Eliason, E. M., Bergstrom, J. W., Bridges, N. T., Hansen, C. J., Delamere, W. A., Grant, J. A., Gulick, V. C., Herkenhoff, K. E., Keszthelyi, L., et al. (2007). Mars reconnaissance orbiter's high resolution imaging science experiment (hirise). Journal of Geophysical Research: Planets, 112(E5).

[Melosh, 2011] Melosh, H. J. (2011). Planetary surface processes, volume 13. Cambridge University Press.

[MEPAG, 2008] MEPAG, N. D. S. A. G. (2008). Science priorities for mars sample return.

[Miller et al., 1989] Miller, D., Mishkin, A., Lambert, K., Bickler, D., and Bernard, D. (1989). Autonomous navigation and mobility for a planetary rover. In 27th Aerospace Sciences Meeting, page 859 .

[Missiuro and Roy, 2006] Missiuro, P. E. and Roy, N. (2006). Adapting probabilistic roadmaps to handle uncertain maps. In Proceedings 2006 IEEE International Conference on Robotics and Automation, 2006. ICRA 2006., pages 1261-1267. IEEE. 
[Mitchell et al., 1972] Mitchell, J., Houston, W., Scott, R., Costes, N., Carrier III, W., and Bromwell, L. (1972). Mechanical properties of lunar soil: Density, porosity, cohesion and angle of internal friction. In Lunar and Planetary Science Conference Proceedings, volume 3, page 3235 .

[Mousis et al., 2018] Mousis, O., Atkinson, D., Cavalié, T., Fletcher, L., Amato, M., Aslam, S., Ferri, F., Renard, J.-B., Spilker, T., Venkatapathy, E., et al. (2018). Scientific rationale for uranus and neptune in situ explorations. Planetary and Space Science, 155:12-40.

[Muro, 2004] Muro, T. (2004). Terramechanics: land locomotion mechanics. CRC Press.

[NASA, 2018] NASA (2018). https://www.nasa.gov/press-release/ nasa-announces-landing-site-for-mars-2020-rover.

[Nilsson, 1969] Nilsson, N. J. (1969). A mobile automaton: An application of artificial intelligence techniques. Technical report, SRI International, Menlo Park, CA - Artificial Intelligence Center.

[NYC, 2020] NYC (2014 (accessed August 26, 2020)). Civil Engineering NYC building code. https://up.codes/viewer/new_york_city/nyc-building-code-2014/ chapter/18/soils-and-foundations\#table_1804.1.

[NYT, 2020] NYT (accessed March 23, 2020). The New York Time - Mars Curiosity Rover Tracker. https://archive.nytimes.com/www.nytimes.com/interactive/science/ space/mars-curiosity-rover-tracker.html\#sol665.

[Olshen et al., 1984] Olshen, R., CJ, S., Breiman, L., and Friedman, J. (1984). Classification and regression trees.

[Ono et al., 2018] Ono, M. et al. (2018). Mars 2020 surface mission performance analysis: Part 2. surface traversability. In AIAA SPACE and Astronautics Forum and Exposition.

[Ono et al., 2016] Ono, M., Rothrock, B., Almeida, E., Ansar, A., Otero, R., Huertas, A., and Heverly, M. (2016). Data-driven surface traversability analysis for mars 2020 landing site selection. In 2016 IEEE Aerospace Conference, pages 1-12. IEEE.

[Opitz and Maclin, 1999] Opitz, D. and Maclin, R. (1999). Popular ensemble methods: An empirical study. Journal of artificial intelligence research, 11:169-198.

[Papadakis, 2013] Papadakis, P. (2013). Terrain traversability analysis methods for unmanned ground vehicles: A survey. Engineering Applications of Artificial Intelligence, 26(4):13731385.

[Paquet et al., 2006] Paquet, S., Chaib-draa, B., and Ross, S. (2006). Hybrid pomdp algorithms. In Proceedings of The Workshop on Multi-Agent Sequential Decision Making in Uncertain Domains (MSDM-06), pages 133-147. 
[Prentice and Roy, 2009] Prentice, S. and Roy, N. (2009). The belief roadmap: Efficient planning in belief space by factoring the covariance. The International Journal of Robotics Research, 28(11-12):1448-1465.

[Putzig et al., 2005] Putzig, N. E., Mellon, M. T., Kretke, K. A., and Arvidson, R. E. (2005). Global thermal inertia and surface properties of mars from the mgs mapping mission. Icarus, 173(2):325-341.

[Pyrzak et al., 2006] Pyrzak, G., McCurdy, M., Rabe, K., Norris, J., Joswig, J., Fox, J., Crockett, T., and Powell, M. (2006). Targeting and localization for mars rover operations. In 2006 IEEE International Conference on Information Reuse \& Integration, pages 23-27. IEEE.

[Rabideau and Benowitz, 2017] Rabideau, G. and Benowitz, E. (2017). Prototyping an onboard scheduler for the mars 2020 rover.

[Rahmatian and Metzger, 2010] Rahmatian, L. A. and Metzger, P. T. (2010). Soil test apparatus for lunar surfaces. In Earth and Space 2010: Engineering, Science, Construction, and Operations in Challenging Environments, pages 239-253.

[Reece, 1965] Reece, A. R. (1965). Principles of soil-vehicle mechanics. Proceedings of the Institution of Mechanical Engineers: Automobile Division, 180(1):45-66.

[Rokach, 2010] Rokach, L. (2010). Ensemble-based classifiers. Artificial intelligence review, 33(1-2):1-39.

[Rosiek et al., 2003] Rosiek, M., Howington-Kraus, E., Hare, T., and Redding, B. (2003). Mars transverse mercator $(\mathrm{mtm})$ map series updated with planetocentric grid. In $\propto$ Proceedings of the ISPRS WG IV/9 Extraterrestrial Mapping Workshop, Houston.

[Ross et al., 2008] Ross, S., Pineau, J., and Chaib-draa, B. (2008). Theoretical analysis of heuristic search methods for online pomdps. In Advances in neural information processing systems, pages $1233-1240$.

[Rothrock et al., 2016] Rothrock, B., Kennedy, R., Cunningham, C., Papon, J., Heverly, M., and Ono, M. (2016). Spoc: Deep learning-based terrain classification for mars rover missions. In AIAA SPACE 2016, page 5539.

[Russell and Santos Jr, 2019] Russell, J. and Santos Jr, E. (2019). Explaining reward functions in markov decision processes.

[Schmidt, 2012] Schmidt, M. (2012). Ugm: Matlab code for undirected graphical models. http: //www.di.ens.fr/mschmidt/Software/UGM.html.

[Schroeder, 2019] Schroeder, C. (2019). Life on mars: my 15 amazing years with oppy, nasa's record-breaking rover. 
[Seiffert et al., 2008] Seiffert, C., Khoshgoftaar, T. M., Van Hulse, J., and Napolitano, A. (2008). Rusboost: Improving classification performance when training data is skewed. In 2008 19th International Conference on Pattern Recognition, pages 1-4. IEEE.

[Seiffert et al., 2009] Seiffert, C., Khoshgoftaar, T. M., Van Hulse, J., and Napolitano, A. (2009). Rusboost: A hybrid approach to alleviating class imbalance. IEEE Transactions on Systems, Man, and Cybernetics-Part A: Systems and Humans, 40(1):185-197.

[Senatore et al., 2014] Senatore, C., Stein, N., Zhou, F., Bennett, K., Arvidson, R., Trease, B., Lindemann, R., Bellutta, P., Heverly, M., and Iagnemma, K. (2014). Modeling and validation of mobility characteristics of the mars science laboratory curiosity rover. In Proc. Proceedings of the 12th International Symposium on Artificial Intelligence, Robotics and Automation in Space (i-SAIRAS).

[Seweryn et al., 2014] Seweryn, K., Skocki, K., Banaszkiewicz, M., Grygorczuk, J., Kolano, M., Kuciński, T., Mazurek, J., Morawski, M., Białek, A., Rickman, H., et al. (2014). Determining the geotechnical properties of planetary regolith using low velocity penetrometers. Planetary and Space Science, 99:70-83.

[Shiller and Chen, 1990] Shiller, Z. and Chen, J. (1990). Optimal motion planning of autonomous vehicles in three dimensional terrains. In Proceedings. IEEE International Conference on Robotics and Automation, pages 198-203. IEEE.

[Soffen and Young, 1972] Soffen, G. A. and Young, A. T. (1972). The viking missions to mars. Icarus, 16(1):1-16.

[Stentz, 1993] Stentz, A. (1993). Optimal and efficient path planning for unknown and dynamic environments. Technical report, Carnegie-Mellon University, Pittsburgh, PA, Robotics Institute.

[Stentz et al., 1995] Stentz, A. et al. (1995). The focussed $\mathrm{d}^{\wedge *}$ algorithm for real-time replanning. In IJCAI, volume 95, pages 1652-1659.

[Sullivan et al., 2011] Sullivan, R., Anderson, R., Biesiadecki, J., Bond, T., and Stewart, H. (2011). Cohesions, friction angles, and other physical properties of martian regolith from mars exploration rover wheel trenches and wheel scuffs. Journal of Geophysical Research: Planets, 116(E2).

[Terzaghi and Peck, 1948] Terzaghi, K. and Peck, R. (1948). Soil mechanics in engineering practice: scour and scour prevention at boundaries.

[Toussaint, 2007] Toussaint, M. (2007). Bayesian inference for motion control and planning. TU, Professoren der Fak. IV.

[Warren et al., 2004] Warren, S. D., Hohmann, M. G., Auerswald, K., and Mitasova, H. (2004). An evaluation of methods to determine slope using digital elevation data. Catena, 58(3):215-233. 
[Weiss, 2004] Weiss, G. M. (2004). Mining with rarity: a unifying framework. ACM Sigkdd Explorations Newsletter, 6(1):7-19.

[Witze, 2014] Witze, A. (2014). Nasa plans mars sample-return rover. Nature, 509(7500):272.

[Wong, 2008] Wong, J. Y. (2008). Theory of ground vehicles. John Wiley \& Sons.

[Wong, 2012] Wong, J. Y. (2012). Predicting the performances of rigid rover wheels on extraterrestrial surfaces based on test results obtained on earth. Journal of Terramechanics, 49(1):49-61.

[Wong et al., 1989] Wong, J. Y. et al. (1989). Terramechanics and off-road vehicles. Elsevier.

[Yen, 1970] Yen, J. Y. (1970). An algorithm for finding shortest routes from all source nodes to a given destination in general networks. Quarterly of Applied Mathematics, 27(4):526-530.

[Zacny et al., 2010] Zacny, K., Wilson, J., Craft, J., Asnani, V., Oravec, H., Creager, C., Johnson, J., and Fong, T. (2010). Robotic lunar geotechnical tool. In Earth and Space 2010: Engineering, Science, Construction, and Operations in Challenging Environments, pages 166-181.

[Zhou et al., 2017] Zhou, F., Arvidson, R., and Zastrow, A. (2017). Simulating mars science laboratory curiosity rover traverses using artemis. In Lunar and Planetary Science Conference, volume 48 .

[Zhou et al., 2014] Zhou, F., Arvidson, R. E., Bennett, K., Trease, B., Lindemann, R., Bellutta, P., Iagnemma, K., and Senatore, C. (2014). Simulations of mars rover traverses. Journal of Field Robotics, 31(1):141-160.

[Zoback, 2010] Zoback, M. D. (2010). Reservoir geomechanics. Cambridge University Press. 


\section{Appendices}




\section{APPENDIX A \\ Terramechanics Equations}

Table A.1: Parameters used in Appendix A.

\begin{tabular}{|c|c|c|}
\hline Parameters & Description & Unit \\
\hline$b$ & Wheel width & \multirow{14}{*}{ Meters } \\
\hline$R$ & Wheel radius & \\
\hline$D$ & Wheel diameter & \\
\hline$z$ & Depth of point of interest & \\
\hline$z_{0}$ & Sinkage & \\
\hline$l(\theta)$ & Length rolled upon & \\
\hline$d(\theta)$ & Soil deformation & \\
\hline$X$ & $\begin{array}{c}\text { Coordinate of point of interest in the horizontal driving } \\
\text { direction }\end{array}$ & \\
\hline$d X$ & Elemental displacement in the $X$ direction & \\
\hline$Y$ & $\begin{array}{l}\text { Coordinate of point of interest in the vertical driving } \\
\text { directoin }\end{array}$ & \\
\hline$d Y$ & Elemental displacement in the $Y$ direction & \\
\hline$\overline{K T}$ & Elemental length of trajectory $l(\theta)$ & \\
\hline$\overline{K H}$ & $\begin{array}{l}\text { Component of } \overline{K T} \text { in the direction of the resultant between } \\
\qquad T_{d} \text { and } W\end{array}$ & \\
\hline$i_{x}$ & Shear displacement & \\
\hline$\theta$ & Angle between resultant $p$ and $\overline{K T}$ & \multirow{7}{*}{ Degrees } \\
\hline$\theta_{e}$ & $\begin{array}{l}\text { Entry angle defined by position where a point on the wheel } \\
\text { circumference first comes into contact with the terrain }\end{array}$ & \\
\hline$\xi$ & Angle between resultant $p$ and $\overline{K T}$ & \\
\hline$\epsilon$ & Angle of resultant between $T_{d}$ and $W$ to vertical axis & \\
\hline$\eta$ & Angle between $\overline{K T}$ and $R_{\omega}$ & \\
\hline$\alpha$ & $\begin{array}{l}\text { Angle between vertical radius and radius passing by the } \\
\text { point of interest }\end{array}$ & \\
\hline$\theta_{s}$ & Slope angle & \\
\hline$V$ & Longitudinal (drive direction) velocity & \multirow{4}{*}{$\begin{array}{l}\text { Meters } \\
\text { per } \\
\text { Second }\end{array}$} \\
\hline$V_{s}$ & Slip velocity & \\
\hline$\omega$ & Angular velocity & \\
\hline$n_{e}$ & Engine speed & \\
\hline$q$ & Vertical pressure & \multirow[b]{5}{*}{ Pascals } \\
\hline$\sigma_{n}$ & Normal stress & \\
\hline$\tau$ & Shear stress & \\
\hline$\tau_{\max }$ & Maximum shear stress & \\
\hline$p$ & Resultant between normal and shear stresses & \\
\hline
\end{tabular}

Continued on next page 
Table A.1 - Continued from previous page

\begin{tabular}{|c|c|c|}
\hline Parameters & Description & \multirow{2}{*}{ Unit } \\
\hline$W$ & Load & \\
\hline$T_{d}$ & Effective driving torque & \\
\cline { 1 - 2 }$q^{\prime}$ & $\begin{array}{c}\text { Component of } p(\theta) \text { to direction of } \epsilon \text { to vertical axis } \\
\text { (applied stress) }\end{array}$ & \multirow{2}{*}{ Newtons } \\
\hline$T_{e}$ & Engine torque & \\
\hline$R_{\gamma}$ & Compaction resistance & \\
\hline$F_{d}$ & Drawbar pull & \\
\hline$F$ & Thrust & \\
\hline$R_{R}$ & Sum of resisting force & \\
\hline$R_{a}$ & Aerodynamical resistance & \\
\hline
\end{tabular}

\section{A.1 Pressure and shear stress}

The pressure and shear stress are derived from plate tests [Bekker, 1960] and mapped into a cylindrical geometry [Bekker, 1969]. Shear stress is the main source of driving traction and thus the main source of slip and skid, parameters modeled in terramechanics. The equations for shear stress vs. shear displacement are first established below for shear boxes and bevameter plates experiments and then mapped into a cylindrical geometry to be applied to a wheel. The pressure $q$ is given by the Bekker-Reece pressure-sinkage equation assuming a homogeneous soil under a plate of width $b$ [Reece, 1965]. This equation is inspired by Terzaghi's bearing capacity theory [Wong, 2012]:

$$
q=\left(c k_{c}^{\prime}+\gamma b k_{\phi}^{\prime}\right)\left(\frac{z_{0}}{b}\right)^{n}
$$

where $k_{c}$ ' is the Reece cohesion modulus (unitless), $k_{\phi}{ }^{\prime}$ is the Reece friction modulus (unitless), $\gamma$ is the soil weight density $\left(\mathrm{N} / \mathrm{m}^{3}\right), n$ is the pressure-sinkage exponent, $c$ is cohesion $(\mathrm{kPa})$ and $z_{0}$ is sinkage $(m)$. Locally, a wheel can be approximated by a plate at the point of application of pressure and the above equation is thus applied for a wheel geometry. The shear stress - shear displacement relationship for a plastic soil is given by Janosi (1961):

$$
\tau=\tau_{\max }\left(1-\exp \left(\frac{j_{x}}{k_{x}}\right)\right)
$$

where $j_{x}$ is the soil shear displacement, $k_{x}$ is the longitudinal shear deformation modulus and $\tau_{\max }$ is the maximum shear stress possible before failure of the soil, given by the Mohr-Coulomb criterion:

$$
\tau_{\max }=c+\sigma_{n} \tan \phi
$$

where $c$ is the cohesion, $\sigma_{n}$ is the normal stress and $\phi$ is the angle of internal friction. In the case of a wheel, the shear stress at the interface between the wheel and the soil is a function of the soil 


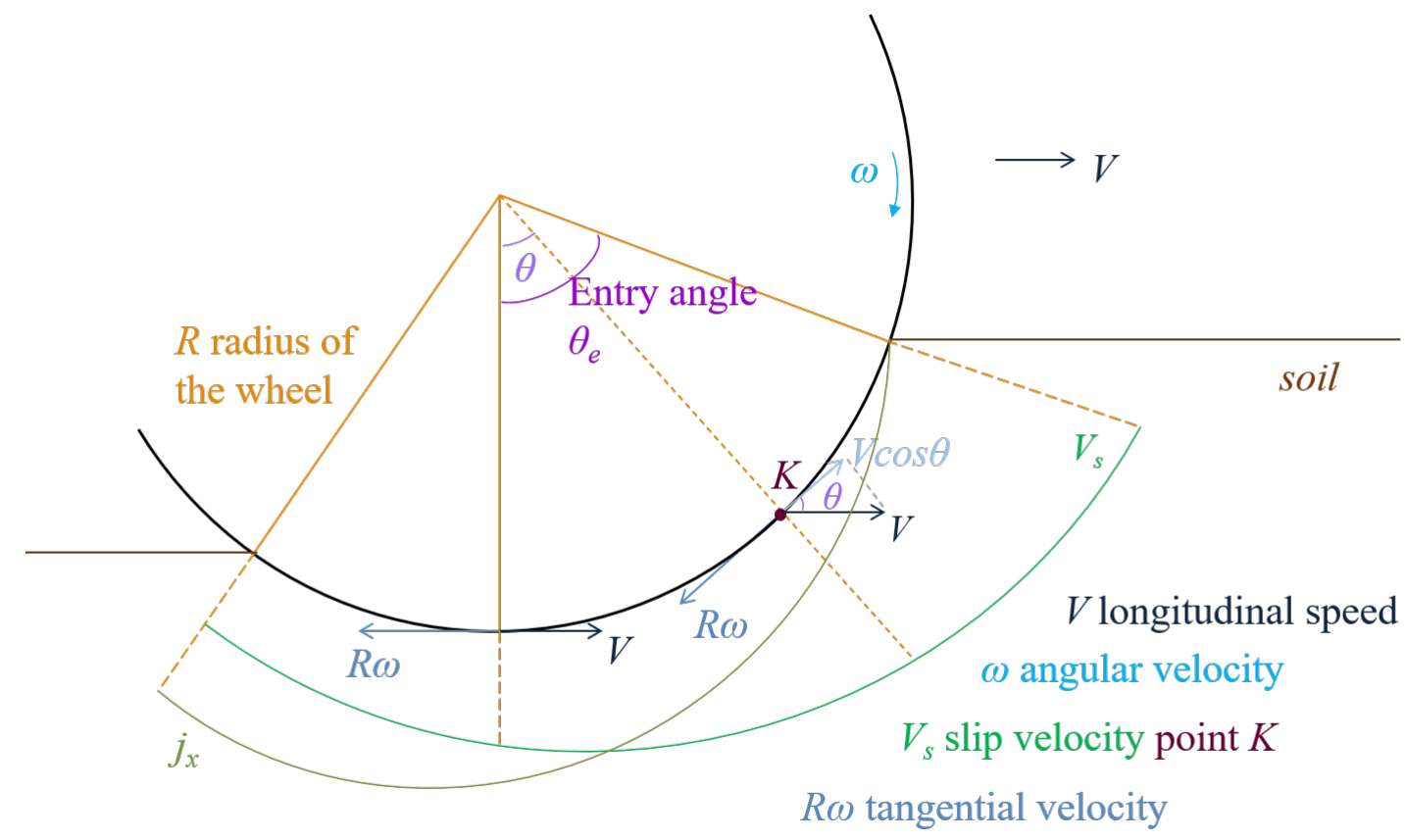

$j_{x}$ soil shear displacement

Figure A.1: Driven wheel with velocities and forces applied on a given point $K$ on the circumference of the wheel. $\theta$ is the angle between the dead center of the wheel and the point of study and $\theta_{e}$ is the entry angle defined by the position where a point on the wheel circumference first comes into contact with the terrain, which is related to sinkage (a high entry angle means high sinkage and vice versa) [Wong, 2008].

shear displacement [Wong, 2008] derived for cylindrical geometry. The soil shear displacement derivation is based on the slip velocity $\left(V_{s}\right)$ analysis on the wheel-soil interface as shown in Fig.A.1. There are two cases that need to be distinguished: the wheel slipping and the wheel skidding. The soil shear displacement is defined as follows:

$$
j_{x}=\int_{0}^{t} V_{s} d t
$$

where $V_{s}$ is the slip velocity given by Eq. A.5 and illustrated by Fig.A.2.

$$
V_{s}=R \omega-V \cos \theta
$$

is the angle between the studied point $K$ of application of forces and the dead center of the wheel. Slip and skid for a wheel are given by Eq. A.6 and A.7, respectively:

$$
\begin{aligned}
& i=1-\frac{V}{R \omega} \\
& i_{s}=1-\frac{R \omega}{V}
\end{aligned}
$$

From Fig.A.2 the soil shear displacements for slip and skid patterns are derived, starting with the longitudinal speed $V$ (velocity in the drive direction) given by Eq. A.8 and A.9 (case slip and skid respectively): 


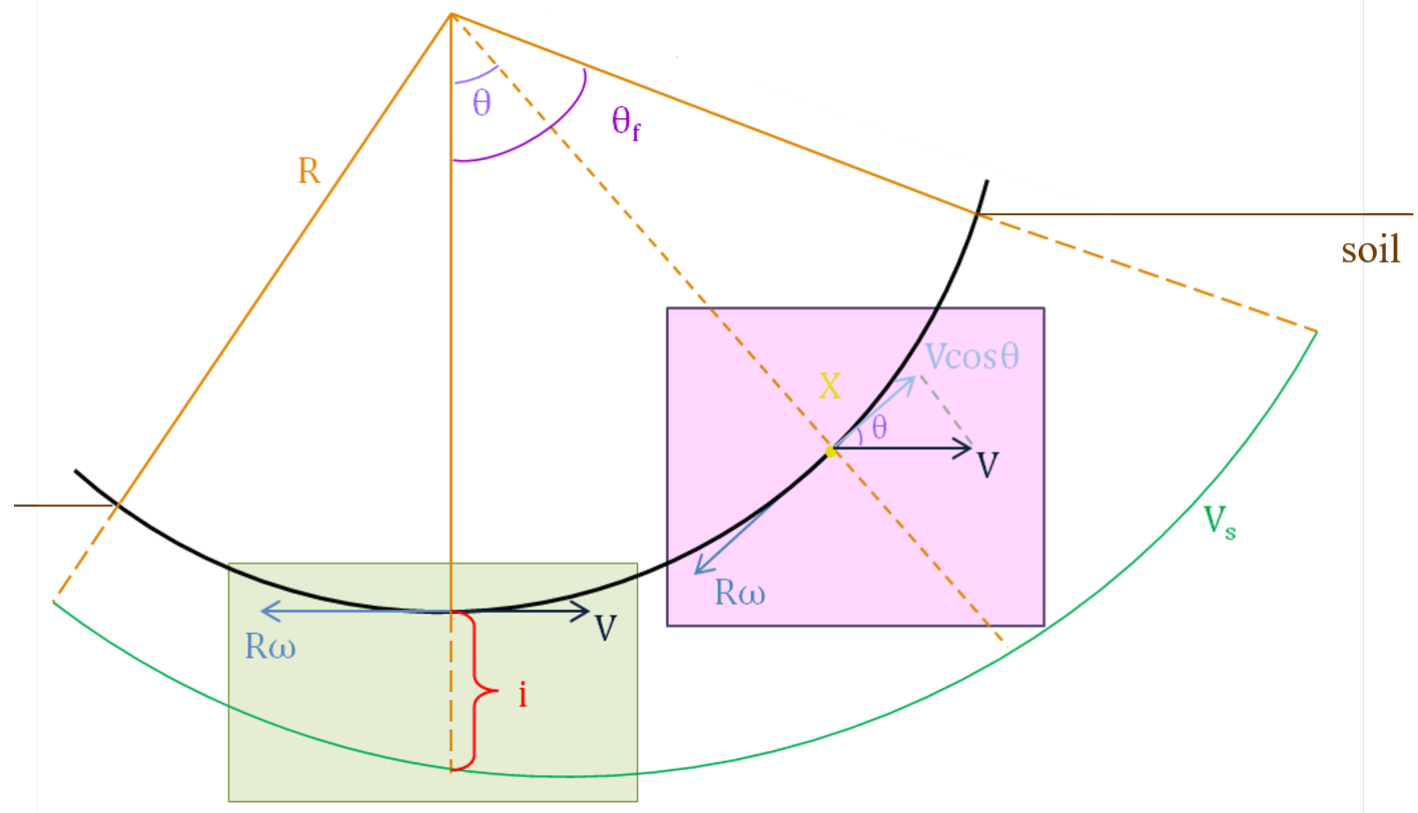

Figure A.2: Simplified scheme of the driven wheel showing slip velocity and slip $i$.

$$
\begin{gathered}
V_{s}=R \omega-R \omega \cos (\theta)(1-i) \Longleftrightarrow V_{s}=R \omega(1-\cos \theta(1-i)) \\
V_{s}=R \omega-\cos (\theta) \frac{R \omega}{1-i_{s}} \Longleftrightarrow V_{s}=R \omega\left(1-\frac{\cos \theta}{1-i_{s}}\right)
\end{gathered}
$$

$\theta$ is the angle between the dead center of the wheel and the point of study, and $\theta_{e}$ is the entry angle defined by the position where a point on the wheel circumference first come into contact with the terrain, which is related to sinkage: a high entry angle means high sinkage and vice versa. Equations A.8 and A.9 are put into Eq. A.4:

$$
\begin{gathered}
j_{x}=\int_{0}^{t} V_{s} d t \Longleftrightarrow j_{x}=\int_{0}^{t} R \omega(1-\cos \theta(1-i)) d t \\
j_{x}=\int_{0}^{t} V_{s} d t \Longleftrightarrow j_{x}=d t
\end{gathered}
$$

Moreover:

$$
\omega=\frac{d \theta}{d t}
$$

Equation A.12 is put into Eq. A.10 to get the shear soil displacement for a wheel slipping through soil [Wong, 2008]:

$$
\begin{aligned}
j_{x} & =R \int_{\theta}^{\theta_{f}} 1-\cos (\theta)(1-i) d \theta \\
& =R \int_{\theta}^{\theta_{f}} d \theta-R(i-1) \int_{\theta}^{\theta_{f}} \cos \theta d \theta \\
& =R\left(\left(\theta-\theta_{f}\right)-(1-i)\left(\sin \theta_{f}-\sin \theta\right)\right)
\end{aligned}
$$


The same replacement is done with Eq. A.11 to obtain the shear soil displacement in case of wheel skid [Wong, 2008]:

$$
\begin{aligned}
j_{x} & =R \int_{\theta}^{\theta_{f}} 1-\cos (\theta) \frac{R}{1-i_{s}} d \theta \\
& =R \int_{\theta}^{\theta_{f}} d \theta-\frac{R}{\left(1-i_{s}\right)} \int_{\theta}^{\theta_{f}} \cos \theta d \theta \\
& =R\left(\left(\theta-\theta_{f}\right)-\frac{\sin \theta_{f}-\sin \theta}{1-i_{s}}\right)
\end{aligned}
$$

The shear soil displacement depends on the size of the wheel and the entry angle, i.e. sinkage.

\section{A.2 Soil deformation under a driven wheel}

Under a driven wheel the soil experiences deformation that can be measured at a given point $K$ [Muro, 2004]. The soil deformation $d(\theta)$ is the length of the trajectory $l(\theta)$ in the direction of applied stress $q^{\prime}(\theta)$, the component of resultant stress $p(\theta)$ to the direction of resultant of load $W$ and driving torque $T_{d}$ (Fig.A.3). $\overline{K T}$ is an elemental length of trajectory of $l(\theta)$ directed in the same direction as the resultant velocity vector of the vehicle velocity $V$ and the circumferential speed $R \omega . \overline{K H}$ is the component of $\overline{K T}$ in the direction of the angle of driving torque $T_{d}$ to load $W$ (Fig.A.4 (a)).

$V$ represents longitudinal velocity, $R$ radius of the wheel, $\omega$ angular velocity, $\tau$ shear stress at point $K$, and $\sigma_{n}$ normal stress at point $K . d(\theta)$ is derived, $\theta$ being the angle between the dead center of the wheel and point $K$, defined as follows [Muro, 2004]:

$$
d(\theta)=\int_{\theta}^{\theta_{f}} \overline{K H} d \theta
$$

From the geometry in Fig.A.4 (b), $d(\theta)$ is retrieved:

$$
d(\theta)=\int_{\theta}^{\theta_{f}} \overline{K T \cos (\beta) d \theta}
$$

and Fig.A.4 (c) gives:

$$
d(\theta)=\int_{\theta}^{\theta_{f}} \overline{K T} \cos (90-(\theta+\zeta+\eta)) d \theta \Longleftrightarrow d(\theta)=\int_{\theta}^{\theta_{f}} \overline{K T} \sin (\theta+\zeta+\eta) d \theta
$$

$\epsilon$ is the angle between the resultant driving torque $T_{d}$ and the load $W$ (Fig.A.4), and $\eta$ is the angle between $\overline{K T}$ and $R \omega$. Let $G(X, Y)$ be the location of an arbitrary point on a wheel slipping through soil, which drives $l(\theta)$ in a plane $(X, Y)$ as defined in Fig.A.5 (Wong, 2001). $d X$ and $d Y$ are thus elemental displacement in the $X$ and $Y$ direction of the driven wheel. $X$ and $Y$ are given by the following equations:

$$
X=R(\alpha(1-i)+\sin \alpha)
$$




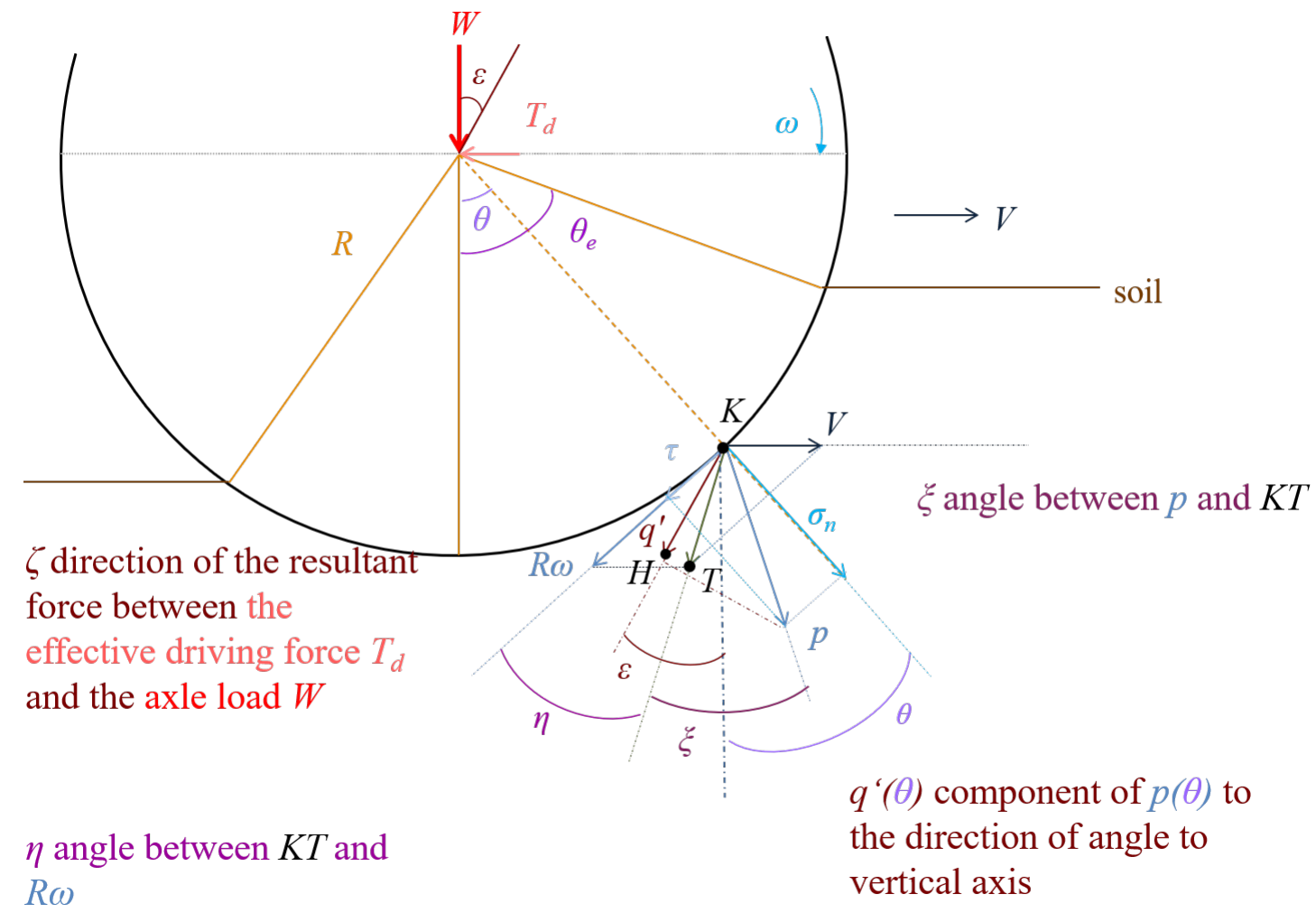

Figure A.3: Driven wheel with forces and stresses applied on a given point $K$ on the circumference. The angles between the different stresses and forces are given in the figure [Muro, 2004].
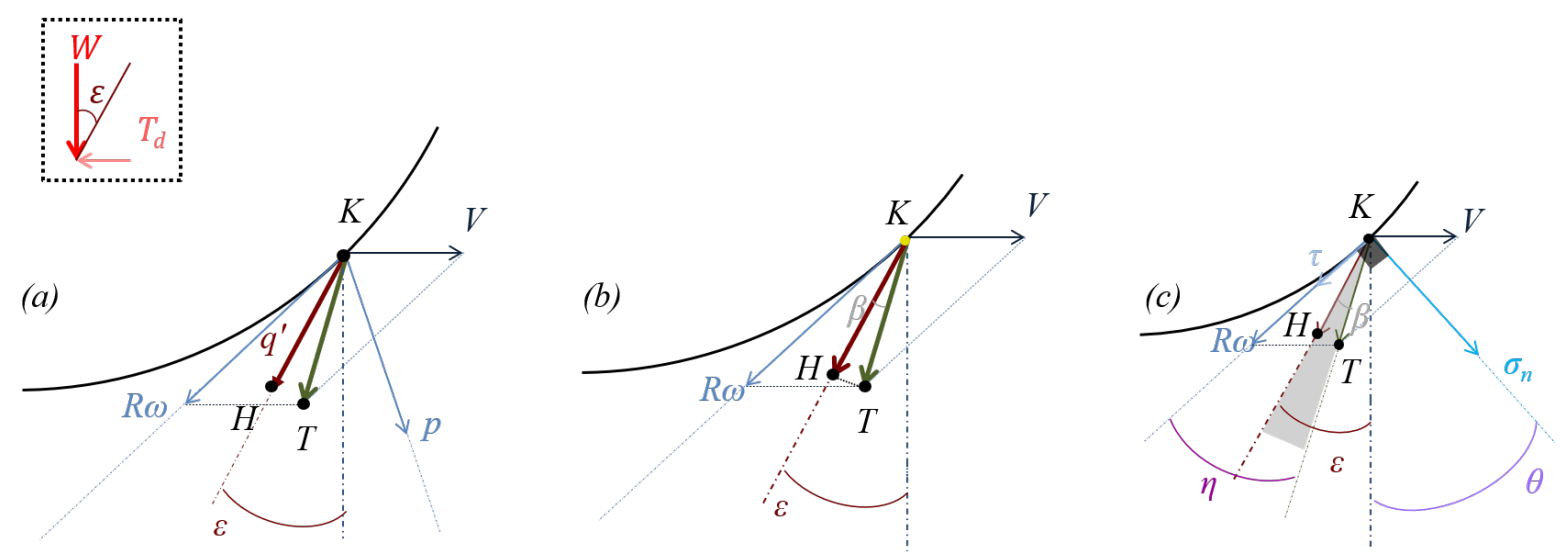

Figure A.4: Enlarged area from Fig.A.3 showing: (a) vectors $\overline{K T}$ and $\overline{K H}$, (b) angle between vectors and stresses and (c) shear and normal stresses added to the other stresses at a given point on the wheel [Muro, 2004]. 


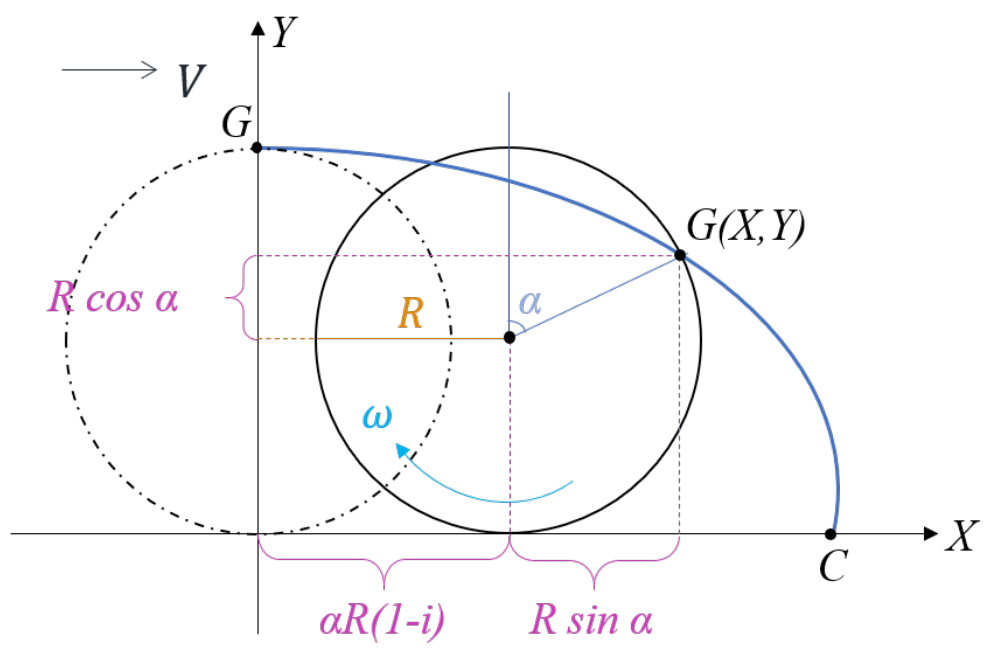

Figure A.5: Wheel driving and motion on a $(X, Y)$ plane at a given point on the circumference, referred to as $G(X, Y)$. This point touches the ground at point $C, V$ is the longitudinal velocity and $R$ the radius of the wheel [Muro, 2004].

$$
Y=R(1+\cos \alpha)
$$

where $\alpha$ is the angle between the vertical radius and the radius passing by the studied point $G(X, Y)$ and $i$ is the wheel slip. The length driven by the wheel at point $G$ is thus:

$$
l(\theta)=\sqrt{d X^{2}+d Y^{2}}=\sqrt{1+\left(\frac{d X}{d Y}\right)^{2} d X}
$$

And from Eq. A.18 and A.19, $d X$ and $d Y$ are retrieved for a wheel slipping through soil:

$$
\begin{gathered}
d X=d(R(\alpha(1-i)+\sin \alpha))=(1-i)+\cos \alpha \\
d Y=d(R(1+\cos \alpha))=-R \sin \alpha
\end{gathered}
$$

Hence:

$$
\frac{d X}{d Y}=-\frac{\sin \alpha}{(1-i)+\cos \alpha}
$$

This is put in Eq. A.20 as well as Eq. A.21 and A.22:

$$
l(\theta)=\int_{\alpha}^{\alpha_{f}}\left(\sqrt{1+\left(\frac{\sin \alpha}{R(1-i)+\cos \alpha}\right)^{2}} R(1-i+\cos \alpha) d \alpha\right.
$$

From the geometry:

$$
\begin{array}{r}
\alpha=\pi-\theta \\
\alpha_{f}=\pi-\theta_{f} \\
\cos \alpha=\cos (\pi-\theta)=\cos \theta \\
\sin \alpha=\sin (\pi-\theta)=-\sin \theta
\end{array}
$$


Thus Eq. A.24 is rewritten as follows:

$$
\begin{aligned}
l(\theta) & =\int_{\theta_{f}}^{\theta}\left(\sqrt{1+\left(\frac{\sin \theta}{1-i+\cos \theta}\right)^{2}} R(1-i+\cos \theta)\right) d \theta \\
& =R \int_{\theta_{f}}^{\theta} \sqrt{(1-i+\cos \theta)^{2}+(\sin \theta)^{2}} d \theta \\
& =R \int_{\theta_{f}}^{\theta} \sqrt{(1-i)^{2}+2(1-i) \cos \theta+1} d \theta
\end{aligned}
$$

Equation A.26 is put into Eq. A.21 and A.22, given that $\overline{K T}$ is an elemental length of trajectory of $l(\theta)$ :

$$
d(\theta)=\int_{\theta}^{\theta_{f}} \overline{F T} \sin (\theta+\zeta+\eta)=R \int_{\theta_{f}}^{\theta}\left(\sqrt{(1-i)^{2}+2(1-i) \cos \theta+1}\right) \sin (\theta+\zeta+\eta) d \theta
$$

The angle $\eta$ can be retrieved from the geometry as shown in Fig.A.6:

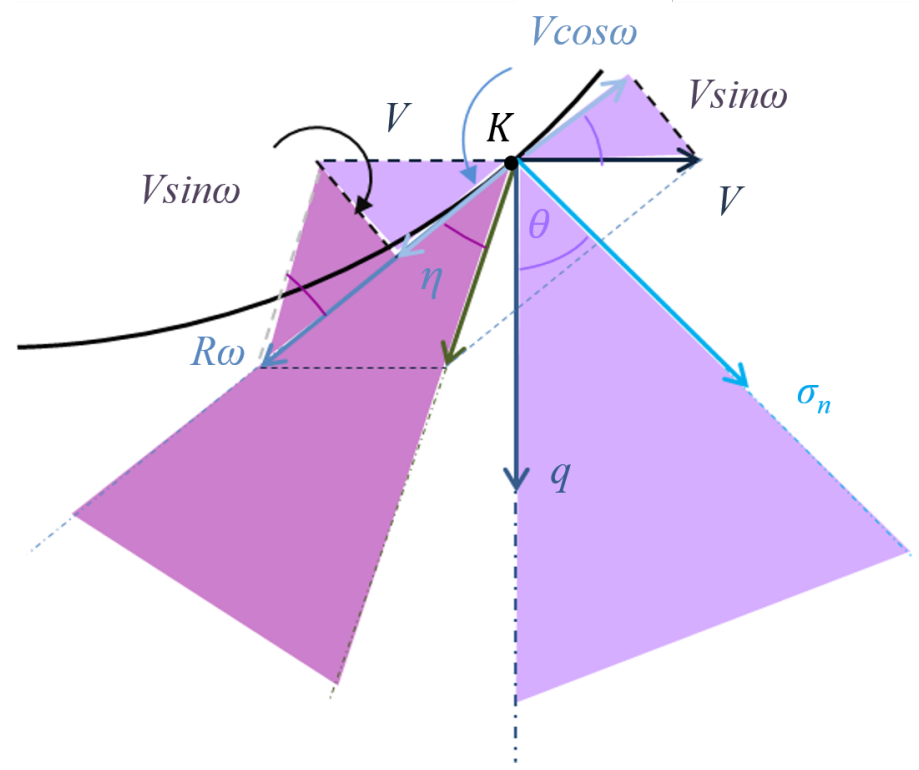

Figure A.6: Enlarged area from Fig.A.3 at point $K$ giving the geometry of $\tan \eta$.

$$
\begin{aligned}
\tan \eta & =\frac{(1-i) \sin \theta}{R \omega-V \cos \theta} \\
& =\frac{(1-i) \sin \theta}{1-(1-i) \cos \theta}
\end{aligned}
$$


Equations A.28 is put into Eq. A.27, giving the soil deformation in case of slip:

$$
d(\theta)=R \int_{\theta_{f}}^{\theta}\left(\sqrt{(1-i)^{2}+2(1-i) \cos \theta+1}\right) \sin \left(\theta+\zeta+\tan ^{-1} \frac{(1-i) \sin \theta}{1-(1-i) \cos \theta}\right) d \theta
$$

The same derivation can be made in case of wheel skid, giving the following expression for the soil deformation [Muro, 2004]:

$$
d(\theta)=R \int_{\theta_{f}}^{\theta}\left(\sqrt{\left(\frac{1}{1-i}\right)^{2}+2\left(\frac{1}{1-i}\right) \cos \theta+1}\right) \sin \left(\theta+\zeta+\tan ^{-1}\left(\frac{\sin \theta}{1-i-\cos \theta}\right)\right) d \theta
$$

As well as the soil shear displacement, the soil deformation is a function of the size of the wheel (dependence on radius $R$ ) and how much contact the wheel makes with the terrain underneath (dependence on angle $\theta$ ).

\section{A.3 Compaction resistance}

A wheel creates compaction resistance $R_{c}$ from the work done on compacting soil [Wong, 2008]. From the previous section the pressure exerted by the wheel on the surface at a given point $K$ is known:

$$
\begin{array}{r}
q=k_{e q} z^{n} \\
k_{e q}=\frac{1}{b^{n}}\left(c k_{c}^{\prime}+\gamma b k_{\phi}^{\prime}\right)
\end{array}
$$

with $k_{c}^{\prime}$ and $k_{\phi}^{\prime}$ the Reece moduli defined earlier, $n$ the pressure-sinkage exponent, $c$ cohesion and $\gamma$ the soil weight density. Let $L$ be the work per unit area required to compress the soil:

$$
L=\frac{R_{c} l}{b l}
$$

with $l$ the distance rolled upon. $L$ is the integration of force over distance, as follows [Muro, 2004]:

$$
L=\int_{0}^{z_{0}} q d z=\int_{0}^{z_{0}} k_{e q} z^{n} d z=k_{e q} \frac{z_{0}^{n+1}}{n+1}
$$

where $z_{0}$ is depth of sinkage, $q$ is pressure, $b$ is the wheel width, $D$ its diameter, $l$ the distance rolled upon, $W$ the load and on is normal stress at a given point $K$ (Fig.A.7). From Eq. A.31 and Eq. A.33 an expression for the compaction resistance is retrieved [Wong, 2008]:

$$
R_{c}=L b \Longleftrightarrow R_{c}=k_{e q} b \frac{z_{0}^{n+1}}{n+1}
$$

The equation is then rearranged. The load applied to a wheel can be written as follows:

$$
W=b \int_{0}^{\theta_{f}} R \sigma_{n} \cos \theta d \theta
$$




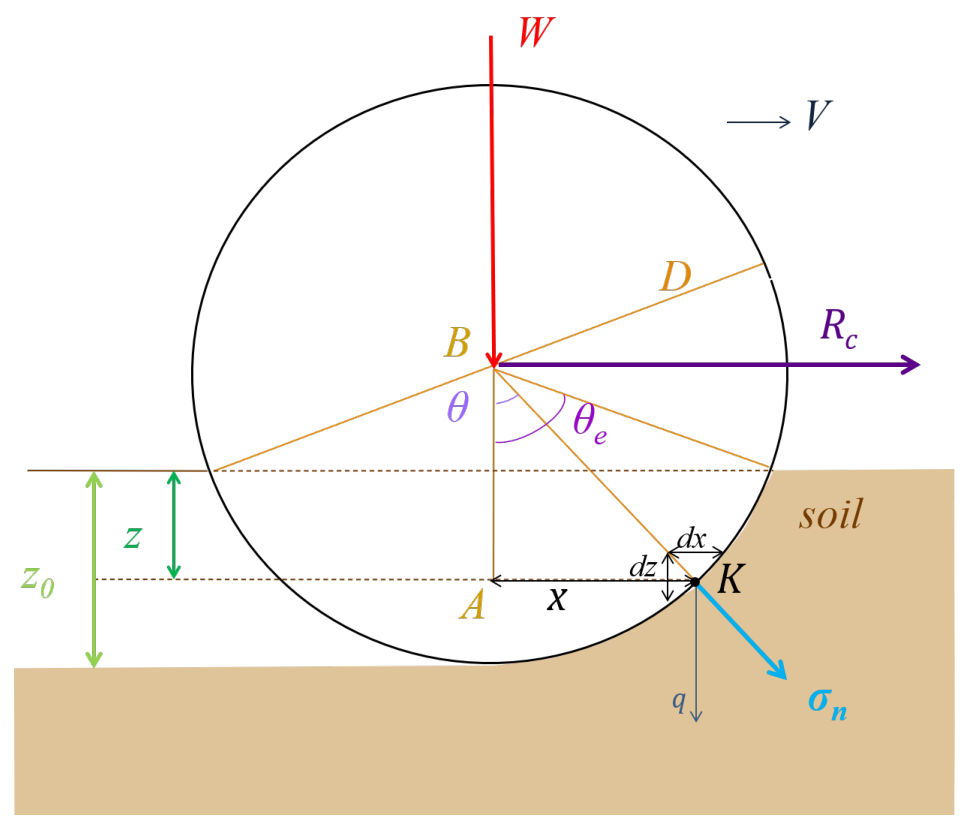

Figure A.7: Compaction resistance $R_{c}$ shown for a driven wheel with the normal stress $\sigma_{n}$, sinkage $z_{0}$, and load $W$ for a driven wheel of diameter $D . d x$ and $d z$ are elements of contact area between wheel and soil at a studied point $K$ at a depth $z, q$ is vertical pressure, $B$ is the center of the wheel and $A$ is the vertical projection of $B$ at depth $z$. $V$ is the longitudinal velocity, $\Theta$ is the angle between the bottom of the wheel and point $K$ and $\theta_{e}$ is the entry angle [Bekker, 1969].

From Fig.A.8:

$$
R \sigma_{n} \cos \theta d \theta=-q d x
$$

where $d x$ is an element of contact area between wheel and ground. Thus:

$$
W=\int_{0}^{z_{0}}-q b d x=-\int_{0}^{z_{0}} b k_{e q} z^{n} d x
$$

From the geometry shown in Fig.A.8 and using the Pythagorean Theorem:

$$
\begin{gathered}
\overline{A B}=\frac{D}{2}-\left(z_{0}-z\right) \\
x^{2}=\left(\frac{D}{2}\right)^{2}-(\overline{A B})^{2}
\end{gathered}
$$




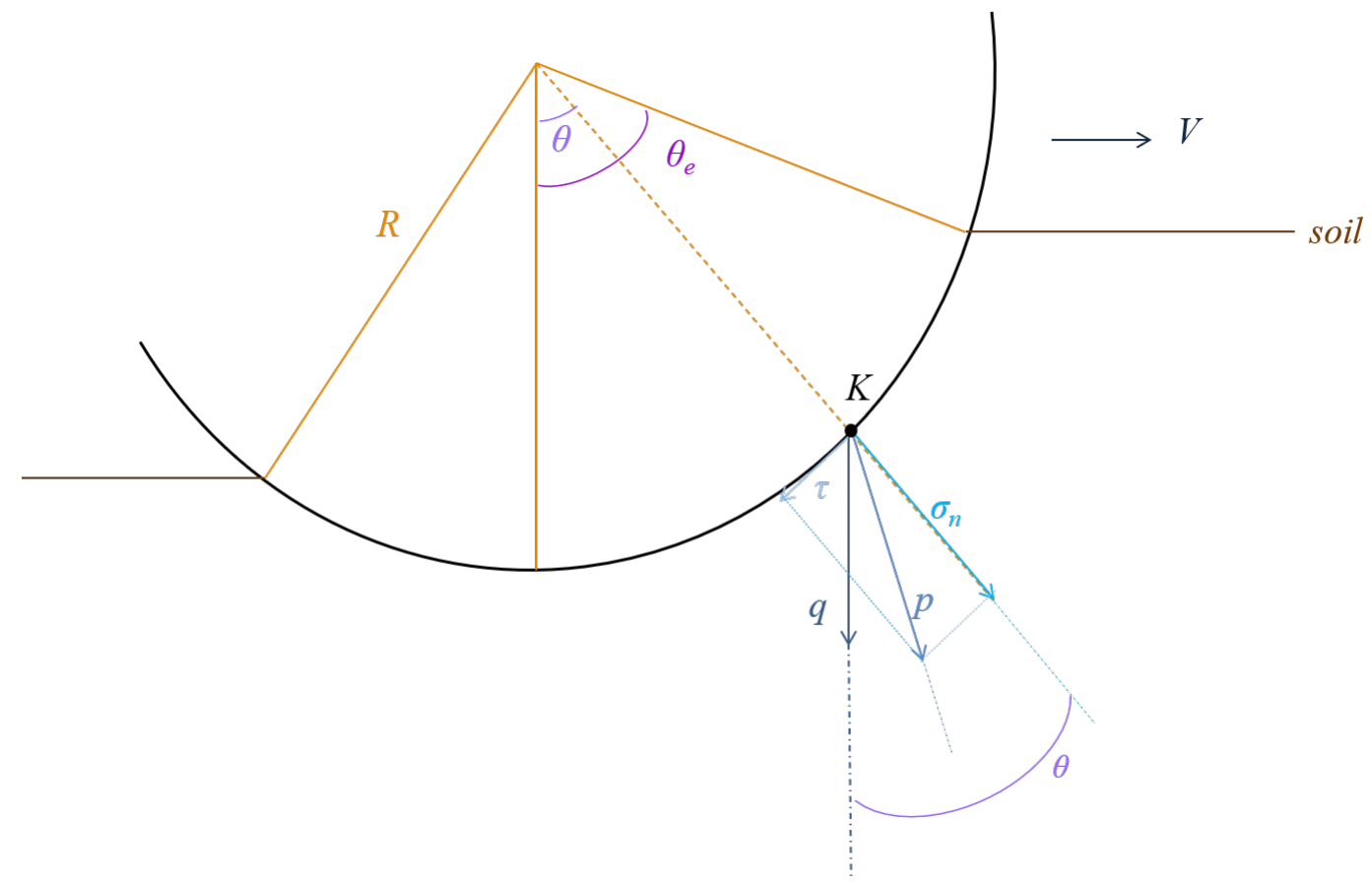

Figure A.8: Stresses and forces at point $K$ on the circumference of a driven wheel [Muro, 2004].

Thus:

$$
\begin{aligned}
x^{2} & =\left(\frac{D}{2}\right)^{2}-\left(\frac{D}{2}-\left(z_{0}-z\right)\right)^{2} \\
& =\left(\frac{D}{2}\right)^{2}-\left(\left(\frac{D}{2}\right)^{2}-2 \frac{D}{2}\left(z_{0}-z\right)+\left(z_{0}-z\right)^{2}\right) \\
& =D\left(z_{0}-z\right)-\left(z_{0}-Z\right)^{2} \\
& =\left(z_{0}-z\right)\left(D-\left(z_{0}-z\right)\right) \\
& =D\left(z_{0}-z\right) \text { for }\left(z_{0}-z\right) \text { small }
\end{aligned}
$$

Differentiating the above equation gives:

$$
2 x d x=-D d z \Longleftrightarrow d x=\frac{-D d z}{2 x}
$$

that is put into Eq. A.35 along with Eq. A.31:

$$
W=-\int_{0}^{z_{0}} b k_{e q} z^{n} d x
$$


A variable substitution is made in the above equation. Let $t^{2}=z_{0}-z$. Then $d z=-2 t d t$. Thus:

$$
\begin{aligned}
W & =b k_{e q} \int_{0}^{z_{0}} z^{n} \frac{\sqrt{D}}{2 \sqrt{z_{0}-z}} d z \\
& =b k_{e q} \int_{t=0}^{t=\sqrt{z_{0}}}\left(z_{0}-t^{2}\right)^{n} \frac{\sqrt{D}}{2 t} d z \\
& =b k_{e q} \sqrt{D} \int_{t=0}^{t=\sqrt{z_{0}}}\left(z_{0}-t^{2}\right) d t
\end{aligned}
$$

A Taylor development gives:

$$
\left(z_{0}-t^{2}\right)^{n} \approx\left(z_{0}^{n}-n z_{0}^{n-1} t^{2}+\ldots\right)
$$

Hence:

$$
\begin{aligned}
W & =b k_{e q} \sqrt{D} \int_{t=0}^{t=\sqrt{z_{0}}}\left(z_{0}^{n}-n z_{0}^{n-1} t^{2}\right) d z \\
& =b k_{e q} \frac{3-n}{3} \sqrt{D z_{0}} z_{0}^{n}
\end{aligned}
$$

Rearranging the above equation an expression for sinkage $z_{0}$ is retrieved:

$$
z_{0}^{\frac{2 n+1}{2}}=\frac{3 W}{b k_{e q}(3-n) \sqrt{D}} \Longleftrightarrow z_{0}=\left(\frac{3 W}{b k_{e q}(3-n) \sqrt{D}}\right)^{\frac{2}{2 n+1}}
$$

Sinkage given in Eq. A.45 is replaced in the relationship giving the compaction resistance (Eq. A.34) [Wong, 2008]:

$$
\begin{aligned}
R_{c} & =k_{e q} b \frac{Z_{0}^{n+1}}{n+1} \\
& =k_{e q} b \frac{\left(\frac{3 W}{b k_{e q}(3-n) \sqrt{D}}\right)^{\frac{2 n+2}{2 n+1}}}{n+1} \\
& =\frac{1}{(n+1)(3-n)^{\frac{2 n+2}{2 n+1}}\left(\frac{c k_{c}^{\prime}+\gamma b k_{\phi}^{\prime}}{b^{n-1}}\right)^{\frac{1}{2 n+1}}}\left(\frac{3 W}{\sqrt{D}}\right)^{\frac{2 n+2}{2 n+1}}
\end{aligned}
$$

Load and compaction resistance (thus load and sinkage) are related, and the equation reflects the intuitive statement that the higher the load is, the more sinkage the wheel creates. 


\section{APPENDIX B \\ Probability Mass Functions of velocity categories}

The PMFs of velocity categories matrices under CFA and slope conditions are presented in Tables B.1 through B.11. The edge potential matrices can be derived from the PMFs matrices by normalizing the columns.

Table B.1: CFA $\leq 7 \%$ and slope $\leq 10^{\circ}$.

\begin{tabular}{|c|c|c|c|c|c|c|c|}
\cline { 3 - 8 } \multicolumn{2}{c|}{} & \multicolumn{6}{c|}{ Prediction } \\
\cline { 3 - 8 } \multicolumn{2}{c|}{} & $\mathrm{A}$ & $\mathrm{B}$ & $\mathrm{C}$ & $\mathrm{D}$ & $\mathrm{E}$ & $\mathrm{F}$ \\
\hline \multirow{3}{*}{} & $\mathrm{A}$ & $84.0 \%$ & $0 \%$ & $7.50 \%$ & $.200 \%$ & $0 \%$ & $0 \%$ \\
\cline { 2 - 8 } & $\mathrm{B}$ & $0 \%$ & $0 \%$ & $0 \%$ & $0 \%$ & $0 \%$ & $0 \%$ \\
\cline { 2 - 8 } & $\mathrm{C}$ & $10.5 \% \mathrm{~S}$ & $0 \%$ & $81.3 \%$ & $1.20 \%$ & $0 \%$ & $.200 \%$ \\
\cline { 2 - 8 } & $\mathrm{D}$ & $5.20 \%$ & $0 \%$ & $10.6 \%$ & $98.4 \%$ & $0 \%$ & $0 \%$ \\
\cline { 2 - 8 } & $\mathrm{E}$ & $0 \%$ & $0 \%$ & $0 \%$ & $0 \%$ & $0 \%$ & $0 \%$ \\
\cline { 2 - 8 } & $\mathrm{F}$ & $.300 \%$ & $0 \%$ & $.600 \%$ & $.200 \%$ & $0 \%$ & $99.8 \%$ \\
\hline \multirow{2}{*}{} & & & & & & & \\
\hline
\end{tabular}

Table B.2: CFA $\leq 7 \%$ and $10^{\circ}<$ slope $\leq 15^{\circ}$.

\begin{tabular}{|c|c|c|c|c|c|c|c|}
\cline { 3 - 7 } \multicolumn{2}{c|}{} & \multicolumn{6}{c|}{ Prediction } \\
\cline { 3 - 8 } \multicolumn{2}{c|}{} & $\mathrm{A}$ & $\mathrm{B}$ & $\mathrm{C}$ & $\mathrm{D}$ & $\mathrm{E}$ & $\mathrm{F}$ \\
\hline \multirow{3}{*}{} & $\mathrm{A}$ & $84.0 \%$ & $0 \%$ & $7.50 \%$ & $0 \%$ & $0 \%$ & $.0872 \%$ \\
\cline { 2 - 8 } & $\mathrm{B}$ & $0 \%$ & $0 \%$ & $0 \%$ & $0 \%$ & $0 \%$ & $0 \%$ \\
\cline { 2 - 8 } & $\mathrm{C}$ & $10.5 \%$ & $0 \%$ & $83.3 \%$ & $0 \%$ & $0 \%$ & $.636 \%$ \\
\cline { 2 - 8 } & $\mathrm{D}$ & $0 \%$ & $0 \%$ & $0 \%$ & $0 \%$ & $0 \%$ & $0 \%$ \\
\cline { 2 - 8 } & $\mathrm{E}$ & $0 \%$ & $0 \%$ & $0 \%$ & $0 \%$ & $0 \%$ & $0 \%$ \\
\cline { 2 - 8 } & $\mathrm{F}$ & $2.44 \%$ & $0 \%$ & $4.96 \%$ & $0 \%$ & $0 \%$ & $99.2 \%$ \\
\hline
\end{tabular}

Table B.3: CFA $\leq 7 \%$ and $15^{\circ}<$ slope $\leq 20^{\circ}$.

\begin{tabular}{|c|c|c|c|c|c|c|c|}
\cline { 3 - 8 } \multicolumn{2}{c|}{} & \multicolumn{6}{c|}{ Prediction } \\
\cline { 3 - 8 } \multicolumn{2}{c|}{} & $\mathrm{A}$ & $\mathrm{B}$ & $\mathrm{C}$ & $\mathrm{D}$ & $\mathrm{E}$ & $\mathrm{F}$ \\
\hline \multirow{3}{*}{} & $\mathrm{A}$ & $0 \%$ & $0 \%$ & $0 \%$ & $0 \%$ & $0 \%$ & $0 \%$ \\
\cline { 2 - 8 } & $\mathrm{B}$ & $0 \%$ & $84.0 \%$ & $7.50 \%$ & $0 \%$ & $0 \%$ & $.0872 \%$ \\
\cline { 2 - 8 } & $\mathrm{C}$ & $0 \%$ & $10.5 \%$ & $81.3 \%$ & $0 \%$ & $0 \%$ & $.636 \%$ \\
\cline { 2 - 8 } & $\mathrm{D}$ & $0 \%$ & $0 \%$ & $0 \%$ & $0 \%$ & $0 \%$ & $0 \%$ \\
\cline { 2 - 8 } & $\mathrm{E}$ & $0 \%$ & $0 \%$ & $0 \%$ & $0 \%$ & $0 \%$ & $0 \%$ \\
\cline { 2 - 8 } & $\mathrm{F}$ & $0 \%$ & $2.44 \%$ & $4.96 \%$ & $0 \%$ & $0 \%$ & $99.2 \%$ \\
\hline \multirow{2}{*}{} & & & & & & & \\
\hline
\end{tabular}


Table B.4: CFA $\leq 7 \%$ and $20^{\circ}<$ slope $\leq 25^{\circ}$.

\begin{tabular}{|c|c|c|c|c|c|c|c|}
\cline { 3 - 7 } \multicolumn{2}{c|}{} & \multicolumn{6}{c|}{ Prediction } \\
\cline { 3 - 8 } \multicolumn{2}{c|}{} & $\mathrm{A}$ & $\mathrm{B}$ & $\mathrm{C}$ & $\mathrm{D}$ & $\mathrm{E}$ & $\mathrm{F}$ \\
\hline \multirow{3}{*}{} & $\mathrm{A}$ & $0 \%$ & $0 \%$ & $0 \%$ & $0 \%$ & $0 \%$ & $0 \%$ \\
\cline { 2 - 8 } & $\mathrm{B}$ & $0 \%$ & $0 \%$ & $0 \%$ & $0 \%$ & $0 \%$ & $0 \%$ \\
\cline { 2 - 8 } & $\mathrm{C}$ & $0 \%$ & $0 \%$ & $0 \%$ & $0 \%$ & $0 \%$ & $0 \%$ \\
\cline { 2 - 8 } & $\mathrm{D}$ & $0 \%$ & $0 \%$ & $0 \%$ & $0 \%$ & $0 \%$ & $0 \%$ \\
\cline { 2 - 8 } & $\mathrm{E}$ & $0 \%$ & $0 \%$ & $0 \%$ & $0 \%$ & $82.8 \%$ & $.340 \%$ \\
\cline { 2 - 8 } & $\mathrm{F}$ & $0 \%$ & $0 \%$ & $0 \%$ & $0 \%$ & $8.31 \%$ & $99.2 \%$ \\
\hline
\end{tabular}

Table B.5: $\leq 7 \%$ and slope $>25^{\circ}$.

\begin{tabular}{|c|c|c|c|c|c|c|c|}
\hline & & \multicolumn{6}{|c|}{ Prediction } \\
\hline & & $\mathrm{A}$ & B & $\mathrm{C}$ & D & $\mathrm{E}$ & $\mathrm{F}$ \\
\hline \multirow{6}{*}{ 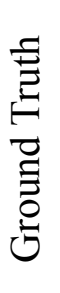 } & A & $0 \%$ & $0 \%$ & $0 \%$ & $0 \%$ & $0 \%$ & $0 \%$ \\
\hline & $\mathrm{B}$ & $0 \%$ & $0 \%$ & $0 \%$ & $0 \%$ & $0 \%$ & $0 \%$ \\
\hline & $\mathrm{C}$ & $0 \%$ & $0 \%$ & $0 \%$ & $0 \%$ & $0 \%$ & $0 \%$ \\
\hline & $\mathrm{D}$ & $0 \%$ & $0 \%$ & $0 \%$ & $0 \%$ & $0 \%$ & $0 \%$ \\
\hline & $\mathrm{E}$ & $0 \%$ & $0 \%$ & $0 \%$ & $0 \%$ & $0 \%$ & $0 \%$ \\
\hline & $\bar{F}$ & $0 \%$ & $0 \%$ & $0 \%$ & $0 \%$ & $0 \%$ & $100 \%$ \\
\hline
\end{tabular}

Table B.6: $\leq 7 \%$ and $10^{\circ}<$ slope $\leq 15^{\circ}$.

\begin{tabular}{|c|c|c|c|c|c|c|c|}
\hline & & \multicolumn{6}{|c|}{ Prediction } \\
\hline & & $\mathrm{A}$ & B & $\mathrm{C}$ & $\mathrm{D}$ & $\mathrm{E}$ & $\mathrm{F}$ \\
\hline \multirow{6}{*}{ 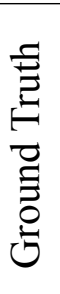 } & $\mathrm{A}$ & $84.0 \%$ & $0 \%$ & $7.50 \%$ & $0 \%$ & $0 \%$ & $.0872 \%$ \\
\hline & B & $0 \%$ & $0 \%$ & $0 \%$ & $0 \%$ & $0 \%$ & $0 \%$ \\
\hline & $\mathrm{C}$ & $10.5 \%$ & $0 \%$ & $81.3 \%$ & $0 \%$ & $0 \%$ & $.636 \%$ \\
\hline & $\mathrm{D}$ & $0 \%$ & $0 \%$ & $0 \%$ & $0 \%$ & $0 \%$ & $0 \%$ \\
\hline & $\mathrm{E}$ & $0 \%$ & $0 \%$ & $0 \%$ & $0 \%$ & $0 \%$ & $0 \%$ \\
\hline & $\mathrm{F}$ & $2.44 \%$ & $0 \%$ & $4.96 \%$ & $0 \%$ & $0 \%$ & $99.2 \%$ \\
\hline
\end{tabular}

Table B.7: $7 \%<$ CFA $\leq 15 \%$ and slope $\leq 10^{\circ}$.

\begin{tabular}{|c|c|c|c|c|c|c|c|}
\cline { 3 - 8 } \multicolumn{2}{c|}{} & \multicolumn{6}{c|}{ Prediction } \\
\cline { 3 - 8 } \multicolumn{2}{c|}{} & $\mathrm{A}$ & $\mathrm{B}$ & $\mathrm{C}$ & $\mathrm{D}$ & $\mathrm{E}$ & $\mathrm{F}$ \\
\hline \multirow{3}{*}{ Truth } & $\mathrm{A}$ & $0 \%$ & $0 \%$ & $0 \%$ & $0 \%$ & $0 \%$ & $0 \%$ \\
\cline { 2 - 8 } & $\mathrm{B}$ & $0 \%$ & $84.0 \%$ & $7.50 \%$ & $0 \%$ & $0 \%$ & $.200 \%$ \\
\cline { 2 - 8 } & $\mathrm{C}$ & $0 \%$ & $10.5 \%$ & $81.3 \%$ & $0.200 \%$ & $0 \%$ & $1.20 \%$ \\
\cline { 2 - 8 } & $\mathrm{D}$ & $0 \%$ & $.300 \%$ & $.600 \%$ & $99.8 \%$ & $0 \%$ & $.200 \%$ \\
\cline { 2 - 8 } & $\mathrm{E}$ & $0 \%$ & $0 \%$ & $0 \%$ & $0 \%$ & $0 \%$ & $0 \%$ \\
\cline { 2 - 8 } & $\mathrm{F}$ & $0 \%$ & $5.20 \%$ & $10.6 \%$ & $0 \%$ & $0 \%$ & $98.4 \%$ \\
\hline
\end{tabular}


Table B.8: $7 \%<\mathrm{CFA} \leq 15 \%$ and $10^{\circ}<$ slope $\leq 15^{\circ}$.

\begin{tabular}{|c|c|c|c|c|c|c|c|}
\cline { 3 - 8 } \multicolumn{2}{c|}{} & \multicolumn{6}{c|}{ Prediction } \\
\cline { 3 - 8 } \multicolumn{2}{c|}{} & $\mathrm{A}$ & $\mathrm{B}$ & $\mathrm{C}$ & $\mathrm{D}$ & $\mathrm{E}$ & $\mathrm{F}$ \\
\hline \multirow{3}{*}{} & $\mathrm{A}$ & $0 \%$ & $0 \%$ & $0 \%$ & $0 \%$ & $0 \%$ & $0 \%$ \\
\cline { 2 - 8 } & $\mathrm{B}$ & $0 \%$ & $84.0 \%$ & $7.50 \%$ & $0 \%$ & $0 \%$ & $.0872 \%$ \\
\cline { 2 - 8 } & $\mathrm{C}$ & $0 \%$ & $10.5 \%$ & $81.3 \%$ & $0 \%$ & $0 \%$ & $.636 \%$ \\
\cline { 2 - 8 } & $\mathrm{D}$ & $0 \%$ & $0 \%$ & $0 \%$ & $0 \%$ & $0 \%$ & $0 \%$ \\
\cline { 2 - 8 } & $\mathrm{E}$ & $0 \%$ & $0 \%$ & $0 \%$ & $0 \%$ & $0 \%$ & $0 \%$ \\
\cline { 2 - 8 } & $\mathrm{F}$ & $0 \%$ & $2.44 \%$ & $4.96 \%$ & $0 \%$ & $0 \%$ & $99.2 \%$ \\
\hline
\end{tabular}

Table B.9: $7 \%<\mathrm{CFA} \leq 15 \%$ and $15^{\circ}<$ slope $\leq 20^{\circ}$.

\begin{tabular}{|c|c|c|c|c|c|c|c|}
\cline { 3 - 8 } \multicolumn{2}{c|}{} & \multicolumn{6}{c|}{ Prediction } \\
\cline { 3 - 8 } \multicolumn{2}{c|}{} & $\mathrm{A}$ & $\mathrm{B}$ & $\mathrm{C}$ & $\mathrm{D}$ & $\mathrm{E}$ & $\mathrm{F}$ \\
\hline \multirow{3}{*}{} & $\mathrm{A}$ & $0 \%$ & $0 \%$ & $0 \%$ & $0 \%$ & $0 \%$ & $0 \%$ \\
\cline { 2 - 8 } & $\mathrm{B}$ & $0 \%$ & $84.0 \%$ & $7.50 \%$ & $0 \%$ & $0 \%$ & $.0872 \%$ \\
\cline { 2 - 8 } & $\mathrm{C}$ & $0 \%$ & $10.5 \%$ & $81.3 \%$ & $0 \%$ & $0 \%$ & $.636 \%$ \\
\cline { 2 - 8 } & $\mathrm{D}$ & $0 \%$ & $0 \%$ & $0 \%$ & $0 \%$ & $0 \%$ & $0 \%$ \\
\cline { 2 - 8 } & $\mathrm{E}$ & $0 \%$ & $0 \%$ & $0 \%$ & $0 \%$ & $0 \%$ & $0 \%$ \\
\cline { 2 - 8 } & $\mathrm{F}$ & $0 \%$ & $2.44 \%$ & $4.96 \%$ & $0 \%$ & $0 \%$ & $99.2 \%$ \\
\hline
\end{tabular}

Table B.10: $7 \%<\mathrm{CFA} \leq 15 \%$ and $20^{\circ}<$ slope $\leq 25^{\circ}$.

\begin{tabular}{|c|c|c|c|c|c|c|c|}
\cline { 3 - 7 } \multicolumn{2}{c|}{} & \multicolumn{6}{c|}{ Prediction } \\
\cline { 3 - 8 } \multicolumn{2}{c|}{} & $\mathrm{A}$ & $\mathrm{B}$ & $\mathrm{C}$ & $\mathrm{D}$ & $\mathrm{E}$ & $\mathrm{F}$ \\
\hline \multirow{3}{*}{} & $\mathrm{A}$ & $0 \%$ & $0 \%$ & $0 \%$ & $0 \%$ & $0 \%$ & $0 \%$ \\
\cline { 2 - 8 } & $\mathrm{B}$ & $0 \%$ & $0 \%$ & $0 \%$ & $0 \%$ & $0 \%$ & $0 \%$ \\
\cline { 2 - 8 } & $\mathrm{C}$ & $0 \%$ & $0 \%$ & $0 \%$ & $0 \%$ & $0 \%$ & $0 \%$ \\
\cline { 2 - 8 } & $\mathrm{D}$ & $0 \%$ & $0 \%$ & $0 \%$ & $0 \%$ & $0 \%$ & $0 \%$ \\
\cline { 2 - 8 } & $\mathrm{E}$ & $0 \%$ & $0 \%$ & $0 \%$ & $0 \%$ & $82.8 \%$ & $.340 \%$ \\
\cline { 2 - 8 } & $\mathrm{F}$ & $0 \%$ & $0 \%$ & $0 \%$ & $0 \%$ & $8.31 \%$ & $99.2 \%$ \\
\hline
\end{tabular}

Table B.11: CFA $>15 \%$ and any slope.

\begin{tabular}{|c|c|c|c|c|c|c|c|}
\cline { 3 - 7 } \multicolumn{2}{c|}{} & \multicolumn{6}{c|}{ Prediction } \\
\cline { 3 - 8 } \multicolumn{2}{c|}{} & $\mathrm{A}$ & $\mathrm{B}$ & $\mathrm{C}$ & $\mathrm{D}$ & $\mathrm{E}$ & $\mathrm{F}$ \\
\hline \multirow{3}{*}{} & $\mathrm{A}$ & $0 \%$ & $0 \%$ & $0 \%$ & $0 \%$ & $0 \%$ & $0 \%$ \\
\cline { 2 - 8 } & $\mathrm{B}$ & $0 \%$ & $0 \%$ & $0 \%$ & $0 \%$ & $0 \%$ & $0 \%$ \\
\cline { 2 - 8 } & $\mathrm{C}$ & $0 \%$ & $0 \%$ & $0 \%$ & $0 \%$ & $0 \%$ & $0 \%$ \\
\cline { 2 - 8 } & $\mathrm{D}$ & $0 \%$ & $0 \%$ & $0 \%$ & $0 \%$ & $0 \%$ & $0 \%$ \\
\cline { 2 - 8 } & $\mathrm{E}$ & $0 \%$ & $0 \%$ & $0 \%$ & $0 \%$ & $0 \%$ & $0 \%$ \\
\cline { 2 - 8 } & $\mathrm{F}$ & $0 \%$ & $0 \%$ & $0 \%$ & $0 \%$ & $0 \%$ & $100 \%$ \\
\hline
\end{tabular}

